\author{
Universidade de São Paulo \\ Instituto de Astronomia, Geofísica e Ciências Atmosféricas
}

Departamento de Astronomia

Felipe Andrade Oliveira

\title{
Cosmologias Aceleradas com Criação de Matéria: Teoria e Testes Observacionais
}

São Paulo 

Felipe Andrade Oliveira

\section{Cosmologias Aceleradas com Criação de}

\section{Matéria: Teoria e Testes Observacionais}

Tese apresentada ao Departamento de Astronomia do Instituto de Astronomia, Geofísica e Ciências Atmosféricas da Universidade de São Paulo como requisito parcial para a obtenção do título de Doutor em Ciências.

Versão Corrigida. O original encontra-se disponível na Unidade.

Área de Concentração: Astronomia Orientador: Prof. Dr. José Ademir Sales de Lima

São Paulo 

À minha Família e aos meus Amigos. 



\section{Agradecimentos}

Ao meu orientador José Ademir de Sales de Lima, pelo apoio e sugestão do tema.

Aos colaboradores José Fernando de Jesus, Spyros Basilakos e Francisco Ernandes Costa, pela amizade, pelas frutíferas discussões e pelas colaborações em artigos.

Ao relator Jorge Ernesto Horvath, pelos conselhos e agradáveis discussões.

Aos outros professores membros da banca de defesa, Wilson Zanchin, Marcos Lima e Gastão Bierrenbach, pelas críticas construtivas ao trabalho e pelo auxílio na melhora do presente texto.

Aos amigos Vinicius Consolini Busti e José Fernando de Jesus, pela leitura cuidadosa desta tese, pelo trabalho ao longo do doutorado e pelo prazer da convivência.

Ao grupo de cosmologia, Leila Graef, Rodrigo Holanda, Silvio Fiorentin, Miguel de Campos, Rose Santos, Saulo Pereira, Eder Leonardo Perico, Iuri Baranov, Antônio Cândido Guimarães, Joao Maria da Silva, João Vital da Cunha, Giorgi Zilioti, Diego Oliveira, David Alejandro Tamayo, pela colaboração e amizade.

A toda a minha família, em especial aos meus pais Maria Silvana Andrade da Silva, Estevan Antônio de Oliveira Neto, ao meu irmão Leandro Andrade Oliveira e a toda minha família, por acreditarem no poder da educação e pelo apoio incondicional, mesmo nunca tendo compreendido exatamente o que eu estudo.

Aos queridos amigos dos tempos de CEFET e agregados Nádia, Marcus Vinícius, Da-

nilo, Virgínia, Vinicius, Vanessa, Grazi, Carlos Felipe, Andrezza, Thais Ogata, professor Marcelo Oliveira entre muitos outros professores e amigos, pelo apoio e pela amizade que resiste ao tempo.

Aos meus amigos dos tempos de IFUSP, André Modesto, José Amorim, Clodoaldo José, Thiago (Castor) Pereira, Wellgton (Cabloco) Ferreira e a todos outros que participaram e 
participam da minha vida.

Aos meus muitos e muito queridos amigos do IAG, que foram parte importante da época mais feliz que vivi na USP, entre eles, Thiago (Zé Colmeia) Almeida, Gustavo Rocha, Alan Jorge, Thiago (Gerson) Junqueira, Alberto Krone-Martins, Rodrigo Souza, Rafael (Tamas) Kimura, Douglas Barros, Carlos Braga, Thiago Ricci, Juliana Motter, Pedro Beaklini, Nathália Cibirka, Patrícia Novais, Fernanda Urrutia, Reinaldo Santos, Rafael Santucci, Luciene Coelho, Alesandro Moisés, Felipe Santos, Juan Pineda, Bruno Quint, Luis Kadowaki, Walter Santos, Rodrigo Vieira, Luiz Felippe Santiago, Greg Kowal, Vinícius Placco, Oscar Cavichia e a todos os outros com quem dividi conversas, cafés e reflexões.

À minha querida, amada, paciente esposa e amiga Aline Cristina Nunes de Almeida, pela companhia, apoio e vida cheia de alegria. Também aos meus sogros Maria Lúcia, Claudiomar, aos meus sobrinhos e cunhados pela linda extensão de família que ganhei.

Aos Professores Eduardo Cypriano, Laerte Sodré, Cláudia Mendes de Oliveira, Gastão Bierrenbach Lima Neto, Amâncio Friaça, Jane Gregorio Hetem, Roy Maartens, Sandra dos Anjos, Ronaldo de Souza, Raul Abramo e Gary Steigman pela generosidade e dedicação na formação dos estudantes e, em particular, na minha. Sinto-me muito lisonjeado pela convivência e aprendizado.

A todos os funcionários e técnicos do IAG, em especial Ulisses, Marco, Luis, Carlos, Marina, Regina, Conceição, Cida, Romilda e Silvana.

Ao CNPq, pelo apoio financeiro, sob o projeto $n^{o}: 143464 / 2011-6$ e à Universidade de São Paulo, pela minha formação e pelos seus programas de assistência estudantil.

Esta tese/dissertação foi escrita em LATEX com a classe IAGTESE, para teses e dissertações do IAG. 


"Tudo, aliás, é a ponta de um mistério, inclusive os fatos. Ou a ausência deles."

Guimarães Rosa

"O fato mais assustador a respeito do universo não é que ele seja hostil, mas que seja indiferente; porém, se pudermos entrar em acordo com essa indiferença e aceitar os desafios da vida dentro dos limites da morte - por mais que o Homem possa fazê-los mutáveis-, nossa existência como espécie pode ter um significado genuíno e satisfatório.

Por mais vasta que seja a escuridão, nós devemos nos fornecer luz própria."

Stanley Kubrick

"A Astronomia é útil porque nos eleva acima de nós mesmos, é útil porque é grande; é útil porque é bela; isso é o que se precisa dizer. É ela que nos mostra o quanto o homem é pequeno no corpo e o quanto é grande no espírito, já que nesta imensidão resplandecente, onde seu corpo não passa de um ponto obscuro, sua inteligência pode abarcar inteira e dela fluir a silenciosa harmonia. Atingimos assim a consciência de nossa força, e isso é uma coisa pela qual jamais pagaríamos caro demais, porque essa consciência nos torna mais fortes." 



\section{Resumo}

Os recentes avanços em cosmologia observacional indicam que o universo esteja passando por uma fase de expansão acelerada. A determinação do mecanismo responsável pela aceleração cósmica constitui um dos problemas mais intrigantes na ciência hoje. Entre os diversos candidatos a mecanismo de aceleração, a explicação mais simples e econômica é assumir a existência de uma constante cosmológica $\Lambda$ associada à energia do vácuo. Contudo, essa interpretação leva a importantes problemas conceituais associados à natureza dessa componente.

Nesta tese, investigamos a dinâmica de diferentes mecanismos de aceleração cósmica, comparando suas previsões com diversos testes observacionais. Em particular, demos ênfase aos cenários baseados na criação de matéria escura fria (CCDM), nos quais a presente aceleração do universo é produzida sem a presença de um fluido exótico, como consequência do processo de produção de partículas de matéria escura gravitacionalmente induzido.

Inicialmente, propusemos um modelo no qual o mecanismo de criação de partículas é capaz de gerar uma cosmologia dinamicamente degenerada com o modelo padrão, $\Lambda$ CDM. Discutimos no chamado modelo de Lima, Jesus \& Oliveira (LJO) a dinâmica cosmológica com criação de matéria escura fria e com pressão. Através de um teste estatístico de $\chi^{2}$, mostramos que o modelo fornece ótimo ajuste aos dados de supernovas tipo Ia (SNe Ia).

Posteriormente, estudamos a evolução de pequenas perturbações de densidade em um fundo homogêneo para modelos tipo CCDM, através do formalismo Neo-Newtoniano. Restringindo-nos ao modelo LJO, comparamos as previsões obtidas nesse contexto com as proveniente do modelo $\Lambda \mathrm{CDM}$. Mostramos que o modelo é capaz de fornecer excelente ajuste aos dados observacionais de medidas da taxa de crescimento linear, para o caso plano e com velocidade efetiva do som $c_{e f f}^{2}=-1$. 
Ainda dentro do cenário CCDM, investigamos uma segunda proposta original, com capacidade de ajuste às observações similar aos modelos $\Lambda$ CDM e LJO com mesmo número de parâmetros livres, porém com dinâmica não degenerada com estes. Derivamos a dinâmica cosmológica do modelo e discutimos a sua viabilidade através da análise estatística de medidas de SNe Ia e do parâmetro de Hubble em diferentes redshifts $H(z)$.

Finalmente, discutimos a dinâmica de um modelo com decaimento do vácuo $(\Lambda(\mathrm{t}) \mathrm{CDM})$ e sua descrição em campos escalares. Assumindo como forma do termo de vácuo uma série de potências truncada do parâmetro de Hubble, derivamos as equações dinâmicas básicas e as previsões cosmológicas do modelo. Mostramos que, quando a transferência de energia entre as componentes dos setor escuro se dá através da criação de partículas, modelos CCDM e $\Lambda(\mathrm{t}) \mathrm{CDM}$ podem compartilhar a mesma dinâmica e termodinâmica, dentro de certas condições. Adicionalmente, mostramos que o modelo é capaz de prover um bom ajuste às medidas de SNe Ia e da chamada razão $\mathrm{CMB} / \mathrm{BAO}$. Obtivemos ainda uma descrição do modelo $\Lambda(t) \mathrm{CDM}$ por um campo escalar, estendendo a validade do modelo para outros espaços-tempos e outras teorias gravitacionais.

Nossos resultados mostram que existem diversas alternativas viáveis ao atual modelo padrão em cosmologia, capazes de contornar os problemas associados à constante cosmológica. A discussão dessas alternativas é essencial para uma compreensão mais profunda acerca da dinâmica, da composição e do destino do universo. 


\section{Abstract}

Recent advances in observational cosmology indicate that the universe is undergoing an accelerating stage of expansion. The determination of the mechanism responsible for the cosmic acceleration is one of the most intriguing problems in science today. Among many candidates for the acceleration mechanism, the simplest and most economical explanation is to assume the existence of a cosmological constant $\Lambda$ associated with the vacuum energy. However, this interpretation leads to important conceptual problems associated with the nature of this component.

In this thesis, we investigate the dynamics of different mechanisms of cosmic acceleration, comparing their predictions through several observational tests. In particular, we emphasize the scenarios based on creation of cold dark matter (CCDM), in which the present acceleration of the universe is produced without the presence of an exotic fluid as a result of the gravitationally induced dark matter production process.

Initially, we have proposed a model in which the particle creation mechanism is able to produce a cosmology dynamically degenerated with respect to the standard model, $\Lambda \mathrm{CDM}$. We discussed the cosmological dynamics for the creation of cold dark matter and dark matter with pressure within the so-called model of Lima, Jesus \& Oliveira (LJO). Through a statistical $\chi^{2}$ test, we showed that the model provides a good fit to the type Ia supernovae (SNe Ia) data.

Subsequently, we studied the evolution of small density perturbations in a homogeneous background for CCDM type models through the Neo-Newtonian formalism. Restricting ourselves to the LJO model, the predictions obtained in this context were compared with those from the $\Lambda \mathrm{CDM}$ model. We showed that the model is able to provide a very good fit to the linear growth rate observational data, for a flat universe and effective speed of 
sound $c_{e f f}^{2}=-1$.

Also within the CCDM scenario, we discussed a second original proposal with similar goodnes-of-fit and same degree of freedom to the models $\Lambda$ CDM and LJO, but with a non-degenerated dynamics. We derived the dynamics of the model and discussed its viability through the statistical analysis of measurements of SNe Ia and Hubble parameter in different redshifts $H(z)$, obtaining results in complete agreement.

Finally, we discussed the dynamics of a model with vacuum decay $(\Lambda(\mathrm{t}) \mathrm{CDM})$ and its description by scalar fields. Assuming the form of the vacuum term as a power series in the Hubble parameter, we derived the basic dynamic equations and the cosmological predictions of the model. We showed that when the energy exchange between the components of the dark sector is through the creation of particles, CCDM models and $\Lambda(\mathrm{t}) \mathrm{CDM}$ can share the same dynamics and thermodynamics under certain conditions. Additionally, we showed that the model is able to provide a good fit to the SNe Ia data and measurements of the $\mathrm{CMB} / \mathrm{BAO}$ ratio. We also derived a description of $\Lambda \mathrm{CDM}$ model by a scalar field, extending the validity of the model for other space-times and other gravitational theories.

Our results show that there are several viable alternatives to the current standard model of cosmology, able to overcome the problems associated with the cosmological constant. The discussion of these alternatives is essential to a deeper understanding of the dynamics, composition and fate of the universe. 


\section{Notação e Convenções}

- Assinatura da métrica: $(+,-,-,-)$.

- Índices gregos variam de 0 a 3 , índices latinos variam de 1 a 3 . Índices repetidos obedecem à convenção de Einstein.

- Derivada parcial: $\frac{\partial \phi}{\partial x^{\alpha}} \equiv \phi,_{\alpha}$.

- Derivada covariante: $A^{\alpha}{ }_{\beta}=A^{\alpha},{ }_{\beta}+\Gamma_{\lambda \beta}^{\alpha} A^{\lambda}$.

- Salvo menção contrária, usaremos um sistema de unidades onde c=1.

- Expressões em outros idiomas serão escritas em itálico. 



\section{Informação Eletrônica}

A maioria das referências bibliográficas utilizadas nessa dissertação pode ser encontrada nas seguintes páginas da WEB:

- http://www.periodicos.capes.gov.br/

- http://adsabs.harvard.eduabstract_service.html

- http://xxx.lanl.gov/

Os dados de Supernovas do Tipo Ia da compilação Union 2.1 estão disponíveis em:

- http://supernova.lbl.gov/Union/ 



\section{Lista de Figuras}

2.1 Comparação entre os mapas do céu em micro-ondas medidos pelos projetos COBE (Smoot et al., 1992), WMAP (Hinshaw et al..,2003) e Planck (Planck Collaboration XVI: Ade, P. A. R. et al., 2013). . . . . . . . . . . . . . 48

2.2 Medidas das anisotropias da CMB, realizadas por Planck Collaboration XVI: Ade, P. A. R. et al. (2013).

2.3 Curva de rotação da galáxia espiral NGC6503. Os pontos representam medidas da velocidade de rotação em função da distância ao centro galáctico. As linhas representam as contribuições para a velocidade rotacional de cada uma das componentes visíveis (disco e gás) e da componente escura (halo) da galáxia. A adição de uma componente de halo permite o ajuste das observações de velocidade, sendo mais importante para grandes distâncias do centro galáctico. Figura retirada de Begeman et al. (1991).

2.4 Imagens do aglomerado em fusão 1E0657-558 (Bullet Cluster) Esquerda:) no visual e Direita:) em exposição de 500 ks em raios-X . Os contornos verdes mostram a função $\kappa$, proporcional à densidade superficial de massa. O contorno mais externo representa $\kappa=0.16$ e os contornos internos foram calculados subtraindo-se $\Delta \kappa=0.07$. A barra branca no canto inferior tem 500 kpc na posição do aglomerado. Figura extraída de Clowe et al. (2006).

2.5 Diagrama de Hubble-Sandage contendo as $42 \mathrm{SNe}$ Ia observadas pelo $\mathrm{Su}$ pernova Cosmology Project (Perlmutter et al., 1999). . . . . . . . . . . . . .

2.6 Diagrama de Hubble-Sandage das observações de supernovas do Tipo Ia contendo as 16 SNe Ia observadas pelo High-z Supernova Search (Riess et al., 1998). 
2.7 Vínculos obtidos sobre os parâmetros livres do modelo $\Lambda$ CDM. As regiões com $68.3 \%$, $95.4 \%$ e $99.7 \%$ de nível de confiança estatística no plano $\Omega_{m}-\Omega_{\Lambda}$ delimitam os vínculos obtidos das análises de SNe Ia (azul), BAO (verde), CMB (laranja) e da combinação dos anteriores (cinza). Os vínculos obtidos considerando-se apenas os erros estatísticos na análise de SN Ia são mostrados do lado esquerdo. Ao lado direito, são mostrados as regiões de confiança obtidas considerando-se os erros estatísticos e sistemáticos em SN Ia. Figura retirada de (Suzuki et al., 2012).

5.1 Esquerda:) Plano $\Omega_{m}-\alpha$ para o modelo LJO com curvatura, obtido através da análise de 580 dados de SN Ia do catálogo Union 2.1 (Suzuki et al., 2012). As regiões delimitam os níveis de confiança estatística de $68.3 \%, 95.4 \%$ e $99.7 \%$ e a linha preta passa pelos pontos no plano em que a condição de planura é satisfeita. Direita:) Níveis de confiança no plano $\Omega_{m}-\alpha$ para $68.3 \%, 95.4 \%$ e $99.7 \%$ de confiança estatística. Note que neste plano, o caso plano equivale a $\Omega_{m}=1$.

5.2 Vínculos obtidos sobre o parâmetro $\alpha$ no caso plano.

5.3 Vínculos sobre os parâmetros livres do modelo LJO plano com criação de matéria com pressão em $1 \sigma, 2 \sigma$ e $3 \sigma$ de nível de confiança. Os limites foram estimados considerando a amostra de dados de SN Ia Union 2.1. Os valores de melhor ajuste encontrados $\alpha=0.723_{-0.319}^{+0.111}$ e $\varepsilon=0.001_{-0.422}^{+0.333}$ para $1 \sigma \mathrm{com}$ $\chi_{\text {min }}^{2}=562.2$ e valor reduzido de $\chi_{\nu}^{2}=0.97 \ldots \ldots \ldots \ldots$

6.1 Contraste de densidade $\delta$ em função de $a$ para o caso $c_{e f f}^{2}=0$. Para o modelo LJO, este caso é equivalente a $c_{e f f}^{2}=c_{s}^{2}$ pois a velocidade do som adiabática é zero. Comparamos também o caso $\Lambda$ CDM plano, com $\Omega_{m}=1-\Omega_{\Lambda}=0.3$

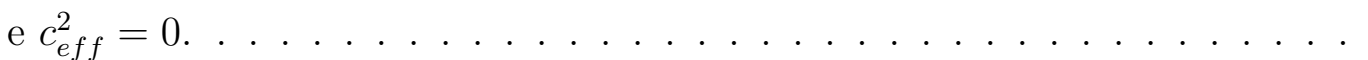

6.2 Contraste de densidade $\delta$ em função de $a$ para o caso $c_{e f f}^{2}=\omega$. Por comparação, mostramos a solução para o caso $\Lambda$ CDM plano, com a identificação $\Omega_{m}=1-\alpha=0.3$ e $c_{e f f}^{2}=0$. As curvas obtidas foram tomadas no limite de grandes escalas $(k \rightarrow 0)$ 
6.3 Contraste de densidade $\delta$ em função de $a$ para o caso em que $c_{\text {eff }}^{2}$ é um parâmetro livre. Adicionalmente, mostramos o caso $\Lambda \mathrm{CDM}$ plano, com $\Omega_{m}=0.3$ e $c_{\text {eff }}^{2}=0$. As curvas mostradas foram obtidas para o limite de grandes escalas $(k \rightarrow 0)$ e $\alpha=0.7 \ldots \ldots \ldots \ldots$

6.4 Esquerda:) Plano $c_{e f f}^{2}-\alpha$ para o modelo LJO plano considerando-se dados de $f(z)$. As regiões delimitam $68.3 \%, 95.4 \%$ e $99.7 \%$ de confiança estatística. Direita:) Medidas de $f(z)$ e curvas teóricas obtidas com os valores de melhor ajuste do modelo LJO (linha sólida vermelha) e do modelo $\Lambda$ CDM (linha tracejada preta) (Jesus et al., 2011). . . . . . . . . . . . . . 101

7.1 Soluções numéricas de parâmetro de Hubble normalizado $H / H_{0}$ em função do redshift z e suas dependências com os parâmetros livres $\alpha$ e $\Omega_{k}$. Esquerda: Evolução de $H(z) / H_{0} \operatorname{com} \alpha=0.7$ e $\Omega_{k}=0$ (em vermelho), $\Omega_{k}=0.6$ (em azul) e $\Omega_{k}=-0.5$ (em verde). Direita: Evolução de $H(z) / H_{0}$ com $\Omega_{k}=0$ e $\alpha=0$ (em azul), $\alpha=0.7$ (em vermelho) e $\alpha=0.9$ (em verde). 109

7.2 Plano $\alpha-\Omega_{k}$ para o modelo CCDM. Esquerda: Vínculos obtidos através da análise de 580 dados de SN Ia do catálogo Union 2.1 (Suzuki et al., 2012) e de 28 dados de $H(z)$ compilados em Farooq e Ratra (2013) . Os contornos delimitam os níveis de confiança estatística de 68.3\%, 95.4\% e 99.7 \%. Direita: Vínculos obtidos considerando-se a combinação de dados de SN Ia, $H(z)$ e um prior sobre o parâmetro de curvatura $\Omega_{k}$ (Planck Collaboration XVI: Ade, P. A. R. et al., 2013) para 68.3\%, 95.4\% e 99.7\% de confiança estatística.

7.3 Soluções numéricas de $H$ em função do redshift $z$ nos modelos CCDM e $\Lambda \mathrm{CDM}$ e suas dependências com os parâmetros livres $\alpha$ e $\Omega_{k}$. Cada região colorida corresponde a uma solução numérica para um conjunto de valores $\left(\alpha, \Omega_{k}\right)$ e à variação de $H_{0}$ em um $1 \sigma$ de incerteza $\left(H_{0}=72.0 \pm\right.$ $\left.3.0 \mathrm{~km} \mathrm{~s}^{-1} \mathrm{Mpc}^{-1}\right)$.

8.1 Resultados da análise estatística. Os vínculos de SNe Ia e da razão CMB/BAO são mostrados nos regiões em azul e laranja, respectivamente. As linhas tracejadas delimitam os vínculos obtidos da combinação dos dados observacionais para $68.3 \%, 95.4 \%$ e $99.73 \%$ de nível de confiança. 
8.2 Evolução do potencial do campo escalar em unidades da densidade de energia crítica hoje $U(\phi) \equiv V(\phi) / \rho_{c, 0}$. As curvas pontilhada, sólida e tracejada correspondem respectivamente aos valores do limite inferior, melhor ajuste e limite superior dos parâmetros $\Omega_{m}$ e $\beta$ em $1 \sigma$ de nível de confiança (Andrade-Oliveira, F. et al., 2014). . . . . . . . . . . . . . . . . . 


\section{Lista de Tabelas}

2.1 Valores de melhor ajuste para os parâmetros do modelo $\Lambda$ CDM e variação em $68.7 \%$ de nível de confianca estatística. Limites obtidos por Betoule

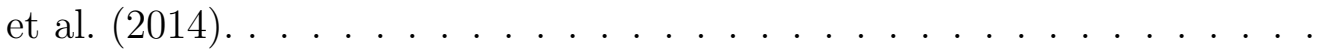

3.1 Valores de melhor ajuste e limites em $1 \sigma$ de confiança estatística dos principais parâmetros do modelo $\Lambda$ CDM obtidos pelo satélite Planck (i) através das análises dos dados do espectro de potências de temperatura da CMB (colunas 2 e 3), (ii) da combinação entre os dados de temperatura e efeito de lentes gravitacionais (colunas 4 e 5) e (iii) da combinação entre os dados de temperatura com os dados de polarização da CMB em baixos multipolos do satélite WMAP (colunas 6 e 7). Tabela extraída de (Planck Collaboration XVI: Ade, P. A. R. et al., 2013). . . . . . . . . . . . . . . . . . . .

5.1 Comparação entre os modelos $\Lambda$ CDM e LJO . . . . . . . . . . . . .

6.1 Dados da taxa de crescimento $f(z) \ldots \ldots \ldots$. . . . . . . . . . . . . . . .

7.1 Dados de $H(z)$ em função do redshift z e suas incertezas. Na coluna mais à direita da tabela acima, são apresentadas as referências de cada um dos dados de $H(z)$, conforme a compilação realizada por Farooq e Ratra (2013). 117

7.2 Resultados da análise estatística para os parâmetros livres do modelo em diferentes combinações de dados. Os limites apresentados correspondem a $68.3 \%$ de nível de confiança estatística. . . . . . . . . . . . . . 118

8.1 Resultados da análise estatística combinada. . . . . . . . . . . . 134 
A.1 Valor do $\Delta \chi^{2}$ em função do nível de confiança e do número de parâmetros livres M. Tabela extraída de (Press et al., 1992). . . . . . . . . . . 170

A.2 Critérios quantitativos para a seleção de modelos via BIC (Kass e Raftery, 1995) . . . . . . . . . . . . . . . . . . . . . . 171 


\section{Sumário}

1. Introdução . . . . . . . . . . . . . . . . . . . . . . . . . . 29

2. Cosmologia Física . . . . . . . . . . . . . . . . . . . . . 35

2.1 Introdução . . . . . . . . . . . . . . . . . . . . . 35

2.2 Teoria da Relatividade Geral . . . . . . . . . . . . . . . . . 35

2.3 Métrica de Friedmann-Lemaître-Robertson-Walker . . . . . . . . . . . . . . 39

2.4 Dinâmica Cosmológica . . . . . . . . . . . . . . . . . . . 39

2.5 Parâmetros Cosmológicos . . . . . . . . . . . . . . . . . 40

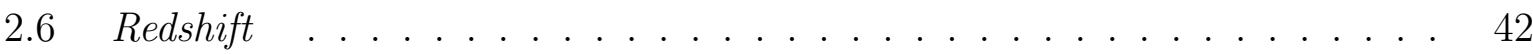

2.7 Modelos de Big Bang . . . . . . . . . . . . . . . . . . . . . . . . 43

2.7.1 A Expansão do Universo . . . . . . . . . . . . . . . . . . . . . . 43

2.7.2 Nucleossíntese Primordial . . . . . . . . . . . . 44

2.7.3 Radiação Cósmica de Fundo . . . . . . . . . . . . . . . . . 45

2.7.4 Distâncias Cosmológicas . . . . . . . . . . . . . . 48

2.7 .5 Idade do Universo . . . . . . . . . . . . . . . . . . . 51

2.7 .6 Matéria Escura . . . . . . . . . . . . . . 52

2.7 .7 Aceleração Cósmica . . . . . . . . . . . . . . . . 56

2.7.8 A Consolidação de um Novo Paradigma . . . . . . . . . . . . 58

3. Modelos Acelerados . . . . . . . . . . . . . . . . . . . . 61

3.1 A Constante Cosmológica $\Lambda \ldots \ldots \ldots \ldots$. . . . . . . . . . .

3.2 Matéria X, Phantom \& CPL . . . . . . . . . . . . 65

3.3 Quintessência \& Campos Escalares . . . . . . . . . . . . . . . 66

3.4 Gás de Chaplygin . . . . . . . . . . . . . . . . . . . . . 68 
3.5 Modelos com Decaimento do Vácuo

4. Cosmologias com Criação de Matéria . . . . . . . . . . . . . . . . 73

4.1 Introdução . . . . . . . . . . . . . . . . . . . . . . . . 73

4.2 Termodinâmica da Criação de Partículas . . . . . . . . . . . . . 75

4.3 Dinâmica Cósmica e Criação de Matéria Escura . . . . . . . . . . . . . . 76

5. Criação de Matéria: Modelo de Lima, Jesus \& Oliveira . . . . . . . . . . . . . . 79

5.1 Introdução . . . . . . . . . . . . . . . . . . . . . . . . . . 79

5.2 Modelo de Lima, Jesus \& Oliveira (LJO) . . . . . . . . . . . . . . . . 80

5.3 Criação de Matéria Escura com Pressão no Cenário LJO . . . . . . . . . . 83

5.4 Vínculos Observacionais no Modelo LJO . . . . . . . . . . . . . 84

5.5 Discussões . . . . . . . . . . . . . . . . . . . 87

6. Perturbações Lineares no Modelo de Lima, Jesus \& Oliveira . . . . . . . . . . . 89

6.1 Introdução . . . . . . . . . . . . . . . . . . . . . . 89

6.2 Formulação Neo-Newtoniana para Cosmologias com Criação de Matéria . . 89

6.2.1 Modelo CCDM na Abordagem Neo-Newtoniana . . . . . . . . . . . 90

6.3 Perturbações de Densidade na Formulação Neo-Newtoniana . . . . . . . . . 91

6.4 Perturbações de Densidade no Modelo LJO . . . . . . . . . . . . . . . . . 95

6.5 Análise Observacional e a Taxa de Crescimento das Perturbações . . . . . 98

6.6 Discussões . . . . . . . . . . . . . . . . . . . . . . . 102

7. Um Novo Modelo CCDM com Curvatura Espacial . . . . . . . . . . . . . . 105

7.1 Introdução . . . . . . . . . . . . . . . . . . . 105

7.2 Dinâmica Cósmica . . . . . . . . . . . . . . . . 106

7.3 Vínculos Observacionais . . . . . . . . . . . . . . . . . 108

7.3.1 Limites obtidos através do Parâmetro de Hubble . . . . . . . . . . . 109

7.3.2 Limites obtidos através de Supernovas do Tipo Ia . . . . . . . . . . 111

7.3.3 Limites Combinados . . . . . . . . . . . . . . . . 112

7.4 Discussões . . . . . . . . . . . . . . . . . . . . . . . . . . . . 114

8. Modelo com Decaimento do Vácuo e Descrição por Campos Escalares . . . . . . 119

8.1 Introdução . . . . . . . . . . . . . . . . . . . . . . . . . . 119 
8.2 O Modelo $\Lambda(t) \quad \ldots \ldots \ldots \ldots \ldots \ldots \ldots$

8.3 Criação de Matéria ou Variação de Massa? . . . . . . . . . . . . . . . . 123

8.4 Segunda Lei da Termodinâmica Generalizada . . . . . . . . . . . . . . 127

8.5 Análise Observacional . . . . . . . . . . . . . . . . . . . . . . 130

8.5.1 Vínculos de Supernovas Tipo Ia . . . . . . . . . . . . . . . . . 131

8.5.2 Vínculos da razão CMB/BAO . . . . . . . . . . . . . . 131

8.5.3 Análise Conjunta . . . . . . . . . . . . . . . . . . . . 132

8.6 Descrição de Campo Escalar . . . . . . . . . . . . . . . . . . . . . . 134

8.7 Discussões . . . . . . . . . . . . . . . . . . . . . . . . . . . . . . . . 137

9. Conclusões . . . . . . . . . . . . . . . . . . . . . . . . . . . . . 139

Referências ............................ . . . 143

Apêndice 167

A. Análise Estatística . . . . . . . . . . . . . . . . . . . . . . . . . 169 

Capítulo 1

\section{Introdução}

A Cosmologia é a área da Ciência que estuda o universo em sua origem, evolução, composição, formação de estruturas e características em grandes escalas. Embora questões cosmológicas tenham sempre permeado a cultura humana, a Cosmologia moderna nasceu logo após o estabelecimento da Teoria da Relatividade Geral (TRG). A TRG é uma teoria física sobre o espaço, o tempo e a gravitação, sendo atualmente considerada o principal arcabouço teórico para estudar o universo em grandes escalas.

Nosso conhecimento em Cosmologia é expresso através dos chamados modelos de universo ou modelos cosmológicos, criados a partir de leis e hipóteses básicas acerca do universo. Um dos princípios mais utilizados na formulação desses modelos é o Princípio Cosmológico, i.e., a hipótese de que o universo não possua observadores privilegiados e, como consequência, seja homogêneo e isotrópico em grandes escalas.

O primeiro modelo cosmológico moderno, criado por A. Einstein (1917), descreve um universo estático e constituído apenas por matéria sem pressão homogeneamente distribuída, conforme a crença geral na época. Para esse fim, Einstein adicionou à sua teoria gravitacional a constante $\Lambda$, conhecida como constante cosmológica, capaz de contrabalancear a atração gravitacional da matéria.

Outros modelos logo foram propostos. Ainda em 1917, W. de Sitter criou um modelo assumindo a hipótese de que a quantidade total de matéria do universo pudesse ser desprezível. O chamado modelo de de Sitter (1917) é obtido das soluções das equações de campo de Einstein com o universo composto apenas pela constante cosmológica.

Na primeira metade da década de 1920, A. A. Friedmann percebeu que as equações de campo admitiam soluções dinâmicas para os casos $\Lambda=0$ e $\Lambda \neq 0$. Nasceram então os cha-

mados modelos expansionistas de Friedmann com curvatura espacial positiva (Friedmann, 
1922) e com curvatura negativa (Friedmann, 1924). Poucos anos depois, G. Lemaître (1927) mostrou que o equilíbrio entre a atração gravitacional e a repulsão cosmológica no modelo de Einstein era instável. Dessa forma, uma mínima diferença entre os termos atrativos e repulsivos faria com que o universo ou entrasse em colapso ou se expandisse eternamente.

A hipótese de um universo dinâmico ganhou ainda mais força com as observações de Lemaître (1927) e E. Hubble (1929), quando a expansão do universo recebeu suporte observacional. Ambos mostraram que os espectros de luz de galáxias distantes apresentavam um desvio sistemático para as regiões mais vermelhas (redshift), proporcionalmente à distância em que as galáxias se encontravam. Essa relação linear, conhecida como lei de Hubble, é prevista pelos modelos de Friedmann, nos quais o afastamento entre as galáxias é compreendido como a expansão do próprio universo.

Com isso, Einstein abandonou definitivamente a ideia de universo estático. Em 1932, em colaboração com de Sitter, foi proposto o modelo de universo com constante cosmológica nula $(\Lambda=0)$, seção espacial plana e constituído apenas por matéria não relativística, batizado como modelo de Einstein-de Sitter (EdS) (Einstein e de Sitter, 1932). O conjunto de todos esses modelos expansionistas estão na base da cosmologia moderna.

Lemaitre (1934), baseado no fato de que o universo está se expandindo, propôs a ideia de um átomo primordial, extrapolando as equações expansionistas para um momento no passado em que a matéria deveria estar concentrada em único ponto. Esse instante inicial do universo foi posteriormente batizado de Big Bang. As soluções expansionistas foram então classificadas do ponto de vista de simetrias por H. Robertson (1936) e A. Walker (1936), sendo atualmente conhecidas em sua forma geral de métrica de FriedmannLemaître-Robertson-Walker (FLRW).

Tendo como base os modelos expansionistas, G. Gamow e colaboradores investigaram a possibilidade da síntese dos elementos químicos no universo primordial (Gamow, 1946; Alpher et al., 1948). A nucleossíntese primordial é, portanto, a teoria que explica a formação dos elementos mais leves no universo, como o deutério (D), hélio $\left({ }^{3} H e\right.$ e $\left.4^{H} e\right)$ e lítio $\left({ }^{7} \mathrm{Li}\right)$. Além disso, durante o seu desenvolvimento, Gamow propôs que o universo atualmente deveria ser permeado por um campo de radiação homogêneo, isotrópico e com o espectro característico de um corpo negro de temperatura $T \lesssim 10 K$ (Alpher et al., 1948). A radiação cósmica de fundo (CMB - Cosmic Microwave Background) foi ocasional- 
mente observada por Penzias e Wilson (1965), evidenciando que o universo esteve em passado extremamente quente e denso. Em 1992, o satélite COBE pôde determinar que o espectro medido da CMB se ajustava fantasticamente bem a uma curva de corpo negro com temperatura em torno de $2.73 K$ (Smoot et al., 1992). As observações da expansão do universo, as medidas das abundâncias relativas dos elementos leves previstas pela nucleossíntese primordial, a existência da radiação cósmica de fundo altamente homogênea e isotrópica e a TRG dão forte sustentação ao modelo de Big Bang.

Até os anos 1990, o modelo de Einstein-de Sitter era considerado como modelo padrão do universo, embora algumas observações não fossem satisfatoriamente explicadas. Medidas da idade de objetos velhos exigiam que a constante de Hubble tivesse valor $H_{0} \leq$ $51 \mathrm{~km} \mathrm{~s}^{-1} M p c^{-1}$, com o parâmetro densidade de matéria $\Omega_{m}=1$ ou $H_{0} \leq 63 \mathrm{~km} \mathrm{~s}^{-1} \mathrm{Mpc}^{-1}$, se $\Omega_{m}=0.3$ (Vandenberg et al., 1996). Por outro lado, a planura medida na CMB em um modelo de Einstein-de Sitter sugeria $\Omega_{m}=1$ e as medidas da constante de Hubble na época indicavam $H_{0}=80 \pm 17 \mathrm{~km} \mathrm{~s}^{-1} \mathrm{Mpc-1}$ (Freedman et al., 1994).

Em 1998, através de observações de Supernovas do Tipo Ia, dois grupos concluíram, de forma independente que o universo está passando por uma fase de expansão acelerada (Riess et al., 1998; Perlmutter et al., 1999). Dessas observações, inferiu-se que o universo hoje é formado por aproximadamente $70 \%$ de uma componente escura e com pressão negativa, genericamente chamada de energia escura. A existência desse termo é compatível com observações das anisotropias da radiacão cósmica de fundo (Planck Collaboration XVI: Ade, P. A. R. et al., 2013), medidas de estruturas em grandes escala (Tegmark et al., 2004; Cole et al., 2005; Percival et al., 2010), idade de objetos velhos em grandes redshifts (Krauss, 1997; Alcaniz e Lima, 1999, 2001; Alcaniz et al., 2003; Cunha e Santos, 2004; Lima et al., 2009), observações em raios-X de aglomerados de galáxias (Allen et al., 2002; Lima et al., 2003a; Allen et al., 2008), tamanho angular de fontes de rádio compactas e de rádio galáxias (Gurvits et al., 1999; Lima e Alcaniz, 2002; Daly e Guerra, 2002), entre outros. Esse surpreendente resultado se equipara em importância à descoberta da CMB.

O candidato à energia escura mais simples é a constante cosmológica $\Lambda$ cuja existência é frequentemente associada à densidade de energia do vácuo $\left(\rho_{v} \equiv \Lambda / 8 \pi G\right)$. Nesse contexto surgiu o atual modelo padrão, o chamado modelo de concordância cósmica ou modelo $\Lambda C D M$, formado por matéria bariônica, radiação, matéria escura e energia do vácuo/constante cosmológica. Apesar de seu grande poder de explicação, o modelo apre- 
senta diversas dificuldades, sendo a principal delas, a determinação da natureza da constante cosmológica e a enorme discrepância entre as estimativas teóricas e observacionais de sua densidade de energia (Weinberg, 1989; Carvalho et al., 1992; Lima e Maia, 1994).

Essas dificuldades são fontes de intensos debates na comunidade científica e abrem espaço para a discussão de propostas alternativas que sejam capazes de descrever as observações cosmológicas. Entre os modelos formulados dentro do princípio cosmológico, as principais alternativas propostas na literatura podem ser classificadas em (i) Cenários fora da TRG. como as teorias $f(R)$ (Capozziello et al.,. 2003: Carroll et al., 2004: Carvalho et al., 2008), teorias com dimensões extras acessíveis apenas pela gravidade como na teoria de Randall-Sundrum (Randall e Sundrum, 1999a.bb; Arkani-Hamed et al., 1999: Pires et al., 2006) e (ii) Modelos dentro do cenário relativístico padrão que, além do próprio modelo padrão, incluem os modelos de decaimento do vácuo, matéria-X, gás de Chaplygin, modelos de quintessência, modelos com criação de matéria escura (CCDM), entre outros.

Em linhas gerais, a estrutura e desenvolvimento desta tese são descritos a seguir.

No capítulo 2, realizamos uma breve revisão de aspectos fundamentais do modelo padrão em cosmologia. Iniciamos por discutir alguns fundamentos básicos da Teoria da Relatividade Geral, o princípio cosmológico e a métrica de Friedmann-Lemaître-RobertsonWalker. Apresentamos algumas das observações fundamentais em cosmologia e os modelos de Big-Bang e $\Lambda$ CDM.

No capítulo 3, discutimos os principais problemas associados à constante cosmológica $\Lambda$ e à energia do vácuo. Apresentamos ainda algumas das diversas alternativas para a energia escura discutidas na literatura.

No capítulo 4 descrevemos a termodinâmica e dinâmica da formulação macroscópica da criação de partículas no contexto cosmológico. Mostramos que o processo de criação de partículas é um processo irreversível que pode ser contabilizado nas equações de campo de Einstein através de um termo de pressão. Assumindo que a entropia por partícula permaneça constante durante a criação, é demonstrado que o processo de criação de partículas gera um termo de pressão negativa que, em princípio, pode acelerar o universo.

No capítulo 5, apresentamos uma proposta original de modelo com criação de matéria escura (CCDM - Creation of Cold Dark Matter). Derivamos a dinâmica básica do universo nos casos de criação de matéria escura fria e com pressão no chamado modelo de Lima, Jesus \& Oliveira (LJO). Vinculamos os parâmetros livres do modelo através de um teste 
estatístico de $\chi^{2}$ utilizando dados de Supernovas do Tipo Ia. Destacamos por fim a equivalência completa entre os modelos LJO e $\Lambda$ CDM em nível de background, ainda que cada modelo possua hipóteses básicas completamente distintas.

As perturbações lineares do modelo LJO são discutidas no capítulo 6. Iniciamos esse capítulo discutindo a formulação Neo-Newtoniana e, posteriormente, desenvolvendo o formalismo para perturbações lineares de densidade no modelo LJO. Investigamos o papel da velocidade específica do som, $c_{e f f}^{2}$, na formação de estruturas nesse contexto. Os parâmetros livres foram limitados através de um teste de $\chi^{2}$ para dados da taxa de crescimento e algumas formas funcionais da velocidade efetiva do som. Discutimos ainda diferentes metodologias na literatura para a análise das perturbações de densidade nos modelos CCDM e a chamada "degenerescência escura".

No capítulo 7, apresentamos um novo modelo CCDM não degenerado com os modelos $\Lambda$ CDM e LJO e ainda capaz de explicar a aceleração cósmica. Realizamos uma análise estatística utilizando dados do parâmetro de Hubble em diferentes redshifts e SN Ia.

No capítulo 8, discutimos a possibilidade da origem da expansão cósmica acelerada em um modelo com decaimento do vácuo e sua descrição através de um campo escalar. Nesse capítulo, ainda apresentamos resultados acerca do comportamento termodinâmico do modelo. Vinculamos os seus parâmetros livres utilizando dados de SNe Ia e da chamada razão $\mathrm{CMB} / \mathrm{BAO}$.

Finalmente, apresentamos as nossas conclusões e algumas das perspectivas para o desenvolvimento futuro da cosmologia, no capítulo 9 .

Com o intuito de facilitar a consulta de estudantes e pesquisadores, informamos que as contribuições originais desta Tese estão contidas nos capítulos 4, 6, 7 e 8 e podem ser consultadas nos trabalhos Lima et al. (2010), Lima et al. (2011), Jesus et al. (2011), Andrade-Oliveira, F. et al. (2014) e Jesus e Andrade-Oliveira, F. (2015). 
Capítulo 2

\section{Cosmologia Física}

\subsection{Introdução}

Parte importante do estudo do universo como um todo está na elaboração de modelos e na realização de testes observacionais. O cenário que melhor descreve as observações cosmológicas é o modelo de Big Bang, no qual o universo vem se expandindo e se resfriando desde um passado de condições extremas até o momento atual. Esse cenário tem em suas bases teóricas a Teoria da Relatividade Geral e o princípio cosmológico e vem produzindo previsões confirmadas por diversas observações como, por exemplo, a expansão do universo, abundância de núcleos leves sintetizados nos seus primeiros minutos de existência e a existência da radiação cósmica de fundo.

Neste capítulo, são apresentados alguns aspectos relevantes das teorias em que esta tese se fundamenta, iniciando pela Teoria da Relatividade Geral e pela cosmologia relativística. O conteúdo mostrado aqui pode ser encontrado de forma mais detalhada em livros textos de relatividade geral e cosmologia relativística, como por exemplo, em Weinberg (1972); Mukhanov (2005); Amendola e Tsujikawa (2010); Ellis et al. (2012), entre outros.

\subsection{Teoria da Relatividade Geral}

A Teoria da Relatividade Geral (TRG), proposta por Albert Einstein em 1915, reinterpretou os fenômenos gravitacionais, os conciliando com as ideias previamente apresentadas na Relatividade Restrita. O passo fundamental para o seu desenvolvimento foi dado em 1907, quando Einstein elaborou o chamado princípio da equivalência entre gravitação e matéria ou apenas princípio da equivalência (PE).

Desde o início do século XVII, com os experimentos de queda livre de Galileo Galilei, é 
conhecido o fato de que os corpos na superfície da Terra se movem sob igual aceleração no vácuo, independentemente de suas massas. Com o surgimento da mecânica Newtoniana, essa característica pôde ser interpretada como a equivalência entre a massa gravitacional $\left(m_{G}\right)$ e a massa inercial $\left(m_{i}\right)$. Entretanto, o próprio Isaac Newton já chamava a atenção de que não haveria nenhuma razão a priori para essa igualdade entre massas. Ciente disso, Newton realizou alguns experimentos com medidas de períodos em pêndulos de diferentes materiais chegando à conclusão de que, se o princípio não fosse válido, a diferença entre as massas seria inferior a $10^{-3}$. Em 1889, utilizando um experimento com pêndulos de torção, Roland Eötvös mostrou que o limite máximo para uma eventual discrepância era $10^{-9}$. Experimentos atuais mostram que o princípio permanece válido em pelo menos $1-m_{G} / m_{i} \leq 10^{-14}$ (Turyshev, 2008).

Com base nessa equivalência, Einstein partiu da premissa de que seria impossível distinguir localmente entre a aceleração de origem gravitacional e a aceleração causada por um referencial não inercial. Sob a força da gravidade, quaisquer partículas de teste sujeitas às mesmas condições iniciais sofrerão os mesmos efeitos, independentemente de suas propriedades - incluindo suas massas de repouso. Assim, a TRG estabelece um lugar especial para a gravidade entre as forças fundamentais da natureza, uma vez que o comportamento de um corpo de teste sob a ação de um campo gravitacional é interpretado como um efeito puramente geométrico.

Em 1907, Einstein utilizou o princípio da equivalência para calcular o efeito de desvio para o vermelho sofrido pela luz sujeita a um campo gravitacional, demonstrando que era possível prever a ação da gravidade sobre um sistema físico arbitrário. Entretanto, entre esse passo inicial e a formulação final da TRG era necessário entender como se formam os campos gravitacionais. Em 1913, Einstein e o matemático Marcel Grosmann associaram o campo gravitacional às 10 componentes independentes do tensor métrico de Riemann, obtendo a formulação tensorial da gravitação que conhecemos hoje.

A geometria de um sistema físico é determinada pela sua métrica. Na Relatividade Restrita, o elemento de linha é dado por $(c=1)$ :

$$
d s^{2}=d t^{2}-d x^{2}-d y^{2}-d z^{2}
$$

sendo $t$ a coordenada temporal e $x, y$ e $z$ as coordenadas espaciais. De forma sucinta, a expressão acima pode ser reescrita como: 


$$
d s^{2}=\eta_{\mu \nu} d x^{\mu} d x^{\nu}
$$

onde $\eta_{\mu \nu} \equiv$ Diagonal $\left(+--_{-}\right)$é o tensor métrico do espaço-tempo de Minkowski e $x^{\mu}$ representa as coordenadas espaço-temporais.

Na TRG, a presença de um campo de matéria induz uma curvatura no espaço-tempo. Assim, a geometria modificada do sistema físico está codificada no novo elemento de linha (Weinberg, 1972):

$$
d s^{2}=g_{\mu \nu} d x^{\mu} d x^{\nu}
$$

onde $g_{\mu \nu}$, o tensor métrico do espaço-tempo curvo, determina todas as propriedades geométricas do sistema de coordenadas curvilíneo (Landau e Lifshitz, 1971).

Definido o tensor métrico do sistema físico, o tensor de Einstein $G_{\mu \nu}$ pode ser escrito como

$$
G_{\mu \nu}=R_{\mu \nu}-\frac{1}{2} g_{\mu \nu} R
$$

onde $R_{\mu \nu} \equiv R_{\mu \alpha \nu}^{\alpha}$ é o tensor de Ricci, obtido através da contração do tensor de RiemannChristoffel (Weinberg, 1972)

$$
R_{\mu \beta \nu}^{\alpha}=\frac{\partial \Gamma_{\mu \nu}^{\alpha}}{\partial x^{\beta}}-\frac{\partial \Gamma_{\mu \beta}^{\alpha}}{\partial x^{\nu}}+\Gamma_{\lambda \beta}^{\alpha} \Gamma_{\mu \nu}^{\lambda}-\Gamma_{\lambda \nu}^{\alpha} \Gamma_{\mu \beta}^{\lambda},
$$

no qual $\Gamma_{\mu \nu}^{\alpha}$ são os símbolos de Christoffel que, em termos da métrica, são dados por:

$$
\Gamma_{\mu \nu}^{\alpha}=\frac{1}{2} g^{\alpha \lambda}\left[\frac{\partial g_{\lambda \mu}}{\partial x^{\nu}}+\frac{\partial g_{\nu \lambda}}{\partial x^{\mu}}-\frac{\partial g_{\mu \nu}}{\partial x^{\lambda}}\right]
$$

A geometria do espaço-tempo e o conteúdo massa-energia estão interligados através das equações de campo de Einstein:

$$
G_{\mu \nu}-\Lambda g_{\mu \nu}=\chi T_{\mu \nu}
$$

onde $G_{\mu \nu}$ é o tensor de Einstein e $T_{\mu \nu}$ é o tensor energia-momento. A constante $\chi \equiv$ $8 \pi G / c^{4}$ é a constante de Einstein, obtida no limite de campos fracos. Na equação acima, o lado esquerdo contém as propriedades geométricas do espaço-tempo enquanto o lado direito contém a descrição da energia e momento do conteúdo material. O termo $\Lambda g^{\mu \nu}$ foi introduzido por Einstein inspirado por um modelo cosmológico no qual $\Lambda$ é a chamada constante cosmológica. 
Quando a fonte de energia-momento em (2.7) é um fluido perfeito, o conteúdo do tensor energia-momento é completamente caracterizado por duas variáveis termodinâmicas: a densidade de massa-energia $\rho$ e a pressão isotrópica $p$. Consideremos o tensor $\tilde{T}^{\mu \nu}$ como o tensor energia-momento em um sistema de referência localmente lorentziano em um dado ponto $x$ do espaço-tempo. Nesse ponto em particular, a componente $\tilde{T}^{00}$ representa a densidade de massa energia (Weinberg, 1972):

$$
\tilde{T}^{00}=\rho,
$$

enquanto as componentes diagonais $\tilde{T}^{i i}$ são

$$
\tilde{T}^{i i}=p
$$

Além disso, como estamos descrevendo um fluido perfeito, a ausência de condução de calor ou viscosidade impõe as condições $\tilde{T}^{i 0}=\tilde{T}^{0 j}=0$, e $\tilde{T}^{i j}=0$ se $i \neq j$.

Sendo $u^{\mu}=\delta_{0}^{\mu}$ para um observador comóvel, o tensor $\tilde{T}^{\mu \nu}$ pode ser escrito de forma manifestamente covariante como:

$$
\tilde{T}^{\mu \nu}=(\rho+p) u^{\mu} u^{\nu}-p \eta^{\mu \nu}
$$

Implementando os efeitos gravitacionais através do acoplamento mínimo (Weinberg, 1972), o tensor momento-energia tem a forma

$$
T^{\mu \nu}=(\rho+p) u^{\mu} u^{\nu}-p g^{\mu \nu}
$$

e satisfaz à seguinte identidade:

$$
T^{\mu \nu}{ }_{\nu}=0
$$

A identidade (2.12) estabelece a forma local das leis de conservação de energia e de momento, de modo independente do sistema de coordenadas adotado.

Assumindo a TRG como melhor teoria de gravitação disponível atualmente, as equações de campo de Einstein são o primeiro passo na formulação de modelos cosmológicos. A seguir, discutiremos as hipóteses físicas dos modelos que servirão de base para a descrição da dinâmica do universo. 


\subsection{Métrica de Friedmann-Lemaître-Robertson-Walker}

O Princípio Cosmológico estabelece que não existam observadores privilegiados no universo. Isso quer dizer que para cada instante de tempo $t$ o universo deve parecer igual a todos os observadores. Como consequência, o universo deve ser homogêneo e isotrópico, ao menos em grandes escalas. Esse princípio, fortemente sustentado pelas observações da radiação cósmica de fundo e do universo em grandes escalas, tem suas propriedades geométricas sintetizadas na métrica de Friedmann-Lemaittre-Robertson-Walker (FLWR), cujo elemento de linha é dado por (Weinberg, 1972):

$$
d s^{2}=d t^{2}-a^{2}(t)\left(\frac{d r^{2}}{1-K r^{2}}+r^{2} d \theta^{2}+r^{2} \sin ^{2} \theta d \phi^{2}\right)
$$

onde $r, \phi$ e $\theta$ são coordenadas esféricas comóveis, t é o tempo próprio, $a(t)$ é o fator de escala cósmico, K é o parâmetro de curvatura da seção espacial do universo, podendo assumir os valores $K=+1$ (universo fechado), $K=0$ (universo plano) e $K=-1$ (universo hiperbólico).

\subsection{Dinâmica Cosmológica}

Para um tensor energia-momento da forma (2.11), as equações de campo de Einstein (2.7) na métrica de FLRW (2.13) tomam a forma (Friedmann, 1922, 1924; Weinberg, 1972):

$$
\begin{gathered}
8 \pi G \rho+\Lambda=3\left(\frac{\dot{a}}{a}\right)^{2}+3 \frac{K}{a^{2}}, \\
8 \pi G p-\Lambda=-2 \frac{\ddot{a}}{a}-\left(\frac{\dot{a}}{a}\right)^{2}-\frac{K}{a^{2}},
\end{gathered}
$$

onde cada ponto $\left({ }^{*}\right)$ representa uma derivada em relação ao tempo, $\rho=\sum_{i} \rho_{i}$ é a soma das densidades individuais dos diferentes tipos de fluidos que compõe o modelo e $p=\sum_{i} p_{i}$ é a pressão total do sistema.

O sistema de equações (2.14) e (2.15) é conhecido como equações de Friedmann e é ele que rege a dinâmica do fator de escala do universo. A constante cosmológica $\Lambda$ foi originalmente introduzida por Einstein com o intuito de produzir um modelo de universo estático $(\ddot{a}=\dot{a}=0)$ e com curvatura positiva $(K>0)$. 
Além disso, pode-se demonstrar que as equações de Friedmann (2.14|2.15) contêm a conservação local de energia:

$$
\dot{\rho}+3 \frac{\dot{a}}{a}(\rho+p)=0
$$

Boa parte dos fluidos de interesse cosmológico possui uma equação de estado barotrópica do tipo $p_{i}=\omega_{i} \rho_{i}$, onde $\omega_{i}$ é chamado de parâmetro da equação de estado. Sempre que não ocorra conversão entre diferentes tipos de fluidos, a equação (2.16) pode ser facilmente integrada, descrevendo a evolução da densidade de energia do fluido como

$$
\rho_{i}=\rho_{i 0}\left(\frac{a}{a_{0}}\right)^{-3\left(1+\omega_{i}\right)},
$$

onde $a_{0}$ é o fator de escala hoje e $\rho_{i 0}$ é o valor da densidade de energia hoje.

Podemos agora analisar a evolução da densidade para alguns fluidos de interesse. Para a matéria não-relativística, caso da matéria escura fria e dos bárions, a pressão do fluido é considerada desprezível. Logo, para $\omega=0$,

$$
\rho_{m}=\rho_{m 0}\left(\frac{a}{a_{0}}\right)^{-3},
$$

onde $\rho_{m 0}$ é densidade da matéria medida hoje. A matéria relativística, como no caso da radiação, tem equação de estado $p=1 / 3 \rho$, nos levando à solução

$$
\rho_{r}=\rho_{r 0}\left(\frac{a}{a_{0}}\right)^{-4},
$$

onde $\rho_{r 0}$ é o valor atual da densidade de radiação.

A constante cosmológica pode ser caracterizada por um fluido com equação de estado $p=-\rho$. Logo, a sua densidade de energia é simplesmente

$$
\rho_{\Lambda}=\frac{\Lambda}{8 \pi G}=\text { constante }
$$

\subsection{Parâmetros Cosmológicos}

Em geral, modelos cosmológicos são caracterizados por alguns parâmetros cosmológicos básicos que caracterizam e distinguem diferentes modelos. Entre os mais úteis temos o 
parâmetro de Hubble $H(z)$, os parâmetros de densidade e o parâmetro de desaceleração, conforme discutiremos a seguir.

A taxa de variação do volume comóvel $V$ é determinada pelo parâmetro de Hubble:

$$
H(t) \equiv \frac{1}{a} \frac{d a}{d t}=\frac{1}{V} \frac{d V}{d t}
$$

cujo valor medido localmente $\left(t=t_{0}\right)$ é a constante de Hubble, $H_{0}$.

A densidade crítica do universo, $\rho_{c}$, é definida como o valor de densidade total para um universo plano. Da equação (2.14), com $K=0$, temos:

$$
\rho_{c} \equiv \frac{3 H^{2}}{8 \pi G},
$$

cujo valor atual $\rho_{c 0}$ é dado por:

$$
\rho_{c 0} \equiv \frac{3 H_{0}^{2}}{8 \pi G}=1.8788 \cdot 10^{-29} h^{2} \frac{g}{\mathrm{~cm}^{3}} .
$$

Considerando-se as definições acima, o parâmetro de densidade atual de uma dada componente do universo, é dado pela razão entre sua densidade hoje $\rho_{i 0}$ e $\rho_{c 0}$, isto é :

$$
\Omega_{i}=\frac{\rho_{i 0}}{\rho_{c 0}}
$$

De forma análoga, o valor atual do parâmetro de densidade de curvatura é definido como:

$$
\Omega_{k}=-\frac{K}{a_{0}^{2} H_{0}^{2}} .
$$

Utilizando a equação (2.14), podemos mostrar que os parâmetros de densidade satisfazem à relação:

$$
1=\Omega_{k}+\sum_{i} \Omega_{i}
$$

onde a somatória é realizada sobre todas as componentes de densidade incorporadas no modelo cosmológico. Dessa forma, dentro das equações de Friedmann, é estabelecida a relação entre a curvatura e o conteúdo energético-material total do universo:

$$
\begin{array}{rlll}
\sum_{i} \Omega_{i}>1 & \Longleftrightarrow K>0 & \text { Universo Fechado; } \\
\sum_{i} \Omega_{i}=1 & \Longleftrightarrow K=0 & \text { Universo Plano; } \\
\sum_{i} \Omega_{i}<1 & \Longleftrightarrow K<0 & \text { Universo Hiperbólico. }
\end{array}
$$


Em diversos casos, o parâmetro de Hubble pode ser então expresso em termos da constante de Hubble, $H_{0}$, e dos parâmetros de densidades definidos acima. Por exemplo, no modelo composto de matéria relativística, matéria não-relativística, constante cosmológica e com curvatura arbitrária, temos (Weinberg, 2008):

$$
H^{2}=H_{0}^{2}\left[\Omega_{m}\left(\frac{a_{0}}{a}\right)^{3}+\Omega_{r}\left(\frac{a_{0}}{a}\right)^{4}+\Omega_{\Lambda}+\Omega_{k}\left(\frac{a_{0}}{a}\right)^{2}\right]
$$

onde $\Omega_{m}, \Omega_{r}$ e $\Omega_{\Lambda}$ são os parâmetros de densidade da matéria não-relativística, relativística e constante cosmológica, respectivamente.

Outro importante indicador dinâmico do universo é o parâmetro de desaceleração, responsável por determinar a taxa de aceleração ou de desaceleração do universo, definido por:

$$
q(t) \equiv-\frac{\ddot{a} a}{\dot{a}^{2}}
$$

O parâmetro de desaceleração hoje, $q_{0}$, é definido como o valor atual do parâmetro acima. Valores $q_{0}<0$ indicam que o universo está passando por uma fase de expansão acelerada, enquanto $q_{0}>0$ indica que o universo passa por uma expansão desacelerada.

\subsection{Redshift}

O desvio para o vermelho z, conhecido como redshift, é uma das medidas mais importantes no estudo do universo, pois é um observável diretamente associado ao fator de escala cósmico. Adotando a métrica (2.13), na qual a Terra se situa na origem do sistema de coordenadas, vamos considerar um pico de onda eletromagnética emitido em um dado instante $t_{e}$ por uma fonte comóvel com coordenadas $\left(r_{1}, \phi, \theta\right)$. A onda eletromagnética viaja ao longo de uma geodésica nula por coordenadas $\phi$ e $\theta$ constantes de modo que, para uma onda se movendo em direção à Terra:

$$
\int_{t_{e}}^{t_{0}} d t=\int_{0}^{r_{1}} \frac{d r}{\sqrt{1-K r^{2}}}=f\left(r_{1}\right)
$$

sendo $t_{0}$ o instante em que a onda é observada. Se o próximo pico de onda for emitido pela fonte em $t_{e}+\delta t_{e}$, então:

$$
\int_{t_{e}+\delta t_{e}}^{t_{0}+\delta t_{0}} \frac{d t}{a(t)}=f\left(r_{1}\right)
$$

A coordenada comóvel da fonte $r_{1}$ permanece constante no tempo e, portanto, o mesmo ocorre a $f\left(r_{1}\right)$. Em uma escala de tempo típica para a emissão de dois picos consecutivos 
de uma onda eletromagnética ( $10^{-14} \mathrm{~s}$ ), a variação do fator de escala é desprezível. Dessa forma, comparando-se as equações (2.30) e (2.31), temos que (Weinberg, 1972, 2008):

$$
\frac{\delta t_{0}}{a\left(t_{0}\right)}=\frac{\delta t_{e}}{a\left(t_{e}\right)}
$$

logo, a relação entre a frequência emitida pela fonte e a observada é:

$$
\frac{\nu_{e}}{\nu_{0}}=\frac{a\left(t_{0}\right)}{a\left(t_{e}\right)} \equiv \frac{a_{0}}{a_{e}}
$$

onde $\nu_{e}$ e $\nu_{0}$ são as frequências emitida e observada, respectivamente.

O redshift $z$ é definido como:

$$
z:=\frac{\lambda_{0}-\lambda_{e}}{\lambda_{e}}=\frac{a_{0}}{a_{e}}-1
$$

onde $\lambda_{0}$ é a frequência observada, $\lambda_{e}$ é a frequência emitida e $\lambda_{0} / \lambda_{e}=\nu_{e} / \nu_{0}$.

\subsection{Modelos de Big Bang}

O modelo de Big Bang descreve um universo cuja expansão se iniciou há aproximadamente 14 bilhões de anos, a partir de um estado extremamente quente e denso até o universo atual. Esse cenário é apoiado por quatro pilares principais.

O formalismo teórico do modelo é baseado na Teoria da Relatividade Geral e no princípio cosmológico, tendo sua dinâmica descrita pelas equações de Friedmann. Observacionalmente, o modelo é sustentado pela expansão do universo, originalmente observada por (Lemaître, 1927; Hubble, 1929), pelas previsões relacionadas à síntese primordial dos elementos leves, durante os primeiros minutos após o Big Bang (Gamow, 1946) e pela existência de um fundo de radiação em micro-ondas com espectro de corpo negro permeando o universo, a chama radiacão cósmica de fundo. observada originalmente por Penzias e Wilson (1965).

\subsubsection{A Expansão do Universo}

As primeiras soluções cosmológicas expansionistas foram obtidas pelo matemático e meteorologista Alexander Friedmann (1922, 1924). Na mesma década, foram discutidas as observações do afastamento de objetos distantes por Georges Lemaître (1927) e, independentemente, por Edwin Hubble (1929). 
Em particular, Hubble estudou o espectro de emissão de dezenas de galáxias chegando à conclusão de que havia um desvio sistemático para o vermelho de linhas espectrais desses objetos. Além disso, a lei de afastamento observada era aproximadamente linear, isto é,

$$
v=H_{0} d,
$$

onde $v$ é a velocidade de recessão do objeto, $d$ é sua distância e $H_{0}$ é a constante de Hubble. A lei (2.35) recebe o nome de lei de Hubble. Estimativas baseadas em observações locais, tem como valor para a constante de Hubble $H_{0}=72.0 \pm 3.0 \mathrm{~km} \mathrm{~s}^{-1} \mathrm{Mpc}^{-1}$ Humphreys et al. (2013).

Dentro da TRG, o universo em expansão é descrito através do aumento do fator de escala $a(t)$, enquanto sua dinâmica é obtida através das equações de Friedmann (2.14)-(2.15). A variação do fator de escala $a(t)$ é diretamente determinada pelo conteúdo energético do universo. A expansão do universo foi umas das descobertas mais importantes do século XX, rompendo com o pensamento corrente até então de que o universo seria estático.

Tão importante quanto a descoberta de que o universo não é estático é a consequente conclusão de que este teve uma origem. O afastamento das galáxias e a expansão do universo são indicações claras de que no passado o fator de escala foi muito menor. Como extrapolação, podemos retroceder nos modelos de Friedmann até o momento em que a distância entre quaisquer dois pontos tende a zero. Esse limite, quando a densidade do universo tende a infinito, é conhecido como singularidade inicial e define um instante inicial $t \rightarrow 0$. Acreditamos hoje que essa situação extrema extrapole os limites de validade da TRG e que no futuro esse problema possa ser contornado por uma teoria quântica da gravitação.

\subsubsection{Nucleossíntese Primordial}

Nos primeiros minutos após o Big Bang, o universo era uma sopa cósmica quente e densa, cuja componente dominante era a radiação. As partículas nucleares no plasma primordial, portanto, deveriam estar muito mais sujeitas a interações do que os constituintes do universo estão hoje. A expansão do universo resfriou esse plasma, dando origem a processos fora de equilíbrio. O estudo da formação de núcleos leves no universo ainda jovem, a nucleossíntese primordial, tem como principais ingredientes as razões entre número de fótons e de bárions e entre prótons e nêutrons. 
Os cálculos da síntese dos elementos mais leves no universo primordial dependem ainda da temperatura, da taxa de expansão e das taxas de reações fracas e fortes. A interação fraca é determinante nas reações de interconversão entre nêutrons e prótons que, por sua vez, influenciam a síntese do ${ }^{4} \mathrm{He}$. Já as reações nucleares determinam a razão fóton-bárion $\eta$, o número de espécies de neutrino $N_{\nu}$ assim como a produção e destruição de elementos leves.

Nessa teoria, a expansão do universo teve papel fundamental na geração de elementos em condições de não-equilíbrio. A produção dos elementos leves se deu entre os instantes $t \approx 10^{-2} s$ e $t \approx 10^{2} s$ após a singularidade inicial, quando a temperatura do universo variou de $T \approx 10 \mathrm{MeV}$ para $T \approx 10^{-1} \mathrm{MeV}$. Nesse poucos segundos, houve a formação abundante de deutério $\left({ }^{2} H\right)$, hélio-3 $\left({ }^{3} \mathrm{He}\right)$, hélio $\left({ }^{4} \mathrm{He}\right)$ e lítio $\left({ }^{7} \mathrm{Li}\right)$. Com a queda temperatura, a pressão no plasma primordial deixou de ser suficiente para a manutenção das reações nucleares, cessando a síntese dos elementos leves. Assim, espera-se que as abundâncias relativas dos elementos leves observadas estejam essencialmente fixas desde então.

A nucleossíntese primordial prevê que a parte bariônica do universo seja formada por aproximadamente $75 \%$ de hidrogênio, $24 \%$ de hélio e menos de $1 \%$ de outros elementos (Aver et al., 2013). Por outro lado, a abundância observada dos núcleos mais pesados não pode ser explicada em função dessa teoria, uma vez que a ausência de núcleos estáveis com massa atômica entre 5 e 8 não permite uma taxa significativa de fusões no universo primordial, necessária a esses elementos. Esse problema foi posteriormente resolvido por Burbidge et al. (1957) através da teoria da nucleossíntese estelar, onde se mostrou que as condições interiores das estrelas seriam ideais para a formação dos elementos mais pesados. Entretanto, a abundância de lítio prevista pela nucleossíntese primordial excede em aproximadamente 3 vezes o valor inferido observacionalmente, permanecendo uma questão em aberto à teoria (Steigman, 2012).

A teoria da nucleossíntese primordial é fundamentada no modelo padrão de física de partículas, gerando previsões muito confiáveis sobre o universo primordial. As estimativas de abundâncias relativas dos elementos leves estão entre os mais importantes testes cosmológicos. 


\subsubsection{Radiação Cósmica de Fundo}

As observações da Radiação Cósmica de Fundo em Micro-ondas (Cosmic Microwave Background - CMB) formam um retrato do universo extremamente jovem, com aproximadamente 380.000 anos de idade, sendo um dos principais "registros fósseis" do universo. A história de sua previsão se confunde com a história da formulação da teoria da nucleossíntese primordial.

Quando Gamow e colaboradores propuseram os primeiros cálculos da nucleossíntese primordial, seu aluno R. Alpher encontrou uma inconsistência relacionada à taxa de criação dos elementos mais pesados. De modo simplificado, a condição para a síntese apreciável mas não excessiva de elementos mais pesados, durante o tempo de expansão $t$ e para a densidade de bárions $n$ é (Peebles, 2012),

$$
\text { ovnt } \sim 1
$$

onde $\sigma$ é a seção de choque para a captura de nêutrons e $v$ a velocidade relativa. A escala de tempo $t$, associada à expansão gravitacional, pode ser estimada como sendo da ordem de $t \sim(G \rho)^{-1 / 2}$ na qual, em um universo dominado por matéria, $\rho=m n$. Sendo a grandeza $\sigma v$ medida independentemente, a escala de tempo $t$ obtida nessas condições tem valor muito maior que a meia vida dos nêutrons. Uma solução direta a esse problema seria supor uma criação ad hoc de nêutrons, compensando o tempo de meia vida dessas partículas.

Porém, esse problema foi contornado de forma engenhosa. Gamow supôs que o universo primordial pudesse ser tão quente a ponto de ser completamente dominado pela radiação e que, como consequência, a expansão cosmológica seria muito mais rápida do que o esperado em um universo dominado por matéria. Como subproduto desse período de condições extremas, o universo teria uma radiação de fundo remanescente, isotrópica e com temperatura atual originalmente estimada em $T_{\gamma 0} \sim 10 K$, por Alpher e Herman (1948). Entretanto, Alpher e Herman tiveram dúvidas sobre a capacidade de sobrevivência dessa radiação até os tempos atuais (Weinberg, 1972).

Já nos anos 1960, Dicke, Roll, Wilkinson e Peebles consideraram seriamente medir a temperatura dessa radiação de corpo negro através de um experimento planejado por Roll e Wilkinson. Porém, antes que o experimento se completasse, Roll e Wilkinson tomaram 
contato com as medições obtidas por dois engenheiros dos laboratórios Bell. Penzias e Wilson acidentalmente detectaram um "um excesso de temperatura de antena": um fraco sinal de fundo no comprimento de onde de $7.35 \mathrm{~cm}$, equivalente à temperatura de antena de $T_{\gamma 0}=3.5 \pm 1.0 K$ (Penzias e Wilson, 1965) .

Nas cuidadosas medidas de Penzias e WIlson, a distribuição angular da radiação detectada tornava impossível associá-la a uma fonte local de rádio ou a ruídos de origem atmosférica. A intensidade do ruído era também muitas vezes superior a qualquer erro sistemático possível, levando à conclusão de que este se tratava da radiação proveniente do Big Bang. a chamada Radiacão Cósmica de Fundo em Micro-ondas. Os artigos de Penzias e Wilson (1965) e Dicke et al. (1965) foram publicados em sequência, onde o primeiro comunicou a detecção e o segundo explicou a importância da medida realizada (Weinberg, 1972).

As principais características da CMB são seu espectro incrivelmente bem descrito por uma curva de corpo negro à temperatura de $T_{\gamma 0}=2.72548 \pm 0.00057 K$ (Fixsen, 2009), sua alta isotropia e quase homogeneidade. A isotropia sugere que a CMB seja um mar de radiação permeando o espaço de uniformemente e portanto, um observador comóvel em outra galáxia observaria essa radiação isotrópica com a mesma intensidade e temperatura, em excelente acordo com o princípio cosmológico.

A observação da CMB está associada ao limite de quão longe no passado podemos observar diretamente o universo, ao menos em ondas eletromagnéticas (Mukhanov, 2005). Para entendermos esse limite devemos retroceder nas equações de evolução de densidade. Enquanto a densidade de matéria varia como $\rho_{m} \propto(1+z)^{3}$, a densidade de radiação varia como $\rho_{r} \propto(1+z)^{4}$. Logo, pode-se inferir que houve uma época no passado em que a densidade de radiação dominou a densidade de matéria.

Nesse ambiente, quente e denso, prótons e elétrons interagiam fortemente com os fótons através do espalhamento Compton, formando o fluido fóton-bárion. As constantes interações nesse fluido não permitiam que se formassem estruturas bariônicas ou mesmos os átomos neutros. Durante a expansão cósmica e o decorrente resfriamento do universo, a interação entre radiação e matéria bariônica foi gradualmente diminuindo.

Quando o universo atingiu a idade de aproximadamente 380.000 anos, sua temperatura chegou a $T \sim 3000 K$ e os fótons abruptamente deixaram de ter energia suficiente para sustentar o processo de ionização. Com a decorrente neutralização dos átomos, os fótons 
passaram a viajar livremente, praticamente sem interação com a matéria bariônica. Esse momento, em $z \sim 1100$, é denominado de recombinação e a região a partir da qual os fótons passaram a se propagar é denominada de última superfície de espalhamento.

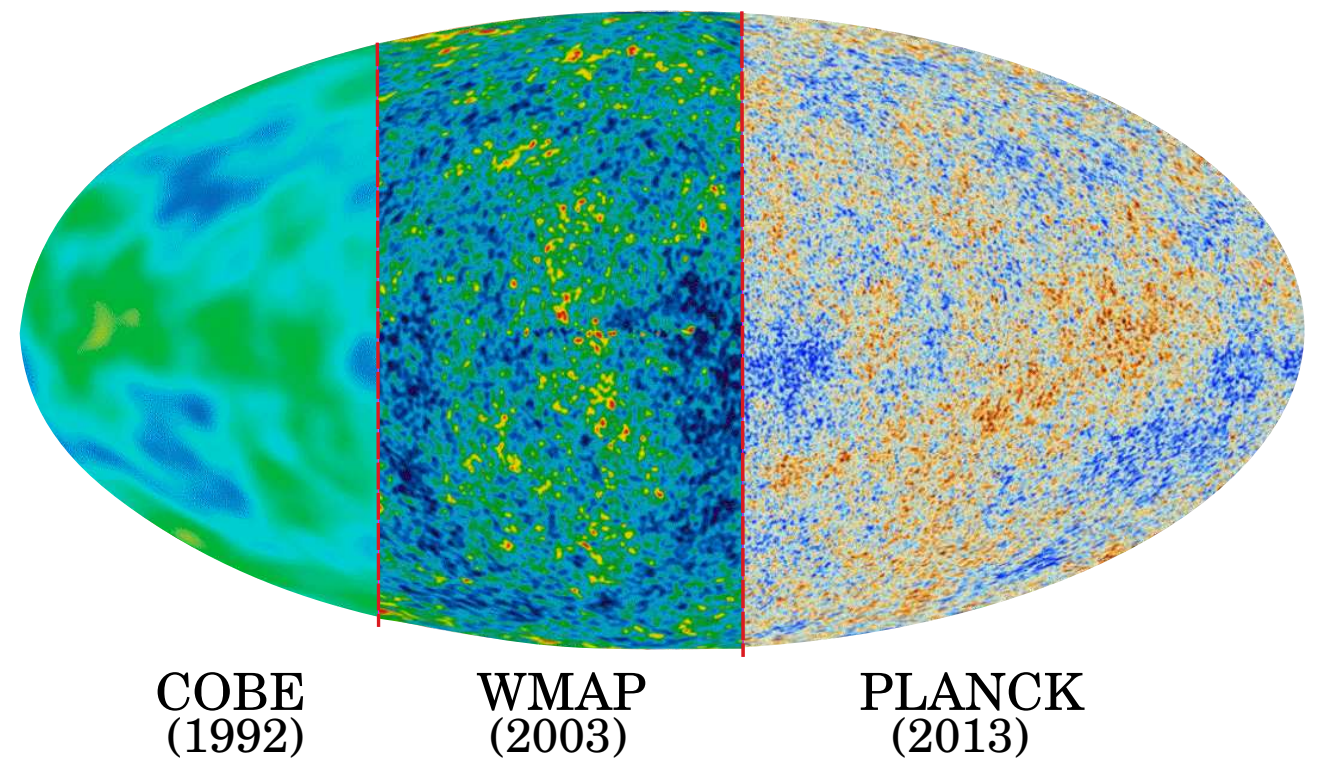

Figura 2.1: Comparacão entre os mapas do céu em micro-ondas medidos pelos proietos COBE (Smoot et al., 1992), WMAP (Hinshaw et al., 2003) e Planck (Planck Collaboration XVI: Ade. P. A. R. et al., 2013).

Os principais mapas do céu em micro-ondas foram obtidos pelo satélite COBE - Cosmic Background Explorer (Smoot et al., 1992), sendo sucedido pelo satélite WMAP - Wilkinson Microwave Anisotropy Probe (Hinshaw et al.|, 2003). Atualmente, o satélite Planck (Planck Collaboration XVI: Ade, P. A. R. et al., 2013) vêm mapeando as observações de anisotropias da CMB de todo o céu com precisão inédita (figura 2.1). Estas pequenas anisotropia (da ordem de $\delta T / T \sim 10^{-5}$ ) são geradas por perturbações do potencial gravitacional na última superfície de espalhamento e são extremamente importantes na determinação de limites sobre alguns parâmetros cosmológicos e astrofísicos (figura 2.2).

A posição do primeiro pico acústico do espectro, por exemplo, está relacionada com o tamanho do horizonte na época da recombinação, sendo bastante sensível à geometria do universo. Já o segundo pico tem sua amplitude relacionada à densidade bariônica total (inclusive os "bárions escuros"), fornecendo limites sobre o valor do parâmetro $\Omega_{b}$, enquanto o terceiro pico é mais sensível à densidade física de matéria escura $\Omega_{m} h^{2}$ (Hu e Dodelson, 2002). 


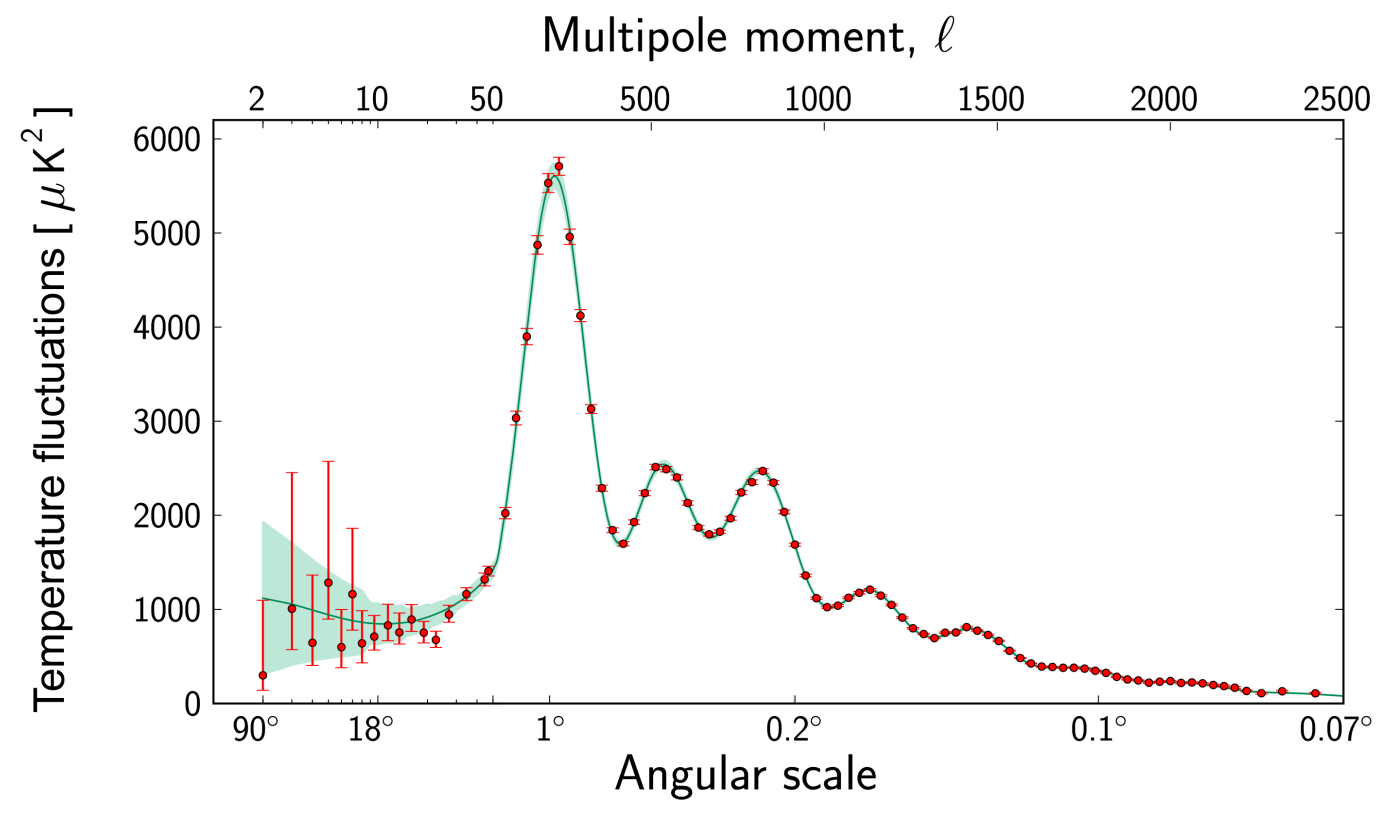

Figura 2.2: Medidas das anisotropias da CMB, realizadas por Planck Collaboration XVI: Ade, P. A. R. et al. (2013).

A radiação cósmica de fundo é uma das relíquias mais antigas do universo e que se manteve praticamente intacta por quase todo o tempo de existência do universo. A análise da informação contida nessas observações é certamente uma das mais importantes e precisas fontes de informação sobre o universo hoje e sobre suas condições no passado.

\subsubsection{Distâncias Cosmológicas}

Em um universo com curvatura e expansão, a noção euclidiana de distância deve ser repensada. Em cosmologia, trabalhamos com mais de um tipo de distância de acordo com a natureza da observação. Entre as medidas mais utilizadas estão a distância de luminosidade e a distância de diâmetro angular, discutidas a seguir.

Primeiramente, através da métrica (2.13) em um dado tempo $t$ fixo, definiremos a distância própria entre o observador e um objeto situado em $r$ como sendo (Weinberg, 1972):

$$
D_{p}=\int_{0}^{t} \frac{d t^{\prime}}{a\left(t^{\prime}\right)}
$$

Infelizmente, em escalas cosmológicas a expansão do universo não permite a obtenção 
direta dessa medida 1 sendo, portanto, necessário definirmos medidas de distância mais relevantes à cosmologia observacional.

A distância de luminosidade se baseia na relação entre a luminosidade aparente $l$ e a luminosidade absoluta $L$ de uma fonte. Consideremos um telescópio na Terra com abertura $A$ recebendo a luz de uma fonte situada no redshift $\mathrm{z}$ com luminosidade absoluta conhecida L. Em um dado instante t, a área compreendida pela frente de onda esférica se movendo em espaço-tempo com métrica diagonal dada em (2.13), é dada por (Weinberg, 1972):

$$
S=\int_{0}^{2 \pi} \int_{0}^{\pi} \sqrt{g_{\theta \theta} g_{\phi \phi}} d \theta d \phi=4 \pi a^{2}(t) r^{2}
$$

sendo $r$ a coordenada comóvel da fonte. Assim, a fração da luminosidade obtida pelo telescópio de abertura $A$ é $A /\left(4 \pi a(t) r^{2}\right)$.

Além disso, devemos considerar mais dois efeitos ligados à expansão cosmológica. Seja $t_{e}$ o momento em que a fonte emite um fóton e $t_{0}$ o momento em que este é detectado. Primeiramente, a expansão cosmológica modifica a taxa com que os fótons atingem o observador pela razão $\delta t_{0}=\delta t_{e} a\left(t_{e}\right)=\delta t_{e} /(1+z)$. Adicionalmente, cada fóton sofre o redshift cosmológico, tendo sua energia alterada por $h \nu_{0}=h \nu_{e} / a\left(t_{e}\right)=h \nu_{e}(1+z)$. Portanto, a potência P captada pelo telescópio é:

$$
P=L\left(\frac{1}{(1+z)^{2}}\right)\left(\frac{A}{4 \pi a^{2}\left(t_{0}\right) r^{2}}\right),
$$

e, consequentemente, a luminosidade aparente l é (Weinberg, 1972):

$$
l \equiv \frac{P}{A}=\frac{1}{4 \pi a^{2}\left(t_{0}\right)(1+z)^{2} r^{2} L}
$$

Em analogia com a expressão em espaço euclideano $l=L / 4 \pi d^{2}$, a distância de luminosidade $D_{L}$ em cosmologias FLRW é dada por:

$$
D_{L}=a\left(t_{0}\right)(1+z) r
$$

\footnotetext{
${ }^{1}$ Em Weinberg (1972) é descrito um experimento hipotético com o intuito de medir essa distância diretamente: Imaginemos que entre nós e um objeto de coordenada em $r_{0}$ estejam um grande número de galáxias alinhadas na nossa linha de visada, cada uma com o seu próprio observador. Em um dado tempo cósmico $t_{1}$, observadores em cada uma das galáxia medem ao mesmo tempo a distância até a próxima, medindo o tempo de viagem de um raio de luz, por exemplo. A soma de cada uma das distâncias obtidas por cada observador teria como limite o valor numérico da equação (2.37). Esse esquema seria equivalente a "congelar" o fator de escala do universo durante a realização dessa medida.
} 
Para uma fonte observada com redshift $\mathrm{z}$, a sua coordenada radial $r(z)$ pode ser obtida através da expressão:

$$
r(z)=S_{K}\left[\int_{t(z)}^{t_{0}} \frac{d t^{\prime}}{a\left(t^{\prime}\right)}\right]
$$

onde

$$
S_{K}[x] \equiv \begin{cases}\sin [x] & K=+1 \\ x & K=0 \\ \sinh [x] & K=-1\end{cases}
$$

Reescrevendo a expressão (2.42) como uma integral em z, obtemos:

$$
r(z)=\frac{1}{a_{0} H_{0} \sqrt{\left|\Omega_{k}\right|}} S_{K}\left[\sqrt{\left|\Omega_{k}\right|} \int_{0}^{z} \frac{d z^{\prime}}{E\left(z^{\prime}\right)}\right]
$$

onde $E(z) \equiv H(z) / H_{0}$. Comparando-se as expressões (2.41) e (2.44), podemos expressar a distância de luminosidade em função do redshift como:

$$
D_{L}(z)=\frac{1+z}{H_{0} \sqrt{\left|\Omega_{k}\right|}} S_{K}\left[\sqrt{\left|\Omega_{k}\right|} \int_{0}^{z} \frac{d z^{\prime}}{E\left(z^{\prime}\right)}\right]
$$

A distância de diâmetro angular estabelece a relação entre o tamanho intrínseco de um objeto e seu tamanho angular observado. A existência de barras padrão, objetos astrofísicos de dimensões conhecidas ou calculáveis, torna esta medida extremamente importante na cosmologia contemporânea.

Vamos considerar uma fonte de radiação com diâmetro próprio conhecido $s$ e com tamanha angular observado $\theta<<1$. Em analogia com a geometria euclidiana, definimos a distância de diâmetro angular $D_{A}$ como:

$$
D_{A}=\frac{s}{\theta}
$$

Assumindo que o objeto emissor esteja situado na coordenada $r$ e que a radiação observada no instante em $t_{0}$ tenha sido emitida no instante $t_{1}$, temos que, através da integração da expressão (2.13):

$$
s=a\left(t_{1}\right) \theta r .
$$

Portanto, a distância de diâmetro angular pode ser expressa como:

$$
D_{A}=a\left(t_{1}\right) r .
$$

Dada a expressão (2.48), podemos escrever a distância de diâmetro angular como: 


$$
D_{A}(z)=\frac{1}{(1+z) H_{0} \sqrt{\left|\Omega_{k}\right|}} S_{K}\left[\sqrt{\left|\Omega_{k}\right|} \int_{0}^{z} \frac{d z^{\prime}}{E\left(z^{\prime}\right)}\right] .
$$

Logo, existe uma relação simples entre as distâncias de luminosidade e de diâmetro angular, expressa por:

$$
\frac{D_{L}}{D_{A}}=(1+z)^{2}
$$

onde vemos que, para $z<<1, D_{L} \simeq D_{A} \simeq D_{p}$, conforme o esperado.

\subsubsection{Idade do Universo}

Medidas teóricas de tempo em modelos de universo são fundamentais para os estudos em cosmologia. Entende-se como a idade teórica do universo o tempo decorrido desde a singularidade inicial $(a=0)$ nas equações de Friedmann até hoje. Observacionalmente, limites inferiores para a idade do universo em um dado redshift podem ser estimados através da medição da idade de objetos antigos como aglomerados globulares, por exemplo. A comparação entre o valor teórico da idade do universo e essas medidas de idade é um teste crucial para a viabilidade de modelos cosmológicos, haja vista que a idade do universo em um dado redshift deve ser maior do que a idade de seus constituintes.

Da definição do parâmetro de Hubble (2.21) temos que:

$$
d t=\frac{d a}{\dot{a}}=\frac{d a}{a H(a)}=-\frac{d z}{(1+z) H(z)} .
$$

Definindo como tempo $t=0$ o instante em que o fator de escala tende a zero, a idade do universo em um redshift z previsto por um dado modelo pode ser calculada através da relação $(c=1)$ :

$$
t(z)=\frac{1}{H_{0}} \int_{z}^{\infty} \frac{d z^{\prime}}{\left(1+z^{\prime}\right) E\left(z^{\prime}\right)}
$$

A idade total do universo, $t_{0}$, é obtida ajustando o limite de integração para $z=0 \mathrm{em}$ (2.52), ou seja:

$$
t_{0}=\frac{1}{H_{0}} \int_{0}^{\infty} \frac{d z^{\prime}}{(1+z) E\left(z^{\prime}\right)}
$$

ou seja, estimativas teóricas da idade do universo dependem basicamente da taxa de expansão local $H_{0}$ e dos parâmetros de densidade contidos na função $E(z)$. O valor de idade baseado nas medidas de CMB dentro do modelo $\Lambda$ CDM é $t_{0}=13.798 \pm 0.037 \times 10^{9}$ anos (Planck Collaboration XVI: Ade, P. A. R. et al., 2013). 


\subsubsection{Matéria Escura}

A maior parte da informação extragaláctica a que temos acesso está na forma de radiação eletromagnética. Ainda assim, o modelo padrão em cosmologia prevê que a mais de 95\% do conteúdo energético do universo não interage por esse meio, formando o chamado setor escuro do universo. Uma das componentes desse setor é a matéria escura, cujos efeitos são indiretamente observados nas mais distintas escalas: das escalas galácticas, por meio das curvas de rotação, às escalas além do horizonte, através das anisotropias da radiação cósmica de fundo (Roos, 2010).

Conforme vem sendo discutido desde os anos 1930, modelos físicos baseados em potenciais newtonianos revelam que a massa esperada para galáxias e aglomerados de galáxias é muito superior ao valor estimado através da relação Massa-Luminosidade nestes objetos. As primeiras propostas de um termo de massa não visível são atribuídas a Zwicky (1933) e Smith (1936) quando estes tentaram estimar as massas dos aglomerados de Coma e Virgo através do teorema do virial.

Em escalas galácticas, os estudos do comportamento das curvas de rotação de galáxias espirais fornecem evidências independentes da existência do termo de matéria não-visível. Curvas de rotação relacionam a velocidade circular orbital em função da distância radial ao centro galáctico, podendo ser obtidas através de traçadores, como estrelas e nuvens de hidrogênio neutro (HI). Baseados na física newtoniana e na observação de que a maior parte da massa luminosa das galáxias se acumula em seu centro, esperava-se que a curva de rotação tivesse um comportamento como o da curva de um corpo rígido em pequenos raios e, para médios e grandes raios, o seu comportamento fosse kepleriano.

Quando Rubin e Ford (1970) mediram curvas de rotação para a galáxia M31, observaram que o comportamento esperado em pequenos raios era confirmado. Entretanto, surpreendentemente, as partes mais externas das curvas de rotação apresentaram um grande desvio da previsão kepleriana. Em médias e grandes distâncias do centro galáctico, as curvas de rotação permaneciam aproximadamente constantes. Essa característica vem sendo observada desde então em diversas outras galáxias, indicando a existência de uma componente de massa adicional à matéria luminosa e dominante em suas regiões mais externas, formando um halo escuro (figura 2.3).

Em aglomerados de galáxias, estima-se que a matéria na forma de gás excede em pelo 


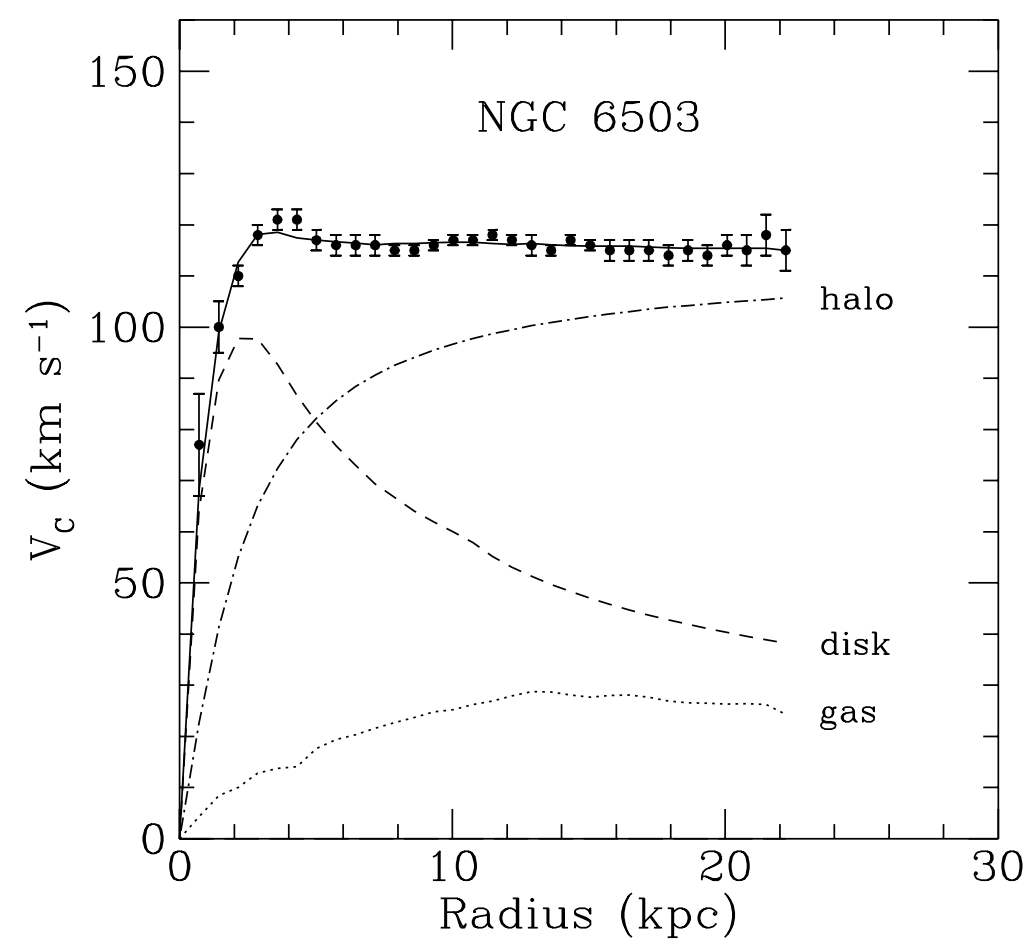

Figura 2.3: Curva de rotação da galáxia espiral NGC6503. Os pontos representam medidas da velocidade de rotação em função da distância ao centro galáctico. As linhas representam as contribuições para a velocidade rotacional de cada uma das componentes visíveis (disco e gás) e da componente escura (halo) da galáxia. A adição de uma componente de halo permite o ajuste das observações de velocidade, sendo mais importante para grandes distâncias do centro galáctico. Figura retirada de Begeman et al. (1991).

menos uma ordem de magnitude a contribuição na forma de estrelas (Forman e Jones, 1982; White et al., 1993; Evrard, 1997; Lin et al., 2003; Gonzalez et al., 2007; Sun et al., 2009; Giodini et al., 2009; Laganá et al., 2013). Desse modo, a massa em forma de gás é uma estimativa razoável da massa bariônica total de um aglomerado. White et al. (1993), através da medida de 19 aglomerados, estimaram que a razão entre massa do gás e massa dinâmica nos aglomerados está contida no limite [0.15, 0.41], apontando para um problema de massa faltante também nesses objetos.

Uma evidência particularmente interessante vem das observações do aglomerado 1E0657558, o chamado Aglomerado da Bala (Bullet Cluster). Esse objeto é resultado do processo de fusão em andamento de dois aglomerados, formando um sistema com quatro componentes observacionalmente distintas: uma componente de gás emitindo em raios-X e uma componente estelar para cada um dos objetos na fusão (Tucker et al., 1998). Além disso, estas componentes se encontram espacialmente segregadas. Durante o processo de colisão 

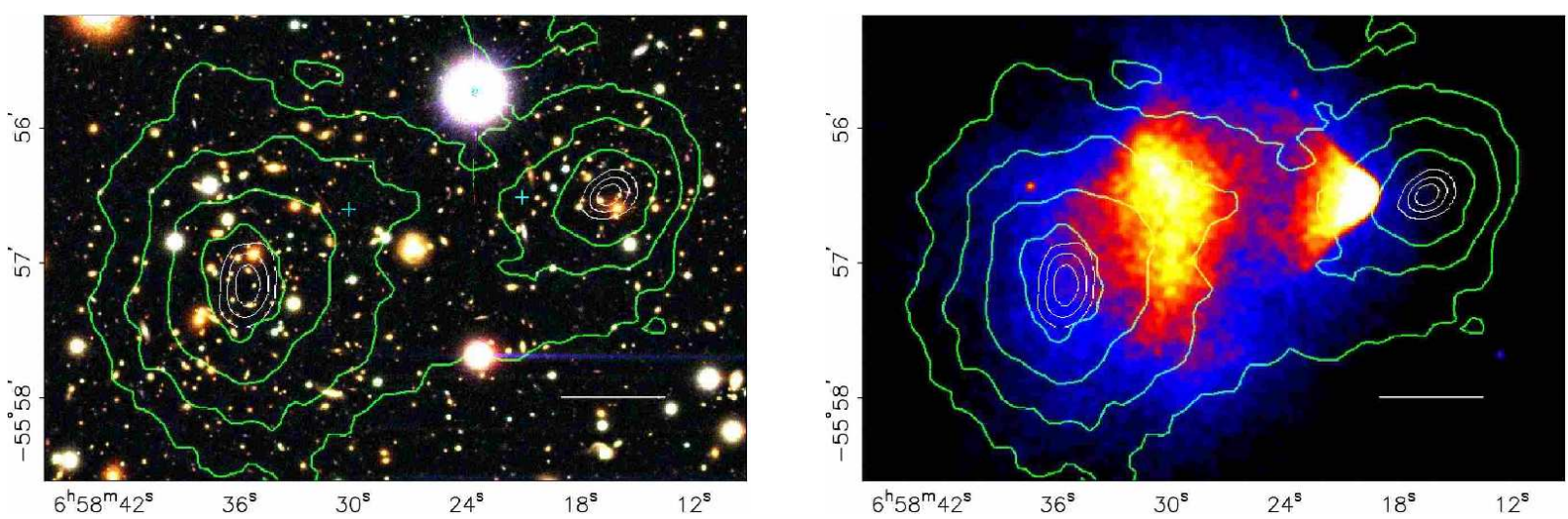

Figura 2.4: Imagens do aglomerado em fusão 1E0657-558 (Bullet Cluster) Esquerda:) no visual e

Direita:) em exposição de $500 \mathrm{ks}$ em raios-X . Os contornos verdes mostram a função $\kappa$, proporcional à densidade superficial de massa. O contorno mais externo representa $\kappa=0.16$ e os contornos internos foram calculados subtraindo-se $\Delta \kappa=0.07$. A barra branca no canto inferior tem $500 \mathrm{kpc}$ na posição do aglomerado. Figura extraída de Clowe et al. (2006).

do aglomerado, o gás intra-aglomerado sofre a pressão de arrasto (ram-pressure) sendo separado da componente estelar que, por sua vez, se comporta como um fluido acolisional.

Estudos baseados no efeito de lente gravitacional fraca mostraram que os centros de massa total de cada um dos aglomerados progenitores não acompanhavam o centro de massa de suas componentes plasmáticas (Clowe et al., 2006), conforme mostrado pelos mapas de potencial na figura 2.4. Como em aglomerados a principal contribuição bariônica à massa está na forma de gás, concluímos que deva existir uma componente adicional de massa não visível e acolisional, acompanhando o movimento da parte estelar. Estudos de sistemas com caraterísticas similares observados posteriormente corroboram essa hipótese (Bradač et al., 2008).

Além do que discutimos até este ponto, outras evidências independentes da existência da matéria escura vêm de, por exemplo, (i) vínculos provenientes da nucleossíntese primordial, (ii) medidas da temperatura e polarização das anisotropias da CMB, (iii) investigações das estruturas em largas escalas das oscilações acústicas dos bárions (BAO), (iv) limites cosmológicos ao parâmetro de densidade de matéria escura, obtidos de amostras de dados independentes entre si e (v) simulações numéricas de estruturas em grandes escalas (Roos, 2010).

Em particular, em um universo no qual toda sua matéria fosse bariônica, as perturbações de temperatura na CMB precisariam ser aproximadamente 100 vezes maiores do que o valor medido $\left(\delta T / T \approx 10^{-5}\right)$ para que houvesse a formação de estruturas no universo 
como as observamos (Komatsu et al., 2010; Planck Collaboration XVI: Ade, P. A. R. et al., 2013). Essa conclusão é derivada do fato de que, como a matéria bariônica permanece acoplada aos fótons até a recombinação, as perturbações de densidades nessa componente não podem se desenvolver até esse momento. Entretanto, a matéria não-bariônica começa a se aglomerar após a equipartição matéria-radiação, desenvolvendo as perturbações de densidade que originarão as estruturas observadas. A ausência de matéria escura leva a incoerências graves na teoria de formação de estruturas, como a não formação de galáxias, por exemplo.

As evidências observacionais e a teoria de formação de estruturas indicam que a matéria escura deva ser composta majoritariamente de partículas sem pressão. Enquanto a matéria escura relativística ou "quente" (Hot Dark Matter - HDM) apaga as flutuações de densidade em pequenas escalas na época da formação de estruturas, a matéria escura fria (Cold Dark Matter - CDM) se aglomera em pequenas escalas, formando poços de potencial para a matéria bariônica (Peacock, 1999). Esse esquema de formação de estruturas, das pequenas escalas para as maiores, está de acordo com o cenário bottom-up do modelo hierárquico e é o que melhor descreve as observações (White e Rees, 1978; Blumenthal et al., 1984).

Em suma, ainda que a deteç̧ão direta de partículas de matéria escura ainda não tenha ocorrido, as evidências de origens cosmológicas e astrofísicas indicam que essas partículas devam interagir fracamente entre si e com a matéria bariônica e ainda que emitam e absorvam radiação em taxas desprezíveis. Além disso, as evidências provenientes da nucleossíntese primordial, das observações da CMB e da teoria de formação de estruturas mostram que essa nova componente não pode ser formada de matéria bariônica como restos estelares ou halos massivos colapsados. No contexto do modelo padrão, a detecção de matéria escura não bariônica utilizando apenas dados da CMB é confirmada com $86 \sigma$ de confiança estatística (White e et al., 2014). Medidas de velocidade de rotação de alguns objetos indicam com $5 \sigma$ de confiança a existência de matéria escura mesmo dentro do raio da órbita solar na Galáxia (Iocco, F. and Pato, M. and Bertone, G., 2015). A matéria escura é, portanto, um ingrediente fundamental aos modelos cosmológicos capazes de explicar o universo observado. 


\subsubsection{Aceleração Cósmica}

Em 1998, uma das características mais surpreendentes e intrigantes do universo foi revelada. Dois grupos de pesquisa concluíram de forma independente que o universo passa por uma fase de expansão acelerada, através de observações da magnitude aparente de Supernovas do Tipo Ia (Riess et al., 1998; Perlmutter et al., 1999).

Utilizando dados de 42 supernovas com redshifts no intervalo [0.18, 0.83] aliados a uma subamostra com supernovas em baixos redshifts $(\lesssim 0.1)$, o grupo Supernova Cosmology Project concluiu que, para um modelo com constante cosmológica, matéria escura e matéria bariônica, a condição $\Omega_{\Lambda}=0$ estaria excluída com $99 \%$ de confiança estatística, independentemente da curvatura espacial do modelo (figura 2.5) . No caso plano, a análise estatística apontou como melhor ajuste $\Omega_{m}=0.28_{-0.12}^{+0.14}$ para $1 \sigma$ de confiança estatística, o que resulta em um parâmetro de desaceleracão $q_{0}=-0.58$, implicando em universo em fase de expansão acelerada (Perlmutter et al., 1999).

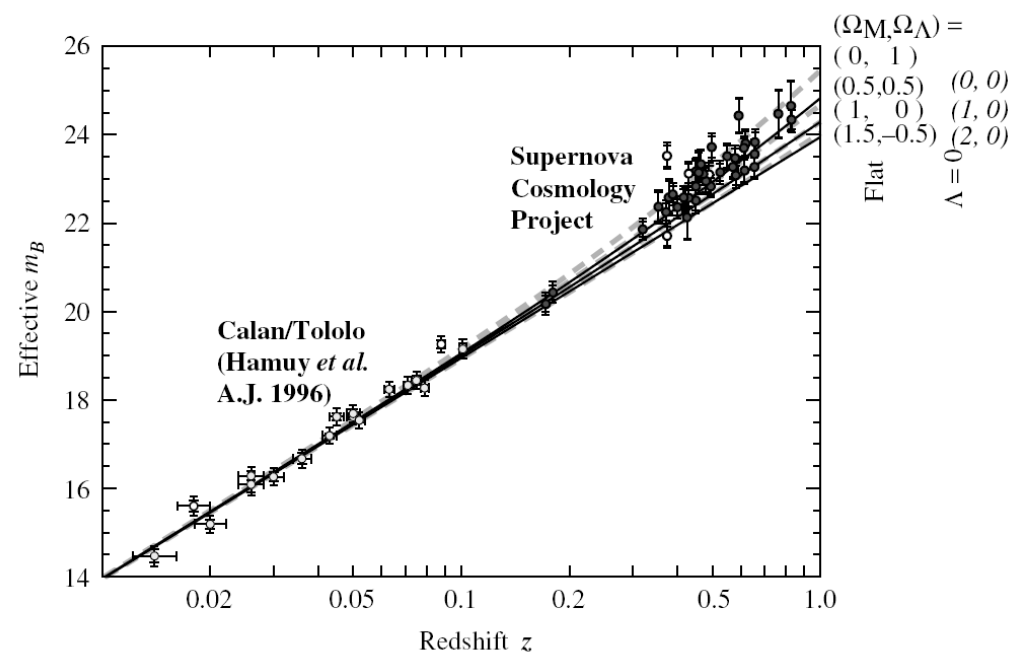

Figura 2.5: Diagrama de Hubble-Sandage contendo as 42 SNe Ia observadas pelo Supernova Cosmology Project (Perlmutter et al., 1999).

Paralelamente, o High-z Supernova Search Team utilizou uma amostra com 16 SNe Ia, com redshifts no intervalo [0.16,0.97] e 34 SNe Ia em baixos redshifts para chegar à conclusão de que, em um modelo com matéria escura e constante cosmológica, temos $\Omega_{\Lambda}>$ 0 com $99.7 \%$ de nível de confiança estatística, independentemente do valor do parâmetro de curvatura (figura 2.6). O melhor ajuste obtido no modelo plano foi de $\Omega_{m}=0.28 \pm 0.10$ e, assumindo $\Omega_{\Lambda}>0$, eles obtiveram $q_{0}<0$ com $95 \%$ de confiança estatística. 


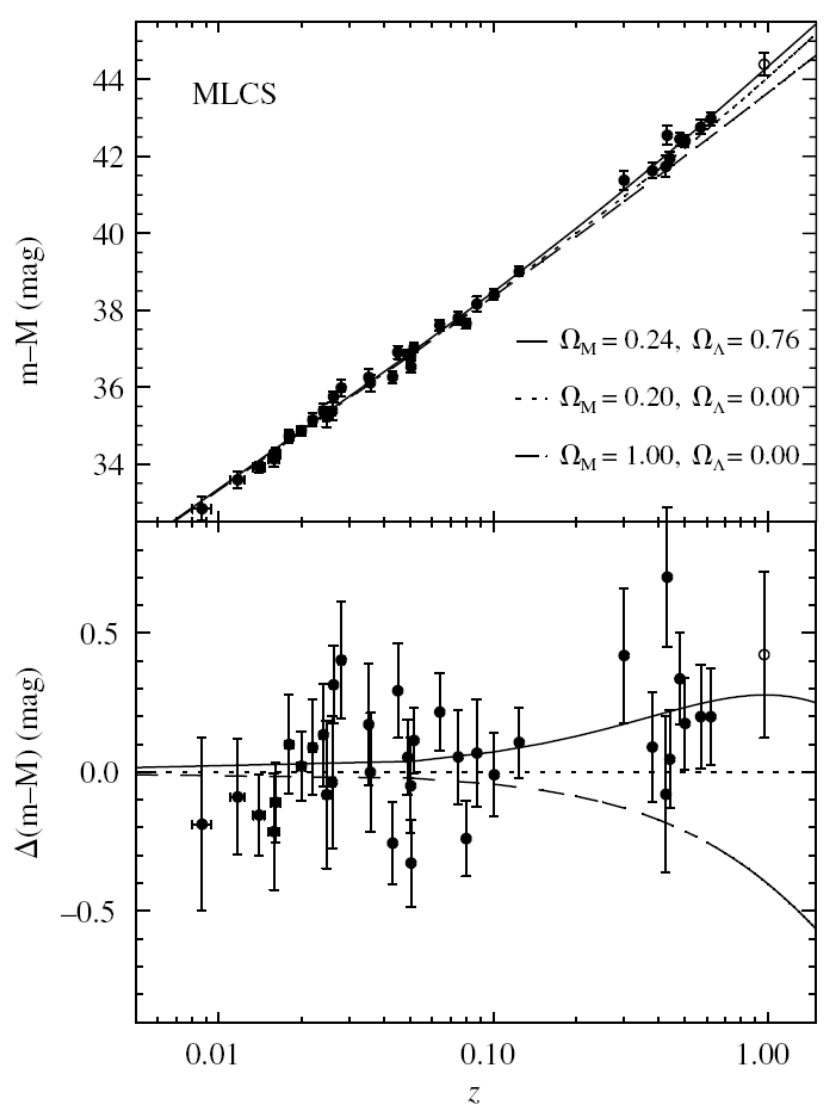

Figura 2.6: Diagrama de Hubble-Sandage das observações de supernovas do Tipo Ia contendo as $16 \mathrm{SNe}$ Ia observadas pelo High-z Supernova Search (Riess et al., 1998).

A conclusão acerca da aceleração cósmica está diretamente ligada às medidas de luminosidades aparente abaixo do esperado para um universo apenas com matéria. Entre as tentativas de explicar essa luminosidade aparente reduzida, a mais discutida foi a absorção e o espalhamento da luz proveniente da SN Ia pela hipotética poeira cinza (gray dust) (Aguirre, 1999; Drell et al., 2000). Contudo, caso a poeira cinza tivesse papel considerável nessas observações, esperar-se-ia que a redução da luminosidade aparente aumentasse com o redshift sempre. Entretanto, observações de SNe Ia em altos redshifts, como por exemplo da SN1997ff (Gilliland et al., 1999) em $z=1.7 \pm 1$, mostram que, se a poeira tivesse papel significante, seu efeito desapareceria exatamente quando é previsto que a energia escura começa a acelerar o universo.

O número cada vez maior de medidas de SNe Ia, com precisão crescente e melhor conhecimento sobre as fontes de erros sistemáticos vêm fortalecendo as evidências da aceleração cósmica (Frieman et al., 2008; Suzuki et al., 2012; Betoule et al., 2014). O estabelecimento 

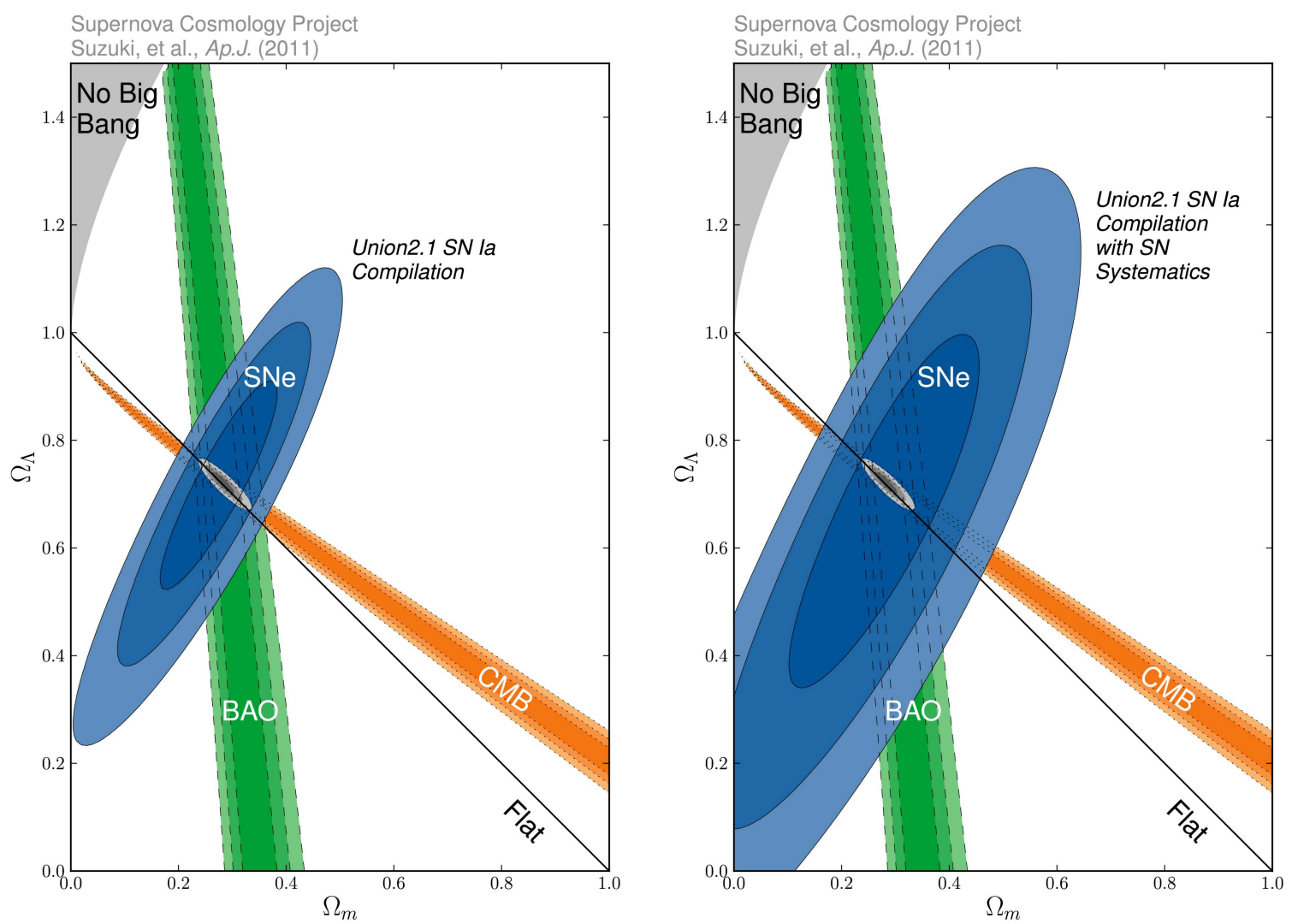

Figura 2.7: Vínculos obtidos sobre os parâmetros livres do modelo $\Lambda$ CDM. As regiões com 68.3\%, 95.4\% e $99.7 \%$ de nível de confiança estatística no plano $\Omega_{m}-\Omega_{\Lambda}$ delimitam os vínculos obtidos das análises de SNe Ia (azul), BAO (verde), CMB (laranja) e da combinação dos anteriores (cinza). Os vínculos obtidos considerando-se apenas os erros estatísticos na análise de SN Ia são mostrados do lado esquerdo. Ao lado direito, são mostrados as regiões de confiança obtidas considerando-se os erros estatísticos e sistemáticos em SN Ia. Figura retirada de (Suzuki et al., 2012).

dessas observações representou um marco histórico para a Cosmologia, laureando S. Perlmutter, A. G. Riess e B. P. Schmidt com o prêmio Nobel de 2011 "Pela descoberta da expansão acelerada do Universo através de observações de Supernovas distantes".

Entre as mais completas amostras disponíveis de magnitude $\times$ redshift , está a compilação do Supernova Cosmoloqu Group conhecida por Union 2.1. com 580 medidas (Suzuki et al.,2012) (Figura 2.7). Recentemente, Betoule et al. (2014) submeteu um trabalho com a compilação de 740 curvas de luz, cujos ajustes aos parâmetros cosmológicos são apresentados na tabela 2.1. Com o aumento de observações de SNe Ia, o maior desafio observacional atual é a diminuição dos erros sistemáticos nessas medidas. Medidas de curvas de luz em altos redshifts com melhor resolução, estudos relacionando o efeito do progenitor sobre a luminosidade, entre outros, deverão ser capazes fornecer medidas de distâncias mais precisas (Frieman et al., 2008). 


\subsubsection{A Consolidação de um Novo Paradigma}

Por compararem a taxa de expansão em baixos e altos redshift, as observações de SNe Ia são as principais evidências da aceleração cósmica. De forma complementar, medidas de formação de estruturas, CMB e estruturas em largas escalas fornecem evidências adicionais de que o universo esteja acelerando.

Logo, mais uma vez a constante cosmológica $\Lambda$ foi evocada para conciliar teoria e observações cosmológicas. Em tal contexto, surge o atual modelo padrão em cosmologia, $\Lambda$ CDM. Em termos gerais, o modelo $\Lambda$ CDM é baseado (i) na TRG como teoria de gravitação e no princípio cosmológico como hipótese fundamental; (ii) na existência do Big Bang e da inflação cósmica, suportados por diversas observações, (iii) e em um universo atualmente composto em densidade de energia por cerca de $70 \%$ de energia do vácuo $\rho_{\Lambda}=\frac{\Lambda}{8 \pi G}, 25 \%$ de matéria escura, $5 \%$ de matéria bariônica e uma fração muito pequena de radiação (Suzuki et al., 2012; Planck Collaboration XVI: Ade, P. A. R. et al., 2013).

Nesse modelo, parte expressiva do conteúdo energético do universo, representada pela constante $\Lambda$, teria um efeito gravitacional repulsivo, sendo responsável pela aceleração. Dentro dos modelos expansionistas de Friedmann, a aceleração está relacionada ao conteúdo energético por

$$
\frac{\ddot{a}}{a}=-\frac{4 \pi G}{3}\left(\rho+3 p-\frac{\Lambda}{4 \pi G}\right) .
$$

Ao assumirmos um termo cosmológico não nulo, a aceleração atual do universo é garantida desde que

$$
\Lambda>4 \pi G(\rho+3 p)
$$

O modelo padrão $\Lambda$ CDM possui características muito interessantes como a sua simplicidade e sua ótima capacidade de ajuste à maior parte das observações. Contudo, ele se sustenta no fato de que aproximadamente $95 \%$ do universo é composto de matéria exótica nunca observada, tendo na sua componente central, a constante cosmológica, graves problemas conceituais. Isso estimulou a formulação de modelos alternativos, haja vista que o termo de constante cosmológica não é a única explicação possível para a aceleração cósmica.

Para a constante cosmológica nula $\Lambda=0$ e um fluido usual com $\rho>0$ e $p \geq 0$, a equação (2.54) será tal que $\ddot{a}<0$, mostrando que o universo nesse caso seria desacelerado. 


\begin{tabular}{|cccccc|}
\hline \hline Dataset & $\Omega_{m}$ & $\Omega_{k}$ & $H_{0}$ & $\Omega_{b} h^{2}$ & $\chi^{2} /$ d.o.f. \\
\hline \hline Planck+WP+BAO+JLA & $0.305 \pm 0.010$ & $0.002 \pm 0.003$ & $68.34 \pm 1.03$ & $0.0221 \pm 0.0003$ & $684.1 / 738$ \\
Planck+WP+BAO & $0.306 \pm 0.010$ & $0.002 \pm 0.003$ & $68.25 \pm 1.06$ & $0.0221 \pm 0.0003$ & \\
Planck+WP+SDSS & $0.397 \pm 0.108$ & $-0.019 \pm 0.026$ & $59.93 \pm 8.17$ & $0.0221 \pm 0.0003$ & $350.7 / 369$ \\
Planck+WP+SDSS+SNLS & $0.309 \pm 0.046$ & $0.001 \pm 0.011$ & $67.94 \pm 5.15$ & $0.0221 \pm 0.0003$ & $577.9 / 608$ \\
Planck+WP+JLA & $0.292 \pm 0.037$ & $0.005 \pm 0.009$ & $69.85 \pm 4.44$ & $0.0221 \pm 0.0003$ & $682.9 / 735$ \\
Planck+WP+C11 & $0.244 \pm 0.047$ & $0.015 \pm 0.010$ & $76.48 \pm 7.36$ & $0.0221 \pm 0.0003$ & $395.1 / 468$ \\
\hline
\end{tabular}

Tabela 2.1 - Valores de melhor ajuste para os parâmetros do modelo $\Lambda$ CDM e variação em $68.7 \%$ de nível de confiança estatística. Limites obtidos por Betoule et al. (2014).

Entretanto, para qualquer fluido exótico com pressão negativa suficientemente grande, a aceleração do universo pode ser obtida. Assumindo a existência de um termo de energia escura com densidade $\rho_{d e}$ e pressão $p_{d e}$, o universo é acelerado desde que a condição

$$
p_{d e}<-\frac{1}{3}\left(\rho+\rho_{d e}+3 p\right)
$$

seja satisfeita.

No capítulo seguinte, discutiremos algumas característica do modelo padrão em cosmologia assim como algumas das muitas alternativas a energia escura presentes na literatura. 
Capítulo 3

\section{Modelos Acelerados}

Nas últimas décadas, as observações cosmológicas têm aumentado significativamente em número. Melhores equipamentos e técnicas são responsáveis por restringir com precisão inédita os parâmetros cosmológicos. Do ponto de vista teórico, a cosmologia também se beneficia dos progressos em astrofísica, astropartículas e física de partículas elementares.

Deste cenário emerge o modelo $\Lambda \mathrm{CDM}$ ou modelo de concordância cósmica que, como o próprio nome indica, é capaz de descrever grande parte das observações realizadas. O estabelecimento desse modelo dissolveu muitas das tensões existentes entre as observações e o antigo modelo padrão, o chamado modelo de Einstein-de Sitter. Paradoxalmente, o atual modelo padrão se sustenta no fato de que aproximadamente $95 \%$ do conteúdo energético do Universo são compostos de fluidos escuros desconhecidos, sendo o valor estimado da energia escura no universo apontado como "provavelmente a pior previsão teórica de toda a história da física"1! Isso nos leva à questão: até que ponto o nosso conhecimento sobre o Universo realmente evoluiu (Li et al., 2011; Lima, 2004)?

Neste capítulo, discutiremos alguns dos principais candidatos a energia escura. Introduziremos também as alternativas em que a presença de um fluido escuro é dispensável para a aceleração do universo, como no caso das teorias de gravitação modificadas ou, no contexto da Relatividade Geral, nos modelos com criação de matéria.

\subsection{A Constante Cosmológica $\Lambda$}

Entre os candidatos a energia escura, a constante cosmológica $\Lambda$ é o mais simples e também o mais antigo. Originalmente introduzida e posteriormente abandonada por

\footnotetext{
${ }^{1}$ Hobson et al. (2006).
} 
Einstein, essa constante já foi revivida por diversas ocasiões na literatura, sempre com o intuito de dissolver tensões entre os modelos e as observações cosmológicas de cada época.

Em 1917, Einstein iniciou a era da cosmologia moderna através da aplicação da recém criada Teoria da Relatividade Geral (TRG) ao universo como um todo. Com esse fim, Einstein abandonou a sua proposta original de equações de campo em favor de uma formulação mais geral, contendo um termo constante adicional.

Uma vez que o tensor de Einstein $G^{\mu \nu}$ deve satisfazer à identidade de Bianchi $\left(G^{\mu \nu} ;{ }_{\nu}=\right.$ 0) e levando em consideração que a derivada covariante do tensor métrico é $g^{\mu \nu}{ }_{\nu}=0$, existe uma liberdade em adicionar um termo proporcional a $g^{\mu \nu}$ à teoria, i.e., (Dadhich, 2004)

$$
G^{\mu \nu}+\Lambda g^{\mu \nu}=8 \pi G T^{\mu \nu}
$$

A constante cosmológica $\Lambda$ foi adicionada às equações de campo com o intuito de obter soluções de universo estático, de acordo com a crença geral na época. Sem esse termo, um modelo auto-gravitante constituído apenas por matéria não relativística e inicialmente estático colapsaria em virtude da atração gravitacional. Como ilustração, na geometria de Schwarzschild, esse termo adicional geraria uma força repulsiva que aumenta com a distância, representando um potencial constante repulsivo.

Com a descoberta da expansão do universo, a constante cosmológica fora rejeitada por Einstein, alegando suas inconsistências físicas e estéticas (Soares, 2005). Porém, muitos autores defendem que a TRG é enriquecida por sua formulação incluindo a constante cosmológica, ao mesmo tempo em que mantém a sua natureza de teoria covariante da gravitação. Assim, ao propor a constante, Einstein deixou de prever as soluções expansionistas do universo e, ao descartá-la, restringiu desnecessariamente a sua teoria gravitacional (North, 1965).

Desde então, a natureza e o papel da constante cosmológica vem sendo recorrente tema de discussão na literatura (Edwards. 1972: Hawking. 1984: Weinberg. 1989: Padmanabhan e Choudhury, 2003). O mais recente capítulo na história da constante cosmológica se consolidou com a descoberta da expansão cósmica acelerada, onde esse fenômeno seria causado pelo termo de gravidade repulsiva associado à constante $\Lambda$.

O modelo cosmológico, dentro do cenário de Big Bang, formado por matéria escura fria, matéria bariônica, radiação e constante cosmológica é chamado de modelo $\Lambda C D M$ ou 
ainda, devido à sua ótima capacidade de ajuste às observações, modelo de concordância cósmica. Os vínculos sobre os principais parâmetros livres desse modelo, obtidos através de observações da radiação cósmica de fundo, são apresentados na tabela 3.1.

\begin{tabular}{|c|cc|cc|cc|}
\hline \hline Parâmetro & \multicolumn{2}{|c|}{ Planck } & \multicolumn{2}{c|}{ Planck + Lensing } & \multicolumn{2}{c|}{ Planck +WP } \\
& best-fit & Limites em 1 $\sigma$ & best-fit & Limites em 1 $\sigma$ & best-fit & Limites em 1 $\sigma$ \\
\hline \hline$\Omega_{\mathrm{b}} h^{2}$ & 0.022068 & $0.02207 \pm 0.00033$ & 0.022242 & $0.02217 \pm 0.00033$ & 0.022032 & $0.02205 \pm 0.00028$ \\
$\Omega_{\mathrm{CDM}} h^{2}$ & 0.12029 & $0.1196 \pm 0.0031$ & 0.11805 & $0.1186 \pm 0.0031$ & 0.12038 & $0.1199 \pm 0.0027$ \\
$\tau$ & 0.0925 & $0.097 \pm 0.038$ & 0.0949 & $0.089 \pm 0.032$ & 0.0925 & $0.089_{-0.014}^{+0.012}$ \\
$\Omega_{\Lambda}$ & 0.6825 & $0.686 \pm 0.020$ & 0.6964 & $0.693 \pm 0.019$ & 0.6817 & $0.685_{-0.016}^{+0.018}$ \\
$\Omega_{\mathrm{m}}$ & 0.3175 & $0.314 \pm 0.020$ & 0.3036 & $0.307 \pm 0.019$ & 0.3183 & $0.315_{-0.018}^{+0.016}$ \\
$\sigma_{8}$ & 0.8344 & $0.834 \pm 0.027$ & 0.8285 & $0.823 \pm 0.018$ & 0.8347 & $0.829 \pm 0.012$ \\
$H_{0}$ & 67.11 & $67.4 \pm 1.4$ & 68.14 & $67.9 \pm 1.5$ & 67.04 & $67.3 \pm 1.2$ \\
$\Omega_{\mathrm{m}} h^{2}$ & 0.14300 & $0.1423 \pm 0.0029$ & 0.14094 & $0.1414 \pm 0.0029$ & 0.14305 & $0.1426 \pm 0.0025$ \\
$\Omega_{\mathrm{m}} h^{3}$ & 0.09597 & $0.09590 \pm 0.00059$ & 0.09603 & $0.09593 \pm 0.00058$ & 0.09591 & $0.09589 \pm 0.00057$ \\
Idade(Ganos) & 13.819 & $13.813 \pm 0.058$ & 13.784 & $13.796 \pm 0.058$ & 13.8242 & $13.817 \pm 0.048$ \\
\hline
\end{tabular}

Tabela 3.1 - Valores de melhor ajuste e limites em $1 \sigma$ de confiança estatística dos principais parâmetros do modelo $\Lambda$ CDM obtidos pelo satélite Planck (i) através das análises dos dados do espectro de potências de temperatura da CMB (colunas 2 e 3), (ii) da combinação entre os dados de temperatura e efeito de lentes gravitacionais (colunas 4 e 5) e (iii) da combinação entre os dados de temperatura com os dados de polarização da CMB em baixos multipolos do satélite WMAP (colunas 6 e 7). Tabela extraída de (Planck Collaboration XVI: Ade, P. A. R. et al., 2013).

O modelo $\Lambda$ CDM é um modelo fenomenologicamente simples, econômico e capaz de descrever diversas observações cosmológicas com considerável sucesso . Entretanto, mesmo fornecendo uma ótima parametrização ao universo observável, o modelo ainda possui questões cruciais a serem compreendidas. Entre as suas principais fraquezas ligadas à constante cosmológica, destacam-se o problema da constante cosmológica e o problema da coincidência cósmica (Weinberg, 1989; Hobson et al., 2006).

Nas equações de campo de Einstein, a constante cosmológica pode ser associada a uma densidade de energia:

$$
\rho_{\Lambda}=\frac{\Lambda}{8 \pi G}
$$

com pressão

$$
p_{\Lambda}=-\frac{\Lambda}{8 \pi G},
$$

ou seja, do ponto de vista dinâmico, o termo de constante cosmológica pode ser entendido como um fluido com equação de estado $p_{\Lambda}=-\rho_{\Lambda}$.

Por outro lado, a Teoria Quântica de Campos (TQC) associa o termo $\Lambda$ à densidade de energia do vácuo, i.e., às flutuações quânticas dos estados fundamentais dos campos 
no universo. Da invariância de Lorentz, o tensor de energia-momento para o vácuo é $T_{v a c}^{\mu \nu}=<\rho_{v a c}>g^{\mu \nu}$. Logo, a equação de estado para o vácuo é $p_{v a c}=-\rho_{v a c}$, exatamente como no caso da constante cosmológica (Zel'dovich, 1968). Nesse sentido, Bronstein (1933) originalmente propôs que o vácuo seria uma fonte de energia a ser considerada e associada à constante cosmológica (Kragh e Overduin, 2014).

Assim, um termo de energia do vácuo nas equações de campo de Einstein tem papel equivalente a adicionar à constante cosmológica um termo com a forma:

$$
\Lambda_{e f f}=\Lambda+8 \pi G \rho_{v a c}
$$

onde fica definido o valor efetivo da constante cosmológica como $\Lambda_{\text {eff }}$. De forma equivalente, podemos assumir uma energia do vácuo efetiva como:

$$
<\rho_{e f f}>=\rho_{<} v a c>+\frac{\Lambda}{8 \pi G} \equiv \frac{\Lambda_{e f f}}{8 \pi G} .
$$

Da TQC podemos obter estimativas teóricas da densidade energia do vácuo, enquanto as observações cosmológicas podem limitar os valores desse termo. Particularmente, as observações da CMB apontam que o universo deva ser plano e, portanto, a densidade de energia total deve ser, ao menos aproximadamente, igual à densidade de energia crítica do universo. Sabendo que o universo é composto também por matéria, temos assim um limite superior para o valor de densidade de energia do vácuo (Weinberg, 1989):

$$
\rho_{\Lambda} \lesssim \rho_{c 0} \sim \frac{H_{0}^{2}}{8 \pi G} \approx 10^{-47} G e V
$$

Em contrapartida, o vácuo de um campo quântico pode ser tratado como um conjunto infinito de osciladores quânticos independentes, cada qual contribuindo com suas próprias oscilações de ponto zero (Zel'dovich, 1968). Assim, cada modo de vibração de um dado campo de frequência $\omega$ adiciona à energia total o fator $\frac{1}{2} \hbar \omega$. Tomando um campo escalar de massa $m$, a contribuição de todas as energias de ponto zero de todos os modos normais até um número de onda limite $M \gg m$ é $(\hbar \equiv 1)$ :

$$
<\rho_{v a c}>=\frac{1}{2} \int_{0}^{M} \frac{4 \pi k^{2}}{(2 \pi)^{3}} \sqrt{k^{2}+m^{2}} d k \approx \frac{M^{4}}{16 \pi^{2}} .
$$

O número de onda $M$ está associado ao limite de energia em que o formalismo da TRG pode ser considerado confiável. Se tomarmos como limite a cromodinâmica quântica, o 
valor teórico $<\rho_{v a c}>$ ultrapassa em 42 ordens de grandeza o valor observacional $\rho_{\Lambda}$. Para a escala da teoria eletrofraca, esse valor é 54 ordens de grandeza maior que a estimativa observacional, enquanto nas escalas de energia de Planck, $<\rho_{v a c}>\sim 10^{74} \mathrm{GeV}$, levando a uma discrepância que ultrapassa 120 ordens de grandeza! Essa questão constitui o chamado problema da constante cosmológica sendo a maior discrepância obtida por uma teoria física.

Outra questão importante é conhecida como o problema da coincidência cósmica que consiste em entender a razão de o universo ter começado a se expandir de forma acelerada apenas recentemente ou, de modo equivalente, por que as densidades de matéria escura e de energia escura têm mesma ordem de magnitude apenas recentemente? Como ilustração desse problema, se a constante $\Lambda$ tivesse valor dez vezes maior do que o valor estimado, o universo teria iniciado sua aceleração muito antes, suprimindo a formação de estruturas cosmológicas como galáxias e aglomerados. Por outro lado, se $\Lambda$ tivesse um décimo do valor estimado, a aceleração cósmica não seria detectável.

Embora ainda não exista uma solução em primeiros princípios para o PCC e para o problema da coincidência, muitas alternativas vêm sendo propostas na literatura, tanto em cosmologia quanto nas teorias de quânticas de campo. Entre as propostas fenomenológicas para esse problema, temos os modelos em que a energia do vácuo varia com o tempo, modelos baseados em campos escalares, descrições unificadas de matéria e energia escura, entre outros, conforme discutiremos a seguir.

\subsection{Matéria X, Phantom \& CPL}

Uma generalização natural da constante cosmológica passa pela modificação da sua equação de estado original, $p_{\Lambda}=-\rho_{\Lambda}$, por uma equação de estado para um fluido exótico com a forma $p_{x}=\omega_{x} \rho_{x}$.

Os casos em que $\omega_{x}=$ constante são classificados como modelos XCDM padrão, sendo $-1 \leq \omega_{x}<0$, ou como modelos XCDM estendido ou phantom energy (energia fantasma), quando $\omega<-1$ (Caldwell. R. R., 2002).

Podemos ainda admitir a possibilidade de uma energia escura dinâmica, assumindo que o parâmetro da equação de estado não seja constante. Nos modelos $\omega(z) C D M$, as componentes de matéria e energia escura se conservam separadamente e a densidade de 
energia escura está sujeita à equação de conservação

$$
\dot{\rho}_{x}+3 H[1+\omega(z)] \rho_{x}=0
$$

sendo $\omega(z)$ uma função arbitrária. Genericamente, a evolução da densidade de energia pode ser definida em função de um parâmetro efetivo da equação de estado $\omega_{\text {eff }}$ :

$$
\rho_{x}=\rho_{x 0}(1+z)^{3\left[1+\omega_{e f f}(z)\right]}
$$

onde $\omega_{\text {eff }}$ é definido como:

$$
\omega_{e f f}(z)=-1+\frac{1}{\ln (1+z)} \int_{0}^{\ln (1+z)}\left[1+\omega\left(z^{\prime}\right)\right] d\left[\ln \left(1+z^{\prime}\right)\right] .
$$

Um exemplo de parametrização muito utilizada em testes observacionais sobre a dinâmica da energia escura é a chamada parametrização de Chevallier-Polarski-Linder (CPL), definida como (Chevallier e Polarski, 2001; Linder, 2003):

$$
\omega(a)=\omega_{0}+(1-a) \omega_{a}
$$

ou, em termos, do redshift

$$
\omega(z)=\omega_{0}+\omega_{a} \frac{z}{1+z},
$$

sendo $\omega_{a}$ e $\omega_{0}$ constantes do modelo.

Os modelos apresentados têm em comum o caso particular $\omega=-1$, sendo muito utilizados em testes observacionais que buscam desvios do modelo $\Lambda$ CDM. Para a parametrização CPL, a análise combinada de dados da radiação cósmica de fundo e oscilações acústica de bárions obteve como vínculos $\omega_{0}=-1.04_{-0.69}^{+0.72}$ e $\omega_{a}<1.32$, com $95 \%$ de nível de confiança estatística (Planck Collaboration XVI: Ade, P. A. R. et al., 2013).

No caso em que $\omega$ é constante, os vínculos obtidos da combinação de dados da anisotropia de CMB, medidas de polarização e medidas da constante de Hubble vinculam $\omega=-1.24_{-0.19}^{+0.18}$ com $95 \%$ de nível de confiança estatística, indicando uma tensão com o modelo $\Lambda$ CDM (Planck Collaboration XVI: Ade, P. A. R. et al., 2013). Isto é, os dados favorecem um modelo de energia fantasma em $2 \sigma$.

Embora muito discutidos na literatura, modelos de energia fantasma não possuem uma descrição teórica satisfatória (Caldwell et al., 1998, 2003). A versão mais simples desse modelo se motiva em um campo escalar com um termo cinético modificado (Chiba et al., 2000). Algumas versões encontradas na literatura também têm motivação em cosmologia de branas (McInnes, 2002; Sahni e Shtanov, 2003; Carroll et al., 2003). 


\subsection{Quintessência \& Campos Escalares}

O termo quintessência vem do Latim "quinto elemento", denotando um elemento além daqueles que formariam os elementos básicos do nosso mundo, segundo a filosofia grega. Em cosmologia, o termo quintessência, primeiramente utilizado por Caldwell et al. (1998), está associado à existência de um campo escalar remanescente $\phi$ com potencial $V(\phi)$, capaz de cumprir o papel de energia escura. Tal campo pode ser descrito por uma ação com a forma $(\hbar=1)$ :

$$
S=\int d^{4} x \sqrt{-g}\left[-\frac{1}{2} g^{\mu \nu} \partial^{\mu} \phi \partial_{\nu} \phi-V(\phi)\right],
$$

onde $g$ é o determinante do tensor métrico.

O tensor energia-momento da quintessência é definido como (Kolb e Turner, 1990):

$$
T_{\mu \nu}=\partial_{\mu} \phi \partial_{\nu} \phi-g_{\mu \nu}\left[\frac{1}{2} g^{\gamma \delta} \partial_{\gamma} \phi \partial_{\delta} \phi+V(\phi)\right] \text {. }
$$

No cenário FLRW, o tensor energia-momento do campo escalar $\phi$ espacialmente homogêneo tem a forma $T^{\mu}{ }_{\nu}=\operatorname{Diaqonal}\left(\rho_{\phi}, p_{\phi}, p_{\phi}, p_{\phi}\right)$, sendo a densidade de energia e a pressão definidas como (Kolb e Turner, 1990; Amendola e Tsujikawa, 2010):

$$
\begin{gathered}
\rho_{\phi}=T_{0}^{0}=\frac{1}{2} \dot{\phi}^{2}+V(\phi), \\
p_{\phi}=\frac{1}{3} T_{i}^{i}=\frac{1}{2} \dot{\phi}^{2}-V(\phi) .
\end{gathered}
$$

Por consequência, o parâmetro da equação de estado tem a forma:

$$
\omega_{\phi} \equiv \frac{p_{\phi}}{\rho_{\phi}}=\frac{\frac{1}{2} \dot{\phi}^{2}-V(\phi)}{\frac{1}{2} \dot{\phi}^{2}+V(\phi)},
$$

que, no caso mais geral, pode ser uma função do redshift z. Quando o campo varia lentamente $\left(\dot{\phi}^{2}<<V(\phi)\right)$, o parâmetro $\omega_{\phi} \sim-1$ e, portanto, o campo cumpre um papel semelhante ao da constante cosmológica. Contudo, essas duas formas de energia escura são fisicamente diferentes, sendo o campo escalar uma componente dinâmica enquanto a energia do vácuo uma constante.

Considerando-se o campo minimamente acoplado com a gravidade e a condição $T^{\mu \nu} ; \nu=$ 0 , o campo $\phi$ obedece à equação:

$$
\ddot{\phi}+3 H \dot{\phi}+V^{\prime}(\phi)=0
$$


onde cada ponto $\left({ }^{*}\right)$ denota uma derivada em relação ao tempo e a linha $\left({ }^{\prime}\right)$ representa uma derivada em relação ao campo $\phi$. A equação de Klein-Gordon acima também pode ser obtida através da variação da ação (3.13) em relação a $\phi$ (Amendola e Tsujikawa, 2010).

Modelos baseados em campos escalares foram originalmente propostos para descrever a expansão exponencial no início do universo, quando a densidade de energia do universo seria dominada pelo potencial $V(\phi)$. Durante o regime inflacionário, o universo se resfria como consequência da expansão adiabática. Entretanto, conforme exigido pela nucleossíntese primordial, o universo precisa ser reaquecido ao fim da inflação. O reaquecimento ocorre então às custas do decaimento do campo em partículas relativísticas, quando o campo $\phi$ oscila rapidamente. Esse processo é responsável por toda a alta entropia do universo e termina efetivamente quando $\rho_{\phi}$ assume valores extremamente baixos ou nulo.

No universo tardio, podemos supor também que a evolução recente de $\rho_{\phi}$ seja lenta. Caso a variação de $\rho_{\phi}$ seja mais lenta que a variação de densidade da matéria, fatalmente haverá um momento em que a densidade de energia do campo escalar será o termo dominante e o universo evoluirá como sendo dominado por uma constante cosmológica efetiva (Caldwell, 2000; Peebles e Ratra, 2003). Assumir a quintessência como responsável pela aceleração atual, suscita também a questão de se o presente estágio acelerado e a era da inflação são causados pelo mesmo campo $\phi$.

Um caso particularmente interessante foi proposto por Peebles e Ratra (2003). Nesse cenário, uma parte do campo $\phi$ (chamado de inflaton) é responsável pela inflação e está associada à entropia do fim do período inflacionário. A parte restante do inflaton, responsável pela aceleração atual do universo, decresce muito lentamente até o mínimo do potencial

$$
V(\phi)=\frac{M^{\alpha+4}}{\phi^{\alpha}}
$$

onde $M$ tem dimensões de massa. Na era da matéria, o parâmetro da equação de estado é constante e possui a forma:

$$
\omega_{\phi}=-\frac{2}{\alpha+2}
$$

onde o inflaton tem as propriedades da constante cosmológica para o caso $\alpha=0$. Quando a densidade de energia do campo escalar começa a ser importante para a expansão cosmológica, o parâmetro $\omega_{\phi}$ deixa de ser constante, se distinguindo da cosmologia $\Lambda$ CDM. 


\subsection{Gás de Chaplygin}

Proposto originalmente por Kamenshchik et al. (2001) e desenvolvido por Bilic et al. (2002) e Bento et al. (2002), nesse cenário entende-se que o setor escuro, i.e., matéria mais energia escura, seja descrito por um único fluido. No cenário do chamado gás de Chaplygin (gC) considera-se a introdução de um fluido exótico com equação de estado:

$$
p_{g C}=-\frac{A}{\rho^{\alpha}},
$$

onde $A$ e $\alpha$ são constantes positivas. $\mathrm{O}$ caso $\alpha=1$ é conhecido com gás de Chaplygin (gC) (Kamenshchik et al., 2001), enquanto o caso $\alpha \neq 1$ é chamado de gás de Chaplygin generalizado (gCg), proposto em Bento et al. (2002). No caso $\alpha=0$, o modelo $\Lambda$ CDM é recuperado.

A propriedade mais interessante desse cenário é a possibilidade de que o gC possa naturalmente interpolar entre o comportamento da matéria não-relativística e o de um termo de energia escura (Bilic et al., 2002; Bento et al., 2002).

Tomando a expressão (3.21) e a lei de conservação de energia, obtemos:

$$
\rho(t)=\left[A+\frac{B}{a^{3(1+\alpha)}}\right]^{1 /(1+\alpha)},
$$

levando ao parâmetro de equação de estado

$$
\omega_{g C}=\frac{-1}{1+\frac{B}{A} a^{-3(1+\alpha)}}
$$

onde B é uma constante de integração.

Das expressões acima, vemos que a densidade evolui como a matéria não-relativística para altos redshifts, $\rho \propto a^{-3}$ e passa a ser dominada por uma constante $\rho \propto A^{1 /(1+\alpha)}$, para baixos redshifts (Copeland et al., 2006).

Embora o gás de Chaplygin seja um candidato interessante, foi mostrado que o modelo perturbado possui dificuldades (Bilic et al., 2002; Bento et al., 2002; Amendola et al., 2003). Entre essas dificuldades, por exemplo, o efeito da supressão das perturbações e a mudança no comprimento de Jeans na fase acelerada do modelo afetam o espectro de potências da CMB, gerando previsões conflitantes com as observações (Carturan e Finelli, 2003) .

Uma das formas de contornar esse problema é através da decomposição do gás em um termo de matéria escura e outro de energia escura. Limites obtidos nesse caso sobre o 
modelo barotrópico de gCG perturbado vincularam $\alpha \lesssim 5.710^{-6}$ com $95 \%$ de confiança estatística, ou seja, um modelo completamente degenerado com o $\Lambda$ CDM . No caso em que matéria escura segue geodésicas, os limites obtidos foram $0.15<-\alpha<0.26$ com 95\% de nível de confiança (Wang et al., 2013). Outros vínculos em modelos de gC e gCG podem ser encontrados em Cunha e Santos (2004); Lima et al. (2008); Alcaniz e Lima (2005b); Lima et al. (2009); Alcaniz et al. (2003); Dev et al. (2003); Makler et al. (2003). Estudos de perturbações nesses modelos foram realizados por Fabris et al. (2002); Zimdahl e Fabris (2005); Fabris et al. (2010); Borges et al. (2013); Campos et al. (2013), no caso linear, enquanto a formacão de estruturas foi abordada por Fernandes et al. (2012): Del Popolo et al. (2013); Caramês et al. (2014), entre outros.

\subsection{Modelos com Decaimento do Vácuo}

Nos modelos com decaimento do vácuo, ou modelos $\Lambda(\mathrm{t}) \mathrm{CDM}$, considera-se que a energia do vácuo possa variar ao longo da história do universo (Bronstein, 1933; Özer e Taha, 1986; Abdel-Rahman, 1992; Beesham, 1993; Lima e Maia, 1993; Waga, 1993; Lima e Maia, 1994; Lima et al., 1996; Lima e Trodden, 1996; Overduin e Cooperstock, 1998). Nesses modelos, a variação da energia do vácuo está diretamente relacionada com a interação entre energia escura e outras componentes cósmica havendo, em geral, transferência de energia de $\Lambda(t)$ para um outro fluido. Em suma, nesse cenário, o valor da energia escura começa muito alto e diminui ao longo do tempo, sendo uma alternativa para a reconciliação entre as estimativas provenientes da física de partículas e o valor observacional estimado hoje. Nesse contexto, o baixo valor observado de $\Lambda$ seria decorrente do fato de o universo ser velho.

Em geral, podemos descrever este cenário como tendo dois fluidos. Primeiramente, o fluido de energia escura é dotado de equação de estado

$$
p_{v}=-\rho_{v} \equiv \frac{\Lambda(t)}{8 \pi G}
$$

Como nesse caso a energia do vácuo deve ser variável, a identidade de Bianchi aplicada às equações de campo de Einstein tem como consequência

$$
8 \pi G T_{\mu \nu} ; \mu=\partial_{\nu} \Lambda .
$$


Logo, para que a conservação de energia momento seja satisfeita, é necessário que o fluido de energia escura esteja em interação com outro fluido 2, ou seja,

$$
\dot{\rho}+3 H(\rho+p)=-\frac{\Lambda(t)}{8 \pi G}
$$

Nesse cenário, as equações de campo de Einstein são dadas por $(K=0)$ :

$$
8 \pi G \rho+\Lambda(t)=3 \frac{\dot{a}}{a}
$$

$\mathrm{e}$

$$
8 \pi G p-\Lambda(t)=-2 \frac{\ddot{a}}{a}-\frac{\dot{a}}{a} .
$$

O principal objetivo dessa classe de modelos é descrever como a energia escura conduz à inflação e/ou à aceleração atual ao mesmo tempo em que o valor efetivo de $\Lambda$ decresce. Entretanto, não é claro qual seria a forma funcional de $\Lambda$, devendo esta ser determinada fenomenologicamente. Na literatura, a densidade de energia é geralmente associada a uma função implícita do tempo como, por exemplo, uma função do fator de escala $a$, do parâmetro de Hubble $H$ ou ainda de uma combinação de ambos (Freese et al., 1987; Carvalho et al.. 1992: Waga, 1993: Lima e Maia, 1994: Lima e Trodden, 1996: Alcaniz e Lima, 2005a; Lima et al., 2013; Perico et al., 2013; Andrade-Oliveira, F. et al., 2014).

Portanto, essa classe de modelos é capaz de resolver o problema da constante cosmológica, da coincidência cósmica e fornecer a aceleração cósmica, porém contendo uma arbitrariedade na determinação da forma de $\Lambda(t)$. Assim, a comparação entre as diferentes propostas de $\Lambda$ é em geral realizada por meio de análises estatísticas de dados cosmológicos. Em um resultado recente, Salvatelli et al. (2014) mostraram através da análise combinada de observações de diferentes naturezas que um cenário sem interação estaria excluído com 99\% de confiança estatística em detrimento de um cenário com interação tardia entre matéria e energia do vácuo.

Em síntese, observações cosmológicas de diversas naturezas apoiam fortemente a existência de uma componente de energia escura, sendo o candidato mais aceito a constante cosmológica $\Lambda$. Por outro lado, da existência dessa componente exótica emergem questões profundas.

\footnotetext{
${ }^{2}$ Deve-se notar que a primeira proposta de $\Lambda$ variável por Bronstein (1933) considerava o caso de $\Lambda$ variável apenas "por generalidade". Por outro lado, essa proposta desconsiderava a conservação de energia e associava a contínua diminuição do valor de $\Lambda$ como uma forma de definir uma seta do tempo (Kragh e Overduin, 2014)
} 
As observações cosmológicas fornecem evidências convincentes de que vivemos em um universo cujo 95\% de sua densidade de energia é indetectável diretamente através da luz e de outras formas de radiação eletromagnética. Dessa enorme fatia, somente $\approx 27 \%$ se manifesta como galáxias, aglomerados e quase $\approx 68 \%$ tem a forma de uma componente de propriedades exóticas e (pelo menos muito aproximadamente) homogênea. A quantidade de candidatos teóricos presentes na literatura é medida do quão pouco sabemos sobre a natureza da energia escura. Há inclusive entre os cosmólogos aqueles que questionam inclusive a existência desse termo, defendendo que os efeitos observados sejam decorrentes de inomogeneidades locais ou mesmo de uma nova teoria de gravitação a ser descoberta. Há um consenso de que os avanços nesse área dependem do desenvolvimento de novas observações e experimentos, bem como de uma compreensão mais profunda da física fundamental.

$\mathrm{Na}$ atual fase da cosmologia é fundamental que avancemos buscando alternativas teóricas capazes de descrever o universo, por mais estranhas e exóticas que estas possam parecer. Portanto, constitui uma questão crucial discutir a possibilidade de aceleração cósmica sem o uso constante cosmológica ou até mesmo desconsiderando um termo de energia escura.

Podemos classificar os principais modelos alternativos em três possibilidades:

(I) Modelos que mantém a TRG, mas rompem com o princípio cosmológico, como nos cenários inomogêneos, como em (Ishak et al., 2008, 2011; Lemaître e MacCallum, 1997; Clarkson, 2012).

(II) Modelos que alteram a TRG, como nas teorias $f(R)$, onde a ação de EinsteinHilbert é modificada por uma função do escalar de Ricci:

$$
S=\frac{1}{16 \pi G} \int d^{4} x \sqrt{-g} f(R)
$$

Embora essa abordagem não considere o termo de energia escura, existe uma certa arbitrariedade na determinação da função $f(R)$, dificultando o estabelecimento de limites teóricos e observacionais (Santos et al., 2007; Pereira et al., 2009; Clifton et al., 2012)

(III) Modelos dentro da TRG e assumindo o princípio cosmológico. Nessa classe, são englobados o modelo padrão com constante cosmológica, os modelos $X C D M$, o modelo de gás de Chaplygin, entre outras alternativas interessantes.

Em particular, alguns candidatos a energia escura estão diretamente ligados ao processo de criação de partículas, fornecendo um método de testes em potencial no campo da física 
de partículas. Nos modelos $\Lambda(\mathrm{t}) \mathrm{CDM}$ com decaimento do vácuo em matéria escura e sua contrapartida em um campo escalar, a criação de partículas pode ocorrer pela transferência de energia no setor escuro conforme discutiremos no capítulo 8 (Andrade-Oliveira, F. et al., 2014). Nos chamados modelos com criação de matéria escura, ou modelos CCDM (Lima et al., 1991; Calvão et al., 1992; Lima e Germano, 1992; Lima et al., 1996, 2008, 2010, 2011; Jesus et al., 2011; Jesus e Andrade-Oliveira, F., 2015), o processo de criação de partículas às custas do campo gravitacional gera um termo de pressão negativa nas equações de campo de Einstein, havendo a possibilidade desse processo ser responsável pela aceleração cósmica, conforme discutiremos nos capítulos 4, 6 e 7. 
Capítulo 4

\section{Cosmologias com Criação de Matéria}

\subsection{Introdução}

A origem e a criação dos constituintes materiais do universo estão entre as questões mais interessantes em cosmologia, sendo tema de diversos trabalhos na literatura.

Nos anos 1970, Tryon (1973) e Fomin (1975) propuseram o surgimento do conteúdo material do universo através de uma flutuação quântica do vácuo, com duração restrita pela relação de incerteza de Heisenberg $\Delta t \times \Delta E \sim \hbar$. Como exigência física, todas as quantidades conservadas deveriam ter valor total nulo e, em particular, a energia total seria compensada pela própria energia negativa da gravidade.

Parker (1968) e Fulling et al. (1974) estudaram o mecanismo de criação de partículas do ponto de vista microscópico, dentro da teoria quântica de campos em espaço-tempo curvo (Birrell e Davies, 1982). Nesses trabalhos, foi demonstrado que alguns constituintes do universo poderiam ser quanticamente criados por um campo gravitacional variável de um universo em expansão. A questão também foi abordada por Zeldovich (1981), onde um mecanismo efetivo de criação de matéria foi sugerido com o intuito de obter um modelo cosmológico livre do problema da singularidade inicial.

Na descrição da criação de partículas no contexto cosmológico, a questão fundamental a ser entendida é como o fenômeno de criação pode ser incorporada nas equações de campo de Einstein. Do ponto de vista macroscópico, a primeira formulação auto consistente de mecanismos de criação foi proposta por Prigogine et al. (1989) que, dentro um formalismo de termodinâmica de sistemas abertos, reinterpretaram o tensor energia momento incorporando a criação irreversível de matéria às equacões de campo de Einstein através de um termo adicional de pressão (Prigogine et al., 1989; ; Fulling et al., 1974). 
Um passo importante na compreensão da criação de partículas no universo foi dado por Lima et al. (1991); Calvão et al. (1992) que discutiram o processo de criação de partículas gravitacionalmente induzido através da teoria relativística para processos irreversíveis que generaliza naturalmente a formulação proposta por Prigogine et al. (1989). Adicionalmente, nesta teoria mostrou-se que, apesar de a viscosidade volumar e criação de partículas cumprirem papéis semelhante dentro da dinâmica cosmológica, tais mecanismos são completamente distintos do ponto de vista termodinâmico (Lima e Germano, 1992).

A inclusão da criação de partículas nas equações de campo via um termo efetivo de pressão abriu novas possibilidades para aplicações cosmológicas. Nos modelos com criação de matéria escura, os chamados modelos CCDM (Creation of Cold Dark Matter), propôs-se uma nova classe de modelos em que a criação de matéria escura fria gera um termo de pressão negativa, sendo portanto um mecanismo de aceleração cósmica.

Do ponto de vista quântico, o formalismo para a criação de matéria em cosmologias CCDM ainda é um grande desafio. Nesse cenário, a taxa de criação é decorrente da expansão em background e, por outro lado, a taxa de expansão é diretamente afetada pela criação de partículas. Recentemente, Jesus e Pereira (2014) discutiram a criação quântica de matéria escura contabilizando esse efeito de backreaction na expansão universal. Os vínculos obtidos sobre a massa da partícula de matéria escura foram poucos restritivos, mas a metodologia discutida abre boas perspectivas para as cosmologias com criação de matéria escura. Outro resultado interessante é discutido por Hook (2014), onde é demonstrado que, em um fenômeno semelhante à radiação Hawking para buracos negros primordiais em um espaço-tempo em expansão, é possível haver a criação de bárions e matéria escura.

Neste capítulo, discutiremos aspectos dinâmicos e termodinâmicos de um universo em que o número total de partículas de matéria escura (Cold Dark Matter - CDM) pode variar com o tempo. A abordagem adotada é através da formulação covariante da termodinâmica de não-equilíbrio para a cosmologia, onde são consideradas simultaneamente a criação de partículas de CDM e uma fonte de entropia (Prigogine et al., 1989; Calvão et al., 1992). Na seção 4.2, discutimos a termodinâmica básica do formalismo. A incorporação do mecanismo de criação nas equações de Friedmann, resultado fundamental para as discussões nos próximos capítulos, são discutidos na seção 4.3 . 


\subsection{Termodinâmica da Criação de Partículas}

Os estados termodinâmicos de um fluido relativístico simples podem ser descritos através das variáveis macroscópicas vetor fluxo de partículas, $N^{\alpha}$, vetor fluxo de entropia, $S^{\alpha}$, e pelo tensor energia momento $T^{\alpha \beta}$. Nessa formulação, o tensor energia momento tem a forma (Lima et al., 1991; Calvão et al., 1992; Lima e Germano, 1992)

$$
T^{\alpha \beta}=(\rho+P) u^{\alpha} u^{\beta}-P g^{\alpha \beta},
$$

onde $\rho$ é a densidade de matéria e $P$ é a pressão dinâmica que pode ser decomposta em duas contribuições:

$$
P=p+p_{c}
$$

sendo $p$ a pressão de equilíbrio termostático e $p_{c}$ um termo de correção associado a eventuais processos dissipativos no fluido (viscosidade volumar ou criação de matéria).

Cada uma das variáveis macroscópicas mostradas está sujeita a leis de conservações específicas.O tensor energia-momento (4.1) deve satisfazer à lei de conservação:

$$
T^{\alpha \beta} ;_{\beta}=0 .
$$

O número de partículas $\mathrm{N}$ contido em um volume $\mathrm{V}$, varia de acordo com

$$
\dot{N} / N=n \dot{V} /(n V)=\dot{n} / n+\Theta \equiv \Gamma
$$

sendo n a densidade de partículas, $\Theta=\dot{V} / V$ a expansão do fluido e $\Gamma$ a taxa de criação $(\Gamma>0)$ ou destruição de partículas $(\Gamma<0)$, medida em unidade de $\left(\right.$ tempo $\left.^{-1}\right)$. A fim de trabalharmos com grandezas covariantes, definiremos o vetor fluxo de partículas por (Calvão et al., 1992; Lima et al., 1991):

$$
N^{\alpha}=n u^{\alpha}
$$

que satisfaz a:

$$
N^{\alpha} ; \alpha=\Gamma
$$

A equação (4.6) é a versão covariante da equação (4.4). Na métrica de FLRW, as equações (4.4) e (4.6), tomam a forma: 


$$
\dot{n}+3 H n=\Gamma n .
$$

Analogamente, o vetor fluxo de entropia é definido por:

$$
S^{\alpha}=n \sigma u^{\alpha}
$$

onde $\sigma$ é a entropia específica ou entropia por partícula. Considerando-se a segunda lei da termodinâmica, $d S=d(n \sigma V) \geq 0$, o vetor fluxo de entropia deve satisfazer à lei:

$$
S^{\alpha}{ }_{\alpha}=n \dot{\sigma}+3 H n \sigma \geq 0
$$

ou seja, a criação de partículas pode ser associada a um processo irreversível. Além disso, pode-se observar que termodinamicamente o processo apresentado é completamente distinto da viscosidade volumar, uma vez que, mesmo no caso adiabático $\dot{\sigma}=0$, o processo de criação ainda é capaz de aumentar a entropia do sistema (Lima e Germano, 1992).

\subsection{Dinâmica Cósmica e Criação de Matéria Escura}

Iniciamos por considerar o elemento de linha homogêneo e isotrópico da métrica de Friedmann-Lemaître-Robertson-Walker (FLRW)

$$
d s^{2}=d t^{2}-a^{2}(t)\left(\frac{d r^{2}}{1-K r^{2}}+r^{2} d \theta^{2}+r^{2} \sin ^{2} \theta d \phi^{2}\right) .
$$

Nesse contexto, as equações de Friedmann para um universo composto por bárions, matéria escura, radiação e com criação de partículas têm a forma:

$$
8 \pi G \rho=3 \frac{\dot{a}^{2}}{a^{2}}+3 \frac{K}{a^{2}},
$$

$\mathrm{e}$

$$
8 \pi G\left(p+p_{c}\right)=-2 \frac{\ddot{a}}{a}-\frac{\dot{a}^{2}}{a^{2}}-\frac{K}{a^{2}} .
$$

Da conservação do tensor energia momento, temos:

$$
\dot{\rho}+\Theta\left(\rho+p+p_{c}\right)=0 .
$$

Nesse sistema, a lei de Gibbs é dada por

$$
n T \sigma=d \rho-\frac{\rho+p}{n} d n,
$$


onde T é a temperatura. Combinando as relações acima, obtemos

$$
S^{\alpha}{ }_{\alpha}=n \sigma \Gamma+n \dot{\sigma}=-\frac{3 H p_{c}}{T}-\frac{\mu n \Gamma}{T} \geq 0,
$$

onde utilizamos que na métrica de FLRW, $\Theta=3 H$, além da relação de Euler, dada por

$$
\mu=\frac{\rho+p}{n}-T \sigma
$$

Seguindo a nomenclatura adotada na literatura, entendemos como o caso "adiabático" da criação de matéria gravitacional quando a entropia específica permanece constante $(\dot{\sigma}=0)$. Como consequência, a entropia total é aumentada pela criação de partículas, i.e.,

$$
S^{\alpha} ;_{\alpha}=n \sigma \Gamma \geq 0
$$

Dessa forma, a segunda lei da termodinâmica implica que a taxa de criação de partículas só possa ser positiva ou, como no caso de um fluido perfeito, nula.

Reescrevendo a relação (4.16), obtemos

$$
n \sigma=\frac{\rho+p}{T}-\frac{\mu n}{T}
$$

e, aplicando a relação de "adiabaticidade" na expressão (4.15), obtemos

$$
\frac{3 H p_{c}}{T}=-\left(n \sigma+\frac{\mu n}{T}\right) \Gamma
$$

Assim, no caso $(\dot{\sigma}=0)$, a pressão de criacão é dada por (Prigogine et al., 1989: Lima et al., 1991; Calvão et al., 1992)

$$
p_{c}=-\frac{\rho+p}{3 H} \Gamma
$$

Em relação à equação acima, devemos notar que, uma vez que a positividade de $\Gamma$ é assegurada pela inequação (4.17), a pressão de criação será sempre negativa desde que o fluido criado satisfaça à condição $p+\rho>0$. Portanto, para criação de partículas de um fluido usual, o processo apresentado aqui é uma potencial causadora da aceleradora cósmica.

O efeito da pressão de criação sobre a cosmologia pode ser visualizado diretamente através do comportamento do fator de escala. Por exemplo, consideremos o fenômeno de criação em um modelo de universo composto por um fluido com equação de estado $p=\omega \rho$. Através das equações (4.11) e (4.12) pode-se verificar que (Lima et al., 2007): 


$$
2 a \ddot{a}+\left[(1+3 \omega)-(1+\omega) \frac{\Gamma}{H}\right]\left(\dot{a}^{2}+K\right)=0,
$$

que, no caso particular $\Gamma=0$, se reduz à equação usual de evolução do fator de escala para um modelo composto por um único fluido perfeito .

A pressão de criação deixa o parâmetro de desaceleração (2.29) com a forma:

$$
q(z)=\frac{1}{2} \frac{8 \pi G \rho}{3 H^{2}}\left[1+3 \omega-(1+\omega) \frac{\Gamma}{H}\right]
$$

ou ainda, para o caso plano:

$$
q(z)=\frac{1}{2}\left[1+3 \omega-(1+\omega) \frac{\Gamma}{H}\right],
$$

Modelos atualmente acelerados devem satisfazer a condição $q(z=0)<0$. Para o caso com criação, podemos afirmar que um modelo é acelerado hoje se :

$$
\Gamma(z=0)>H_{0} \frac{(1+3 \omega)}{1+\omega} .
$$

A natureza do mecanismo de criação, onde a expansão cosmológica é afetada pelo processo de criação ao mesmo tempo em que a taxa de criação é determinada pela expansão, cria dificuldades na determinação da taxa $\Gamma$ a partir de primeiros princípios. Por outro lado, como $\Gamma$ afeta a dinâmica do universo, essa taxa pode ser fenomenologicamente inserida nos modelos cosmológicos. Portanto, assumindo esse processo como mecanismo responsável pela aceleração cósmica, é possível vincular a criação de matéria assim como determinar se a taxa de criação é capaz de ajustar as observações cosmológicas. Discutiremos nos próximos capítulos dois dos modelos cosmológicos baseados nesse fenômeno (Lima et al., 2010, 2011; Jesus et al., 2011; Jesus e Andrade-Oliveira, F., 2015). 
Capítulo 5

\section{Criação de Matéria: Modelo de Lima, Jesus \& Oliveira}

\subsection{Introdução}

Conforme discutimos nos capítulos anteriores, atualmente um grande número de observações de naturezas distintas vem acumulando evidências de que o Universo seja homogêneo e isotrópico em grandes escalas, acelerado e com secção espacial plana. O candidato mais simples para essa aceleração é a existência de uma constante cosmológica (ou energia do vácuo) cujo valor pode ser vinculado através de observações cosmológicas. Embora a expansão acelerada venha sendo corroborada por diversas observações, o mecanismo responsável por esse fenômeno ainda não é compreendido, abrindo espaço para a discussão de diversas propostas.

Neste capítulo propomos e investigamos uma nova classe de modelos cosmológicos, constituído apenas de matéria escura e bariônica e acelerados por consequência do mecanismo de criação de partículas às custas da expansão gravitacional, de acordo com o formalismo apresentado no capítulo anterior. Iniciamos o presente capítulo discutindo o cenário com criação de matéria escura (CCDM) no qual propomos o cenário conhecido na literatura por modelo de Lima, Jesus \& Oliveira (LJO) (Lima et al., 2010). Mostramos que o modelo LJO reproduz o comportamento previsto pelo modelo $\Lambda \mathrm{CDM}$ em nível de background. Consequentemente, sem a presença de um termo de energia escura, esse modelo é capaz de ajustar com qualidade boa parte das observações cosmológicas como, por exemplo, as observações de SN Ia, a idade total do universo e a idade de objetos antigos, entre outros.

O modelo de criação pertence a uma classe de modelos fenomenológicos em que se considera que a energia do vácuo é cancelada por algum mecanismo físico ainda não com- 
preendido. Assim, uma vantagem básica desse cenário vem do fato de que é muito mais aceitável que a energia do vácuo seja desprezível ou nula do que finamente ajustada com outro fluido independente, como no caso da matéria escura e energia escura (Hawking, 1993; Li et al., 2011).

Na seção [5.2, discutimos a dinâmica cosmológica básica do modelo CCDM, partindo da escolha da taxa de criação $\Gamma$. Estendemos os resultados da seção anterior para o caso da criação de matéria com pressão na seção 5.3. Os limites observacionais obtidos sobre os parâmetros livres do modelo são apresentados na seção 5.4. Na seção 5.5, discutimos os principais resultados obtidos no modelo LJO em background.

\subsection{Modelo de Lima, Jesus \& Oliveira (LJO)}

A questão fundamental nos cenários cosmológicos com criação de matéria é a determinação do taxa de criação $\Gamma$. Na literatura, Lima et al. (2008) e Steigman et al. (2009) discutiram uma proposta com $\Gamma \propto H$, na qual a taxa de criação não favoreceria nenhuma época em especial durante a evolução do universo. Lima et al. (2008) mostraram que o modelo proposto é capaz de explicar tanto as observações de SNe Ia quanto resolver o problema da idade. Entretanto. ao inserir a componente bariônica no modelo. Steigman et al. (2009) mostraram que existe uma tensão entre os vínculos em baixos e altos redshifts , desafiando a viabilidade desse modelo.

Se por um lado, nos modelos CCDM a aceleração pode ser obtida de forma simples, a dificuldade primordial de tal classe de modelos é entender como tais modelos planos $\left(\Omega_{d} m+\Omega_{b}=1\right)$ se comportam na aglomeração de estruturas, onde um grande número de observacões dessa natureza indicam que $\Omega_{d} m+\Omega_{h} \approx 0.3$ (Allen et al.. 2002: Lima et al., 2003b; Rapetti et al., 2008; Vikhlinin et al., 2009). Em outras palavras, é necessário entender como a taxa de criação pode afetar a densidade de matéria de forma que exista uma parâmetro de densidade de matéria efetivo, capaz de conciliar o modelo plano e as estimativas observacionais. Discutiremos como uma escolha da taxa de criação $\Gamma$ é capaz de contornar tais dificuldades.

Inicialmente, devemos observar que a aceleração é um fenômeno recente na história do Universo, isto é, durante a fase de dominância da radiação o fenômeno responsável pela aceleração deve ter sido inibido. Uma possibilidade interessante para a taxa de criação é, 
portanto, uma função inversamente proporcional à densidade de matéria escura.

Em Lima et al. (2010), propomos como taxa de criação:

$$
\Gamma=3 \alpha H \frac{\rho_{c 0}}{\rho}
$$

onde $\alpha$ é uma constante adimensional positiva e $\rho_{c 0}$ é a densidade crítica de matéria atualmente. Observando-se a equação 4.20, notamos que com essa escolha da taxa $\Gamma$ para a criação de matéria escura fria $\left(p_{d m}=0\right)$, a pressão de criação adquire a forma:

$$
p_{c}=-\alpha \rho_{c 0}
$$

A criação de matéria escura adiciona um novo um termo de fonte à equação de balanço de matéria escura fria, de modo que :

$$
\dot{\rho}_{d m}+3 H \rho_{d m}=\Gamma \rho_{d m} \equiv 3 \alpha \rho_{c 0} H,
$$

cuja integração resulta em:

$$
\rho_{d m}=\left(\rho_{d m 0}-\alpha \rho_{c 0}\right)\left(\frac{a_{0}}{a}\right)^{3}+\alpha \rho_{c 0}
$$

ou, em termos do redshift, $1+z=\left(a_{0} / a\right)$,

$$
\rho_{d m}=\left(\rho_{d m 0}-\alpha \rho_{c 0}\right)(1+z)^{3}+\alpha \rho_{c 0} .
$$

Por outro lado, o termo $\Gamma$ não afeta diretamente as equações de conservação de radiação e de bárions usuais. Portanto, temos que:

$$
\begin{aligned}
& \rho_{r}=\rho_{r 0}(1+z)^{4}, \\
& \rho_{b}=\rho_{b 0}(1+z)^{3} .
\end{aligned}
$$

O parâmetro de Hubble do modelo é diretamente obtido ao inserirmos as expressões (5.5), (5.6), (5.7) nas equações de Friedmann (4.11) e (4.12):

$$
\left(\frac{H}{H_{0}}\right)^{2}=\left(\Omega_{m}-\alpha\right)(1+z)^{3}+\alpha+\left(1-\Omega_{m}\right)(1+z)^{2},
$$

onde a componente de radiação foi desprezada e definimos o valor atual do parâmetro de densidade da matéria total $\Omega_{m}$, tal que: 


$$
\Omega_{m} \equiv \Omega_{b}+\Omega_{d m}
$$

onde $\Omega_{b}$ e $\Omega_{d m}$ são os parâmetros de densidade dos bárions e da matéria escura, respectivamente, e utilizamos a condição de normalização

$$
\Omega_{k}=1-\Omega_{m}
$$

Embora o modelo proposto possua apenas uma componente escura, a semelhança com o modelo $\Lambda$ CDM passa a ser evidente. Lembrando que o parâmetro de Hubble para o modelo $\Lambda$ CDM tem a forma:

$$
\left(\frac{H_{\Lambda C D M}}{H_{0}}\right)^{2}=\Omega_{m}(1+z)^{3}+\Omega_{\Lambda}+\left(1-\Omega_{m}-\Omega_{\Lambda}\right)(1+z)^{2},
$$

notamos que ambos os modelos possuem parâmetros de Hubble similares, com $\alpha$ tendo o papel dinâmico de $\Omega_{\Lambda}$ e o termo $\left(\Omega_{m}-\alpha\right)$ cumprindo o papel de $\Omega_{m}$ encontrado no modelo $\Lambda C D M$. A semelhança fica ainda mais clara ao inserirmos na expressão (5.8) o parâmetro de densidade efetivo da matéria $\Omega_{m e f f}$, definido como:

$$
\Omega_{m e f f} \equiv \Omega_{m}-\alpha
$$

As dinâmicas cósmicas obtidas nos modelos LJO e $\Lambda$ CDM podem ser comparadas ainda observando-se a evolução do fator de escala. Considerando a pressão de criação (5.2) na equação (4.12), obtemos para o modelo LJO:

$$
2 a \ddot{a}+\dot{a}^{2}+K-3 \alpha H_{0}^{2} a^{2}=0,
$$

que pode ser comparada com a expressão equivalente obtida no modelo $\Lambda$ CDM:

$$
2 a \ddot{a}+\dot{a}^{2}+K-\Lambda a^{2}=0 .
$$

As expressões (5.13) e (5.14) mostram que ambos os modelos fornecem a mesma história de expansão cósmica, a menos da identificação $\alpha=\Lambda / 3 H_{0}^{2} \equiv \Omega_{\Lambda}$, corroborando a comparação realizada com base nas formas dos respectivos parâmetros de Hubble. Haja vista o sucesso do modelo $\Lambda$ CDM em explicar a maior parte das observações, tal característica é desejável a um candidato viável a modelo cosmológico. 
Por outro lado, embora os modelos LJO e CCDM forneçam descrições equivalentes da evolução cósmica, devemos ressaltar que tais cosmologias se baseiam em hipóteses físicas completamente independentes e, portanto, potencialmente tais modelos possam ser diferenciados. seia no campo da cosmologia ou da física de partículas (Lima et al.., 2010: Jesus e Pereira, 2014). Em particular, a positividade do parâmetro de Hubble em altos redshifts exige que a constante $\alpha$ satisfaça a

$$
\alpha \leq \Omega_{m}
$$

condição sem análogo no modelo $\Lambda$ CDM. Entretanto, tal condição ainda implica que

$$
\Omega_{m e f f} \geq 0
$$

reforçando a equivalência entre $\Omega_{\text {meff }}$ encontrado no modelo LJO e o parâmetro de densidade de matéria do modelo $\Lambda \mathrm{CDM}$.

Se considerarmos a secção espacial do Universo plana, conforme indicado pelas observações das anisotropias da CMB e previsto pelo modelo inflacionário, a equação (5.8) se reduz a

$$
H^{2}(z)=H_{0}^{2}\left[(1-\alpha)(1+z)^{3}+\alpha\right]
$$

onde $\Omega_{m}=1$. Neste caso, $\Omega_{m e f f}=1-\alpha$ e $\alpha$ e $H_{0}$ passam a ser os únicos parâmetros livres do modelo, exatamente como no caso $\Lambda \mathrm{CDM}$.

\subsection{Criação de Matéria Escura com Pressão no Cenário LJO}

Até o momento, analisamos a criação de partículas de matéria escura fria e as suas consequências cosmológicas. Ainda considerando a taxa de criação escolhida para o modelo LJO (5.1), vamos investigar como a criação de matéria escura com pressão afeta o cenário mostrado na seção 5.2 .

Ao admitirmos que a matéria escura possa ter pressão não nula, a evolução da densidade de matéria escura com pressão pode ser calculada através de:

$$
\dot{\rho}_{m}+3 H\left(\rho_{m}+p_{m}+p_{c}\right)=0
$$

onde a pressão da matéria escura é dada por 


$$
p_{m}=\varepsilon \rho_{m}
$$

sendo $\varepsilon$ a constante da equação de estado da matéria escura e $p_{c}$ a pressão de criação, cuja forma pode ser calculada através da expressão (4.20):

$$
p_{c}=-(1+\varepsilon) \alpha \rho_{c 0},
$$

para a taxa de criação em (5.1).

Substituindo (5.19) e (5.20) em (5.18), obtemos a evolução da densidade de matéria escura:

$$
\rho_{m}(z)=\left(\rho_{m 0}-\alpha \rho_{c 0}\right)(1+z)^{3(1+\varepsilon)}+\alpha \rho_{c 0}
$$

O parâmetro de Hubble pode ser obtido diretamente através da equação (4.11):

$$
H(z)=H_{0}\left[\left(\Omega_{m}-\alpha\right)(1+z)^{3(1+\varepsilon)}+\Omega_{b}(1+z)^{3}+\alpha+\left(1-\Omega_{m}-\Omega_{b}\right)(1+z)^{2}\right]^{1 / 2},
$$

ou, no caso plano $\left(\Omega_{m}=1\right)$

$$
H(z)=H_{0}\left[(1-\alpha)(1+z)^{3(1+\varepsilon)}+\Omega_{b}(1+z)^{3}+\alpha\right]^{1 / 2},
$$

\subsection{Vínculos Observacionais no Modelo LJO}

Discutimos aqui os vínculos obtidos sobre os parâmetros livres do modelo LJO, nos cenários com curvatura e plano. Para a análise estatística, utilizamos o teste de $\chi^{2}$ para a amostra de dados de Supernovas do tipo Ia do catálogo Union Compilation Data 2.1, com 580 dados observacionais de magnitude aparente corrigida por redshift (Suzuki et al., 2012).

Marginalizando analiticamente sobre a constante de Hubble $H_{0}$, o modelo com curvatura possui dois parâmetros livres a ser vinculados, a saber, o parâmetro de criação $\alpha$ e o parâmetro de densidade da matéria $\Omega_{m}$ ou, analogamente, o parâmetro de densidade efetiva da matéria $\Omega_{e f f}$. No caso plano, $\Omega_{m}=1$, resta como único parâmetro livre a constante $\alpha$. 

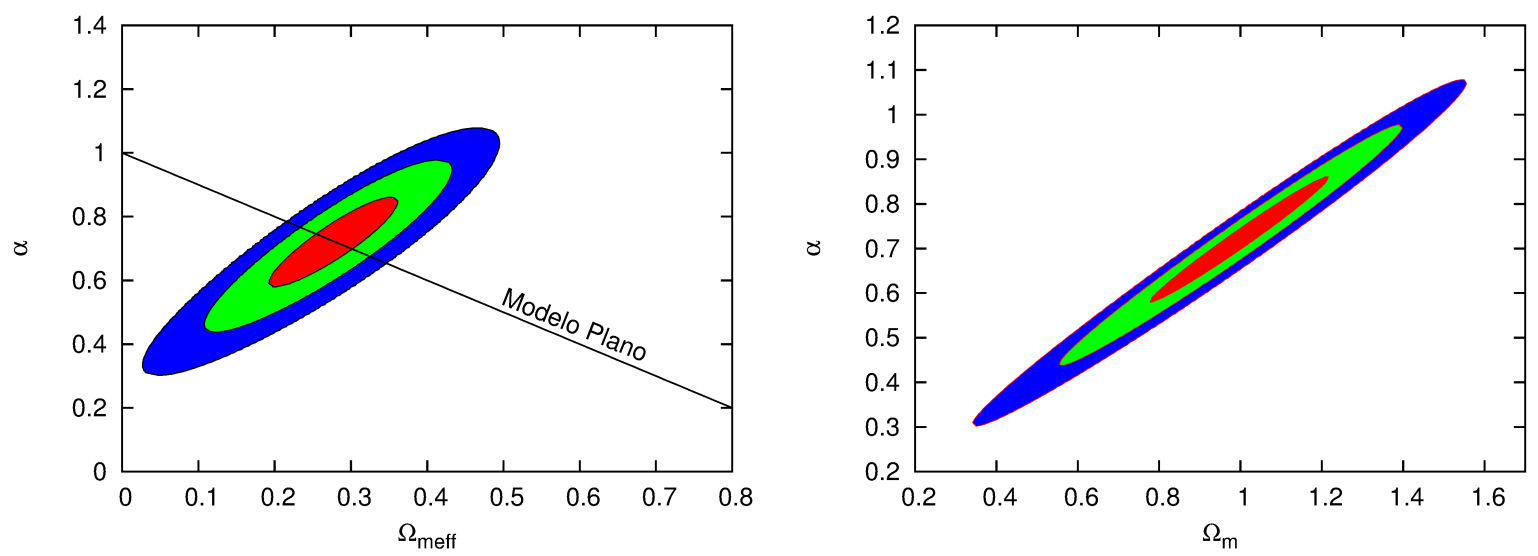

Figura 5.1: Esquerda:) Plano $\Omega_{m}-\alpha$ para o modelo LJO com curvatura, obtido através da análise de 580 dados de SN Ia do catálogo Union 2.1 (Suzuki et al., 2012). As regiões delimitam os níveis de confiança estatística de $68.3 \%, 95.4 \%$ e $99.7 \%$ e a linha preta passa pelos pontos no plano em que a condição de planura é satisfeita. Direita:) Níveis de confiança no plano $\Omega_{m}-\alpha$ para $68.3 \%, 95.4 \%$ e $99.7 \%$ de confiança estatística. Note que neste plano, o caso plano equivale a $\Omega_{m}=1$.

O módulo de distância previsto para uma supernova com redshift $\mathrm{z}$, dado o conjunto de parâmetros s, é dado por :

$$
\mu_{p}(z \mid \mathbf{s})=m-M=5 \log D_{L}+25,
$$

onde $m$ e $M$ são respectivamente, as magnitudes aparente e absoluta, o conjunto de parâmetros $\mathbf{s}=\left(H_{0}, \alpha, \Omega_{m}\right)$, no caso geral, ou $\mathbf{s}=\left(H_{0}, \alpha\right)$, no caso plano e $D_{L}$ é a distância de luminosidade (em unidades de $M p c$ ), calculada por:

$$
D_{L}=c(1+z) \int_{0}^{z} \frac{d z^{\prime}}{H\left(z^{\prime} \mid \mathbf{s}\right)}
$$

sendo $z^{\prime}$ uma variável de integração e $H(z \mid \mathbf{s})$ dado pela equação (5.8).

O conjunto de parâmetros s que fornece o melhor ajuste aos dados é encontrado utilizando uma estatística de $\chi^{2}$

$$
\chi^{2}=\sum_{i=1}^{N} \frac{\left[\mu_{p}\left(z_{i} \mid \mathbf{s}\right)-\mu_{o}^{i}(z)\right]^{2}}{\sigma_{i}^{2}},
$$

onde $\mu_{p}\left(z_{i} \mid \mathbf{s}\right)$ é dado por (5.24),$\mu_{o}^{i}(z)$ é o módulo de distância corrigido de uma SN Ia com $z_{i}, \sigma_{i}$ é a incerteza individual do módulo de distância observado e $N$ é o número de dados da amostra.

No lado esquerdo da figura 5.1, são mostradas as regiões de confiança no plano $\Omega_{m e f f^{-}}$ $\alpha$ obtidas no modelo LJO com curvatura para $68.3 \%, 95.4 \%$ e $99.7 \%$ de confiança estatística. Na análise conjunta com a marginalização analítica do parâmetro $h\left(H_{0}=\right.$ 
$\left.h 100 \mathrm{~km} \mathrm{~s}^{-1} M p c^{-1}\right)$, obtivemos $\alpha=0.725_{-0.145-0.287-0.415}^{+0.130+0.240+0.315}$ e $\Omega_{\text {meff }}=0.279_{-0.081-0.161-0.248}^{+0.079+0.152+0.215}$. No lado direito da figura [5.1, mostramos o resultado da análise estatística no plano $\Omega_{m}-\alpha$. Os limites obtidos em $68.3 \%, 95.4 \%$ e $99.7 \%$ de confiança estatística para os parâmetros livres foram $\Omega_{m}=1.004_{-0.227-0.446-0.659}^{+0.211+0.390+0.552}$ e $\alpha=0.725_{-0.145-0.287-0.415}^{+0.130+0.240+0.315}$. O valor mínimo do $\chi^{2}$ encontrado foi $\chi_{\min }^{2}=562.226$. Para os $\nu=578$ graus de liberdade, o valor reduzido $\chi^{2}$ obtido é de $\chi_{\nu}^{2} \approx 0.97$, mostrando que o modelo fornece um bom ajuste aos dados.

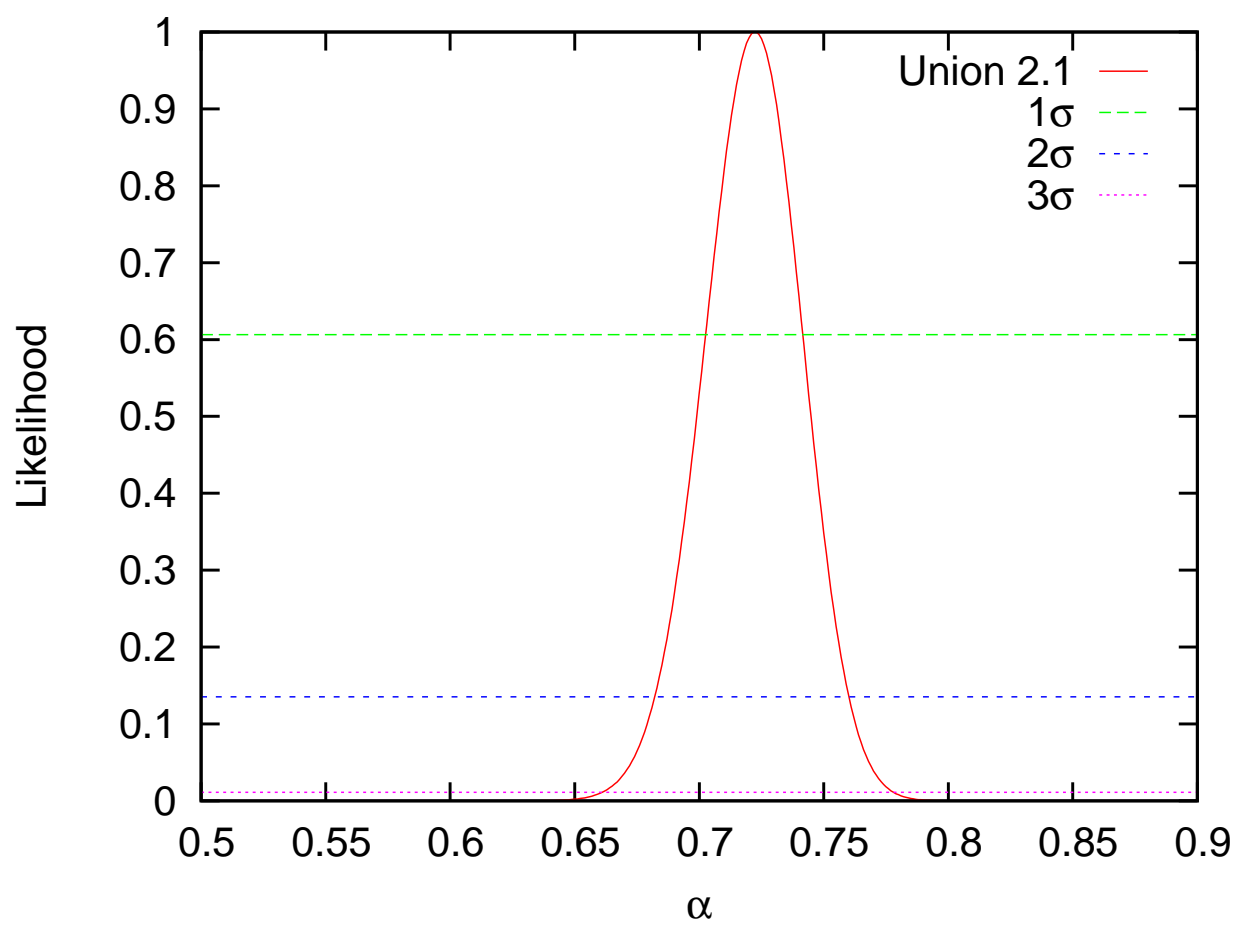

Figura 5.2: Vínculos obtidos sobre o parâmetro $\alpha$ no caso plano.

No caso plano, o modelo LJO tem $\alpha$ como único parâmetro livre. O vínculo encontrado utilizando a amostra Union 2.1 foi $\alpha=0.722_{-0.020}^{+0.020+0.039+0.056}$, para $68.3 \%, 95.4 \%$ e $99.7 \%$ de confiança estatística, respectivamente, com $\chi_{\min }^{2}=562.227$ e $\nu=579$ graus de liberdade. O valor encontrado para $\chi_{\min }^{2}$ reduzido, $\chi_{\nu}^{2}=\chi_{\min }^{2} / \nu$, foi $\chi_{\nu}^{2} \approx 0.97$.

Adicionalmente, realizamos uma análise estatística para o do modelo com criação de matéria escura com pressão no caso plano, a fim de estabelecer limites aos parâmetros livres $\alpha$ e $\varepsilon$. Admitimos como prior para o parâmetro $\varepsilon$ a variação entre os extremos $\varepsilon \geq-1$ (matéria com comportamento de constante cosmológica) e $\varepsilon \leq 1 / 3$ (matéria relativística). Além disso, utilizamos comoprior para a densidade de bárions $\Omega_{b} h^{2}=0.02229$ pois nesse caso, a matéria escura e os bárions estão dissociados por suas equações de estado. Através da análise estatística utilizando dados de SN Ia, obtivemos $\alpha=0.723_{-0.319}^{+0.111}$ e $\varepsilon=0.001_{-0.422}^{+0.333}$ 


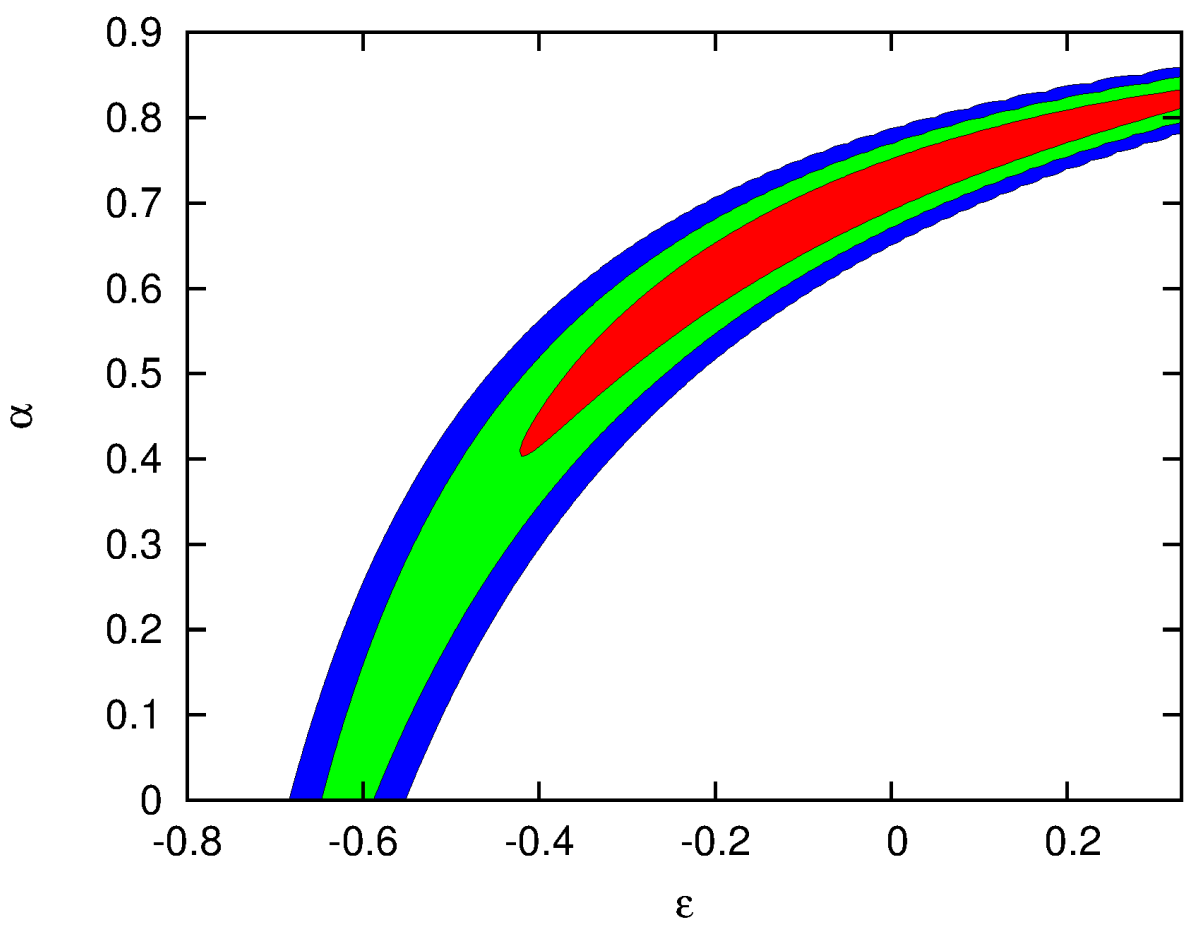

Figura 5.3: Vínculos sobre os parâmetros livres do modelo LJO plano com criação de matéria com pressão em $1 \sigma, 2 \sigma$ e $3 \sigma$ de nível de confiança. Os limites foram estimados considerando a amostra de dados de SN Ia Union 2.1. Os valores de melhor ajuste encontrados $\alpha=0.723_{-0.319}^{+0.111}$ e $\varepsilon=0.001_{-0.422}^{+0.333}$ para $1 \sigma$ com $\chi_{\min }^{2}=562.2$ e valor reduzido de $\chi_{\nu}^{2}=0.97$.

para $68.3 \%$ de confiança estatística. Para os valores de melhor ajuste, obtivemos $\chi_{\min }^{2}=$ 562.23. Nesse modelo, a constante da equação de estado da matéria escura acrescenta um parâmetro livre a mais em relação ao modelo plano LJO. O valor do $\chi^{2}$ reduzido, $\chi_{\min }^{2} / \nu \approx 0.97$, representando um ótimo ajuste aos dados 5.4. Entretanto, claramente os vínculos obtidos foram muito fracos.

\subsection{Discussões}

Neste capítulo discutimos uma proposta original de um modelo com criação de matéria escura (CCDM). Neste modelo é assumido que $\Omega_{\Lambda}=0$, contornando o problema da constante cosmológica. A aceleração tardia do universo é neste contexto atribuída ao processo irreversível de criação de matéria escura. Também se deve ressaltar que nesse modelo dominado por matéria escura não é necessário que o valor da constante de Hubble $H_{0}$ seja pequeno $\left(H_{0} \sim 50 \mathrm{~km} \mathrm{~s}^{-1} \mathrm{Mpc}^{-1}\right)$ para que o problema da idade seja resolvido. Entretanto, no arcabouço teórico do modelo existe a substituição da hipótese da existência de uma densidade de energia nunca observada diretamente (energia escura) por um meca- 


\begin{tabular}{|cc|}
\hline \hline$\Lambda$ CDM & LJO \\
\hline \hline$\Omega_{\Lambda}$ & $\alpha$ \\
$\Omega_{m}$ & $\Omega_{m e f f} \equiv \Omega_{m}-\alpha$ \\
$p_{\Lambda}=-\rho_{\Lambda}$ & $p_{c}=-\alpha \rho_{c 0}$ \\
Constante Cosmológica & Criação de Matéria \\
\hline
\end{tabular}

Tabela 5.1 - Comparação entre os modelos $\Lambda$ CDM e LJO

nismo fenomenológico ainda não bem compreendido (criação cosmológica de matéria).

O modelo discutido em particular é caracterizado pela taxa de criação fenomenológica $\Gamma=\alpha \frac{3 H}{\rho}$, sendo identificado na literatura como modelo LJO (Lima et al., 2010). É importante ressaltar que há uma equivalência dinâmica entre os modelos LJO e $\Lambda$ CDM em nível de background. A propósito, o modelo pode ser formalmente interpretado como um fluido com a mistura de duas componentes, a saber, (i) um termo de matéria escura sem pressão com parâmetro de densidade $\Omega_{m}-\alpha$ e (ii) um termo de densidade $\rho$ e pressão $p=-\alpha \rho_{c 0}$ que cumpre o mesmo papel dinâmico que a energia do vácuo (tabela [5.1). O modelo LJO com criação de matéria escura fria possui como parâmetros livres $\alpha$ e $\Omega_{m}$, no caso com curvatura, e no caso plano $\left(\Omega_{m}=1\right)$ apenas $\alpha$. Logo, os modelos $\Lambda$ CDM e LJO têm sempre o mesmo número de parâmetros livres.

No caso em que há criação de matéria "morna", é acrescentado um parâmetro adicional $\varepsilon$, ligado à pressão da matéria. Neste caso, o modelo passa a ter o mesmo número de graus de liberdade que um modelo constituído por $\Lambda$ e matéria escura com equação de estado $p=\varepsilon \rho$. Essa equivalência formal explica o ótimo ajuste que o modelo é capaz de dar às observações de SN Ia.

O mecanismo de criação adotado aqui, entretanto não possui uma formulação microscópica bem estabelecida, embora propostas interessantes venham sendo discutidas na literatura tanto no formalismo cinético relativístico quanto no formalismo em teoria quântica de campos (Zimdahl e Pavón, 1993; Zimdahl et al., 2001; Jesus e Pereira, 2014). Ainda assim, o formalismo básico é bem estabelecido, sendo o ponto crucial a determinação de como incorporar ao modelo quântico a criação de entropia e a taxa de criação associada aos modelos CCDM (Birrell e Davies, 1982; Grib e Dorofeev, 1994; Parker e Raval, 2001; Parker e Vanzella, 2004).

Em comparação com o modelo padrão adotado em cosmologia, ressaltamos que o modelo LJO possui dinâmica equivalente além de ser um modelo simples e ao mesmo tempo 
baseado na matéria escura, cujo status é relativamente superior ao da energia do vácuo. No próximo capítulo, discutiremos o desempenho do modelo em relação aos testes perturbativos. 
Capítulo 6

\section{Perturbações Lineares no Modelo de Lima, Jesus \& Oliveira}

\subsection{Introdução}

Avançamos em nossas discussões acerca do modelo LJO, analisando o modelo no regime de perturbações lineares. Para isso, desenvolvemos as perturbações através da abordagem Neo-Newtoniana, de forma que o papel da energia escura seja considerado mesmo em um uma abordagem não relativística. Os resultados obtidos foram comparados com dados da taxa de crescimento, gerando vínculos para o modelo perturbativo.

No presente capítulo, discutimos o formalismo Neo-Newtoniano e a sua validade na seção 6.2. Na seção 6.3, obtemos as perturbações em modelos CCDM com taxas de criação e velocidade efetiva do som genéricas. Esses resultados foram aplicados para o modelo LJO, na seção 6.4. As análises observacionais e a obtenção da função de crescimento de perturbações foram obtidas na seção 6.5. A validade da nossa análise e as discussões sobre o comportamento perturbativo do modelo LJO são sumarizados na seção 6.6.

\subsection{Formulação Neo-Newtoniana para Cosmologias com Criação de Matéria}

Conforme discutimos no capítulo (5), a dinâmica relativística não perturbada de cosmologias CCDM é equivalente à de um fluido simples com densidade $\rho$ e pressão $p_{c}$, conforme as expressões (4.11), (4.12). Além disso, caso o fluido equivalente possua o parâmetro da equação de estado $\omega=p / \rho$, da formulação de $p_{c}$ fica claro que em geral o parâmetro de equação de estado $\omega=-\frac{\Gamma}{3 H}$ pode variar com o tempo. 
Nesse sentido, é interessante considerar a abordagem quase-newtoniana para fluidos com pressão não nula proposta por Lima et al. (1997). Seguindo as abordagens desenvolvidas por McCrea (1951) e por Harrison (1965), tais autores propuseram a chamada descrição Neo-Newtoniana com o intuito de reproduzir as equações relativística com pressão em nível de background e perturbativo.

Considerando-se um fluido simples, as versões modificadas das equações de Euler, da continuidade e de Poisson utilizadas nessa descrição são, respectivamente:

$$
\begin{gathered}
\left(\frac{\partial \mathbf{u}}{\partial t}\right)_{r}+\left(\mathbf{u} \cdot \nabla_{r}\right) \mathbf{u}=-\nabla_{r} \Phi-\frac{\nabla_{r} p_{c}}{\rho+p_{c}} \\
\left(\frac{\partial \rho}{\partial t}\right)_{r}+\nabla_{r} \cdot(\rho \mathbf{u})+p_{c} \nabla_{r} \cdot \mathbf{u}=0
\end{gathered}
$$

e

$$
\nabla_{r}^{2} \Phi=4 \pi G\left(\rho+3 p_{c}\right)
$$

onde $\rho$ é a densidade de matéria escura, $u$ é um campo de velocidade, $\Phi$ é o potencial gravitacional e um termo de pressão de criação, $p_{c}$, fora introduzido.

Foi mostrado que, mesmo no caso $\omega$ dependente do tempo, o conjunto de equações Neo-Newtonianas é capaz de gerar resultados equivalentes aos da abordagem relativística, dentro de certos limites (Reis, 2003a, $\underline{\text { b }}$ ). Logo, essa abordagem cuja a precisão da aproximação foi mostrada para diferentes épocas e até mesmo em escalas da ordem das escalas de Hubble, é capaz de fornecer um razoável insight sobre as perturbações de fluidos com pressão, até mesmo em ordem não linear (Naveri e Padmanabhan, 1998; Abramo et al., 2007; Pace et al., 2010).

\subsubsection{Modelo CCDM na Abordagem Neo-Newtoniana}

Na abordagem Neo-Newtoniana, as equações gravitacionais são tratadas de forma semiclássica. Porém, por incorporar algumas características importantes da abordagem relativística, esse formalismo pode fornecer um razoável entendimento acerca do comportamento de modelos cosmológicos com pressão. Discutimos aqui como o conjunto básico de equações relativísticas do modelo LJO pode ser reobtido através da abordagem NeoNewtoniana (Lima et al., 2011; Jesus et al., 2011). 
Primeiramente, a isotropia e homogeneidade assumida no modelo implica que $\mathbf{u}=H \mathbf{r}$, de acordo com a lei de Hubble. Logo, a equação (6.2) pode ser reescrita como:

$$
\dot{\rho}+3 H \rho=\Gamma \rho,
$$

ou seja, a mesma expressão relativística para a equação de continuidade em modelos com taxa de criação $\Gamma$.

Utilizando a lei de Hubble, a equação de Euler (6.1) pode ser reescrita como

$$
\dot{H} \mathbf{r}+H r \frac{\partial}{\partial r}(H \mathbf{r})=\left(\dot{H}+H^{2}\right) \mathbf{r}=-\nabla_{r} \Phi
$$

enquanto a integração equação de Poisson (6.3) leva a:

$$
\nabla_{r} \Phi=4 \pi G\left(\rho+3 p_{c}\right) \frac{\mathbf{r}}{3},
$$

onde tomamos a relação $p_{c}=-\Gamma \rho /(3 H)$.

Da combinação das equações (6.5) e (6.6), obtemos a equação de aceleração

$$
\frac{\ddot{a}}{a}=-\frac{4 \pi G}{3}\left(\rho+3 p_{c}\right)
$$

onde, em harmonia com o caso relativístico, o termo de pressão é corretamente incorporado nas equações dinâmicas, podendo inclusive, dar origem à expansão acelerada. Da equação (6.4) e usando a definição da pressão de criação, a integração da equação (6.7) resulta em

$$
8 \pi G \rho=3 H^{2}+\frac{3 K}{a^{2}}
$$

onde K é uma constante de integração arbitrária. Tal expressão, nada mais é que a primeira das equações de Friedmann (2.14). Ressaltamos aqui que os resultados obtidos são válidos independentemente da forma da função $\Gamma$, podendo ser generalizados a diversos modelos CCDM. Por outro lado, os resultados obtidos pelo tratamento newtoniano clássico para modelos CCDM apresentam dificuldades mesmo em ordem zero, haja vista que esses não são capazes de contabilizar corretamente o termo de pressão (Lima et al., 2011).

\subsection{Perturbações de Densidade na Formulação Neo-Newtoniana}

Para a análise perturbativa completa em cosmologia, é necessário uma abordagem completamente relativística uma vez que a análise Newtoniana padrão só é válida quando 
as escalas analisadas são de ordens muito menores que a escala de Hubble e os movimentos de velocidade peculiar são muito menores que o fluxo de Hubble (Peebles, 1980).

Uma abordagem capaz de contornar tais limitações é a abordagem Neo-Newtoniana, cujas aplicações não são tão profundas quanto o tratamento baseado na TRG, mas ainda é capaz de fornecer resultados fisicamente interpretáveis acerca do comportamento perturbativo da matéria (Lima et al., 1997: Pace et al., 2010; Fabris e Velten, 2012: Del Popolo et al., 2013).

Começaremos nossa discussão reescrevendo as equações da abordagem Neo-Newtoniana (6.1), (6.2) e (6.3) no sistema comóvel. A coordenada comóvel x se relaciona à coordenada própria $\mathbf{r}$ por:

$$
\mathbf{x}=\frac{\mathbf{r}}{a}
$$

Nesse sistema de coordenadas, as grandezas $\mathbf{u}, \rho$ e $\Phi$ podem ser reescritas como:

$$
\begin{gathered}
\mathbf{u}=\dot{a} \mathbf{x}+a \dot{\mathbf{x}}=\dot{a} \mathbf{x}+\mathbf{v}, \\
\rho=\bar{\rho}(t)[1+\delta(\mathbf{x}, t)], \\
p_{c}=\bar{p}_{c}(t)+\delta p_{c}(\mathbf{x}, t), \\
\Phi=\phi+\frac{2 \pi G a^{2}}{3}\left(\bar{\rho}+3 \bar{p}_{c}\right) x^{2},
\end{gathered}
$$

$\mathbf{v}$ é a velocidade peculiar, $\delta$ é o contraste de densidade da matéria escura, $\delta p_{c}$ é a perturbação do termo de pressão de criação e $\phi$ é a perturbação do potencial gravitacional. As variáveis $\bar{\rho}, \bar{p}_{c}$ são os valores de background da densidade e da pressão de criação, respectivamente.

Seguindo a metodologia padrão, passamos das coordenadas próprias $(\mathbf{r}, t)$ para as coordenadas comóveis $(\mathbf{x}, t)$ e utilizamos as relações:

$$
\nabla_{x} \equiv \nabla=a \nabla_{r}
$$




$$
\left(\frac{\partial}{\partial t}\right)_{x} \equiv \frac{\partial}{\partial t}=\left(\frac{\partial}{\partial t}\right)_{r}+\frac{\dot{a}}{a} \mathbf{x} \cdot \nabla_{x}
$$

As equações (6.1)-(6.3) são então reescritas como:

$$
\begin{gathered}
\frac{\partial \mathbf{v}}{\partial t}+\frac{\dot{a}}{a} \mathbf{v}+\frac{(\mathbf{v} \cdot \nabla) \mathbf{v}}{a}=-\frac{\nabla \phi}{a}-\frac{\nabla \delta p_{c}}{a\left(\rho+p_{c}\right)}, \\
\bar{\rho} \frac{\partial \delta}{\partial t}+3 H\left(\delta p_{c}-\bar{p}_{c} \delta\right)+\frac{\rho+p_{c}}{a} \nabla \cdot \mathbf{v}+\frac{\bar{\rho}}{a} \mathbf{v} \cdot \nabla \delta=0,
\end{gathered}
$$

$$
\nabla^{2} \phi=4 \pi G a^{2}\left(\bar{\rho} \delta+3 \delta p_{c}\right)
$$

As equações (6.16) -(6.18) são obtidas das equações do background não perturbado (4.20), (4.11) e (4.12). Por simplicidade, vamos definir o parâmetro da equação efetiva de estado

$$
\omega \equiv \frac{\bar{p}_{c}}{\bar{\rho}}
$$

e a velocidade efetiva do som

$$
c_{e f f}^{2} \equiv \frac{\delta p_{c}}{\delta \rho}=\frac{\delta p_{c}}{\bar{\rho} \delta}
$$

Assim, as equações (6.16)-(6.18) podem ser reescritas como:

$$
\begin{gathered}
\frac{\partial \mathbf{v}}{\partial t}+H \mathbf{v}+\frac{(\mathbf{v} \cdot \nabla) \mathbf{v}}{a}=-\frac{\nabla \phi}{a}-\frac{\nabla\left(c_{e f f}^{2} \delta\right)}{a\left[1+\omega+\left(1+c_{e f f}^{2}\right) \delta\right]}, \\
\frac{\partial \delta}{\partial t}+3 H\left(c_{e f f}^{2}-\omega\right) \delta+\frac{1+\omega+\left(1+c_{e f f}^{2}\right) \delta}{a} \nabla \cdot \mathbf{v}+\frac{\mathbf{v} \cdot \nabla \delta}{a}=0,
\end{gathered}
$$

$\mathrm{e}$

$$
\nabla^{2} \phi=4 \pi G a^{2} \bar{\rho} \delta\left(1+3 c_{e f f}^{2}\right)
$$

Vale ressaltar que as equações apresentadas acima podem ser reobtidas de outras análises realizadas no mesmo formalismo como, por exemplo, tomando o caso particular de um único fluido com equação de estado (6.19), conforme realizado por Abramo et al. (2007). 
Obteremos agora as perturbações do fluido em primeira ordem. Desprezando os termos de perturbação de segunda ordem, as equações (6.21) e (6.22) tomam a forma

$$
\begin{gathered}
\frac{\partial \mathbf{v}}{\partial t}+H \mathbf{v}=-\frac{\nabla \phi}{a}-\frac{\nabla\left(c_{e f f}^{2} \delta\right)}{a(1+\omega)}, \\
\frac{\partial \delta}{\partial t}+3 H\left(c_{e f f}^{2}-\omega\right) \delta+\frac{1+\omega}{a} \nabla \cdot \mathbf{v}=0 .
\end{gathered}
$$

Combinando-se a equação (6.23) com o divergente da equação (6.24), pode-se verificar que a expressão (6.24) é reduzida à forma:

$$
\frac{\partial \theta}{\partial t}+H \theta=-4 \pi G a \bar{\rho} \delta\left(1+3 c_{e f f}^{2}\right)+\frac{k^{2} c_{e f f}^{2} \delta}{a(1+\omega)},
$$

onde $\theta \equiv \nabla \cdot \mathbf{v}$, assumimos que $c_{e f f}^{2}=c_{e f f}^{2}(t)$ e que a parte espacial do contraste de densidade é tal que $\delta \propto e^{i \mathbf{k} \cdot \mathbf{x}}$. Das equações (6.25) e (6.26), obtemos:

$$
\begin{gathered}
\frac{\partial^{2} \delta}{\partial t^{2}}+\left[H\left(2+3 c_{e f f}^{2}-3 \omega\right)-\frac{\dot{\omega}}{1+\omega}\right] \frac{\partial \delta}{\partial t} \\
+\left\{3\left(\dot{H}+2 H^{2}\right)\left(c_{e f f}^{2}-\omega\right)+3 H\left[c_{e f f}^{2}-\left(1+c_{e f f}^{2}\right) \frac{\dot{\omega}}{1+\omega}\right]\right\} \delta \\
-4 \pi G \bar{\rho}(1+\omega)\left(1+3 c_{e f f}^{2}\right) \delta+\frac{k^{2} c_{e f f}^{2}}{a^{2}} \delta=0
\end{gathered}
$$

cujo resultado é o mesmo encontrado em Reis (2003a), para um fluido com parâmetro de equação de estado $\omega$.

Nos modelos de criação, o parâmetro efetivo de equação de estado é $\omega=-\frac{\Gamma}{3 H}$, deixando a equação (6.27) na forma:

$$
\begin{array}{r}
\frac{\partial^{2} \delta}{\partial t^{2}}+\left[2 H+\Gamma+3 c_{e f f}^{2} H-\frac{\Gamma \dot{H}-H \dot{\Gamma}}{H(3 H-\Gamma)}\right] \frac{\partial \delta}{\partial t} \\
+\left\{3\left(\dot{H}+2 H^{2}\right)\left(c_{e f f}^{2}+\frac{\Gamma}{3 H}\right)+3 H\left[c_{e f f}^{2}-\left(1+c_{e f f}^{2}\right) \frac{\Gamma \dot{H}-H \dot{\Gamma}}{H(3 H-\Gamma)}\right]\right\} \delta \\
-4 \pi G \bar{\rho}\left(1-\frac{\Gamma}{3 H}\right)\left(1+3 c_{e f f}^{2}\right) \delta+\frac{k^{2} c_{e f f}^{2}}{a^{2}} \delta=0
\end{array}
$$

ou: 


$$
\begin{gathered}
\frac{\partial^{2} \delta}{\partial t^{2}}+\left[2 H+\Gamma+3 c_{e f f}^{2} H-\frac{\Gamma \dot{H}-H \dot{\Gamma}}{H(3 H-\Gamma)}\right] \frac{\partial \delta}{\partial t} \\
+\left\{3 H^{2}\left(c_{e f f}^{2}+\frac{\Gamma}{3 H}\right)+3 H\left[c_{e f f}^{2}-\left(1+c_{e f f}^{2}\right) \frac{\Gamma \dot{H}-H \dot{\Gamma}}{H(3 H-\Gamma)}\right]\right\} \delta \\
-4 \pi G \bar{\rho}\left[1-\frac{\Gamma^{2}}{3 H^{2}}+2 c_{e f f}^{2}\left(2-\frac{\Gamma}{H}\right)\right] \delta+\frac{k^{2} c_{e f f}^{2}}{a^{2}} \delta=0
\end{gathered}
$$

onde utilizamos a equação (4.11).

Do ponto de vista numérico, é mais vantajoso escrever a equação (6.29) em termos da variável $\eta \equiv \ln (a(t))$ :

$$
\begin{gathered}
\delta^{\prime \prime}+\left[2+3 c_{e f f}^{2}+\frac{\Gamma+H^{\prime}}{H}-\frac{\Gamma H^{\prime}-H \Gamma^{\prime}}{H(3 H-\Gamma)}\right] \delta^{\prime} \\
+\left\{3\left(c_{e f f}^{2}+c_{e f f}^{2}\right)+\frac{\Gamma}{H}-3\left(1+c_{e f f}^{2}\right) \frac{\Gamma H^{\prime}-H \Gamma^{\prime}}{H(3 H-\Gamma)}\right\} \delta \\
-\frac{4 \pi G \bar{\rho}}{H^{2}}\left[1-\frac{\Gamma^{2}}{3 H^{2}}+2 c_{e f f}^{2}\left(2-\frac{\Gamma}{H}\right)\right] \delta+\frac{k^{2} c_{e f f}^{2} e^{-2 \eta}}{H^{2}} \delta=0,
\end{gathered}
$$

onde cada (') representa uma derivação em relação ao parâmetro $\eta$.

No caso plano $(K=0)$ a equação (6.30) toma a forma

$$
\begin{gathered}
\delta^{\prime \prime}+\left[2+3 c_{e f f}^{2}+\frac{\Gamma+H^{\prime}}{H}-\frac{\Gamma H^{\prime}-H \Gamma^{\prime}}{H(3 H-\Gamma)}\right] \delta^{\prime}+ \\
+\left[\left(\frac{\Gamma}{H}-1\right)\left(\frac{\Gamma}{2 H}+\frac{3}{2}+3 c_{e f f}^{2}\right)+3 c_{e f f}^{2}{ }^{\prime}-3\left(1+c_{e f f}^{2}\right) \frac{\Gamma H^{\prime}-H \Gamma^{\prime}}{H(3 H-\Gamma)}+\frac{k^{2} c_{e f f}^{2} e^{-2 \eta}}{H^{2}}\right] \delta=0,
\end{gathered}
$$

onde usamos que $\frac{4 \pi G \bar{\rho}}{H^{2}}=\frac{3}{2}$.

Nos casos em que as perturbações de pressão podem ser desprezadas, $c_{\text {eff }}^{2} \equiv 0$, temos:

$$
\delta^{\prime \prime}+\left[2+\frac{\Gamma+H^{\prime}}{H}-\frac{\Gamma H^{\prime}-H \Gamma^{\prime}}{H(3 H-\Gamma)}\right] \delta^{\prime}+\left[\left(\frac{\Gamma}{H}-1\right)\left(\frac{\Gamma}{2 H}+\frac{3}{2}\right)-3 \frac{\Gamma H^{\prime}-H \Gamma^{\prime}}{H(3 H-\Gamma)}\right] \delta=0 .
$$

A expressão (6.31) foi derivada de forma generalizada, podendo ser utilizada em qualquer modelo CCDM plano. 


\subsection{Perturbações de Densidade no Modelo LJO}

Focaremos nossa atenção agora nas perturbações de densidade no modelo de Lima, Jesus \& Oliveira (LJO), caracterizado pela taxa de criação (Lima et al., 2010):

$$
\Gamma=3 \alpha\left(\frac{\rho_{c 0}}{\rho}\right) H
$$

e com o parâmetro de Hubble dado por

$$
H=H_{0}\left[\left(\Omega_{m}-\alpha\right) a^{-3}+\alpha+\left(1-\Omega_{m}\right) a^{-2}\right]^{1 / 2},
$$

sendo $\Omega_{m} \equiv \frac{\rho_{0}}{\rho_{c 0}}$. Lembrando que no caso plano $\Omega_{m}=1$, temos:

$$
H=H_{0}\left[(1-\alpha) a^{-3}+\alpha\right]^{1 / 2}
$$

Assumindo a taxa de criação em (6.33), a equação de perturbação linear de densidade assume a forma (6.30):

$$
\delta^{\prime \prime}+F(\eta) \delta^{\prime}+G(\eta) \delta=0
$$

sendo as funções $F(\eta)$ e $G(\eta)$ dadas no caso plano por (6.35):

$$
\begin{aligned}
F(\eta) & =\frac{(1-\alpha)\left(1+6 c_{e f f}^{2}\right)+2 \alpha e^{3 \eta}\left(8+3 c_{e f f}^{2}\right)}{2\left(1-\alpha+\alpha e^{3 \eta}\right)} \\
G(\eta) & =\frac{3 \alpha e^{3 \eta}\left(5+5 c_{e f f}^{2}+c_{e f f}^{2}\right)-3(1-\alpha)\left(2+c_{e f f}^{2}-c_{e f f}^{2}\right)}{1-\alpha+\alpha e^{3 \eta}} \\
& +\frac{9(1-\alpha)^{2}}{2\left(1-\alpha+\alpha e^{3 \eta}\right)^{2}}+\frac{e^{\eta} k^{2} c_{e f f}^{2}}{H_{0}^{2}\left(1-\alpha+\alpha e^{3 \eta}\right)}
\end{aligned}
$$

Conforme as deduções realizadas, o cálculo do contraste de densidade passa pela resolução numérica da equação diferencial de segunda ordem (6.36). Como condição inicial, em altos redshifts o Universo deve se comportar como o previsto pelo modelo de Einstein de Sitter. Dessa forma, adotamos $\delta\left(a_{i}\right)=a_{i}$ e $\delta^{\prime}\left(a_{i}\right)=1$, onde $a_{i}=10^{-3}$ (Peebles, 1980). Tal condição é naturalmente satisfeita pelo modelo de LJO, haja vista que o fenômeno de criação é desprezível em altos redshifts e o modelo tende ao cenário cosmológico composto apenas por matéria sem pressão.

Além disso, a integração da função $\delta$ requer uma escolha de $c_{e f f}^{2}$ ou, equivalentemente, da perturbação da pressão de criação. Iniciaremos considerando $\delta p_{c}$ como um novo grau de 
liberdade no modelo. independente das variáveis do fluido (Abramo et al.. 2007: Garriga e Mukhanov, 1999; Ellis et al., 2012). Dada a forma da pressão de criação, podemos discutir algumas formas funcionais para $\delta p_{c}$. No modelo LJO, a pressão de criação é:

$$
p_{c}=-\alpha \rho_{c 0}
$$

sendo uma constante temporal. Logo, uma escolha natural é $\delta p_{c}=0 \mathrm{ou}$, em outras palavras, $c_{\text {eff }}^{2}=0$. Considerando-se essa escolha nas expressões (6.37) e (6.39), obtemos:

$$
\begin{aligned}
& F(\eta)=\frac{(1-\alpha)+16 \alpha e^{3 \eta}}{2\left(1-\alpha+\alpha e^{3 \eta}\right)}, \\
& G(\eta)=\frac{9(1-\alpha)^{2}}{2\left(1-\alpha+\alpha e^{3 \eta}\right)^{2}}+\frac{3\left(5 \alpha e^{3 \eta}-2+2 \alpha\right)}{1-\alpha+\alpha e^{3 \eta}} .
\end{aligned}
$$

Esse caso é também interessante uma vez que as equações perturbadas Neo-Newtonianas obtidas aqui são equivalentes às derivadas via TRG para um fluido único $\operatorname{com} c_{e f f}^{2}=0$ (Reis, 2003a, b)

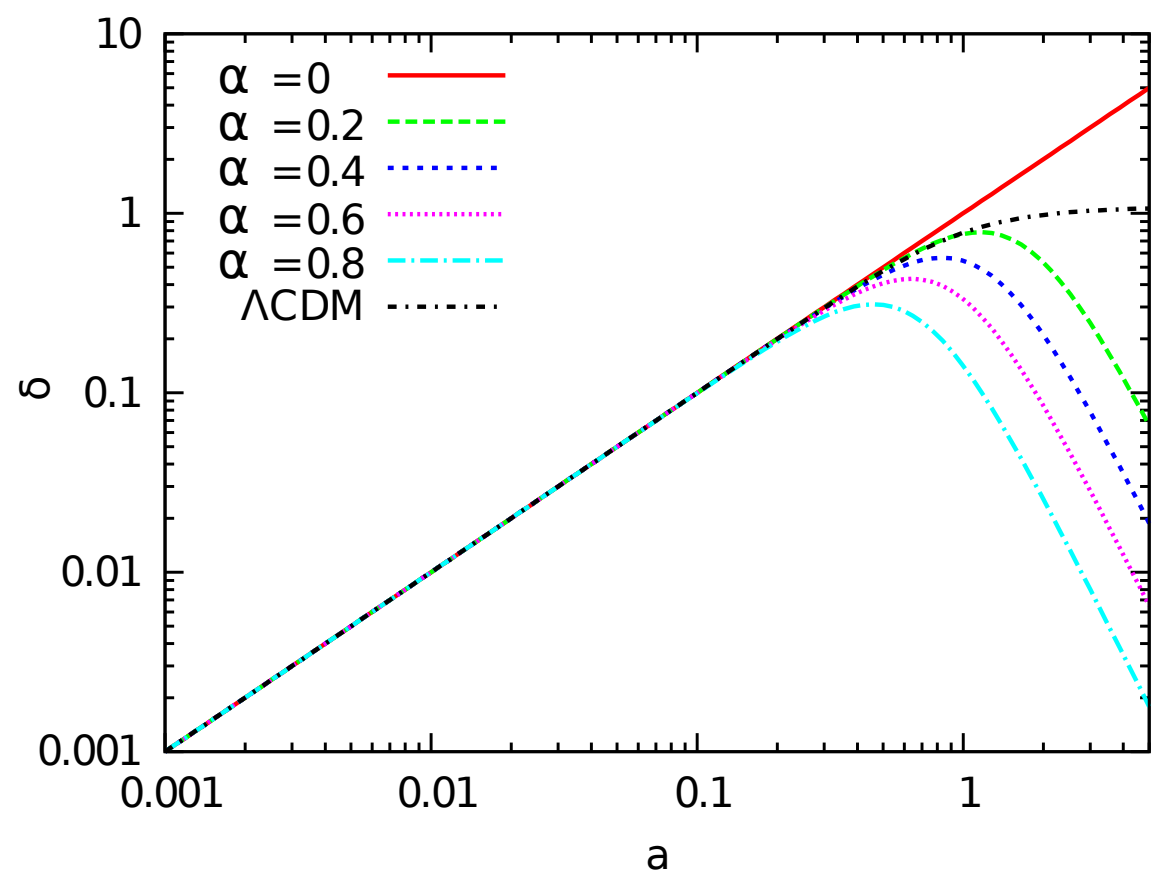

Figura 6.1: Contraste de densidade $\delta$ em função de $a$ para o caso $c_{e f f}^{2}=0$. Para o modelo LJO, este caso é equivalente a $c_{e f f}^{2}=c_{s}^{2}$ pois a velocidade do som adiabática é zero. Comparamos também o caso $\Lambda \mathrm{CDM}$ plano, com $\Omega_{m}=1-\Omega_{\Lambda}=0.3$ e $c_{e f f}^{2}=0$.

Outra possibilidade interessante é a escolha da velocidade efetiva do som como $c_{e f f}^{2}=$ $c_{s}^{2}=\dot{p}_{c} / \dot{\rho}$. Neste caso, a parte não adiabática da pressão $\delta p_{n a d} \approx \delta p_{c}-c_{s}^{2} \delta \rho$ se anula. Se 
interpretarmos o modelo efetivamente como um fluido de densidade $\rho$ e pressão $p_{c}$ obtemos um regime de perturbações puramente adiabáticas. A velocidade do som obtida portanto é:

$$
c_{s}^{2}=\frac{H \Gamma^{\prime}-\Gamma H^{\prime}-\Gamma(3 H-\Gamma)}{3 H(3 H-\Gamma)},
$$

Uma vez que a pressão de criação no modelo LJO é constante $\left(p_{c}=-\alpha \rho_{c 0}\right)$, então $c_{s}^{2}=0$ e esta escolha acaba por se reduzir ao caso anteriormente discutido.

Outra possibilidade interessante é associar o velocidade efetiva do som a uma variável dependente do tempo como, por exemplo, $c_{\text {eff }}^{2}=\omega$. Novamente, devemos ressaltar que $c_{\text {eff }}^{2}$ está sendo tratado como um novo grau de liberdade. Esse tratamento é relevante quando as equações do fluido evoluem fora do equilíbrio termodinâmico, como no caso do modelo abordado.

Na figura 6.1, apresentamos a evolução do contraste de densidade para o modelo LJO, dentro do formalismo Neo-Newtoniano. Neste caso, o modo de crescimento da perturbação atinge um valor máximo e então passa a ser suprimido. Particularmente, para $\alpha \approx 0.2$, o contraste de densidade previsto pelo modelo é praticamente indistinguível da previsão dada pela cosmologia $\Lambda$ CDM. Entretanto, este valor é completamente conflitante com as previsões em background ( $\alpha \approx 0.7)$ Lima et al. (2010).

Na figura 6.2, mostramos as soluções para $\delta$ obtidas no caso $c_{\text {eff }}^{2}=\omega=-\Gamma / 3 H$. Conforme mostrado, o contraste de densidade obtido para diferentes valores de $\alpha$ só possui comportamento similar ao encontrado no modelo $\Lambda$ CDM, para $0.2 \leq \alpha \leq 0.4$.

Naturalmente, uma outra possibilidade interessante é considerar que $c_{\text {eff }}^{2}$ seja um parâmetro livre constante do modelo e, portanto, permitir que a análise observacional encontre os seus valores de melhor ajuste. Seguindo essa consideração, obtemos:

$$
\begin{aligned}
& F(\eta)=\frac{(1-\alpha)\left(1+6 c_{e f f}^{2}\right)+2 \alpha e^{3 \eta}\left(8+3 c_{e f f}^{2}\right)}{2\left(1-\alpha+\alpha e^{3 \eta}\right)} \\
& G(\eta)=\frac{9(1-\alpha)^{2}}{2\left(1-\alpha+\alpha e^{3 \eta}\right)^{2}}+\frac{15 \alpha e^{3 \eta}\left(1+c_{e f f}^{2}\right)-3(1-\alpha)\left(2+c_{e f f}^{2}\right)}{1-\alpha+\alpha e^{3 \eta}}+\frac{e^{\eta} k^{2} c_{e f f}^{2}(6-45)}{H_{0}^{2}\left(1-\alpha+\alpha e^{3 \eta}\right)}
\end{aligned}
$$

Na figura 6.3, mostramos a evolução do contraste de densidade quando $c_{\text {eff }}^{2}$ é tomado como um parâmetro livre do modelo. Os valores selecionados podem ser verificados na mesma figura. Novamente, vemos que, dentro de um certo limite de valores, a flutuação de matéria reproduz o comportamento predito pelo modelo $\Lambda$ CDM. 


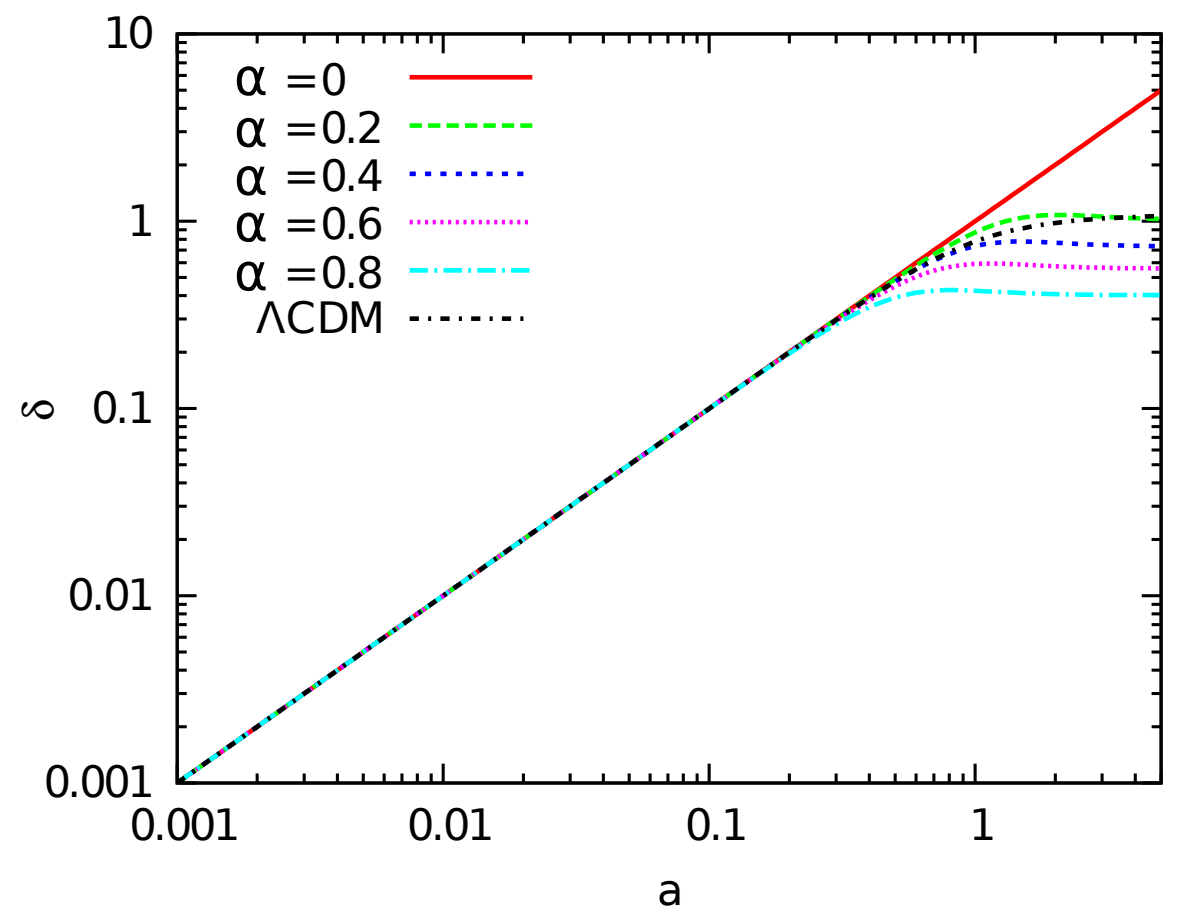

Figura 6.2: Contraste de densidade $\delta$ em função de $a$ para o caso $c_{e f f}^{2}=\omega$. Por comparação, mostramos a solução para o caso $\Lambda$ CDM plano, com a identificação $\Omega_{m}=1-\alpha=0.3$ e $c_{e f f}^{2}=0$. As curvas obtidas foram tomadas no limite de grandes escalas $(k \rightarrow 0)$.

\subsection{Análise Observacional e a Taxa de Crescimento das Perturbações}

Nesta seção discutimos a aglomeração da matéria escura utilizando a evolução da taxa de crescimento linear como indicador (Peebles, 1993). Essa parametrização das flutuações de densidades $\delta(z)$ tem a seguinte forma:

$$
f(z)=\frac{d \ln \delta}{d \ln a}=-(1+z) \frac{d \ln \delta}{d z} .
$$

Conforme pode ser notado pela expressão (6.46), dados dessa natureza fornecem vínculos independentes de $H_{0}$. Na Tabela 6.1, são mostrados os dados da taxa de crescimento que utilizaremos mais a frente em nossa análise, suas incertezas, redshifts e referências bibliográficas.

Obtemos os limites sobre os parâmetros relevantes do modelo utilizando o procedimento de minimização de $\chi^{2}$, por meio da comparação de dados observacionais da taxa de crescimento com as previsões do modelo LJO discutidas nas seções anteriores. O valor de melhor ajuste do conjunto de parâmetros livres $\left(\alpha, c_{e f f}^{2}\right)$ pode ser estimado via um teste estatístico de $\chi^{2}$ : 


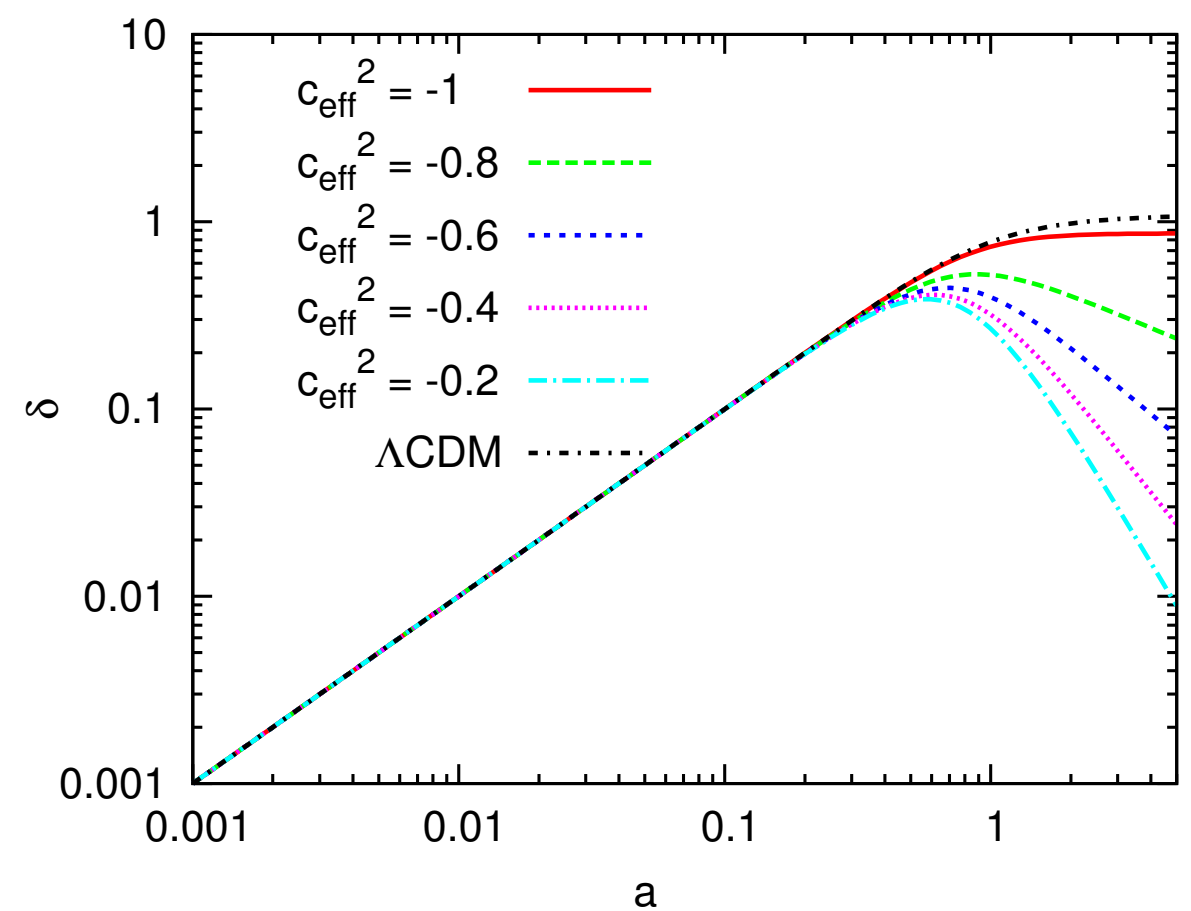

Figura 6.3: Contraste de densidade $\delta$ em função de $a$ para o caso em que $c_{\text {eff }}^{2}$ é um parâmetro livre. Adicionalmente, mostramos o caso $\Lambda \mathrm{CDM}$ plano, com $\Omega_{m}=0.3$ e $c_{e f f}^{2}=0$. As curvas mostradas foram obtidas para o limite de grandes escalas $(k \rightarrow 0)$ e $\alpha=0.7$.

$$
\chi^{2}\left(\alpha, c_{e f f}^{2}\right)=\sum_{i=1}^{7}\left[\frac{f_{o b s}\left(z_{i}\right)-f_{\mathrm{LJO}}\left(z_{i}, \alpha, c_{e f f}^{2}\right)}{\sigma_{i}}\right]^{2},
$$

onde $\sigma_{i}$ é incerteza da taxa de crescimento. Utilizamos como priors $\alpha \in[0.1,1]$ e $c_{\text {eff }}^{2} \in$ $[-1.3,2]$. Os resultados da análise estatística são mostrados na figura 6.4, onde as regiões no plano $\alpha-c_{\text {eff }}^{2}$ delimitam os valores permitidos em $68.3 \%, 95.4 \%$ e $99.7 \%$ de nível de confiança estatística. Notemos que o valor de $c_{e f f}^{2}$ não é bem restrito pela análise estatística, tendo todos os seus valores no intervalo $[-1.3,2]$ permitidos dentro de $1 \sigma$ de incerteza.

A análise de verossimilhança é capaz de impor um vínculo mais restritivo sobre $c_{e f f}^{2}$ quando adicionamos a informação de que o universo deva ter um redshift de transição delimitando uma época no passado em que houve a mudança entre o estado desacelerado e o presente estado acelerado de expansão cósmica. No caso $\alpha>\frac{1}{3}$, a velocidade efetiva do som permanece no intervalo $-1.24 \leq c_{e f f}^{2} \leq-0.07$ com $95.4 \%$ de confiança estatística.

Ainda nesse mesmo caso, ao marginalizarmos sobre $\alpha$, a análise de verossimilhança fornece como valor de melhor ajuste $c_{e f f}^{2} \simeq-1 \operatorname{com} \chi^{2}=4.03$ e $\chi_{\nu}^{2}=0.67$, com 6 graus de 

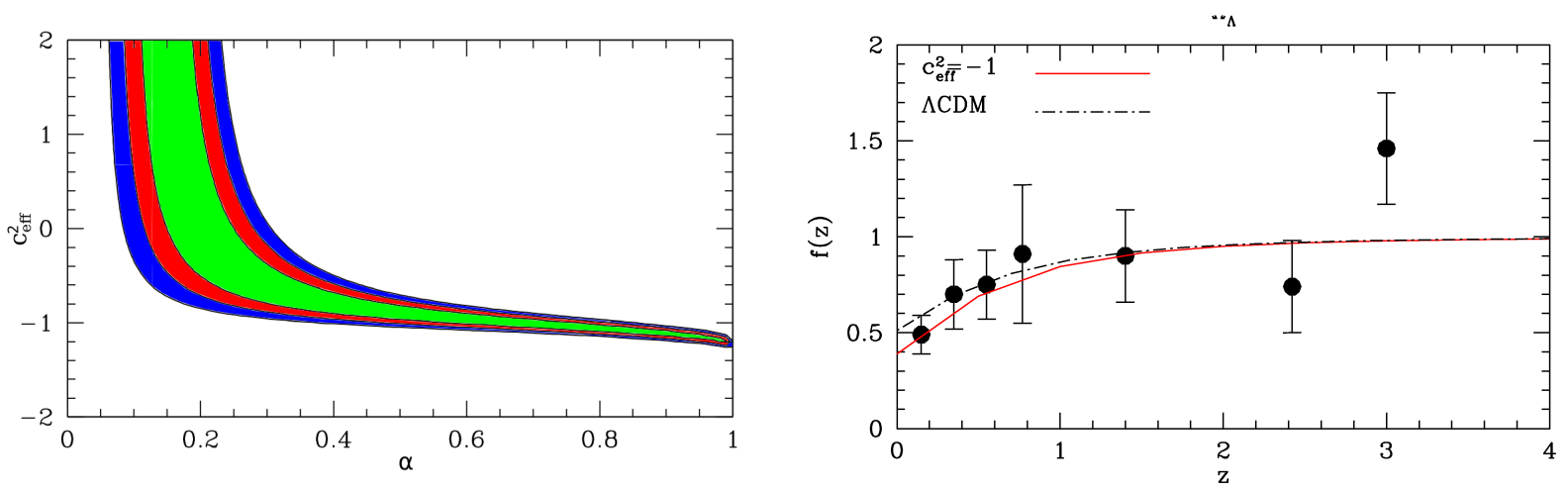

Figura 6.4: Esquerda:) Plano $c_{e f f}^{2}-\alpha$ para o modelo LJO plano considerando-se dados de $f(z)$. As regiões delimitam $68.3 \%, 95.4 \%$ e $99.7 \%$ de confiança estatística. Direita:) Medidas de $f(z)$ e curvas teóricas obtidas com os valores de melhor ajuste do modelo LJO (linha sólida vermelha) e do modelo $\Lambda$ CDM (linha tracejada preta) (Jesus et al., 2011).

liberdade. A mérito de comparação, a mesma análise foi realizada para o modelo $\Lambda$ CDM no qual obtivemos $\chi^{2}=4.88$ e $\chi_{\nu}^{2}=0.70$.

Adicionalmente, comparamos os resultados estatísticos obtidos nos modelos LJO e $\Lambda \mathrm{CDM}$ utilizando o critério de informação Bayesiana (Bayesian Information Criterion $\mathrm{BIC}$ ), definido como $B I C=\chi^{2}+k_{n} \ln N$, onde $k_{n}$ é o número de parâmetros livres do modelo e $N \equiv 7$ é o número de dados observacionais utilizados na análise (Schwarz, 1978). Como critério de seleção entre modelos, utilizamos o estimador $\triangle B I C=B I C_{L J O}-B I C_{\Lambda C D M}$. Valores $\triangle B I C>2$ indicam evidência contra o modelo testado e valores $\Delta B I C>6$ corresponde a evidências fortes contra o modelo. No nosso caso, encontramos $\triangle B I C \simeq 1.1$ indicando que o modelo LJO com $\left(\Omega_{k}, \alpha, c_{e f f}^{2}\right)=(0.00,0.70,-1)$ ajusta muito bem os dados de fator de crescimento, ainda que penalizado por ter um parâmetro livre a mais que o modelo $\Lambda$ CDM.

\begin{tabular}{|c|c|c|}
\hline $\mathrm{z}$ & $f_{\text {obs }}$ & Referência \\
\hline 0.15 & $0.49 \pm 0.10$ & Colless et al. (2001); Guzzo et al. (2008) \\
0.35 & $0.70 \pm 0.18$ & Tegmark et al. (2006) \\
0.55 & $0.75 \pm 0.18$ & Ross et al. (2007) \\
0.77 & $0.91 \pm 0.36$ & Guzzo et al. (2008) \\
1.40 & $0.90 \pm 0.24$ & da Angela et al. (2008) \\
2.42 & $0.74 \pm 0.24$ & Viel et al. (2004) \\
3.00 & $1.46 \pm 0.29$ & McDonald et al. (2005) \\
\hline
\end{tabular}

Tabela 6.1 - Dados da taxa de crescimento $f_{\text {obs }}$ em função do redshift e suas respectivas referências. 
Os testes estatísticos discutidos indicam que o modelo LJO plano com os parâmetros $0.65 \leq \alpha \leq 0.74$ e $c_{e f f}^{2}=-1$ reproduzem a expansão observada do Universo. Dentro da análise Neo-Newtoniana, mostramos que o modelo é capaz de descrever o campo de flutuação de densidades da matéria modo semelhante ao modelo $\Lambda$ CDM e sem a necessidade de uma componente de energia escura.

\subsection{Discussões}

Os problemas relacionados à natureza das componentes do setor escuro do universo passam necessariamente pela determinação da natureza da matéria escura não-bariônica. Dentro da TRG, é uma questão relevante discutir se matéria e energia escura podem ser unificadas em uma única componente escura, com o modelo $\Lambda$ CDM emergindo como uma descrição efetiva (Lima et al., 2010). Claramente, um modelo de matéria não relativística, como o caso do Modelo de Einstein-de Sitter, não é capaz de descrever as observações a que temos acesso atualmente. Em particular, um modelo sem um fluido exótico em um background homogêneo e isotrópico deve apresentar algum mecanismo que permita o estágio acelerado de expansão.

Dentro do cenário apresentado, desenvolvemos o sistema de equações para as perturbações lineares de densidade em modelos CCDM. Nesses modelos cosmológicos, a pressão negativa é relacionada à taxa de criação através da expressão $p_{c}=-\Gamma \rho /(3 H)$. Assumimos em nossa descrição uma equivalência completa entre o fluido de matéria escura no modelo e um fluido cuja equação de estado efetiva $\omega=-\Gamma / 3 H$ que pode, em geral, ser dependente do tempo. As equações resultantes dessa abordagem permanecem válidas independentemente da escolha de $\Gamma$.

Aplicamos a descrição obtida na investigação das perturbações lineares no caso plano do modelo com criação de matéria escura de Lima et al. (2010), cuja expansão cósmica prevista é equivalente à do modelo $\Lambda$ CDM. Três casos perturbativos particulares, classificados em função da forma da velocidade efetiva do som $c_{e f f}^{2}$, foram discutidos.

No primeiro caso, assumindo $c_{e f f}^{2}=c_{s}^{2}=0$, mostramos que os modelos LJO e $\Lambda$ CDM produzem resultados similares apenas antes da manifestação da energia escura/pressão de criação, i.e., até redshifts da ordem de $z \sim 1$. Desse redshift em diante, os modos de crescimento de perturbação decaem fortemente, com atenuação crescente com o aumento 
do valor do parâmetro de criação $\alpha$, conforme a figura (6.1). O caso adiabático, $c_{\text {eff }}^{2}=\omega$, apresentou equivalência entre os modelos LJO e $\Lambda$ CDM perturbativos porém, apenas no limite $0.2 \leq \alpha \leq 0.4$, conforme a figura (6.2). Por último, assumindo $c_{\text {eff }}^{2} \equiv \delta p / \delta \rho$ como um parâmetro livre constante (Abramo et al., 2007), a evolução do modelo LJO perturbativo é similar àquela prevista pelo modelo $\Lambda \mathrm{CDM}$, para $\alpha=0.7$, em concordância com os vínculos em background (figura 6.3).

Limitamos os parâmetros livres $\alpha$ e $c_{e f f}^{2}$ do modelo LJO plano através de um teste estatístico de $\chi^{2}$, utilizando dados proveniente da taxa de crescimento das aglomerações. Os valores de melhor ajuste obtidos foram $\alpha=0.7$ e $c_{\text {eff }}^{2}=-1$, em harmonia com os vínculos obtidos em background (Lima et al., 2010). Dentro do formalismo desenvolvido, pode-se afirmar que os modelos $\Lambda$ CDM e LJO predizem, além da mesma história de expansão cósmica, dinâmicas de flutuação de matéria escura similares.

Por outro lado, embora os dados observacionais privilegiem um modelo de criação com $c_{\text {eff }}^{2} \approx-1$, esse valor de velocidade efetiva deve gerar instabilidades nas perturbações de densidades. Entretanto, no modelo com $c_{e f f}^{2} \approx 0$, as perturbações de densidades sofrem forte supressão, a partir de $z \approx 1$.

Um possível meio de contornar essas dificuldades é assumir que a matéria escura fria possa se decompor em uma componente aglomerativa e outra componente homogênea. Essa possibilidade fora originalmente sugerida em Lima et al. (2010), inspirados pelas medidas de raios-X em aglomerados, e plenamente desenvolvida por Ramos et al. (2014) e Vargas dos Santos et al. (2014). Particularmente, Ramos et al. (2014) considerou a formulação relativística para a obtenção das perturbações lineares da matéria escura no caso adiabático $\left(\operatorname{com} c_{e f f}^{2}=c_{s}^{2}=0\right)$. Na análise realizada mostrou-se que, ao assumir toda a matéria escura colapsando, o contraste de densidade da matéria $\delta_{c c d m}$ no modelo com criação é suprimido por um fator

$$
\delta_{c c d m} / \delta_{m, \Lambda c d m}=\frac{(1-\alpha) a^{-3}}{\alpha+(1-\alpha) a^{-3}},
$$

em relação ao contraste de densidade da matéria escura do modelo $\Lambda$ CDM. A evolução da taxa de crescimento $\delta_{c c d m}$ passa então a ser suprimida a partir do redshift $z \sim 1$, sendo a intensidade dessa supressão fortemente dependente do valor da constante $\alpha$. Contudo, é possível decompor a componente de matéria escura $\rho_{m}=\left(\rho_{m 0}-\alpha \rho_{c 0}\right) a^{-3}+\alpha \rho_{c 0}$ no modelo CCDM em duas componentes, a saber (Ramos et al., 2014), (i) uma componente 
de matéria escura sem pressão e inomogênea, tal que:

$$
\dot{\rho}_{\text {inom }}+3 H \rho_{\text {inom }}=0
$$

e (ii) uma segunda componente de matéria escura com pressão negativa e comportamento "suave", tal que:

$$
\dot{\rho}_{\text {hom }}+3 H\left(\rho_{\text {hom }}+p_{\text {hom }}\right)=0 .
$$

A mérito de comparação com as observações, as medidas relacionadas à formação de estruturas passam a ser dependentes do termo $\rho_{c l}$ e o contraste de densidade passa a ser dado por

$$
\delta_{c l}=\delta \rho / \rho_{c l}
$$

Conforme discutido por Ramos et al. (2014), o contraste de densidade $\delta_{c l}$ fornece ótimo ajuste aos dados disponíveis de $f(z) \sigma_{8}(z)$, uma vez que, nessa interpretação, os modelos CCDM e $\Lambda$ CDM passam a ser degenerados, mesmo em nível perturbativo. Essa caraterística é referida pelos autores como deaenerescência escura (Ramos et al.. 2014: Vargas dos Santos et al., 2014).

Devemos ressaltar que, ainda que se possa considerar os modelos LJO e $\Lambda$ CDM cosmologicamente degenerados, no campo da Teoria Quântica de Campos em Espaços Curvos (TQCEC), esses modelos se baseiam em fenômenos completamente distintos. Em princípio, através da TQCEC é possível limitar observacionalmente o valor da massa da partícula de matéria escura, assim como a taxa de criação de partículas dada uma evolução do fator de escala cósmico, conforme a proposta de Jesus e Pereira (2014).O grande desafio teórico dessa metodologia é contabilizar os efeitos de backreaction inerentes ao cenário.

Em síntese, o cenário cosmológico com criação de LJO é capaz de reproduzir a história cósmica e ser comparável com observações cosmológicas, porém ainda sendo um cenário fortemente fenomenológico. Esperamos que no futuro, através das previsões obtidas nos diferentes formalismos e do desenvolvimento das abordagens teóricas e de medidas observacionais, possamos discutir um cenário completo, refutável e não degenerado com o modelo padrão. 
Capítulo 7

\section{Um Novo Modelo CCDM com Curvatura Espacial}

\subsection{Introdução}

Prosseguiremos com as nossas discussões acerca da natureza da energia escura e da viabilidade de modelos com criação de matéria. Uma vez que o modelo $\Lambda$ CDM é capaz de descrever a maior parte das observações, naturalmente espera-se que modelos cosmológicos bem sucedidos devam produzir previsões cosmológicas razoavelmente semelhante.

Mostramos nos capítulos anteriores que a taxa de criação fenomenológica proposta no modelo LJO dá origem a um modelo apto a explicar grande parte das observações cosmológicas. Em contrapartida, esse modelo é degenerado com o modelo $\Lambda$ CDM em background e perturbativamente, conforme vem sendo discutido na literatura (Lima et al., 2010; Jesus et al., 2011; Ramos et al., 2014). Essa questão vem sendo chamada de "Degenerescência Escura" (Dark Degeneracy) (Ramos et al., 2014; Vargas dos Santos et al., 2014).

Neste capítulo, discutimos uma nova possibilidade dentro do cenário CCDM, onde exploramos um modelo cuja taxa de criação é proporcional a $H^{-1}$. O modelo apresentado aqui possui mesmo grau de liberdade e semelhanças dinâmicas com os modelos $\Lambda$ CDM e LJO. Porém, nessa proposta, a existência de um termo de curvatura no universo gera previsões significantemente diferentes das previsões obtidas no modelo LJO. Além disso, mostramos que esse modelo prevê uma dinâmica acelerada, compatível com as observações em background e não degeneradas com os casos discutidos anteriormente (Jesus e AndradeOliveira, F., 2015). Na seção 7.2, caracterizamos o modelo de criação e discutimos a sua dinâmica básica. Os vínculos obtidos sobre os parâmetros livres são apresentados na seção 7.3. Os resultados finais e as discussões são mostrados na seção 7.4 . 


\subsection{Dinâmica Cósmica}

Uma dependência natural para a taxa de criação em modelos CCDM é com a taxa de expansão cósmica, conforme já proposto na literatura, em regime de dependência linear (Degeneracy between CCDM and $\$ \backslash$ Lambda $\$ C D M$ cosmologies, lim) e seguindo uma lei de potências do parâmetro de Hubble (Lima et al., 2014).

Claramente, um modelo CCDM viável deve ter em consideração que a aceleração da expansão é um fenômeno recente e, consequentemente, o mecanismo de criação deve se manifestar em redshifts menores. Haja vista que, conforme o Universo evolui, o parâmetro de Hubble decresce, é interessante investigar uma taxa de criação $\Gamma$ cuja dependência com esse parâmetro seja uma lei de potências negativas. Como caso mais simples, propomos aqui um modelo cosmológico com a seguinte taxa de criacão (Jesus e Andrade-Oliveira, F., 2015):

$$
\Gamma=3 \alpha \frac{H_{0}^{2}}{H}
$$

onde $\alpha$ é um parâmetro livre constante adimensional e o fator $3 H_{0}$ foi inserido por conveniência matemática.

A pressão de criação fornecida pelo presente modelo é (equação 4.20):

$$
p_{c}=-\frac{\alpha \rho H_{0}^{2}}{H^{2}} .
$$

Inserindo a equação (7.2) em (4.11) e (4.12), as equações de Friedmann para o modelo ficam:

$$
8 \pi G\left(\rho_{d m}+\rho_{b}+\rho_{r}\right)=3 \frac{\dot{a}^{2}}{a^{2}}+3 \frac{K}{a^{2}}
$$

e

$$
8 \pi G\left(\rho_{d m} \frac{\Gamma}{3 H}+p_{r}\right)=2 \frac{\ddot{a}}{a}+\frac{\dot{a}^{2}}{a^{2}}+\frac{K}{a^{2}},
$$

onde $\rho_{d m}, \rho_{b} \mathrm{e} \rho_{r}$ são respectivamente as densidades de matéria escura fria, bárions e radiação, $p_{r}=\rho_{r} / 3$ é a pressão de radiação e utilizamos a equação (4.20). Visto que estamos considerando exclusivamente as fases mais recentes do Universo, podemos desprezar os termos de radiação deste ponto em diante.

Combinando-se as equações (7.3) e (7.4), obtemos: 


$$
2 \ddot{a} a=-\left(H^{2}+\frac{K}{a^{2}}\right)\left(1-\frac{\Gamma}{H}\right)-\frac{8 \pi G \rho_{b}}{3} \frac{\Gamma}{H} .
$$

A taxa de criação $\Gamma$ não afeta a conservação bariônica usual e, portanto, $\rho_{b}=\rho_{b 0}(1+z)^{3}$. Logo, manipulando a equação (7.5) e utilizando as definições do parâmetro de densidade de bárions atual $\Omega_{b}=\rho_{b 0} / \rho_{c 0}$ e do parâmetro de densidade de curvatura atual $\Omega_{k}=-K / H_{0}^{2}$, obtemos:

$$
\dot{H}=-H^{2}-H_{0}^{2} \Omega_{b}(1+z)^{3} \frac{\Gamma}{H}-\left[H^{2}-\Omega_{k}(1+z)^{2}\right]\left(1-\frac{\Gamma}{H}\right)
$$

onde usamos que $\ddot{a} / a=\dot{H}+H^{2}$. A evolução de $H$, em termos do redshift é

$$
\frac{d H}{d z}=\frac{H}{1+z}+\frac{H_{0}^{2} \Omega_{b}(1+z)^{2} \Gamma}{2 H^{2}}+\frac{H^{2}-H_{0}^{2} \Omega_{k}(1+z)^{2}}{2 H(1+z)}\left(1-\frac{\Gamma}{H}\right) .
$$

Utilizando as definições da taxa de criação em (7.2) e definindo a variável adimensional $y \equiv\left(H / H_{0}\right)^{2}$, a equação (17.7) toma a forma:

$$
\frac{d y}{d z}=3 \frac{y-\alpha}{1+z}+\frac{3 \alpha \Omega_{b}(1+z)^{2}}{y}-\Omega_{k}(1+z)\left(1-\frac{3 \alpha}{y}\right),
$$

com a condição inicial $y(z=0)=1$. A equação (7.8) não possui solução analítica, exceto em alguns de seus casos particulares. Por exemplo, para um universo plano e desprezandose a contribuição dos bárions, i. e., $\Omega_{b}=\Omega_{k}=0$, a solução de (17.8) é

$$
H^{2}(z)=H_{0}^{2}\left[(1-\alpha)(1+z)^{3}+\alpha\right]
$$

ou seja, a solução tem a mesma forma funcional que os parâmetros de Hubble obtidos nos modelos LJO plano ou no modelo $\Lambda$ CDM plano (a menos da correspondência $\Omega_{\Lambda}=\alpha$ ). Uma vez que a equivalência dinâmica só ocorre quando a contribuição dos bárions é negligenciada, naturalmente no modelo proposto aqui é possível discriminar-se entre o cenário com criação e o cenário com constante cosmológica em que se encontra o modelo $\Lambda \mathrm{CDM}$, mesmo em nível de background. Essa condição fica ainda mais clara ao expressarmos a equação diferencial para o parâmetro de Hubble no modelo $\Lambda$ CDM:

$$
\frac{d H}{d z}=\frac{3}{2} \frac{H}{1+z}-\frac{3}{2} \frac{H_{0}^{2} \Omega_{\Lambda}}{H(1+z)}-\frac{H_{0}^{2} \Omega_{k}(1+z)}{2 H}
$$

ou ainda, em termos de $y=H / H_{0}$ : 


$$
\frac{d y}{d z}=3 \frac{y-\Omega_{\Lambda}}{1+z}-\Omega_{k}(1+z)
$$

Da comparação entre as equações (7.8) e (7.11), concluímos que o modelo aqui proposto possui como casos particulares (i) o modelo $\Lambda$ CDM plano, com $\Omega_{k}=\Omega_{b}=0$, tomando a identificação $\alpha=\Omega_{\Lambda}$ e (ii) o modelo de Einstein-de Sitter, com $\Omega_{k}=\alpha=0$.

Outra solução analítica pode ser obtida para o caso plano $\left(\Omega_{k}=0\right)$, mas com a inclusão da densidade de bárions $\left(\Omega_{b} \neq 0\right)$. Nesse caso, a equação (7.8) tem como solução:

$$
\left(\frac{H}{H_{0}}\right)^{2}=\Omega_{b}(1+z)^{3}\left[1+W\left(\frac{\left(1-\Omega_{b}\right) e^{\frac{1-\alpha}{\Omega_{b}}+\frac{\alpha}{(1+z)^{3} \Omega_{b}}-1}}{\Omega_{b}}\right)\right]
$$

onde $W(x)$ é a função principal de Lambert, definida como a solução da equação $x=$ $W(x) e^{W(x)}$.

Em contrapartida, quando consideramos um universo com curvatura e desconsideramos a contribuição dos bárions $\left(\Omega_{b}=0, \Omega_{k} \neq 0\right)$, o parâmetro de Hubble do modelo não possui expressão analítica. Porém, se as contribuições da matéria bariônica e do parâmetro de curvatura forem pequenas, i.e., $0<\Omega_{b} \ll 1,\left|\Omega_{k}\right| \ll 1$, podemos utilizar a seguinte aproximação:

$$
\begin{aligned}
\left(\frac{H}{H_{0}}\right)^{2} & =\alpha+(1-\alpha)(1+z)^{3}+\frac{\Omega_{k}}{2}\left[1-(1+z)^{2}\right]+3 \Omega_{k} \log (1+z)+ \\
& +\left(\frac{\Omega_{b} \alpha}{1-\alpha}-\Omega_{k}\right) \log \left[\alpha+(1-\alpha)(1+z)^{3}\right]
\end{aligned}
$$

Na figura 7.1, são mostradas algumas das soluções numéricas para a razão $H(z) / H_{0}$ variando-se os valores dos parâmetros livres $\alpha$ e $\Omega_{k}$. Nos casos apresentados, fixamos o valor de $\Omega_{b}=0.049$ (Planck Collaboration XVI: Ade, P. A. R. et al., 2013). Em particular, na curva com $\alpha=0, \Omega_{k}=0$, recuperamos o parâmetro de Hubble previsto pelo modelo de Einstein-de Sitter. Obtidas as previsões teóricas do modelo acerca do parâmetro de Hubble, poderemos agora vincular os parâmetros livres do modelo com os dados observacionais.

\subsection{Vínculos Observacionais}

Nesta seção, discutimos os vínculos observacionais sobre os parâmetros livres $\alpha$ e $\Omega_{k}$ do modelo com criação de matéria. Consideramos diferentes amostras observacionais relacionadas ao background cosmológico, conforme discutido a seguir. 

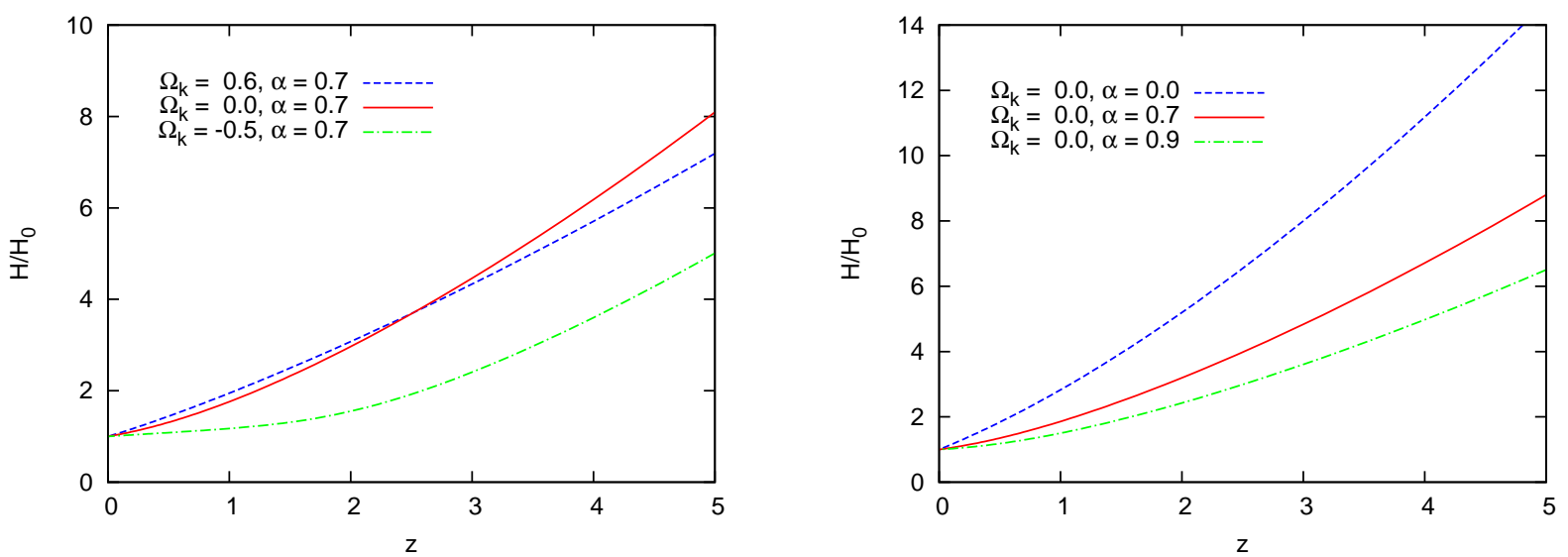

Figura 7.1: Soluções numéricas de parâmetro de Hubble normalizado $H / H_{0}$ em função do redshift z e suas dependências com os parâmetros livres $\alpha$ e $\Omega_{k}$. Esquerda: Evolução de $H(z) / H_{0} \operatorname{com} \alpha=0.7 \mathrm{e}$ $\Omega_{k}=0$ (em vermelho), $\Omega_{k}=0.6$ (em azul) e $\Omega_{k}=-0.5$ (em verde). Direita: Evolução de $H(z) / H_{0} \mathrm{com}$ $\Omega_{k}=0$ e $\alpha=0$ (em azul), $\alpha=0.7$ (em vermelho) e $\alpha=0.9$ (em verde).

Primeiramente, realizamos um teste estatístico de $\chi^{2}$ considerando os dados do parâmetro de Hubble, reunidos em Farooq e Ratra (2013), com 28 pontos de H(z). Adicionalmente, usamos a recente compilação com 580 dados de módulo de distância derivados de observações de SNe Ia da compilação Union 2.1 (Suzuki et al., 2012).

\subsubsection{Limites obtidos através do Parâmetro de Hubble}

Discutimos aqui os vínculos obtidos de dados do parâmetro de Hubble em função de redshift. O comportamento do parâmetro de Hubble é uma dos parâmetros mais básicos de qualquer modelo cosmológico. Em muitos casos, as medidas desse parâmetro dependem apenas de hipóteses astrofísicas, não estando diretamente ligadas a nenhum modelo cosmológico subjacente. Entretanto, por não ser um observável direto, medidas de H(z) não são obtidas de forma simples.

Atualmente, existem na literatura diferentes métodos de obtenção de dados de $H(z)$, entre eles, pela detecção do pico de oscilações acústicas de bárions (Blake et al., 2012; Busca et al., 2013), através da função de correlação de galáxias vermelhas luminosas (Red Luminous Galaxies -LRG) (Chuang e Wang, 2013) ou ainda por meio de medidas de "cronômetros cósmicos" como, por exemplo, a idade diferencial de galáxias (Simon et al., 2005; Stern et al.. 2010: Moresco et al..|2012). Consideramos aqui a compilacão em Farooq e Ratra (2013), sendo uma das compilações mais completas, com 28 dados derivados de medidas observacionais (Tabela 7.1). 
Utilizando os dados de $H(z)$ compilados por Farooq e Ratra (2013), realizamos um teste estatístico de $\chi^{2}$

$$
\chi_{H}^{2}=\sum_{i=1}^{28} \frac{\left[H\left(z_{i} \mid \mathbf{s}\right)-H_{i}\right]^{2}}{\sigma_{H i}^{2}},
$$

onde $H_{i}$ é o valor medido do parâmetro de Hubble no redshift $z_{i}$, com incerteza $\sigma_{H i}$ e $H\left(z_{i} \mid \mathbf{s}\right)$ é obtido numericamente da equação (7.7). Haja vista que o parâmetro de H(z) depende da constante de Hubble através da relação $H(z)=H_{0} E(z)$, eliminamos essa dependência através de uma marginalização analítica sobre $H_{0}$, levando a $\tilde{\chi}_{H}^{2}$ (c.f. apêndice $\mathrm{A}$ ):

$$
\tilde{\chi}_{H}^{2}=C-\frac{B^{2}}{A}
$$

onde

$$
\begin{aligned}
A & \equiv \sum_{i=1}^{28} \frac{E\left(z_{i} \mid \mathbf{s}\right)^{2}}{\sigma_{H i}^{2}} \\
B & \equiv \sum_{i=1}^{28} \frac{E\left(z_{i} \mid \mathbf{s}\right) H_{i}}{\sigma_{H i}^{2}}
\end{aligned}
$$

$\mathrm{e}$

$$
C \equiv \sum_{i=1}^{28} \frac{H_{i}^{2}}{\sigma_{H i}^{2}} .
$$

Os resultados desta análise são apresentados ao lado esquerdo da figura 7.2. Conforme pode se observar, os limites obtidos de dados de $\mathrm{H}(\mathrm{z})$ não impõem vínculos muito restritivos sobre os parâmetros $\alpha$ e $\Omega_{k}$. O valor mínimo de $\chi^{2}$ encontrado foi $\chi_{\min }^{2}=16.269$, levando a um valor de $\chi^{2}$ reduzido muito baixo, $\chi_{\nu}^{2}=0.626$, para 26 graus de liberdade. Os valores de melhor ajuste foram $\alpha=0.791_{-0.085}^{+0.180}$ e $\Omega_{k}=0.04_{-0.40}^{+0.46}$. O valor baixo do $\chi_{\nu}^{2}$ é provavelmente devido a superestimativa das incertezas na amostra de $H(z)$, conforme discutido em Farooq e Ratra (2013).

Complementarmente, ao invés de tomar o caso plano do modelo, assumimos um prior sobre a sua curvatura. Os vínculos provenientes da radiação cósmica de fundo, indicam $\Omega_{k}=-0.037_{-0.049}^{+0.043}$ com $95 \%$ de nível de confiança, no contexto do modelo $\Lambda$ CDM. Considerando-se esse vínculo e utilizando o processo de simetrizacão (Planck Collaboration XVI: Ade, P. A. R. et al., 2013; D’Agostini, 2004), utilizamos como prior para o parâmetro de densidade de curvatura $\Omega_{k} \pm \sigma_{\Omega_{k}}=0.043_{-0.046}^{+0.046}$ - daqui para frente, referido como "CMB prior". Os vínculos sobre os parâmetros livres obtidos são melhorados significantemente, 
conforme é possível verificar ao lado direito da figura 7.2. Para um $\chi_{\min }^{2}=16.344$, encontramos como valor de melhor ajuste $\alpha=0.775$ e $\Omega_{k}=-0.041$ com $64.3 \%, 95.4 \%$ e $99.7 \%$ de nível de confiança, respectivamente.

Na próxima seção, discutimos como os dados provenientes de medidas de SN Ia restringem os parâmetros do modelo.

\subsubsection{Limites obtidos através de Supernovas do Tipo Ia}

O módulo de distância de uma supernova em um dado redshift z pode ser calculado através da expressão

$$
\mu(z \mid \mathbf{s})=m-M=5 \log D_{L}+25,
$$

onde $\mathrm{m}$ e $\mathrm{M}$ são as magnitudes aparente e absoluta, respectivamente, $\mathbf{s}=\left(H_{0}, \alpha, \Omega_{k}\right)$ é o conjunto de parâmetros livres do modelo e $D_{L}$ é a distância de luminosidade, medida em Megaparsecs.

Usualmente, a distância de luminosidade é computada através da expressão (2.45). Entretanto, no caso geral do modelo aqui apresentado, não é possível obter uma função analítica para o parâmetro de Hubble. Assim, a distância de luminosidade pode ser mais facilmente obtida através de uma equação diferencial.

Definimos a distância comóvel $D$ adimensional em função da distância de luminosidade, tal que:

$$
D=\frac{H_{0}}{(1+z) c} D_{L}
$$

A distância comóvel se relaciona com o parâmetro de Hubble $H(z)$ por meio da expressão (Clarkson et al., 2008):

$$
\left(\frac{H}{H_{0}}\right)^{2} \equiv y=\frac{1+\Omega_{k} D^{2}}{D^{\prime 2}},
$$

onde cada sinal (') representa uma derivada em relação ao redshift z.

A equação diferencial que rege o comportamento de $D$ é obtida aplicando a relação (7.21) na equação (7.8): 


$$
\begin{aligned}
D^{\prime \prime} & =\frac{\Omega_{k} D D^{\prime 2}}{1+\Omega_{k} D^{2}}-\frac{3 D^{\prime}}{2(1+z)}+\frac{D^{\prime 3}}{2\left(1+\Omega_{k} D^{2}\right)}\left[\frac{3 \alpha}{1+z}+\Omega_{k}(1+z)\left(1-\frac{3 \alpha D^{\prime 2}}{1+\Omega_{k} D^{2}}\right)\right]- \\
& -\frac{3 \alpha \Omega_{b}(1+z)^{2} D^{\prime 5}}{2\left(1+\Omega_{k} D^{2}\right)^{2}}
\end{aligned}
$$

onde a equação (7.22) é resolvida numericamente, considerando-se as condições iniciais $D(z=0)=0$ e $D^{\prime}(z=0)=1$.

Os vínculos sobre os parâmetros livres do modelo foram obtidos considerando-se a compilação Union 2.1, com 580 dados de SNe Ia (Suzuki et al., 2012). Os valores de s que fornecem melhor ajuste foram estimados por meio de um teste estatístico de $\chi^{2}$, com marginalização sobre o parâmetro $H_{0}$

$$
\chi_{S N}^{2}=\sum_{i=1}^{580} \frac{\left[\mu^{i}\left(z_{i} \mid \mathbf{s}\right)-\mu_{o}^{i}\right]^{2}}{\sigma_{S N i}^{2}},
$$

onde $\mu^{i}(z \mid \mathbf{s})$ é o módulo de distância previsto pelo modelo, $\mu_{o}^{i} z$ é o módulo corrigido de uma SN Ia com redshift $z_{i}$ e com incerteza $\sigma_{i}$, para os 580 pontos da amostra considerada. Assim como na análise com dados de $\mathrm{H}(\mathrm{z})$, nos resultados obtidos aqui aplicamos uma marginalização sobre o parâmetro $H_{0}$

No lado esquerdo da figura (7.2), apresentamos o espaço de parâmetros $\alpha-\Omega_{k}$. Os contornos em verde delimitam os vínculos obtidos pela análise de SN Ia em 68.3\%, 95.4\% e $99.7 \%$ de nível de confiança, respectivamente. Os valores de melhor ajuste obtidos foram $\alpha=0.788_{-0.086}^{+0.230} \Omega_{k}=0.04_{-0.35}^{+0.39} \chi_{\nu}^{2}=0.974$ para 578 graus de liberdade.

\subsubsection{Limites Combinados}

A fim de obter vínculos mais restritivos, combinamos as amostras de dados apresentadas nas seções anteriores. Em nossa análise, minimizamos a função $\chi_{T}^{2}=\chi_{H}^{2}+\chi_{S N}^{2}$ e, complementarmente, discutimos como o prior sobre $\Omega_{k}$ afeta os vínculos combinados.

No lado esquerdo da figura 7.2, as curvas em vermelho delimitam as regiões com $68.3 \%$, 95.4\% e 99.7\% de nível de confiança obtidas da análise conjunta de H(z) e SNe Ia. Notase que os vínculos obtidos da análise de SN Ia (curvas verdes) são razoavelmente mais restritivos. Os valores de melhor ajuste obtidos nesse caso foram $\alpha=0.792_{-0.061-0.093}^{+0.110+0.260}$, $\Omega_{k}=0.05_{-0.24-0.40}^{+0.25+0.42}$, para $\chi_{n u}^{2}=0.956$ e com $68.3 \%$ e $95.4 \%$ de confiança estatística, representando um bom ajuste do modelo aos dados. 

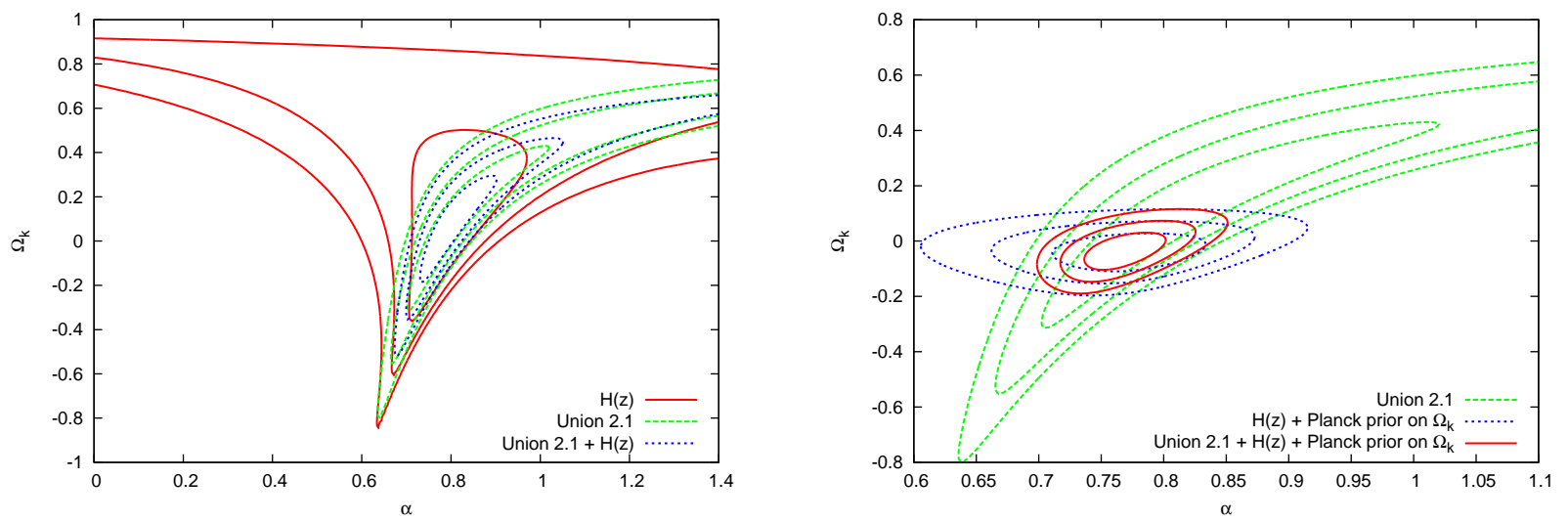

Figura 7.2: Plano $\alpha-\Omega_{k}$ para o modelo CCDM. Esquerda: Vínculos obtidos através da análise de 580 dados de SN Ia do catálogo Union 2.1 (Suzuki et al., 2012) e de 28 dados de $H(z)$ compilados em Farooq e Ratra (2013) . Os contornos delimitam os níveis de confiança estatística de 68.3\%, 95.4\% e 99.7 \%. Direita: Vínculos obtidos considerando-se a combinação de dados de SN Ia, H(z) e um prior sobre o parâmetro de curvatura $\Omega_{k}$ (Planck Collaboration XVI: Ade. P. A. R. et al., 2013) para 68.3\%, 95.4\% e $99.7 \%$ de confiança estatística.

Ao invés de obter vínculos para o caso plano, adicionamos como prior derivado da análise de Planck Collaboration XVI: Ade, P. A. R. et al. (2013), $\Omega_{k} \pm \sigma_{\Omega_{k}}=0.043_{-0.046}^{+0.046}$, com $95 \%$ de nível de confiança. No lado direito da figura 7.2, são mostradas em vermelho as regiões com $68.3 \%, 95.4 \%$ e $99.7 \%$ de nível de confiança, para a combinação de dados de $\mathrm{H}(\mathrm{z})$, SNe Ia e o prior sobre $\Omega_{k}$. Os valores de melhor ajuste obtidos neste caso, foram $\alpha=0.768_{-0.031-0.051-0.069}^{+0.034+0.058+0.084}, \Omega_{k}=-0.036_{-0.068-0.110-0.150}^{+0.066+0.110+0.150}$ para o valor reduzido de $\chi^{2}$, $\chi_{\nu}^{2}=0.957$.

Na figura 7.3, são mostradas as curvas de $H(z)$ para alguns valores selecionados de $\Omega_{k}$, nos cenários CCDM e $\Lambda$ CDM. Em cada curva, fixamos $\alpha=\Omega_{\Lambda}=0.792$, o valor de melhor ajuste encontrado da análise de SN Ia e $H(z)$. Utilizamos o valor de $H_{0}=$ $72.0 \pm 3.0 \mathrm{~km} \mathrm{~s}^{-1} \mathrm{Mpc}^{-1}$, com cada região colorida correspondendo a uma variação de $1 \sigma$ em torno do valor de melhor ajuste. Observamos que, se o Universo é fechado, as diferenças entre as previsões dos modelos CCDM e $\Lambda$ CDM só são significativas em altos redshifts. Por outro lado, para um Universo aberto, as previsões de cada modelo se diferem mais, mesmo em redshifts intermediários.

Todavia, quando utilizamos um prior sobre o parâmetro $\Omega_{k}$ obtido de medidas de CMB (Planck Collaboration XVI: Ade, P. A. R. et al., 2013), determinamos com 95\% de confiança estatística um limite de $-0.104<\Omega_{k}<0.030$. Portanto, os limites observacionais atuais ainda não são capazes de gerar vínculos suficientemente restritivos a ponto de 


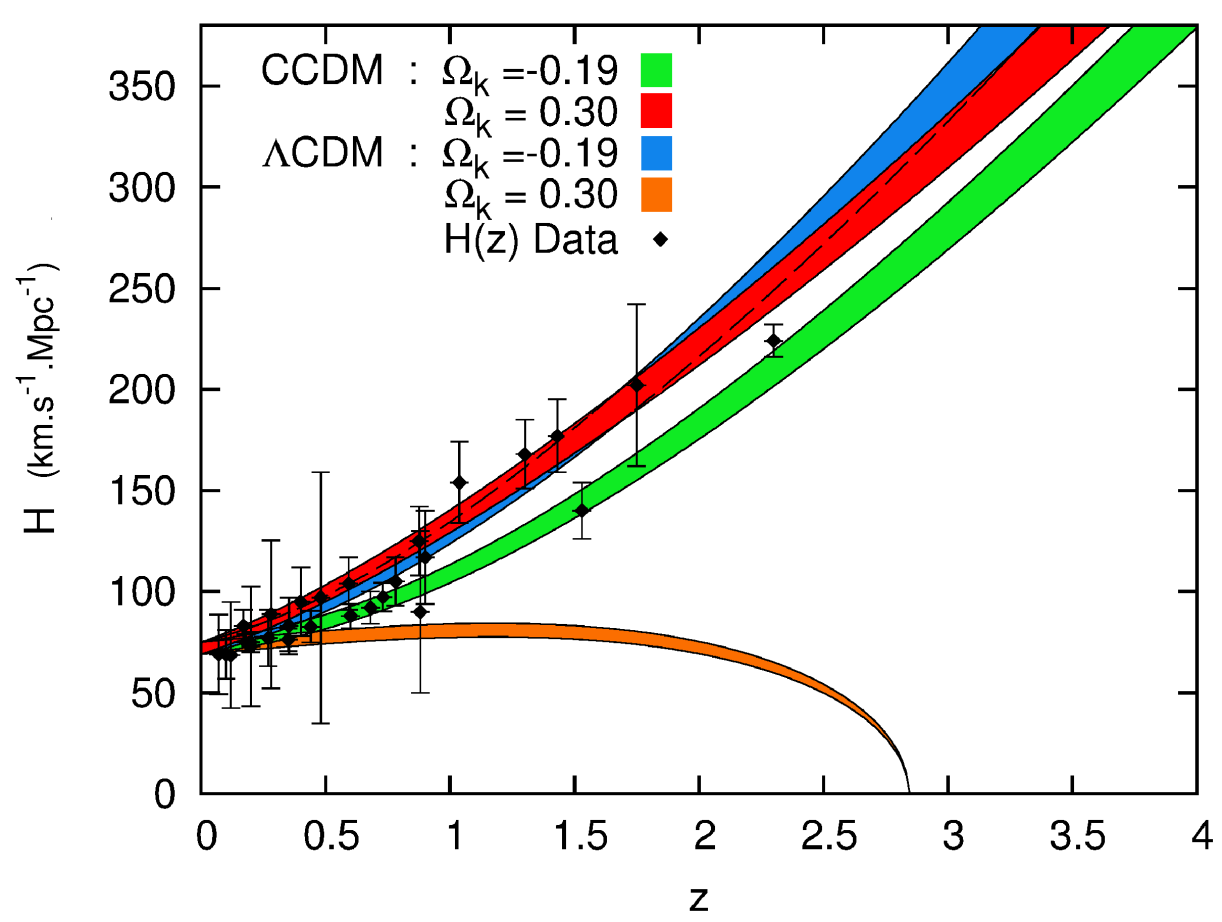

Figura 7.3: Soluções numéricas de $H$ em função do redshift $z$ nos modelos CCDM e $\Lambda$ CDM e suas dependências com os parâmetros livres $\alpha$ e $\Omega_{k}$. Cada região colorida corresponde a uma solução numérica para um conjunto de valores $\left(\alpha, \Omega_{k}\right)$ e à variação de $H_{0}$ em um $1 \sigma$ de incerteza $\left(H_{0}=\right.$ $\left.72.0 \pm 3.0 \mathrm{~km} \mathrm{~s}^{-1} \mathrm{Mpc}^{-1}\right)$.

distinguir entre ambos os modelos.

Os resultados finais de nossa análise podem ser consultados na tabela 7.2 .

\subsection{Discussões}

Assumindo a validade da Teoria da Relatividade Geral, as observações de magnitudes de SNe Ia distantes são geralmente interpretadas como a expansão acelerada do universo. Dentro da classe de modelos homogêneos e isotrópicos na TRG , o ingrediente fundamental para a aceleração do universo é um termo de pressão negativa suficientemente grande para contrabalancear a gravidade dos termos de densidade. Nos capítulos anteriores, discutimos propostas envolvendo o fenômeno de criação gravitacional de partículas (em particular no modelo de Lima, Jesus \& Oliveira) e como a pressão negativa gerada nestes modelos podem explicar as observações cosmológicas.

Conforme vem sendo discutido na literatura, o modelo LJO não pode ser distinguido do modelo $\Lambda$ CDM em nível de background (Lima et al., 2010) e em nível das perturbações 
lineares (Ramos et al., 2014). A essa situação foi dado o nome de "degenerescência escura" (Ramos et al., 2014) em que, em princípio, não seria possível determinar se o presente estado de aceleração do Universo tem origem nas contribuições quânticas do vácuo ( $\Lambda \mathrm{CDM})$ ou no processo de criação de matéria escura fria (CCDM).

Neste capítulo discutimos uma nova proposta cosmológica dentro do cenário com criação de matéria escura. Mostramos que o modelo cuja taxa de criação inversamente proporcional ao parâmetro de Hubble é capaz de descrever a dinâmica de universo com background acelerado, sem a necessidade de um fluido "escuro" adicionalmente à matéria escura (Jesus e Andrade-Oliveira, F., 2015). Esse modelo possui a mesma taxa de criação que o modelo em Graef et al. (2014). Entretanto, nessa análise não foram consideradas as componentes bariônica e de curvatura, estando o modelo de Graef et al. (2014) dentro dos limites de validade do modelo LJO.

No modelo CCDM proposto aqui, a conservação de bárions não pode ser combinada com a conservação do termo de matéria escura, como no caso do modelo LJO. Essa característica implica que a existência de uma componente bariônica já seja condição para a quebra de degenerescência entre os modelos. Em termos quantitativos, considerando-se o parâmetro de densidade de bárions hoje como $\Omega_{b} \sim 0.05$, a diferença relativa entre os parâmetros de Hubble previstos pelos modelos CCDM e $\Lambda$ CDM é da ordem de 10\%, para $z \gtrsim 3$.

Cabe ressaltar que, diferentemente de outros cenários de matéria escura unificada como no caso do gás de Chaplygin generalizado, o modelo apresentado aqui não tem como limite o modelo padrão $\Lambda$ CDM (Kamenshchik et al., 2001; Bilic et al., 2002; Bento et al., 2002; Fabris et al., 2002). Por exemplo, em Wang et al. (2013), foi mostrado que o modelo de gás de Chaplygin modificado só é capaz de descrever a combinação de dados de SN Ia, RCF e estruturas em larga escala, em um limite extremamente pequeno em torno do modelo padrão. Como esse limite não existe no modelo estudado aqui, esperamos que dados mais restritivos eventualmente sejam capazes de distinguir entre ambos os cenários.

Investigamos ainda como os dados do parâmetro de Hubble e Supernovas Tipo Ia vinculam os parâmetro do modelo CCDM. Para isto, realizamos o teste de $\chi^{2}$ utilizando 28 dados de H(z) reunidos em Farooq e Ratra (2013) e a amostra Union 2.1 (Suzuki et al., 2012), contendo os dados de 580 SN Ia. Os vínculos sobre os parâmetros livres foram obtidos marginalizando a constante de Hubble, $H_{0}$. Da combinação de dados de H(z) e SNe Ia, os valores vinculados foram $\alpha=0.792_{-0.061-0.093}^{+0.110+0.260}, \Omega_{k}=0.05_{-0.24-0.40}^{+0.25+0.42}$, com valor de $\chi^{2}$ re- 
duzido, $\chi_{\nu}^{2}=0.956$, favorecendo um universo com uma pequena curvatura positiva. Adicionalmente, consideramos os limites provenientes da RCF como um prior sobre o parâmetro de curvatura. Os vínculos obtidos nessa situação foram $\alpha=0.768_{-0.031-0.051-0.069}^{+0.034+0.058+0.084} \mathrm{e}$ $\Omega_{k}=0.036_{-0.068-0.110-0.150}^{+0.066+0.110+0.150}$, com $\chi_{\nu}^{2}=0.957$.

Em suma, o modelo CCDM apresentado é capaz de fornecer um bom ajustes para as observações cosmológicas em background, sem a necessidade de um fluido escuro adicional à matéria escura. Os dados de $\mathrm{H}(\mathrm{z})$ e SNe Ia favorecerem um universo com pequena quantidade de curvatura, mas compatível com um modelo plano de universo. Permanece portanto a questão de se a curvatura espacial é suficiente para que seja possível a distinção entre as diferentes propostas.

O próximo passo é compreender como a matéria se aglomera neste modelo. Testes estatísticos futuros envolvendo os parâmetros do modelo perturbado deverão ser realizadas a fim de investigar se o modelo é capaz de ajustar os dados relacionados à formação de estruturas do universo e se as distinções entre as previsões de ambos os modelos se acentuam. 


\begin{tabular}{|c|c|c|c|}
\hline$z$ & $\begin{array}{c}H(z) \\
\left(k m s^{-1} M p c^{-1}\right)\end{array}$ & $\begin{array}{c}\sigma_{H} \\
\left(k m s^{-1} M p c^{-1}\right)\end{array}$ & Referência \\
\hline 0.070 & 69 & 19.6 & Zhang et al. (2014) \\
\hline 0.100 & 69 & 12 & Simon et al. (2005) \\
\hline 0.120 & 68.6 & 26.2 & Zhang et al. (2014) \\
\hline 0.170 & 83 & 8 & $\underline{\text { Simon et al. }(2005)}$ \\
\hline 0.179 & 75 & 4 & Moresco et al. (2012) \\
\hline 0.199 & 75 & 5 & Moresco et al. (2012) \\
\hline 0.200 & 72.9 & 29.6 & Zhang et al. (2014) \\
\hline 0.270 & 77 & 14 & Simon et al. $(2005)$ \\
\hline 0.280 & 88.8 & 36.6 & Zhang et al. (2014) \\
\hline 0.350 & 76.3 & 5.6 & Chuang e Wang (2013) \\
\hline 0.352 & 83 & 14 & Moresco et al. (2012) \\
\hline 0.400 & 95 & 17 & $\underline{\text { Simon et al. }(2005)}$ \\
\hline 0.440 & 82.6 & 7.8 & Blake et al. (2012) \\
\hline 0.480 & 97 & 62 & Stern et al. (2010) \\
\hline 0.593 & 104 & 13 & Moresco et al. (2012) \\
\hline 0.600 & 87.9 & 6.1 & Blake et al. (2012) \\
\hline 0.680 & 92 & 8 & Moresco et al. (2012) \\
\hline 0.730 & 97.3 & 7.0 & Blake et al. (2012) \\
\hline 0.781 & 105 & 12 & Moresco et al. (2012) \\
\hline 0.875 & 125 & 17 & Moresco et al. (2012) \\
\hline 0.880 & 90 & 40 & Stern et al. (2010) \\
\hline 0.900 & 117 & 23 & Simon et al. $(2005)$ \\
\hline 1.037 & 154 & 20 & Moresco et al. (2012) \\
\hline 1.300 & 168 & 17 & Simon et al. $(2005)$ \\
\hline 1.430 & 177 & 18 & Simon et al. $(2005)$ \\
\hline 1.530 & 140 & 14 & Simon et al. $(2005)$ \\
\hline 1.750 & 202 & 40 & Simon et al. $(2005)$ \\
\hline 2.300 & 224 & 8 & Busca et al. (2013) \\
\hline
\end{tabular}

Tabela 7.1 - Dados de $H(z)$ em função do redshift z e suas incertezas. Na coluna mais à direita da tabela acima, são apresentadas as referências de cada um dos dados de $H(z)$, conforme a compilação realizada por Farooq e Ratra (2013). 


\begin{tabular}{|c|c|c|c|}
\hline Dados & $\alpha$ & $\Omega_{k}$ & $\chi_{\nu}^{2}$ \\
\hline \hline$H(z)$ & $0.791_{-0.085}^{+0.180}$ & $0.04_{-0.40}^{+0.46}$ & 0.651 \\
\hline $\mathrm{SN}$ & $0.788_{-0.086}^{+0.230}$ & $0.04_{-0.35}^{+0.39}$ & 0.974 \\
\hline $\mathrm{SN}+H(z)$ & $0.792_{-0.061}^{+0.110}$ & $0.05_{-0.24}^{+0.25}$ & 0.956 \\
\hline$H(z)+\mathrm{CMB}$ prior & $0.775_{-0.064}^{+0.059}$ & $-0.041_{-0.069}^{+0.069}$ & 0.654 \\
\hline $\mathrm{SN}+H(z)+\mathrm{CMB}$ prior & $0.768_{-0.031}^{+0.034}$ & $-0.036_{-0.068}^{+0.066}$ & 0.957 \\
\hline
\end{tabular}

Tabela 7.2 - Resultados da análise estatística para os parâmetros livres do modelo em diferentes combinações de dados. Os limites apresentados correspondem a $68.3 \%$ de nível de confiança estatística. 
Capítulo 8

\section{Modelo com Decaimento do Vácuo e Descrição por}

\section{Campos Escalares}

\subsection{Introdução}

Nos modelos investigados nos capítulos anteriores, a criação de partículas é induzida pelo campo gravitacional, tal qual nos processos quânticos (Parker, 1968; Birrell e Davies, 1982; Parker e Vanzella, 2004; Parker, 2012). No entanto, é conhecido que o decaimento devido ao acoplamento com os outros campos de matéria também gera partículas. Tais modelos são conhecidos como modelos de decaimento do vácuo, $\Lambda(t) \mathrm{CDM}$. Discutiremos neste capítulo um modelo de energia escura com decaimento do vácuo, além de sua descrição como um modelo de campo escalar acoplado.

Em modelos $\Lambda(\mathrm{t}) \mathrm{CDM}$, assumimos que a densidade de energia do vácuo satisfaz à equação de estado $p_{v}=-\rho_{v}$, como no caso da constante cosmológica, porém com densidade variável. No contexto da Relatividade Geral, um termo do tipo $\Lambda$ que possa variar no espaço ou no tempo deve estar acoplado a outro componente de forma que o tensor energiamomento total seja conservado. Portanto, modelos com decaimento ou estão ligados a processos de produção ou destruição de partículas ou a uma variação temporal da massa das partículas de matéria escura (Alcaniz e Lima, 2005a). Esse acoplamento é também sugerido pelo fato de que a matéria escura e energia escura possuem a mesma ordem de grandeza. Nos modelos com decaimento, o baixo valor da energia do vácuo e a questão da coincidência cósmica podem ser interpretados como consequência de vivermos em um universo suficientemente velho.

Muitos modelos fenomenológicos vêm sendo propostos dentro do cenário com decaimento do vácuo. Entre eles, podemos citar os modelos em que consideram termos cos- 
mológicos variáveis (i) como uma tentativa de aliviar o problema da constante cosmológica, como por exemplo, Carvalho et al. (1992); Lima e Maia (1994); Lima (1996); Özer e Taha (1986); Freese et al. (1987): Chen e Wu (1990): Pavón (1991): Waga (1993): Overduin e Cooperstock (1998); Cunha e Santos (2004); Shapiro et al. (2005); Elizalde et al. (2005); Grande et al. (2006); Borges et al. (2008); (ii) como uma tentativa de aliviar o problema da coincidência cósmica, entre eles Wang e Meng (2005); Jesus et al. (2008); Costa et al. (2008); Costa e Alcaniz (2010); ou ainda (iii) com o intuito de resolver ambos os problemas, como nos casos dos modelos de Alcaniz e Lima (2005a); Lima et al. (2012, 2013). Recentemente, Salvatelli et al. (2014) mostraram através da análise de dados das anisotropias da radiação cósmica de fundo, supernovas do tipo Ia e distorções no espaço de redshift que um universo sem interação no setor escuro, como no caso do modelo $\Lambda \mathrm{CDM}$, estaria excluído com $99 \%$ de confiança estatística.

Neste capítulo, investigamos aspectos teóricos e observacionais de um modelo $\Lambda$ (t)CDM em que o termo de vácuo evolui como uma série de potências truncada do parâmetro de Hubble (Andrade-Oliveira, F. et al., 2014). Além disso, discutimos como o modelo fenomenológico de energia escura pode ser descrito por um campo escalar acoplado. Na seção 8.2. discutimos aspectos teóricos básicos do modelo com decaimento do vácuo apresentado aqui. Na seção 8.4, discutimos os aspectos termodinâmicos do modelo através da segunda lei da termodinâmica generalizada. Os vínculos observacionais sobre os parâmetros livres do modelo são discutidos na seção 8.5. Seguindo a abordagem proposta por Maia e Lima (2002), descrevemos o modelo de decaimento do vácuo na linguagem da teoria quântica de campos através de um campo escalar acoplado, na seção 8.6. Os resultados obtidos e discussões relevantes são apresentados na seção 8.7 .

\subsection{O Modelo $\Lambda(t)$}

Iniciaremos por considerar um universo homogêneo, isotrópico e espacialmente plano $(K=0)$, descrito através do elemento de linha de Friedman-Lemaître-Robertson-Walker (Weinberg, 1972). Assumindo esse background, as equações de Einstein tomam a forma:

$$
8 \pi G \rho_{f}+\Lambda=3 H^{2}
$$


e

$$
8 \pi G p_{f}-\Lambda=-2 \dot{H}-3 H^{2}
$$

onde $\rho_{f}$ e $p_{f}$ são, respectivamente, as densidades de energia e pressão totais do fluido cósmico, composto por radiação, bárions e matéria escura. Cada sinal de ponto $\left({ }^{*}\right)$ representa uma derivada temporal.

Primeiramente, consideramos aqui $\Lambda(t)$ como uma função de série de potências do parâmetro de Hubble até a segunda ordem:

$$
\Lambda(H)=\lambda+\bar{\sigma} H+3 \beta H^{2}
$$

onde $\lambda$ e $\bar{\sigma}$ são constantes com dimensões de $H^{2}$ e $H$ respectivamente e $\beta$ é uma constante adimensional.

No contexto da cosmologia inflacionária, o termo de vácuo proporcional a $H^{2}$ foi proposto por Carvalho et al. (1992) baseado em argumentos dimensionais. Posteriormente, esse termo foi justificado por Shapiro e Solà (2002); Solà (2011) com argumentos baseados em técnicas de grupos de renormalização. O termo de vácuo proporcional a $H$ foi originalmente discutido em Carneiro et al. (2006) enquanto o interesse em modelos estendidos com o termo de vácuo descrito por uma série de potências truncada foi ressaltado por Basilakos (2009).

Da combinação das equações (8.1) e (8.2) e tomando $p_{f}=(\gamma-1) \rho_{f}$, onde $\gamma$ é o índice adiabático, obtemos:

$$
2 \dot{H}+3 \gamma(1-\beta) H^{2}-\bar{\sigma} \gamma H-\gamma \lambda=0
$$

Da integração da equação anterior, obtemos o parâmetro de Hubble:

$$
H \equiv \frac{\dot{a}}{a}=\frac{1}{3(1-\beta)}\left[\frac{\bar{\sigma}}{2}+\frac{\alpha}{\gamma} \frac{e^{\alpha t}+1}{e^{\alpha t}-1}\right]
$$

onde $\alpha=(\gamma / 2) \sqrt{\bar{\sigma}^{2}+12 \lambda(1-\beta)}$ e $a$ é o fator de escala. Daqui para frente, restringimos nossa análise ao caso $\lambda=0$ uma vez que o fator linear na equação (8.3) já é suficiente para obtermos um modelo que apresente transição de uma fase desacelerada a uma acelerada. Dada tal consideração, o fator de escala toma a forma:

$$
a(t)=C\left(e^{\bar{\sigma} \gamma t / 2}-1\right)^{2 / 3 \gamma(1-\beta)},
$$


onde $C$ é uma constante de integração. Das equações (8.5) e (8.6) obtemos o parâmetro de Hubble em função do fator de escala:

$$
H=\frac{\bar{\sigma}}{3(1-\beta)}\left[1+\left(\frac{C}{a}\right)^{3(1-\beta) / 2}\right]
$$

onde usamos $\gamma=1$. É importante esclarecer que estamos analisando somente o caso em que $\Lambda(t)$ está acoplado com a componente de matéria escura. Naturalmente, em um cenário mais completo, outras constantes de acoplamento devem ser consideradas, a saber, matéria escura $\left(\beta_{d m}\right)$, bárions $\left(\beta_{b}\right)$ e fótons $\left(\beta_{r}\right)$.

Em princípio, isso ocorre porque as constantes de acoplamento com o vácuo não são as mesmas para diferentes canais de decaimento. No modelo aqui discutido, estamos considerando uma interação interna no setor escuro do Universo, de forma que $\beta_{d m} \equiv$ $\beta$ e $\beta_{r}=\beta_{b}=0$. Em particular, isso implica que os modelos de recombinação e de nucleossíntese primordial não são afetados pelo decaimento do vácuo. Nos casos em que escolhas diferentes são consideradas, as constantes de acoplamento deverão ser vinculadas através dos fenômenos citados.

Tomando os valores atuais das equações (8.1), (8.3) e (8.7) é possível mostrar que $C=\Omega_{m} /\left(1-\beta-\Omega_{m}\right)^{2 / 3(1-\beta)}$ e $\bar{\sigma}=3 H_{0}\left(1-\beta-\Omega_{m}\right)$, sendo $\Omega_{m}=8 \pi G \rho_{m, 0} / 3 H_{0}^{2}$. Dessa forma, o parâmetro de Hubble em função do redshift é dado pela expressão:

$$
H=H_{0}\left[1-\frac{\Omega_{m}}{1-\beta}+\frac{\Omega_{m}}{1-\beta}(1+z)^{3(1-\beta) / 2}\right] .
$$

No caso $\beta=0$, a expressão anterior recupera a solução dinâmica para $\Lambda$ obtida por Carneiro et al. (2006).

Utilizando a expressão (8.2), o parâmetro de desaceleração (equação 2.29) pode ser calculado através da expressão:

$$
q(z)=\frac{3}{2} \frac{\Omega_{m}(1+z)^{3(1-\beta) / 2}}{\left[1-\frac{\Omega_{m}}{1-\beta}+\frac{\Omega_{m}}{1-\beta}(1+z)^{3(1-\beta) / 2}\right]}-1 .
$$

Para $z=0$, o valor atual do parâmetro de desaceleração é $q_{0}=\frac{3}{2} \Omega_{m}-1$. Neste caso, o estado da aceleração atual do Universo independe dos valores do parâmetro $\beta$. De acordo com a expressão (8.9), no futuro o parâmetro de desaceleração tende a $q(z \rightarrow-1)=-1$.

O redshift de transição $z_{t}$ é definido como o redshift em que o parâmetro de desaceleração 
muda de sinal. Igualando a equação (8.9) a zero, podemos obter:

$$
z_{t}=\left[\frac{2\left(1-\beta-\Omega_{m}\right)}{(1-3 \beta) \Omega_{m}}\right]^{2 / 3(1-\beta)}-1 .
$$

Para essa classe de modelo $\Lambda(\mathrm{t})$, a idade do universo em um dado redshift , $t(z)$, é determinada por meio da equação:

$$
t(z)=\frac{2}{\bar{\sigma}} \ln \left[1+\frac{1-\beta-\Omega_{m}}{\Omega_{m}}(1+z)^{-3(1-\beta) / 2}\right] .
$$

Portanto, neste modelo de decaimento do vácuo, a idade atual do universo depende dos parâmetros livres do modelo através da relação:

$$
t_{0}=\frac{2 H_{0}^{-1}}{3\left(1-\beta-\Omega_{m}\right)} \ln \left(\frac{1-\beta}{\Omega_{m}}\right) .
$$

As grandezas deduzidas $q_{0}, z_{t}$ e $t_{0}$ são dependentes dos parâmetros livres do modelo. Discutiremos na seção 8.5. como os vínculos observacionais sobre os parâmetros livres determinam seus valores.

\subsection{Criação de Matéria ou Variação de Massa?}

Seguindo as discussões realizadas por Alcaniz e Lima (2005a), vamos investigar algumas características de cenários com decaimento do vácuo. Primeiramente, o comportamento termodinâmico do vácuo pode ser simplificado ao assumirmos que seu potencial químico é nulo e que essa componente se comporta como um condensado que não carrega entropia, assim como na descrição de dois fluidos na termodinâmica de superfluidos (Lima, 1996; Alcaniz e Lima, 2005a). Dessa forma, a descrição termodinâmica é realizada em função dos vetores fluxo de partículas $N^{\mu}=n u^{\mu}$ e fluxo de entropia $S^{\mu}=n \sigma u^{\mu}$, onde $n=N / V$ é a concentração de partículas e $\sigma=S / N$ é a entropia específica (por partícula) das componentes criadas.

No processo de troca de energia entre as componentes de vácuo e de matéria, contudo, não é claro qual a maneira que a matéria criada armazena a energia recebida do decaimento. Uma vez que a densidade de matéria de escura é $\rho \equiv m n$, existem duas possibilidades a serem exploradas (Wang e Meng, 2005; Alcaniz e Lima, 2005a): (i) A energia do decaimento do vácuo cria partículas, de modo que à equação descrevendo a evolução da concentração $n$ é adicionado um termo de fonte, enquanto a massa das partículas permanece constante, 
ou (ii) a massa das partículas de matéria escura é uma quantidade dependente do tempo, enquanto o número total de partículas $\left(N=n a^{3}\right)$ permanece constante.

No primeiro caso, um termo de fonte de partículas $\psi$ deve ser adicionado à equação de concentração, i.e.,

$$
\dot{n}+3 \frac{\dot{a}}{a} n=\psi=n \Gamma
$$

na qual $\psi>0$ representa a criação, $\psi<0$ representa a destruição e $\Gamma$ é a taxa de criação de partículas.

A transferência de energia do termo de vácuo $\rho_{\Lambda}=\Lambda(t) / 8 \pi G$ para a densidade de matéria escura fria $\rho=m n$ é quantificada pela expressão:

$$
\dot{\rho}+3 \frac{\dot{a}}{a} \rho=-\frac{\dot{\Lambda}(t)}{8 \pi G}
$$

Uma vez que neste caso assumimos que a massa $m$ das partículas criadas permanece constante, temos que:

$$
8 \pi G m\left[\dot{n}+3 \frac{\dot{a}}{a} n\right]=-\dot{\Lambda}(t) .
$$

Para o termo de energia de vácuo $\Lambda(t)=\bar{\sigma} H+3 \beta H^{2}$ considerado aqui, pode-se mostrar que

$$
\left(n \dot{a}^{3}\right)=\frac{n_{0}}{2 H_{0}(1-\beta)}\left[\bar{\sigma}(1+\beta) a^{\frac{1+3 \beta}{2}}+6 \beta H_{0} \Omega_{m} a^{-1+3 \beta}\right] \dot{a} .
$$

A equação acima, na ausência do termo $\Lambda(\mathrm{t})$ (representada na condição $\bar{\sigma}=\beta=0$ ), tem como solução:

$$
n(a)=n_{0} a^{-3}
$$

onde $n_{0}$ é a concentração de partículas hoje. No caso $(\bar{\sigma} \neq 0)$ e $(\beta \neq 0)$, a equação (8.16) tem como solução:

$$
n(a)=\frac{n_{0} a^{-3}}{1-\beta}\left[\left(1-\beta-\Omega_{m}\right) a^{\frac{3(1+\beta)}{2}}+\Omega_{m} a^{3 \beta}\right]
$$

onde utilizamos que a concentração de partículas hoje é $n_{0}=\frac{3 H_{0}^{2} \Omega_{m}}{8 \pi G m}$.

No segundo caso, quando a energia do decaimento é armazenada na variação de massa das partículas, a concentração satisfaz à equação:

$$
\dot{n}+3 \frac{\dot{a}}{a} n=0
$$


com a solução $n=n_{0} a^{-3}$, ou ainda, $N=$ constante. Uma vez que partículas de matéria escura não estão sendo criadas, a transferência de energia deve ocorrer através do aumento de suas massas. Utilizando a conservação de energia (equação 8.14), obtemos

$$
\dot{m}=-\frac{\dot{\Lambda}(t)}{8 \pi G n_{0} a^{3}},
$$

cuja solução em termos do fator de escala é dada por:

$$
m(a)=\frac{m_{0}}{1-\beta}\left[\left(1-\beta-\Omega_{m}\right) a^{3(1+\beta)}+\Omega_{m} a^{3 \beta}\right],
$$

onde usamos que o valor atual da massa da partícula de matéria escura é $m_{0}=\frac{3 H_{0}^{2} \Omega_{m}}{8 \pi G n_{0}}$. Essa abordagem conduz aos cenários do tipo VAMP (VAriable-Mass Particles), nos quais a massa da partícula de matéria escura evolui como uma funcão da energia escura (Casas et al., 1992; Anderson e Carroll, 1997; Amendola et al., 2003; França e Rosenfeld, 2004; Leon e Saridakis, 2010).

Naturalmente, a transferência de energia através da criação de partículas parece ser mais razoável. Nesse caso, é possível estabelecer uma equivalência entre os modelos com decaimento do vácuo e os modelos com criacão de matéria, conforme discutido por Graef et al. (2014). Embora a física por trás dos cenários CCDM e $\Lambda(\mathrm{t}) \mathrm{CDM}$ sejam completamente distintas, sob certas condições ambos podem convergir para as mesmas dinâmicas e termodinâmicas.

De maneira geral, a conservação de energia no decaimento do vácuo em uma componente material é dada por:

$$
\dot{\rho}+3 \frac{\dot{a}}{a}(\rho+p)=-\dot{\rho}_{\Lambda}
$$

Tomando o decaimento do vácuo como única fonte de criação de partículas, podemos escrever:

$$
\dot{\rho}_{\Lambda}=-\zeta n \Gamma
$$

$\zeta$ é um parâmetro fenomenológico positivo. Por outro lado, nos processos dentro do cenário CCDM, daqui para frente identificados pelo til ( ), a criação afeta a concentração de partículas da seguinte forma: 


$$
\tilde{N}^{\mu} ;_{\mu}=\dot{\tilde{n}}+3 \frac{\dot{a}}{a} \tilde{n}=\tilde{n} \tilde{\Gamma}
$$

onde $\tilde{\Gamma}$ é a taxa de criacão induzida pelo campo gravitacional (Lima et al.. 1991: Calvão et al., 1992; Lima e Germano, 1992). A pressão de criação pode ser descrita como:

$$
p_{c}=-\tau \frac{\tilde{n} \tilde{\Gamma}}{3 H},
$$

onde $\tau$ é um coeficiente fenomenológico, ligado ao processo de criação.

Da combinação das equações (8.1) e (8.2), obtemos para a relação válida para modelos de decaimento do vácuo em geral (Lima, 1996):

$$
\frac{\ddot{a}}{a}+\frac{3 \omega+1}{2}\left(\frac{\dot{a}^{2}}{a^{2}}+\frac{K}{a^{2}}\right)+\frac{(1+\omega) \Lambda(t)}{2}=0
$$

onde $\omega=\gamma+1$ e o termo $K / a^{2}$ foi inserido para um universo com curvatura arbitrária. De forma análoga, para um modelo CCDM, temos (equações 4.11 e 4.12):

$$
\frac{\ddot{a}}{a}+\frac{3 \omega+1}{2}\left(\frac{\dot{a}^{2}}{a^{2}}+\frac{K}{a^{2}}\right)-\frac{8 \pi G}{2} p_{c}=0,
$$

ou seja, a equivalência dinâmica entre os cenários CCDM e $\Lambda(t)$ CDM se dá através da identificação (Graef et al., 2014)

$$
8 \pi G p_{c}=-(1+\omega) \Lambda(t)
$$

A condição acima é bastante geral, sendo válida para os cenários de energia do vácuo com $\Lambda$ constante ou variável e independentemente da forma fenomenológica do decaimento do vácuo ou da taxa de criação consideradas.

Utilizando a condição "adiabática" de criação $(\dot{\sigma}=0)$, a pressão de criação em termos da taxa $\Gamma$ é dada por (Lima et al., 1991; Calvão et al., 1992):

$$
p_{c}=-\frac{\tilde{\rho}+\tilde{p}}{3 H} \tilde{\Gamma}=-\frac{(1+\omega) \tilde{\rho} \tilde{\Gamma}}{3 H} .
$$

Em função da taxa de criação dos modelos CCDM, a condição de equivalência dinâmica entre os cenários pode ser expressa por

$$
\Lambda(t)=\frac{\tilde{\rho} \tilde{\Gamma}}{3 H}
$$


independentemente da curvatura do universo. As condições (8.25) e (8.30) garantem que ambos os modelos reproduzam a mesma dinâmica cosmológica. Vamos agora discutir quais são as condições que garantam a equivalência termodinâmica.

A termodinâmica básica dos modelos com $\Lambda(t)$ e dos modelos CCDM são temas de diversos trabalhos na literatura, como por exemplo (Lima et al., 1991; Calvão et al., 1992; Lima, 1996; Alcaniz e Lima, 2005a; Graef et al., 2014). Condições de equivalência termodinâmica entre ambos os modelos podem ser estabelecidas através da análise das leis de evolução de temperatura e de entropia específica $(S / N)$ das componentes criadas, conforme originalmente realizado por (Graef et al., 2014).

Assumindo-se que o potencial químico do vácuo seja nulo $\left(\mu_{v}=0\right)$, temos que a entropia específica do vácuo é $\sigma_{v}=0$ (Equação 4.16). Assim, combinando-se o fluxo de entropia $S^{\alpha}=n \sigma u^{\alpha}$ com as expressões (8.13) e (8.23), obtemos (Graef et al., 2014)

$$
\dot{\sigma}+\sigma \Gamma=\frac{\Gamma}{T}(\zeta-\mu)
$$

onde $\mu$ denota o potencial químico das partículas criadas e $T$ a temperatura satisfazendo à lei de evolução (Lima, 1996):

$$
\frac{\dot{T}}{T}=\left(\frac{\partial p}{\partial \rho}\right)_{n} \frac{\dot{n}}{n}-\frac{\Gamma}{T(\partial \rho / \partial T)_{n}}\left[T\left(\frac{\partial p}{\partial T}\right)_{n}+n\left(\frac{\partial \rho}{\partial n}\right)_{T}-\zeta n\right] .
$$

Nos modelos CCDM, em contrapartida, a entropia obedece a

$$
\dot{\tilde{\sigma}}+\tilde{\sigma} \tilde{\Gamma}=\frac{\tilde{\Gamma}}{\tilde{T}}(\tau-\tilde{\mu})
$$

enquanto a lei de evolução da temperatura é dada por (Lima et al., 1991; Calvão et al., 1992):

$$
\frac{\dot{\tilde{T}}}{\tilde{T}}=\left(\frac{\partial \tilde{p}}{\partial \tilde{\rho}}\right)_{\tilde{n}} \frac{\dot{\tilde{n}}}{\tilde{n}}-\frac{\tilde{\Gamma}}{\tilde{T}(\partial \tilde{\rho} / \partial \tilde{T})_{\tilde{n}}}\left[\tilde{T}\left(\frac{\partial \tilde{p}}{\partial \tilde{T}}\right)_{\tilde{n}}+\tilde{n}\left(\frac{\partial \tilde{\rho}}{\partial \tilde{n}}\right)_{\tilde{T}}-\tau \tilde{n}\right] .
$$

Claramente, da comparação das equações (8.32) e (8.34), vemos que a condição $\tau=$ $\zeta$ garante que ambos os modelos sejam termodinamicamente equivalentes. Além disso, essa condição quando satisfeita implica que $\dot{\rho}_{\Lambda}=3 H p_{c}$, em harmonia com a condição de equivalência dinâmica mostrada anteriormente. Considerando ainda o processo de criação "adiabático", onde a entropia por partícula permanece constante, a equação (8.25) implica que $\zeta=\tau=\frac{\rho+p}{n}$ (c.f. equação 4.20) (Graef et al., 2014). 


\subsection{Segunda Lei da Termodinâmica Generalizada}

Desde os primeiros estudos em termodinâmica de buracos negros nos anos 1970 (Bekenstein, 1972, 1973; Hawking, 1975), vêm sendo levantadas questões sobre a natureza da ligação entre a gravitação e a termodinâmica. A possível ligação entre teorias foi ilustrada por Jacobson (1995) na questão: “como a TRG clássica sabe que a área do horizonte se transforma em uma forma de entropia e que a gravidade superficial é uma temperatura?".

Nesse espírito, alguns trabalhos na literatura mostraram ser possível utilizar as relações termodinâmicas fundamentais para derivar as equações de Friedmann para um universo acelerado (Gibbons e Hawking, 1977; Pavon, 1990; Radicella e Pavón, 2012). Por exemplo, através da identificação

$$
T \propto \frac{1}{2 \pi r_{A}}, \quad S \propto \frac{A}{4},
$$

onde $r_{A}$ e $A$ são o raio e a área do horizonte aparente, Cai e Kim (2005) tiveram êxito em obter as equações de Friedmann dentro da TRG e das teorias gravitacionais de GaussBonnet e Lovelock. Ainda no contexto cosmológico, Custodio e Horvath (2003) mostraram uma conexão entre a termodinâmica de buracos negros e a termodinâmica de horizontes cosmológicos, ao demonstrar que o princípio holográfico é capaz de impor limites sobre a formação de buracos negros primordiais.

É portanto natural esperar que o universo como um todo deva obedecer às leis fundamentais da termodinâmica. Seguindo a metodologia empregada por Mimoso e Pavón (2013), abordamos aqui algumas propriedades termodinâmicas do modelo com decaimento do vácuo. Discutimos a aplicação da segunda lei generalizada da termodinâmica (SLG) ao modelo e como seus parâmetros livres são termodinamicamente restringidos.

Para que o modelo cosmológico tenha consistência do ponto de vista termodinâmico, é necessário que sua entropia total nunca decresça $(\dot{S} \geq 0)$ e que seja uma função côncava, $\ddot{S}<0$ (Callen, 1985; Cai e Kim, 2005; Cai et al., 2009). Esta última condição garante que, no caso em que haja aumento de entropia do sistema, essa mudança de estado leve o sistema a um novo estado de equilíbrio (Mimoso e Pavón, 2013).

Estamos considerando a entropia total $S$ como a soma da entropia do horizonte $S_{H}$ e da entropia da matéria (sem pressão) $S_{m}$. A entropia do horizonte aparente é dada por (Pavón e Radicella, 2013): 


$$
S_{H}=\frac{k_{B} \mathcal{A}}{l_{p l}^{2}},
$$

onde $\mathcal{A}=4 \pi \tilde{r}_{\Delta}^{2}$ é a área do horizonte aparente, $\tilde{r}_{\mathcal{A}}=c / \sqrt{H^{2}+k a^{-2}}$ é o raio do horizonte aparente (Bak e Rey, 2000),$k_{B}$ é a constante de Boltzmann e $l_{p l}$ é o comprimento de Planck. Considerando o modelo de universo plano, temos que $\tilde{r}_{\mathcal{A}}=\mathrm{cH}^{-1}$.

Por outro lado, a contribuição à entropia dada pelo termo de matéria pode ser estimada assumindo que cada partícula dentro do horizonte aparente contribui com uma unidade de $k_{B}$ para a entropia. Portanto, a entropia total das partículas dentro do horizonte é $N k_{B}$ (Mimoso e Pavón, 2013), ou seja,

$$
S_{m}=k_{B} \frac{4 \pi \tilde{r}_{A}^{3}}{3} \frac{\rho}{m_{d}}
$$

sendo $\rho$, a densidade de matéria sem pressão e $m_{d}$ a massa de suas partículas.

Exceto em altos redshifts, a entropia da matéria é desprezível em comparação com a entropia ligada ao horizonte aparente. Explicitamente, a razão entre as entropias é dada por

$$
\frac{S_{m}}{S_{H}}=\frac{4 \pi}{3} \frac{c l_{p l}^{2}}{m_{d m}} \frac{\rho}{H},
$$

e, considerando-se que $\rho / H=3 H_{0} \Omega_{m} a^{-3(1-\beta) / 2} /(8 \pi G)$, temos que

$$
\frac{S_{m}}{S_{H}}=\frac{4 \pi}{3} \frac{c l_{p l}^{2}}{m_{d m}} \frac{3 H_{0} \Omega_{m}}{8 \pi G} \frac{1}{a^{3(1-\beta) / 2}},
$$

cuja razão é monotonicamente decrescente e com limite

$$
\lim _{a \rightarrow \infty} \frac{S_{m}}{S_{H}}=0 .
$$

Em outras palavras, isso significa que o termo $S_{m}$ tem papel dominante sobre o termo $S_{H}$ no início do Universo. Porém, após o momento em que o fator de escala atinge o valor de $a \sim\left[l p^{2} / m_{d}\right]^{2 / 3(1-\beta)}$, o termo $S_{m}$ é rapidamente dominado pela entropia do horizonte aparente. Uma vez que o modelo considerado aqui abrange as fases mais tardias do Universo (era da matéria e era da energia escura), podemos simplesmente considerar que o comportamento da entropia é regido pela evolução do termo $S_{H}$.

Partindo das considerações expostas, estamos aptos a avançar na investigação sobre o comportamento do modelo sob a luz da segunda lei da termodinâmica. Derivando a expressão (8.36) em relação ao tempo, obtemos 


$$
\dot{S}=-\frac{3 \pi k_{B} c^{2}}{l_{p l}^{2}} \frac{\dot{H}}{H^{3}}
$$

que é sempre positiva ou nula, uma vez que $\dot{H} \leq 0$ e $H>0$.

Portanto, a entropia do universo sempre cresce dentro de um tempo finito. Do ponto de vista termodinâmico, a expansão cosmológica pode ser interpretada como um processo termodinâmico irreversível.

Utilizando a relação:

$$
\dot{H}=-\frac{3}{2} H_{0} \Omega_{m} H a^{-3(1-\beta) / 2},
$$

reescrevemos a primeira derivada da entropia como:

$$
\dot{S}=-\frac{3 \pi k_{B} c^{2} H_{0} \Omega_{m}}{l_{p l}^{2}} \frac{a^{-3(1-\beta) / 2}}{H^{2}} .
$$

Derivando a equação (8.42) em relação ao tempo, obtemos:

$$
\ddot{S}=-\frac{3}{2} \frac{\pi k_{B} c^{2} \Omega_{m} H_{0} a^{3(1-\beta) / 2}}{l_{p l}^{2} H^{3}}\left[\frac{3 H^{2}}{2}+2 \dot{H}\right],
$$

ou, utilizando a relação (8.41), temos

$$
\ddot{S}=-\frac{3 \pi k_{B} c^{2} \Omega_{m} H_{0} a^{-3(1-\beta) / 2}}{l_{p l}^{2} H^{2}}\left[\frac{3}{2} H-3 \Omega_{m} H_{0} a^{-3(1-\beta) / 2}\right] .
$$

Das expressões (8.44) e (8.1) , concluímos que $\ddot{S}<0$ quando $a_{S}>\left[\Omega_{m} /(1-\beta-\right.$ $\left.\left.\Omega_{m}\right)\right]^{2 / 3(1-\beta)}$. A entropia da densidade de energia do vácuo começa a dominar em $a=$ $\left[(1-2 \beta) \Omega_{m} /\left(1-\beta-\Omega_{m}\right)\right]^{2 / 3(1-\beta)}$. Portanto, desde o momento de transição entre os domínios da matéria e do vácuo até a expansão final do universo, a entropia é uma função côncava. Essa condição é necessária para que o universo tenda assintoticamente a um estágio de equilíbrio.

Dessa forma, a entropia total do universo está limitada ao valor de:

$$
S_{\text {max }} \equiv \lim _{a \rightarrow \infty} S=\frac{\pi k_{B} c^{2}}{l_{p l}^{2} H_{0}^{2}} \frac{(1-\beta)^{2}}{\left(1-\beta-\Omega_{m}\right)^{2}} \approx 10^{122} k_{B},
$$

considerando os valores de melhor ajuste para os parâmetros livres. Isso significa que o universo evolui assintoticamente ao equilíbrio térmico. 
Em suma, mostramos que a entropia total do modelo de decaimento do vácuo investigado sempre cresce com o tempo, seguindo dessa maneira inclusive nos estágios futuros do Universo. Mostramos também que a segunda derivada da entropia é negativa, ao menos nos estágios derradeiros da expansão cósmica. Do ponto de vista termodinâmico, a expansão acelerada pode ser entendida como um processo fora do equilíbrio evoluindo para um estado de equilíbrio termodinâmico em $a \rightarrow \infty$.

\subsection{Análise Observacional}

Nesta seção analisaremos os vínculos obtidos sobre o parâmetro de densidade da matéria $\Omega_{m}$ e sobre o parâmetro $\beta$, ligado à taxa de decaimento do termo de energia escura, conforme a expressão (8.3). Utilizamos um teste estatístico de $\chi^{2}$ considerando os 580 dados de supernovas do tipo Ia provenientes da compilação Union 2.1 e da razão CMB/BAO (Sollerman et al., 2009; Percival et al., 2010; Komatsu et al., 2010; Blake et al., 2011; Suzuki et al., 2012).

\subsubsection{Vínculos de Supernovas Tipo Ia}

Neste teste utilizamos a compilação Union 2.1, com dados referentes a 580 supernovas do tipo Ia. O conjunto de parâmetros $s=\left(\Omega_{m}, \beta\right)$ que fornece o melhor ajuste ao modelo foi encontrado utilizando uma estatística de $\chi^{2}$ :

$$
\chi^{2}=\sum_{i=1}^{580} \frac{\left[\mu_{p}^{i}(z \mid s)\left(z_{i}\right)-\mu_{o}^{i}(z \mid s)\right]^{2}}{\sigma_{i}^{2}},
$$

onde $\mu_{p}^{i}(z \mid s)=5 \log D_{L}+25$ é o valor calculado do módulo de distância para uma SN Ia com redshift z, $D_{L}$ é a distância de luminosidade em unidades de Megaparsec, $\mu_{o}^{i}(z \mid s)$ é o módulo de distância corrigido para a dada SN Ia em $z_{i}$ e $\sigma_{i}$ é a incerteza individual do módulo de distância.

\subsubsection{Vínculos da razão $C M B / B A O$}

Complementamos o teste com dados de SN Ia utilizando dados provenientes da combinação entre medidas das oscilações acústicas de bárions (BAO -Baryon Acoustic Oscilations) e medidas de radiação cósmica de fundo (CMB) (Sollerman et al., 2009). 
Vínculos menos dependentes de modelos podem ser obtidos do produto da escala acústica da $\mathrm{CMB}, l_{A}=\pi d_{A}\left(z_{*}\right) / r_{s}\left(z_{*}\right)$, e da razão $r_{s}\left(z_{d}\right) / D_{V}\left(z_{\mathrm{BAO}}\right)$ entre a escala do horizonte do som quando ocorre o desacoplamento dos bárions (a chamada drag epoch) e a escala de dilatação de BAO. Na expressão anterior, $d_{A}\left(z_{*}\right)$ é a distância de diâmetro angular comóvel na recombinação $\left(z_{*}=1091.3\right)$ e $r_{s}\left(z_{*}\right)$ é o horizonte comóvel do som no desacoplamento dos fótons (Komatsu et al., 2010). O redshift $z_{d} \sim 1020$ é o redshift da drag epoch, quando há o "congelamento" das oscilações acústicas, e a escala de dilatação é dada por $D_{V}(z)=\left[z r^{2}(z) / H(z)\right]^{1 / 3}$.

As medidas disponíveis da razão $r_{s}\left(z_{d}\right) / D_{V}(z)$ foram obtidas por Percival et al. (2010) (Sloan Digital Sky Survey-II), para os redshifts $z=0.20$ e $z=0.35$ :

$$
\begin{aligned}
& r_{s}\left(z_{d}\right) / D_{V}(z=0.20)=0.1905 \pm 0.0061 \\
& r_{s}\left(z_{d}\right) / D_{V}(z=0.35)=0.1097 \pm 0.0036
\end{aligned}
$$

e por Blake et al. (2011) (Wiggle $z$ ), para $z=0.6$ :

$$
r_{s}\left(z_{d}\right) / D_{V}(z=0.60)=0.0692 \pm 0.0033
$$

Da combinacão das medidas acima com a razão $r_{\mathrm{c}}\left(z_{*}\right) / r_{\mathrm{c}}\left(z_{d}\right)=1.044 \pm 0.019$ (Komatsu et al., 2008), obtemos

$$
\begin{aligned}
& f_{0.20}=d_{A}\left(z_{*}\right) / D_{V}(0.20)=17.55 \pm 0.65 \\
& f_{0.35}=d_{A}\left(z_{*}\right) / D_{V}(0.35)=10.10 \pm 0.38 \\
& f_{0.60}=d_{A}\left(z_{*}\right) / D_{V}(0.60)=6.65 \pm 0.32
\end{aligned}
$$

\subsubsection{Análise Conjunta}

Discutiremos agora os vínculos provenientes da combinação dos dois conjuntos de dados. Em nossa análise estatística, minimizamos a função $\chi_{T}^{2}=\chi_{S N}^{2}+\chi_{C M B / B A O}^{2}$, onde $\chi_{S N}^{2} \mathrm{e}$ $\chi_{C M B / B A O}^{2}$ são as funções $\chi^{2}$ para SN Ia e CMB/BAO, respectivamente.

Na figura 8.5.2, são mostrados os vínculos obtidos no espaço de parâmetros $\Omega_{m}-\beta$. As regiões azuladas mostram os limites de confiança obtidos pela análise de dados de SN Ia enquanto as regiões em laranja representam os limites obtidos da análise da razão CMB/BAO. As regiões tracejadas representam os limites obtidos da análise conjunta. 


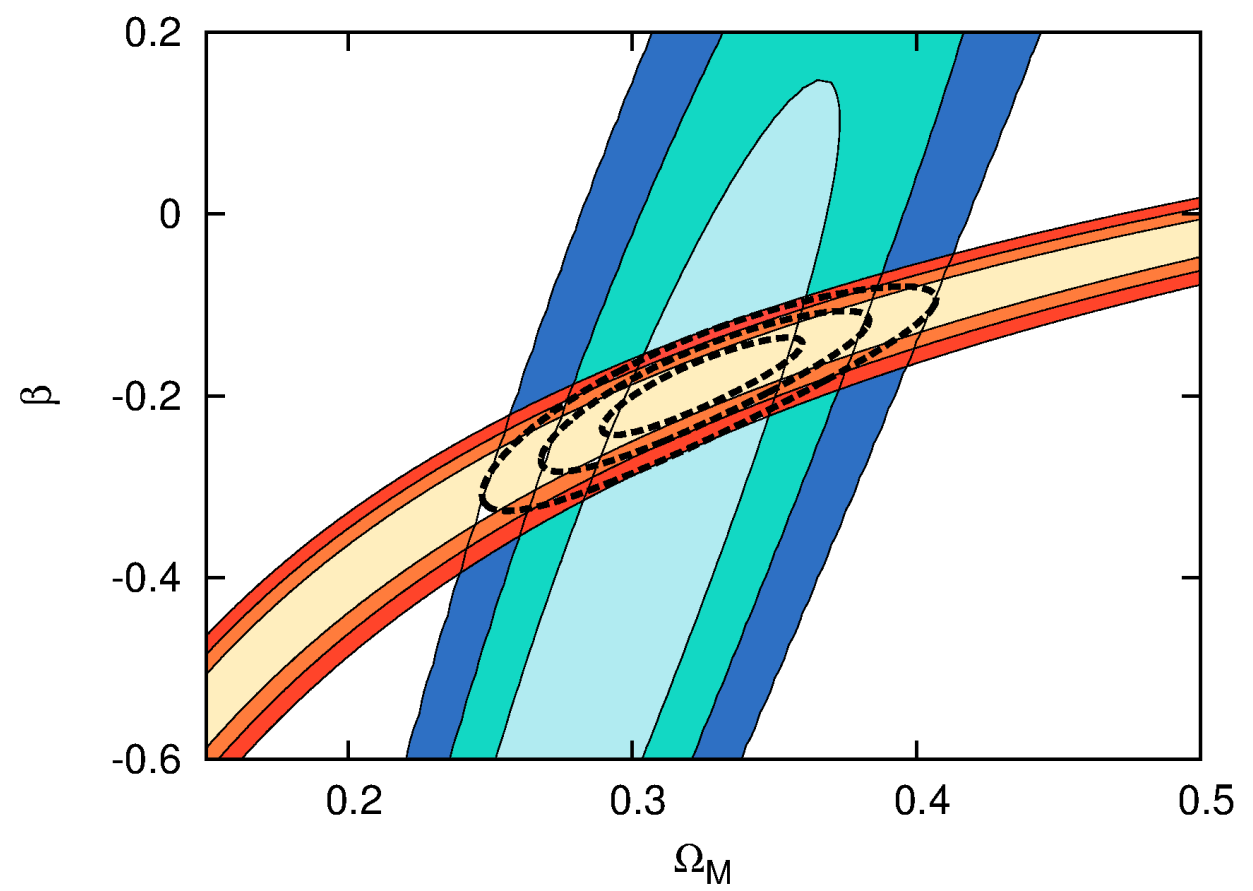

Figura 8.1: Resultados da análise estatística. Os vínculos de SNe Ia e da razão CMB/BAO são mostrados nos regiões em azul e laranja, respectivamente. As linhas tracejadas delimitam os vínculos obtidos da combinação dos dados observacionais para $68.3 \%$, 95.4\% e $99.73 \%$ de nível de confiança.

Através da marginalização sobre o parâmetro $h\left(H_{0}=100 h \mathrm{~km} \mathrm{~s}^{-1} \mathrm{Mpc}^{-1}\right)$, os vínculos obtidos da análise conjunta foram $\beta=-0.185_{-0.055+0.075+0.095}^{+0.045+} \Omega_{m}=0.325_{-0.035+0.060+0.080}^{+0.030}$ em $68.3 \%, 95.4 \%$ e $99.7 \%$ de nível de confiança, respectivamente. O valor de $\chi^{2}$ mínimo encontrado foi $\chi_{\min }^{2}=564$ para $\nu=581$ graus de liberdade. $\mathrm{O}$ valor reduzido de $\chi_{\nu}^{2}=0.97$, demonstrando que o modelo fornece um ajuste muito bom aos dados. Note que neste modelo de com criação de matéria por decaimento de vácuo, os limites sobre o parâmetro $\Omega_{m}$ são ligeiramente mais altos do que os valores de melhor ajustes do modelo $\Lambda$ CDM (Suzuki et al., 2012; Planck Collaboration XVI: Ade, P. A. R. et al., 2013).

Os parâmetros derivados do modelo podem ser estimados com base nos limites obtidos sobre $\Omega_{m}$ e $\beta$. Os valores derivados da análise conjunta são $q_{0}=-0.513_{-0.053}^{+0.053}, z_{t}=0.99_{-0.20}^{+0.19}$ e $t_{0} H_{0}=1.003_{-0.051}^{+0.049}$ (Ver tabela 8.1).

Os resultados obtidos em nossa análise são bastante semelhantes a outros resultados na literatura. Basilakos (2009), por exemplo, obteve limites sobre os parâmetros $\Omega_{m}$ e $\beta$ plenamente compatíveis com os apresentados aqui, utilizando a amostra com 307 SN Ia do catálogo Union (Kowalski et al., 2008). Além disso, nessa análise foram utilizados apenas o dado em $z_{B A O}=0.35$ para o parâmetro $\mathcal{A}$ derivado das observações de BAO e de forma 
não combinada com o dado de shift parameter $\mathcal{R}$ (Eisenstein et al., 2005).

Conforme discutido em Doran et al. (2007), esses observáveis foram obtidos no contexto do modelo $\Lambda$ CDM e podem ser considerados uma boa aproximação apenas para algumas classes de modelos de energia escura. Por exemplo, na estimativa do parâmetro de BAO é implicitamente assumido que a evolução das perturbações de densidades na era da matéria seja inalterada em relação ao modelo $\Lambda$ CDM e também que a distância comóvel do horizonte na época da equipartição entre matéria e radiação varie com $\left(\Omega_{m} H_{0}^{2}\right)^{-1}$. Entretanto, a produção de matéria em modelos com decaimento do vácuo pode modificar a dependência com $\Omega_{m}$ da distância do horizonte na equipartição e, portanto, a análise com o uso direto dessas grandezas deve ser evitada em modelos alternativos. Em nossa análise, contornamos essa questão utilizando a combinação das grandezas $r_{s}\left(z_{d}\right)$ e $D_{V}$, cuja razão é fracamente dependente de modelos (Sollerman et al., 2009).

Assumindo um acoplamento entre o fluido relativístico e o cosmológico $\left(\beta_{r} \neq 0\right)$. Birkel e Sarkar (1997) mostraram que a nucleossíntese primordial limita o termo de acoplamento em $\beta_{\underline{r}}<0.13$. O mesmo tipo de acoplamento fora discutido posteriormente por Lima et al. (2000), onde o limite obtido no termo de acoplamento foi $\beta_{r}<0.16$. Vínculos mais restritivos sobre o parâmetro $\beta_{r}$ foram obtidos através do efeito Sunyaev-Zeldovich. Por exemplo, em Luzzi et al. (2009), foi mostrado que o acoplamento leva a uma modificação na lei de temperatura usual da CMB, obtendo $\beta_{r}=0.041_{-0.041}^{+0.038}$. Entretanto, devemos ressaltar que tais resultados não podem ser levados em conta em nosso modelo, haja vista que estamos considerando apenas o acoplamento entre o termos de energia e matéria escura, assim como as épocas de domínio gravitacional desses termos.

\begin{tabular}{|crc|}
\hline \hline Parâmetro & best-fit & Incerteza ${ }_{-1 \sigma}^{+1 \sigma}$ \\
\hline \hline$\Omega_{m}$ & 0.325 & ${ }_{-0.035}^{+0.035}$ \\
$\beta$ & -0.185 & ${ }_{-0.055}^{+0.045}$ \\
$\bar{\sigma} / H_{0}$ & 2.58 & ${ }_{-0.27}^{+0.24}$ \\
$q_{0}$ & -0.513 & ${ }_{-0.053}^{+0.053}$ \\
$z_{t}$ & 0.99 & ${ }_{-0.20}^{+0.19}$ \\
$t_{0} H_{0}$ & 1.003 & ${ }_{-0.051}^{+0.049}$ \\
\hline
\end{tabular}

Tabela 8.1 - Resultados da análise estatística combinada. 


\subsection{Descrição de Campo Escalar}

Embora os modelos com $\Lambda(t)$ sejam uma abordagem interessante para tratar o problema da constante cosmológica, a forma da evolução temporal de termo de energia escura ainda precisa ser definida fenomenologicamente, haja vista a dificuldade em se estabelecer tais comportamentos partindo de primeiros princípios.

Uma possível maneira de buscar a motivação física para tais modelos é através de sua descrição na linguagem da teoria de campos, sendo o modo mais simples por meio de campos escalares. Desenvolvemos aqui uma derivação do modelo com $\Lambda(\mathrm{t})$ variável a partir de uma física mais fundamental com base na proposta de Maia e Lima (2002). Seguindo essa abordagem, definimos a função

$$
\gamma_{*} \equiv-\frac{2 \dot{H}}{3 H^{2}}=1-\frac{\Lambda}{3 H^{2}}
$$

sendo o modelo $\Lambda(t)$ definido através do parâmetro dependente do tempo $\gamma_{\star}$.

Especificamente, no modelo estudado aqui, a função $\gamma_{\star}$ assume a forma:

$$
\gamma_{*}=\frac{3(1-\beta) H^{2}-\bar{\sigma} H}{3 H^{2}}
$$

Substituindo a densidade de energia e a pressão do vácuo nas equações (8.1) e (8.2) pelas expressões de correspondentes em campos escalares, obtemos:

$$
\Lambda / 8 \pi G \rightarrow \rho_{\phi}=\dot{\phi}^{2} / 2+V(\phi)
$$

e

$$
-\Lambda / 8 \pi G \rightarrow p_{\phi}=\dot{\phi}^{2} / 2-V(\phi)
$$

onde $\rho_{\phi}$ e $p_{\phi}$ são, respectivamente, a densidade de energia e a pressão associadas ao campo escalar $\phi$, cujo potencial é $V(\phi)$.

Podemos separar as contribuições do campo escalar através das expressões (8.1), (8.2), (8.49) e (8.50). definindo o parâmetro $x \equiv \dot{\phi}^{2} /\left(\dot{\phi}^{2}+\rho_{d m}\right)$ com $0<x<1$. ou seia. (Maia e Lima, 2002):

$$
\dot{\phi}^{2}=\frac{3 H^{2}}{8 \pi G} \gamma_{*} x
$$

$\mathrm{e}$

$$
V(\phi)=\frac{3 H^{2}}{8 \pi G}\left[1-\gamma_{*}\left(1-\frac{x}{2}\right)\right]
$$




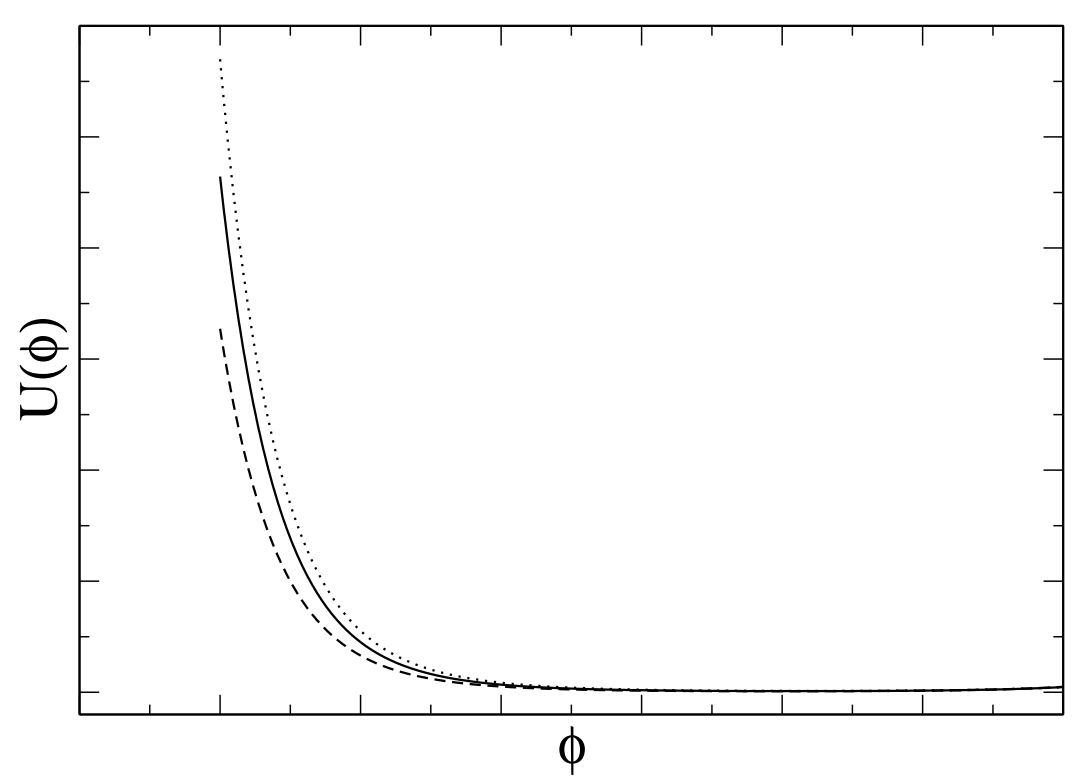

Figura 8.2: Evolução do potencial do campo escalar em unidades da densidade de energia crítica hoje $U(\phi) \equiv V(\phi) / \rho_{c, 0}$. As curvas pontilhada, sólida e tracejada correspondem respectivamente aos valores do limite inferior, melhor ajuste e limite superior dos parâmetros $\Omega_{m}$ e $\beta$ em $1 \sigma$ de nível de confiança Andrade-Oliveira, F. et al., 2014).

As equações (8.1) e (8.2) ligam diretamente o campo e seu potencial às grandezas relacionadas ao termo de energia escura $\Lambda(\mathrm{t})$. Da equação (8.51), é possível mostrar que o campo $\phi$ em função do parâmetro de Hubble é dado por:

$$
\phi-\phi_{0}= \pm \frac{1}{\sqrt{6 \pi G}} \int_{H}^{H_{0}} \sqrt{\frac{x}{\gamma_{*}}} \frac{d H}{H} .
$$

Com auxilio das equações (8.48) e (8.53), integramos a expressão (8.53) e obtemos:

$$
\phi-\phi_{0}=-k^{-1} \ln \left[\frac{\sqrt{H-A}+\sqrt{H}}{\sqrt{H_{0}-A}+\sqrt{H_{0}}}\right] \text {, }
$$

onde $k=\sqrt{3 \pi G(1-\beta) / 2 x}, A=\bar{\sigma} / 3(1-\beta)$ e consideramos o sinal positivo na equação (8.53). Utilizando a equação (8.52), obtemos

$$
V(\phi)=\frac{D^{2}}{64 \pi G}[(2-x) \bar{\sigma}+B f(\phi)] f(\phi)
$$

em que

$$
f(\phi)=\left[e^{-2 k\left(\phi-\phi_{0}\right)}+\left(\frac{A}{D^{2}}\right)^{2} e^{2 k\left(\phi-\phi_{0}\right)}+2 \frac{A}{D^{2}}\right]
$$


$\operatorname{com} B=3 D^{2}[2 \beta+(1-\beta) x] / 4$ e $D=\sqrt{H_{0}-A}+\sqrt{H_{0}}$. Ao assumir $\bar{\sigma}=0 \Longrightarrow A=0$, o potencial toma a forma de uma função exponencial simples sendo reduzido ao potencial originalmente encontrado por Maia e Lima (2002).

É interessante notar que potenciais com duas funções exponenciais, como o mostrado na equação (8.55), foram considerados na literatura como exemplos viáveis de modelos de quintessência - como, por exemplo, em Sen e Sethi (2002). Em Järv et al. (2004), um potencial também formado pela soma de dois termos exponenciais emerge através da redução dimensional em teorias-M, tendo consequências para a fase de aceleração tardia do universo.

Na figura (8.6), é mostrado o comportamento do potencial do campo escalar em unidades da densidade de energia crítica hoje $\left[U(\phi) \equiv V(\phi) / \rho_{c, 0}\right]$ para alguns valores selecionados dos parâmetros livres do modelo, a saber, $\Omega_{m}=(0.29,0.325,0.36)$ e $\beta=$ $(-0.24,-0.185,-0.14)$. Tais valores correspondem ao limite inferior, de melhor ajuste e limite superior dos parâmetros $\Omega_{m}$ e $\beta$ em $1 \sigma$ de nível de confiança.

Consideramos também o caso $x=0.2$. Este parâmetro codifica a quantidade de energia do potencial $V(\phi)$ que é transferida a cada um dos componentes, durante a evolução do universo. É possível notar que as curvas apresentadas possuem comportamentos parecidos.

Embora a descrição de campo escalar acoplado apresente também uma arbitrariedade no tipo de interação entre campo escalar e matéria escura, a descrição na linguagem da teoria de campos possui aspectos interessantes. Modelos de campo escalar acoplado podem contornar o problema da constante cosmológica, utilizando os dados observacionais para estabelecer a natureza da interação entre as componentes de campo escalar e de matéria escura e, portanto, a forma do potencial responsável pela fase acelerada do Universo. Além disso, ao implementarmos uma descrição do modelo por um campo escalar acoplado, a lagrangiana associada pode ser usada para estender a validade do modelo para outros espaços-tempos e para outras teorias gravitacionais.

\subsection{Discussões}

Neste capítulo, investigamos um cenário cosmológico com o decaimento do vácuo, cujo termo de energia escura é fenomenologicamente descrito em função de uma série de potências do parâmetro de Hubble em até segunda ordem. Discutimos alguns aspectos 
termodinâmicos do modelo, de acordo com a segunda lei generalizada da termodinâmica. Desse ponto de vista, mostramos que o modelo descreve a evolução do universo como um processo termodinâmico fora do equilíbrio, mas assintoticamente tendendo ao equilíbrio termodinâmico.

Vinculamos os parâmetros livres do modelo através de uma análise estatística envolvendo medidas observacionais de 580 SNe Ia (Suzuki et al., 2012) e da razão entre os picos das Oscilaç̃es Acústicas de Bárions e o shift parameter da Radiação Cósmica de Fundo (Sollerman et al., 2009; Komatsu et al., 2010; Percival et al., 2010; Blake et al., 2011). Os limites obtidos em $68 \%$ de nível de confiança foram $\Omega_{m}=0.325_{-0.035}^{+0.035}$ e $\beta=-0.185_{-0.055}^{+0.045}$ com $\chi^{2}$ reduzido, $\chi_{\nu}^{2}=0.97$, mostrando que o modelo fornece um ótimo ajuste aos dados observacionais.

Utilizando os valores de melhor ajuste dos parâmetro livres, pudemos estimar os valores atuais para algumas grandezas que caracterizam a dinâmica do modelo. O parâmetro de desaceleração hoje é $q_{0}=-0.513_{-0.053}^{+0.053}$, o redshift de transição é $z_{t}=0.99_{-0.20}^{+0.19}$ e a idade do universo hoje $t_{0} H_{0}=1.003_{-0.051}^{+0.049}$. Esses valores são compatíveis com as previsões obtidas no modelo padrão.

Deduzimos ainda uma descrição do modelo em função de um campo escalar, seguindo o método desenvolvido em Maia e Lima (2002). Nessa descrição, pudemos identificar o presente modelo $\Lambda(\mathrm{t}) \mathrm{CDM}$ a um campo quintessencial acoplado com o potencial (8.55). Como o cenário $\Lambda(\mathrm{t}) \mathrm{CDM}$ descrito é bastante geral, tendo inclusive como caso particular diversas outras propostas já descritas na literatura, a descrição em campo quintessencial acoplado é dinamicamente equivalente a muitos modelos de decaimento do vácuo previamente propostos. 
Capítulo 9

\section{Conclusões}

O acúmulo de dados observacionais nas últimas décadas, culminando na descoberta de que o universo passa por uma fase de expansão acelerada, abriu uma nova era na cosmologia. No contexto da Teoria da Relatividade Geral (TRG), a forma mais simples de acelerar o universo é supor a existência de uma componente escura extra em adição à matéria escura e que tenha como propriedade a pressão negativa, a chamada energia escura. Sendo o universo composto de $70 \%$ de energia escura segundo o modelo padrão, a compreensão da natureza dessa componente e da origem da aceleração cósmica está certamente entre as questões mais relevantes e desafiadoras na Ciência hoje, estimulando a elaboração de diversas propostas na literatura.

Iniciamos esta tese revisando alguns conceitos básicos da Teoria da Relatividade Geral e do modelo padrão da cosmologia (capítulo 2). Vimos que a imposição do princípio cosmológico leva à métrica de FLRW e então discutimos a dinâmica cósmica e as principais observações que dão suporte ao modelo padrão.

No capítulo 3, vimos que, embora o modelo $\Lambda$ CDM tenha ótima capacidade de ajustar as observações cosmológicas, a natureza da constante cosmológica/energia do vácuo ainda não é compreendida, possuindo graves problemas conceituais. Discutimos alguns dos diversos modelos de energia escura que buscam uma explicação alternativa para a aceleração cósmica.

No capítulo 4 discutimos a criacão de partículas no contexto cosmológico (Prigogine

et al., 1989; Lima et al., 1991; Calvão et al., 1992). Mostramos que nos modelos com criação de matéria escura fria (CCDM), o processo irreversível de criação de matéria à custa do campo gravitacional gera um termo de pressão negativa em nível das equações de Einstein. Dessa forma, essa classe de modelos sem constante cosmológica pode ser considerada como 
alternativa à energia escura.

O capítulo [5] se iniciou pela discussão da dinâmica cosmológica do modelo LJO em background, conforme publicamos em Lima et al. (2010). Caracterizado pela taxa de criação $\Gamma_{L J O}=3 \alpha H \frac{\rho_{c 0}}{\rho_{m}}$, o modelo apresentado tem a notável propriedade de recuperar a mesma história cósmica do modelo $\Lambda$ CDM. Discutimos a dinâmica do modelo com criação de matéria escura fria e do caso com criação de matéria escura com pressão $p_{m}=\varepsilon \rho_{m}$. Os limites obtidos com $95.4 \%$ de significância estatística para o caso de criação de matéria escura fria foram $\Omega_{m}=1.004_{-0.446}^{+0.390}$ e $\alpha=0.725_{-0.287}^{+0.240}$, no modelo com curvatura, e $\alpha=$ $0.722_{-0.039}^{+0.039}$, no caso plano. No caso com criação de matéria escura com pressão, os dados de SNe Ia não foram capazes de gerar limites restritivos, onde obtivemos $\alpha=0.723_{-0.319}^{+0.111} \mathrm{e}$ $\varepsilon=0.001_{-0.422}^{+0.333}$, com $68.3 \%$ de confiança estatística.

No capítulo 6, investigamos as perturbações lineares de densidade no modelo LJO, conforme apresentamos em Lima et al. (2011) e Jesus et al. (2011). Discutimos inicialmente o formalismo Neo-Newtoniano, proposto por Lima et al. (1997) e sua validade em comparação ao formalismo relativístico não perturbado. Em seguida obtivemos as equações de perturbações lineares de densidade para um modelo CCDM genérico e com velocidade efetiva do som arbitrária. Comparamos as previsões do modelo LJO e do modelo $\Lambda$ CDM, aplicando o formalismo desenvolvido ao modelo LJO plano. No formalismo utilizado, as medidas observacionais indicam que o modelo tem excelente capacidade de descrever as observações para o caso $c_{e f f}^{2}=-1$. Porém, nesse caso o modelo pode apresentar problemas nas perturbações. Essa questão pode ser contornada através da decomposição da matéria escura em duas componentes, a saber, uma componente que se aglomera e outra com comportamento semelhante à energia escura, conforme a abordagem relativística de Ramos et al. (2014). Assumindo essa hipótese, os modelos $\Lambda$ CDM e LJO passam a ser completamente degenerados, do ponto de vista cosmológico.

No capítulo 7, propusemos um novo modelo CCDM caracterizado pela taxa de criação de matéria $\Gamma=3 \alpha \frac{H_{0}^{2}}{H}$ (Jesus e Andrade-Oliveira, F., 2015). Mostramos que o modelo proposto é capaz de ajustar os dados de SNe Ia e $H(z)$ com qualidade similar à dos modelos $\Lambda$ CDM e LJO. Entretanto, embora os três modelos possuam taxas de expansão semelhantes, o problema da degenerescência intrínseca é naturalmente ausente na presença da matéria bariônica e/ou curvatura. Os vínculos obtidos sobre os parâmetros livres para o caso com curvatura arbitrária foram $\alpha=0.792_{-0.093}^{+0.260}, \Omega_{k}=0.05_{-0.40}^{+0.42}$, com $95.4 \%$ de 
confiança estatística. Adicionando um prior sobre a curvatura, os vínculos obtidos foram $\alpha=0.768_{-0.051}^{+0.058}$ e $\Omega_{k}=-0.036_{-0.110}^{+0.110}$.

No capítulo 8, discutimos a viabilidade um modelo de decaimento do vácuo e a sua descrição por campo escalar, de acordo com o trabalho publicado em Andrade-Oliveira, F. et al. (2014). Consideramos o termo de vácuo $\Lambda(\mathrm{t})$ como sendo uma série truncada do parâmetro de Hubble $H$ e derivamos as equações dinâmicas do modelo. Utilizando a segunda lei da termodinâmica generalizada (Mimoso e Pavón, 2013), discutimos a termodinâmica da expansão cosmológica desse modelo $\Lambda(t)$ CDM. Vinculamos os parâmetros livres através das observações de SNe Ia e da razão CMB/BAO, obtendo $\beta=-0.185_{-0.085}^{+0.075}$ e $\Omega_{m}=0.325_{-0.060}^{+0.060}$ para $95.4 \%$ de confiança estatística. Finalmente, derivamos o modelo fenomenológico em termos de um campo escalar decaindo em um fluido de matéria escura, conforme a proposta de Maia e Lima (2002). Uma vez que o modelo de decaimento tem como casos particulares diversas outras propostas na literatura, a descrição em campo escalar pode ser estendida a estes outros. Além disso, através da descrição pela Lagrangiana associada, o modelo pode ser considerado em teorias gravitacionais alternativas ou mesmo em regimes de energia além dos limites de validade da TRG.

Em suma, os candidatos apresentados aqui possuem capacidades semelhantes de descrever as observações que o modelo de concordância cósmica $(\Lambda \mathrm{CDM})$. Na verdade, as atuais discussões na literatura apontam que os modelos LJO e $\Lambda$ CDM possuem uma equivalência completa do ponto de vista cosmológico sendo, portanto, o modelo apresentado um candidato observacionalmente viável. O mesmo pode ser dito do cenário de Jesus \& Oliveira, onde a degenerescência entre modelos é naturalmente contornada pela existência da componente bariônica e o modelo se mostra capaz de ajustar as observações com o mesmo número de parâmetros livres que os modelos LJO e $\Lambda$ CDM. Naturalmente, tais modelos não são apenas uma reinterpretação da cosmologia $\Lambda$ CDM, mas também uma possível explicação à aceleração cósmica sem a necessidade de um fluído exótico e, portanto, livre dos problemas da constante cosmológica e da coincidência cósmica.

Contudo, deve-se ressaltar que em ambos os modelos CCDM a taxa de criação $\Gamma$ não é determinada de primeiros princípios. Também não é claro qual o melhor método para tratarem-se as perturbações em modelos CCDM, assim como não é clara de qual maneira em que a aglomeração perturba a taxa de criação, i.e., qual a forma funcional da velocidade efetiva do som nesse modelo. Além disso, desconsiderar a separação das componentes 
de matéria escura no modelo LJO gera uma séria tensão entre os vínculos obtidos em background e os provenientes da análise das perturbações de densidade.

Estudos futuros deverão focar na obtenção do espectro de potências e no uso de técnicas de Monte Carlo, como por exemplo o COSMOMC Lewis e Bridle (2002), para a restrição dos parâmetros livres dos modelos utilizando medidas da CMB. Esperamos com isso ganhar um novo insight sobre o processo de criação no universo inomogêneo.

Tentativas da determinação da massa e da taxa de criação dos modelos CCDM provenientes das previsões em teoria quântica de campos em espaços curvos podem trazer vínculos independentes (Jesus e Pereira, 2014). Embora as estimativas utilizando dados cosmológicos ainda sejam pouco restritivas, claramente a detecção experimental de partículas de matéria escura é extremamente menos complicada que a detecção da energia do vácuo por vias não cosmológicas, como por exemplo, através de efeito sobre as órbitas no sistema solar ou medidas do Lamb shift (Calloni et al., 2002; Sorge, 2005; Polchinski, 2006; Martin, 2012).

Em relação aos modelos $\Lambda(\mathrm{t}) \mathrm{CDM}$, admitindo-se que a energia do vácuo realmente possua um papel gravitacional, é extremamente relevante discutirmos a possibilidade de interação entre as componentes do setor escuro, responsável por 95\% da energia do universo. Modelos com decaimento do vácuo têm se saído muito bem nesse papel, sendo capazes de explicar a aceleração e contornando o problema da constante cosmológica. Nesse caso, algumas análises envolvendo medidas de estruturas têm dado fortes indícios da existência de uma interação no setor escuro Salvatelli et al. (2014). Deve-se ressaltar, entretanto, que a evolução temporal de $\Lambda$ nesses modelos ainda é determinada fenomenologicamente mesmo que em alguns casos sejam inspiradas por resultados da teoria quântica de campos (Shapiro e Solà, 2009; Lima et al., 2013; Perico et al., 2013).

De modo geral, a grande quantidade de dados previstos pelos projetos futuros e o maior controle sobre os erros sistemáticos das observações vão beneficiar enormemente os estudos em cosmologia.

Finalmente, esperamos continuar participando deste processo não apenas através de análises estatísticas mais refinadas, mas também através da formulação de alternativas teóricas que contribuam para as discussões acerca da natureza da energia escura, aceleração cósmica e composição do universo.

As observações cosmológicas das últimas décadas trouxeram um avanço inegável à 
cosmologia, estabelecendo o modelo $\Lambda$ CDM como modelo padrão. Embora o modelo tenha grande êxito em descrever as observações cosmológicas, admitir que esse modelo encerre toda a física necessária para a próxima geração de testes cosmológicos é ignorar o fato de que, além de não compreendermos a sua física subjacente, existe todo um espectro de propostas alternativas muito interessantes a ser explorado. 


\section{Referências Bibliográficas}

Abdel-Rahman A., Singularity-free decaying-vacuum cosmologies, Phys. Rev. D, 1992, vol. 45 , p. 3497

Abramo L. R., Batista R. C., Liberato L., Rosenfeld R., Structure formation in the presence of dark energy perturbations, J. Cosmology Astropart. Phys., 2007, vol. 11, p. 12

Aguirre A. N., Dust versus Cosmic Acceleration, ApJ, 1999, vol. 512, p. L19

Alcaniz J. S., Jain D., Dev A., High-redshift objects and the generalized Chaplygin gas, Phys. Rev. D, 2003, vol. 67, p. 043514

Alcaniz J. S., Lima J. A. S., New Limits on $\Omega_{\Lambda}$ and $\Omega_{M}$ from Old Galaxies at High Redshift, ApJ, 1999, vol. 521, p. L87

Alcaniz J. S., Lima J. A. S., Dark Energy and the Epoch of Galaxy Formation, ApJ, 2001, vol. 550 , p. L133

Alcaniz J. S., Lima J. A. S., Interpreting cosmological vacuum decay, Phys. Rev. D, 2005a, vol. 72 , p. 063516

Alcaniz J. S., Lima J. A. S., Measuring the Chaplygin Gas Equation of State from Angular and Luminosity Distances, ApJ, 2005b, vol. 618, p. 16

Allen S. W., Rapetti D. A., Schmidt R. W., Ebeling H., Morris R. G., Fabian A. C., Improved constraints on dark energy from Chandra X-ray observations of the largest relaxed galaxy clusters, MNRAS, 2008, vol. 383, p. 879 
Allen S. W., Schmidt R. W., Fabian A. C., Cosmological constraints from the X-ray gas mass fraction in relaxed lensing clusters observed with Chandra, MNRAS, 2002, vol. 334, p. L11

Alpher R. A., Bethe H., Gamow G., The Origin of Chemical Elements, Physical Review, 1948, vol. 73 , p. 803

Alpher R. A., Herman R., Evolution of the Universe, Nature, 1948, vol. 162, p. 774

Amendola L., Finelli F., Burigana C., Carturan D., WMAP and the Generalized Chaplygin Gas, JCAP, 2003, vol. 0307, p. 005

Amendola L., Tsujikawa S., Dark Energy : Theory and Observations by Luca Amendola and Shinji Tsujikawa. Cambridge University Press, 2010. ISBN: 9780521516006, 2010

Anderson G. W., Carroll S. M., Dark matter with time dependent mass, 1997

Andrade-Oliveira, F. Costa F. E. M., Lima J. A. S., Decaying vacuum cosmology and its scalar field description, Classical and Quantum Gravity, 2014, vol. 31, p. 045004

Arkani-Hamed N., Dimopoulos S., Dvali G., Phenomenology, astrophysics, and cosmology of theories with submillimeter dimensions and TeV scale quantum gravity, Phys. Rev. D, 1999, vol. 59, p. 086004

Aver E., Olive K. A., Porter R. L., Skillman E. D., The primordial helium abundance from updated emissivities, J. Cosmology Astropart. Phys., 2013, vol. 11, p. 17

Bak D., Rey S.-J., LETTER TO THE EDITOR: Cosmic holography ${ }^{+}$Cosmic holography, Classical and Quantum Gravity, 2000, vol. 17, p. L83

Basilakos S., Cosmological implications and structure formation from a time varying vacuum, MNRAS, 2009, vol. 395, p. 2347

Beesham A., Cosmological models with a variable cosmological term and bulk viscous models, Phys. Rev. D, 1993, vol. 48, p. 3539

Begeman K. G., Broeils A. H., Sanders R. H., Extended rotation curves of spiral galaxies - Dark haloes and modified dynamics, MNRAS, 1991, vol. 249, p. 523 
Bekenstein J., Black holes and the second law, Lett.Nuovo Cim., 1972, vol. 4, p. 737

Bekenstein J., Black Holes and Entropy, Phys. Rev. D, 1973, vol. 7, p. 2333

Bento M. C., Bertolami O., Sen A. A., Generalized Chaplygin gas, accelerated expansion, and dark-energy-matter unification, Phys. Rev. D, 2002, vol. 66, p. 043507

Betoule M., Kessler R., Guy J., et al. Improved cosmological constraints from a joint analysis of the SDSS-II and SNLS supernova samples, A\&A, 2014, vol. 568, p. A22

Bilic N., Tupper G. B., Viollier R. D., Unification of dark matter and dark energy: The inhomogeneous Chaplygin gas, Phys. Lett., 2002, vol. B535, p. 17

Birkel M., Sarkar S., Nucleosynthesis bounds on a time-varying cosmological _constant_, Astroparticle Physics, 1997, vol. 6, p. 197

Birrell N. D., Davies P. C. W., Quantum Quantum in Curved Space, 1982

Blake C., Brough S., Colless M., et al. The WiggleZ Dark Energy Survey: joint measurements of the expansion and growth history at $z<1$, MNRAS, 2012, vol. 425, p. 405

Blake C., Davis T., Poole G. B., et al. The WiggleZ Dark Energy Survey: testing the cosmological model with baryon acoustic oscillations at $\mathrm{z}=0.6$, MNRAS, 2011, vol. 415, p. 2892

Blumenthal G. R., Faber S. M., Primack J. R., Rees M. J., Formation of galaxies and large-scale structure with cold dark matter, Nature, 1984, vol. 311, p. 517

Borges H. A., Carneiro S., Fabris J. C., Evolution of density perturbations in decaying vacuum cosmology: The case of nonzero perturbations in the cosmological term, Phys. Rev. D, 2008, vol. 78, p. 123522

Borges H. A., Carneiro S., Fabris J. C., Zimdahl W., Non-adiabatic Chaplygin gas, Physics Letters B, 2013, vol. 727, p. 37

Bradač M., Allen S. W., Treu T., Ebeling H., Massey R., Morris R. G., von der Linden A., Applegate D., Revealing the Properties of Dark Matter in the Merging Cluster MACS J0025.4-1222, ApJ, 2008, vol. 687, p. 959 
Bronstein M. P., The problem of the possible theory of the World as a whole, Uspekhi Astronomicheskih Nauk, 1933, Coll(Issue).3, pp. 3-30 (Russian title: K voprosu o vozmojnoi teorii mira kak tselogo), 1933, vol. 3, p. 3

Burbidge E. M., Burbidge G. R., Fowler W. A., Hoyle F., Synthesis of the Elements in Stars, Reviews of Modern Physics, 1957, vol. 29, p. 547

Busca N. G., Delubac T., Rich J., et al. Baryon acoustic oscillations in the Ly $\alpha$ forest of BOSS quasars, A\&A, 2013, vol. 552, p. A96

Cai R.-G., Cao L.-M., Hu Y.-P., Hawking Radiation of Apparent Horizon in a FRW Universe, Class.Quant.Grav., 2009, vol. 26, p. 155018

Cai R.-G., Kim S. P., First Law of Thermodynamics and Friedmann Equations of Friedmann Robertson Walker Universe, Journal of High Energy Physics, 2005, vol. 2, p. 50

Caldwell R. R., An introduction to quintessence., Brazilian Journal of Physics, 2000, vol. 30 , p. 215

Caldwell R. R., Dave R., Steinhardt P. J., Cosmological Imprint of an Energy Component with General Equation of State, Physical Review Letters, 1998, vol. 80, p. 1582

Caldwell R. R., Kamionkowski M., Weinberg N. N., Phantom Energy: Dark Energy with $\omega<-1$ Causes a Cosmic Doomsday, Physical Review Letters, 2003, vol. 91, p. 071301

Caldwell, R. R. A phantom menace? Cosmological consequences of a dark energy component with super-negative equation of state, Physics Letters B, 2002, vol. 545, p. 23

Callen H. B., Thermodynamics and an Introduction to Thermostatistics, 2nd Edition. Wiley-VCH, 1985

Calloni E., Di Fiore L., Esposito G., Milano L., Rosa L., Vacuum fluctuation force on a rigid Casimir cavity in a gravitational field, Physics Letters A, 2002, vol. 297, p. 328

Calvão M. O., Waga I., Lima J. A. S., On the thermodynamics of matter creation in cosmology., Physics Letters A, 1992, vol. 162, p. 223 
Campos J. P., Fabris J. C., Perez R., Piattella O. F., Velten H., Does Chaplygin gas have salvation?, European Physical Journal C, 2013, vol. 73, p. 2357

Capozziello S., Carloni S., Troisi A., Quintessence without scalar fields, ArXiv Astrophysics e-prints, 2003

Caramês T. R. P., Fabris J. C., Velten H. E. S., Spherical collapse for unified dark matter models, Phys. Rev. D, 2014, vol. 89, p. 083533

Carneiro S., Pigozzo C., Borges H. A., Alcaniz J. S., Supernova constraints on decaying vacuum cosmology, Phys. Rev. D, 2006, vol. 74, p. 023532

Carroll S. M., Duvvuri V., Trodden M., Turner M. S., Is cosmic speed-up due to new gravitational physics?, Phys. Rev. D, 2004, vol. 70, p. 043528

Carroll S. M., Hoffman M., Trodden M., Can the dark energy equation-of-state parameter w be less than -1?, Phys. Rev. D, 2003, vol. 68, p. 023509

Carturan D., Finelli F., Cosmological effects of a class of fluid dark energy models, Phys. Rev. D, 2003, vol. 68, p. 103501

Carvalho F. C., Santos E. M., Alcaniz J. S., Santos J., Cosmological constraints from the Hubble parameter on $\mathrm{f}(\mathrm{R})$ cosmologies, J. Cosmology Astropart. Phys., 2008, vol. 9, p. 8

Carvalho J. C., Lima J. A. S., Waga I., Cosmological consequences of a time-dependent Lambda term, Phys. Rev. D, 1992, vol. 46, p. 2404

Casas J. A., Garcia-Bellido J., Quiros M., Scalar-tensor theories of gravity with Phi dependent masses, Classical and Quantum Gravity, 1992, vol. 9, p. 1371

Chen W., Wu Y., Implications of a cosmological constant varying as $R^{-2}$, Phys. Rev. D, 1990, vol. 41, p. 695

Chevallier M., Polarski D., Accelerating Universes with Scaling Dark Matter, International Journal of Modern Physics D, 2001, vol. 10, p. 213

Chiba T., Okabe T., Yamaguchi M., Kinetically driven quintessence, Phys. Rev. D, 2000, vol. 62 , p. 023511 
Chuang C.-H., Wang Y., Modelling the anisotropic two-point galaxy correlation function on small scales and single-probe measurements of $\mathrm{H}(\mathrm{z}), \mathrm{D}_{A}(\mathrm{z})$ and $\mathrm{f}(\mathrm{z}) \sigma_{8}(\mathrm{z})$ from the Sloan Digital Sky Survey DR7 luminous red galaxies, MNRAS, 2013, vol. 435, p. 255

Clarkson C., Establishing homogeneity of the universe in the shadow of dark energy, Comptes Rendus Physique, 2012, vol. 13, p. 682

Clarkson C., Bassett B., Lu T. H.-C., A General Test of the Copernican Principle, Physical Review Letters, 2008, vol. 101, p. 011301

Clifton T., Ferreira P. G., Padilla A., Skordis C., Modified gravity and cosmology, Phys. Rep., 2012, vol. 513, p. 1

Clowe D., Bradač M., Gonzalez A. H., Markevitch M., Randall S. W., Jones C., Zaritsky D., A Direct Empirical Proof of the Existence of Dark Matter, ApJ, 2006, vol. 648, p. L109

Cole S., Percival W. J., Peacock J. A., Norberg P., et al. The 2dF Galaxy Redshift Survey: power-spectrum analysis of the final data set and cosmological implications, MNRAS, 2005, vol. 362 , p. 505

Colless M., et al., The $2 \mathrm{dF}$ Galaxy Redshift Survey: Spectra and redshifts, Mon.Not.Roy.Astron.Soc., 2001, vol. 328, p. 1039

Copeland E. J., Sami M., Tsujikawa S., Dynamics of Dark Energy, International Journal of Modern Physics D, 2006, vol. 15, p. 1753

Costa F. E. M., Alcaniz J. S., Cosmological consequences of a possible $\Lambda$-dark matter interaction, Phys. Rev. D, 2010, vol. 81, p. 043506

Costa F. E. M., Alcaniz J. S., Maia J. M. F., Coupled quintessence and vacuum decay, Phys. Rev. D, 2008, vol. 77, p. 083516

Cunha J. V., Santos R. C., The Existence of AN Old Quasar at z=3.91 and its Implications for $\Lambda(\mathrm{t})$ Deflationary Cosmologies, International Journal of Modern Physics D, 2004, vol. 13 , p. 1321

Custodio P. S., Horvath J. E., Supermassive Black Holes May Be Limited by the Holographic Bound, General Relativity and Gravitation, 2003, vol. 35, p. 1337 
da Angela J., Shanks T., Croom S., Weilbacher P., Brunner R., et al., The 2dF-SDSS LRG and QSO Survey: QSO clustering and the L-z degeneracy, Mon.Not.Roy.Astron.Soc., 2008, vol. 383 , p. 565

Dadhich N., Universality, Gravity, the enigmatic Lambda and Beyond, ArXiv General Relativity and Quantum Cosmology e-prints, 2004

D'Agostini G., Asymmetric Uncertainties: Sources, Treatment and Potential Dangers, ArXiv Physics e-prints, 2004

Daly R. A., Guerra E. J., Quintessence, Cosmology, and Fanaroff-Riley Type IIb Radio Galaxies, AJ, 2002, vol. 124, p. 1831

de Sitter W., Einstein's theory of gravitation and its astronomical consequences. Third paper, MNRAS, 1917, vol. 78, p. 3

Del Popolo A., Pace F., Lima J., Spherical collapse model with shear and angular momentum in dark energy cosmologies, MNRAS, 2013, vol. 430, p. 628

Del Popolo A., Pace F., Maydanyuk S. P., Lima J. A. S., Jesus J. F., Shear and rotation in Chaplygin cosmology, Phys. Rev. D, 2013, vol. 87, p. 043527

Dev A., Alcaniz J. S., Jain D., Cosmological consequences of a Chaplygin gas dark energy, Phys. Rev. D, 2003, vol. 67, p. 023515

Dicke R. H., Peebles P. J. E., Roll P. G., Wilkinson D. T., Cosmic Black-Body Radiation., ApJ, 1965, vol. 142, p. 414

Doran M., Stern S., Thommes E., Baryon acoustic oscillations and dynamical dark energy, J. Cosmology Astropart. Phys., 2007, vol. 4, p. 15

Drell P. S., Loredo T. J., Wasserman I., Type IA Supernovae, Evolution, and the Cosmological Constant, ApJ, 2000, vol. 530, p. 593

Edwards D., Exact expressions for the properties of the zero-pressureFriedmann models, MNRAS, 1972, vol. 159, p. 51 
Einstein A., Kosmologische Betrachtungen zur allgemeinen Relativitätstheorie, Sitzungsberichte der Königlich Preußischen Akademie der Wissenschaften (Berlin), Seite 142-152., 1917, pp 142-152

Einstein A., de Sitter W., On the Relation between the Expansion and the Mean Density of the Universe, Proceedings of the National Academy of Science, 1932, vol. 18, p. 213

Eisenstein D. J., Zehavi I., Hogg D. W., et al. Detection of the Baryon Acoustic Peak in the Large-Scale Correlation Function of SDSS Luminous Red Galaxies, ApJ, 2005, vol. 633 , p. 560

Elizalde E., Nojiri S., Odintsov S. D., Wang P., Dark energy: Vacuum fluctuations, the effective phantom phase, and holography, Phys. Rev. D, 2005, vol. 71, p. 103504

Ellis G. F. R., Maartens R., MacCallum M. A. H., Relativistic Cosmology. Cambridge Univ. Press Cambridge UK, 2012

Evrard A. E., The intracluster gas fraction in X-ray clusters - Constraints on the clustered mass density, MNRAS, 1997, vol. 292, p. 289

Fabris J., Velten H., Neo-Newtonian cosmology: An intermediate step towards General Relativity, RBEF, 2012, vol. 4302

Fabris J. C., Goncalves S. V. B., de Souza P. E., Fitting the Supernova Type Ia Data with the Chaplygin Gas, ArXiv Astrophysics e-prints, 2002

Fabris J. C., Velten H. E. S., Zimdahl W., Matter power spectrum for the generalized Chaplygin gas model: The relativistic case, Phys. Rev. D, 2010, vol. 81, p. 087303

Farooq O., Ratra B., Hubble Parameter Measurement Constraints on the Cosmological Deceleration-Acceleration Transition Redshift, ApJ, 2013, vol. 766, p. L7

Fernandes R. A. A., de Carvalho J. P. M., Kamenshchik A. Y., Moschella U., da Silva A., Spherical "top-hat" collapse in general-Chaplygin-gas-dominated universes, Phys. Rev. D, 2012, vol. 85, p. 083501

Fixsen D. J., The Temperature of the Cosmic Microwave Background, ApJ, 2009, vol. 707, p. 916 
Fomin P. I., Gravitational instability of a vacuum and the cosmological problem, Akademiia Nauk Ukrains koi RSR Dopovidi Seriia Fiziko Matematichni ta Tekhnichni Nauki, 1975, pp 831-835

Forman W., Jones C., X-ray-imaging observations of clusters of galaxies, Ann. Rev. Astron. Astrophys., 1982, vol. 20, p. 547

França U., Rosenfeld R., Age constraints and fine tuning in variable-mass particle models, Phys. Rev. D, 2004, vol. 69, p. 063517

Freedman W. L., Madore B. F., Mould J. R., Hill R., Ferrarese L., Kennicutt R. C., Saha A., Stetson P. B., Graham J. A., Ford H., Hoessel J. G., Huchra J., Hughes S. M., Illingworth G. D., Distance to the Virgo cluster galaxy M100 from Hubble Space Telescope observations of Cepheids, Nature, 1994, vol. 371, p. 757

Freese K., Adams F. C., Frieman J. A., Mottola E., Cosmology with decaying vacuum energy, Nuclear Physics B, 1987, vol. 287, p. 797

Friedmann A., Über die Krümmung des Raumes, Zeitschrift für Physik, 1922, vol. 10, p. 377

Friedmann A., Über die Möglichkeit einer Welt mit konstanter negativer Krümmung des Raumes, Zeitschrift für Physik, 1924, vol. 21, p. 326

Frieman J. A., Turner M. S., Huterer D., Dark Energy and the Accelerating Universe, ARA\&A, 2008, vol. 46, p. 385

Fulling S. A., Parker L., Hu B. L., Particle Creation in Expanding Universes, Phys. Rev. D, 1974, vol. 10, p. 3905

Gamow G., Expanding Universe and the Origin of Elements, Physical Review, 1946, vol. 70, p. 572

Garriga J., Mukhanov V. F., Perturbations in k-inflation, Physics Letters B, 1999, vol. 458, p. 219

Gibbons G. W., Hawking S. W., Cosmological event horizons, thermodynamics, and particle creation, Phys. Rev. D, 1977, vol. 15, p. 2738 
Gilliland R. L., Nugent P. E., Phillips M. M., High-Redshift Supernovae in the Hubble Deep Field, ApJ, 1999, vol. 521, p. 30

Giodini S., Pierini D., Finoguenov A., et al. Stellar and Total Baryon Mass Fractions in Groups and Clusters Since Redshift 1, ApJ, 2009, vol. 703, p. 982

Gonzalez A. H., Zaritsky D., Zabludoff A. I., A Census of Baryons in Galaxy Clusters and Groups, ApJ, 2007, vol. 666, p. 147

Graef L., Costa F., Lima J., On the equivalence of $\Lambda(t)$ and gravitationally induced particle production cosmologies, Phys.Lett., 2014, vol. B728, p. 400

Grande J., Solà J., Stefancic H., XCDM: a cosmon model solution to the cosmological coincidence problem?, J. Cosmology Astropart. Phys., 2006, vol. 8, p. 11

Gregory P. C., Bayesian Logical Data Analysis for the Physical Sciences: A Comparative Approach with 'Mathematica' Support. Cambridge University Press Cambridge, UK, 2005

Grib A. A., Dorofeev V. Y., Creation of Particles and Entropy in the Early Friedmann Universe, International Journal of Modern Physics D, 1994, vol. 3, p. 731

Gurvits L. I., Kellermann K. I., Frey S., The "angular size - redshift" relation for compact radio structures in quasars and radio galaxies, A\&A, 1999, vol. 342, p. 378

Guzzo L., Pierleoni M., Meneux B., Branchini E., Fevre O. L., et al., A test of the nature of cosmic acceleration using galaxy redshift distortions, Nature, 2008, vol. 451, p. 541

Harrison E. R., Cosmology without general relativity, Annals of Physics, 1965, vol. 35, p. 437

Hawking S. W., Particle creation by black holes, Comm. Math. Phys., 1975, vol. 43, p. 199

Hawking S. W., The cosmological constant is probably zero, Physics Letters B, 1984, vol. 134, p. 403

Hawking S. W., The Cosmological Constant is Probably Zero, Advanced Series in Astrophysics and Cosmology, 1993, vol. 8, p. 276 
Heavens A., Statistical techniques in cosmology, ArXiv e-prints, 2009

Hinshaw G., Spergel D. N., Verde L., Hill R. S., Meyer S. S., Barnes C., Bennett C. L., Halpern M., Jarosik N., Kogut A., Komatsu E., Limon M., Page L., Tucker G. S., Weiland J. L., Wollack E., Wright E. L., First-Year Wilkinson Microwave Anisotropy Probe (WMAP) Observations: The Angular Power Spectrum, ApJS, 2003, vol. 148, p. 135

Hobson M. P., Efstathiou G. P., Lasenby A. N., General Relativity. Cambridge University Press Cambridge UK, 2006

Hobson M. P., Jaffe A. H., Liddle A. R., Mukeherjee P., Parkinson D., Bayesian Methods in Cosmology. Cambridge University Press Cambridge UK, 2010

Hook A., Baryogenesis from Hawking radiation, Phys. Rev. D, 2014, vol. 90, p. 083535

Hu W., Dodelson S., Cosmic Microwave Background Anisotropies, Ann. Rev. Astron. Astrophys., 2002, vol. 40, p. 171

Hubble E., A relation between distance and radial velocity among extra-galactic nebulae, Proc. Nat. Acad. Sci., 1929, vol. 15, p. 168

Humphreys L., Reid M., Moran J., Greenhill L., Argon A., Toward a New Geometric Distance to the Active Galaxy NGC 4258. III. Final Results and the Hubble Constant, ArXiv e-prints, 2013

Iocco, F. and Pato, M. and Bertone, G. Evidence for dark matter in the inner Milk Way, Nature Physics, 2015, pp 1-4

Ishak M., Richardson J., Garred D., Whittington D., Nwankwo A., Sussman R., Dark energy or apparent acceleration due to a relativistic cosmological model more complex than the Friedmann-Lemaitre-Robertson-Walker model?, Phys. Rev. D, 2008, vol. 78, p. 123531

Ishak M., Richardson J., Garred D., Whittington D., Nwankwo A., Sussman R., Erratum: Dark energy or apparent acceleration due to a relativistic cosmological model more complex than the Friedmann-Lemaitre-Robertson-Walker model? [Phys. Rev. D 78, 123531 (2008)], Phys. Rev. D, 2011, vol. 84, p. 089902 
Jacobson T., Thermodynamics of space-time: The Einstein equation of state, Phys.Rev.Lett., 1995, vol. 75, p. 1260

Järv L., Mohaupt T., Saueressig F., Phase space analysis of quintessence cosmologies with a double exponential potential, J. Cosmology Astropart. Phys., 2004, vol. 8, p. 16

Jesus J. F., Andrade-Oliveira, F. CCDM Model with Spatial Curvature and The Breaking of "Dark Degeneracy" (em prep.) , 2015

Jesus J. F., Oliveira, F. A. Basilakos S., Lima J. A. S., Newtonian perturbations on models with matter creation, Phys. Rev. D, 2011, vol. 84, p. 063511

Jesus J. F., Pereira S. H., CCDM model from quantum particle creation: constraints on dark matter mass, J. Cosmology Astropart. Phys., 2014, vol. 7, p. 40

Jesus J. F., Santos R. C., Alcaniz J. S., Lima J. A. S., New coupled quintessence cosmology, Phys. Rev. D, 2008, vol. 78, p. 063514

Kamenshchik A., Moschella U., Pasquier V., An alternative to quintessence, Physics Letters B, 2001, vol. 511, p. 265

Kass R. E., Raftery A. E., Bayes Factors, Journal of the American Statistical Association, 1995, vol. 90, p. 773

Kolb E. W., Turner M. S., The Early universe, Front. Phys., 1990, vol. 69, p. 1

Komatsu E., Dunkley J., Nolta et al. Five-Year Wilkinson Microwave Anisotropy Probe (WMAP) Observations: Cosmological Interpretation, ArXiv e-prints, 2008

Komatsu E., Smith K. M., Dunkley J., et al. Seven-Year Wilkinson Microwave Anisotropy Probe (WMAP) Observations: Cosmological Interpretation, ArXiv e-prints, 2010

Kowalski M., Rubin D., Aldering G., et al. Improved Cosmological Constraints from New, Old, and Combined Supernova Data Sets, ApJ, 2008, vol. 686, p. 749

Kragh H. S., Overduin J. M., The weight of the vacuum, 2014

Krauss L. M., Old Galaxies at High Redshift and the Cosmological Constant, ApJ, 1997, vol. 480 , p. 466 
Laganá T. F., Martinet N., Durret F., Lima Neto G. B., Maughan B., Zhang Y.-Y., A comprehensive picture of baryons in groups and clusters of galaxies, A\&A, 2013, vol. 555, p. A66

Landau L. D., Lifshitz E. M., The classical theory of fields. Course of theoretical physics Pergamon International Library of Science, Technology, Engineering and Social Studies, Oxford: Pergamon Press, 3rd rev. engl. edition, 1971

Lemaître G., Un Univers homogène de masse constante et de rayon croissant rendant compte de la vitesse radiale des nébuleuses extra-galactiques, Annales de la Societé Scientifique de Bruxelles, 1927, vol. 47, p. 49

Lemaitre G., Evolution of the Expanding Universe, Proceedings of the National Academy of Science, 1934, vol. 20, p. 12

Lemaître G. A., MacCallum M. A. H., The Expanding Universe, General Relativity and Gravitation, 1997, vol. 29, p. 641

Leon G., Saridakis E. N., Phantom dark energy with varying-mass dark matter particles: Acceleration and cosmic coincidence problem, Physics Letters B, 2010, vol. 693, p. 1

Lewis A., Bridle S., Cosmological parameters from CMB and other data: A Monte Carlo approach, Phys. Rev. D, 2002, vol. 66, p. 103511

Li M., Li X.-D., Wang S., Wang Y., Dark Energy, Communications in Theoretical Physics, 2011, vol. 56, p. 525

Liddle A. R., How many cosmological parameters?, MNRAS, 2004, vol. 351, p. L49

Lima J. A., Cunha J. V., Alcaniz J. S., Constraining the dark energy with galaxy cluster x-ray data, Phys. Rev. D, 2003a, vol. 68, p. 023510

Lima J. A., Cunha J. V., Alcaniz J. S., Constraining the dark energy with galaxy cluster x-ray data, Phys. Rev. D, 2003b, vol. 68, p. 023510

Lima J. A. S., Thermodynamics of decaying vacuum cosmologies, Phys. Rev. D, 1996, vol. 54 , p. 2571 
Lima J. A. S., Alternative Dark Energy Models: An Overview, Brazilian Journal of Physics, 2004, vol. 34, p. 194

Lima J. A. S., Alcaniz J. S., Dark Energy and the Angular Size-Redshift Diagram for Milliarcsecond Radio Sources, ApJ, 2002, vol. 566, p. 15

Lima J. A. S., Alcaniz J. S., Cunha J. V., A simplified approach for Chaplygin-type cosmologies, Astroparticle Physics, 2008

Lima J. A. S., Basilakos S., Costa F. E. M., New cosmic accelerating scenario without dark energy, Phys. Rev. D, 2012, vol. 86, p. 103534

Lima J. A. S., Basilakos S., Solà J., Expansion history with decaying vacuum: a complete cosmological scenario, MNRAS, 2013, vol. 431, p. 923

Lima J. A. S., Calvão M. O., Waga I., Cosmology, Thermodynamics and Matter Creation in: Frontier Physics, Essays in Honor of Jayme Tiomno. World Scientific, 1991

Lima J. A. S., Calvão M. O., Waga I., Cosmology, Thermodynamics and Matter Creation, ArXiv e-prints, 2007

Lima J. A. S., Cunha J. V., Alcaniz J. S., Simplified quartessence cosmology, Astroparticle Physics, 2009, vol. 31, p. 233

Lima J. A. S., Germano A. S. M., On the equivalence of bulk viscosity and matter creation, Physics Letters A, 1992, vol. 170, p. 373

Lima J. A. S., Germano A. S. M., Abramo L. R. W., FRW-type cosmologies with adiabatic matter creation, Phys. Rev. D, 1996, vol. 53, p. 4287

Lima J. A. S., Graef L. L., Pavón D., Basilakos S., Cosmic acceleration without dark energy: background tests and thermodynamic analysis, J. Cosmology Astropart. Phys., 2014, vol. 10, p. 42

Lima J. A. S., Jesus J. F., Oliveira, F. A. CDM accelerating cosmology as an alternative to $\Lambda$ CDM model, J. Cosmology Astropart. Phys., 2010, vol. 11, p. 27 
Lima J. A. S., Jesus J. F., Oliveira, F. A. Note on "Continuous matter creation and the acceleration of the universe: the growth of density fluctuations", General Relativity and Gravitation, 2011, vol. 43, p. 1883

Lima J. A. S., Maia J. M. F., Some Cosmological Consequences of a $\Lambda$-TERM Varying as $\beta H^{2}+\alpha R^{-n}$, Modern Physics Letters A, 1993, vol. 8, p. 591

Lima J. A. S., Maia J. M. F., Deflationary cosmology with decaying vacuum energy density, Phys. Rev. D, 1994, vol. 49, p. 5597

Lima J. A. S., Maia J. M. F., Pires N., Constraints from Big Bang Nucleosynthesis on a Time-Varying Cosmological Constant, 2000, vol. 198, p. 111

Lima J. A. S., Silva F. E., Santos R. C., Accelerating cold dark matter cosmology $\left(\Omega_{\Lambda} \equiv 0\right)$, Classical and Quantum Gravity, 2008

Lima J. A. S., Trodden M., Decaying vacuum energy and deflationary cosmology in open and closed universes, Phys. Rev. D, 1996, vol. 53, p. 4280

Lima J. A. S., Zanchin V., Brandenberger R., On the Newtonian cosmology equations with pressure, MNRAS, 1997, vol. 291, p. L1

Lin Y.-T., Mohr J. J., Stanford S. A., Near-Infrared Properties of Galaxy Clusters: Luminosity as a Binding Mass Predictor and the State of Cluster Baryons, ApJ, 2003, vol. 591, p. 749

Linder E. V., Exploring the Expansion History of the Universe, Physical Review Letters, 2003, vol. 90, p. 091301

Luzzi G., Shimon M., Lamagna L., Rephaeli Y., De Petris M., Conte A., De Gregori S., Battistelli E. S., Redshift Dependence of the Cosmic Microwave Background Temperature from Sunyaev-Zeldovich Measurements, ApJ, 2009, vol. 705, p. 1122

McDonald P., et al., The Linear theory power spectrum from the Lyman-alpha forest in the Sloan Digital Sky Survey, Astrophys.J., 2005, vol. 635, p. 761

McInnes B., What if $w<-1$ ?, 2002 
Maia J. M., Lima J. A., Scalar field description of decaying- $\Lambda$ cosmologies, Phys. Rev. D, 2002, vol. 65, p. 083513

Makler M., de Oliveira S. Q., Waga I., Constraints on the generalized Chaplygin gas from supernovae observations, Physics Letters B, 2003, vol. 555, p. 1

Martin J., Everything you always wanted to know about the cosmological constant problem (but were afraid to ask), Comptes Rendus Physique, 2012, vol. 13, p. 566

McCrea W. H., Relativity Theory and the Creation of Matter, Royal Society of London Proceedings Series A, 1951, vol. 206, p. 562

Mimoso J. P., Pavón D., Entropy evolution of universes with initial and final de Sitter eras, Phys. Rev. D, 2013, vol. 87, p. 047302

Moresco M., Cimatti A., Jimenez R., et al. Improved constraints on the expansion rate of the Universe up to $\mathrm{z} \sim 1.1$ from the spectroscopic evolution of cosmic chronometers, J. Cosmology Astropart. Phys., 2012, vol. 8, p. 6

Mukhanov V., Physical Foundations of Cosmology. Cambridge University Press Cambridge UK, 2005

Nayeri A., Padmanabhan T., A Possible Newtonian interpretation of relativistic cosmological perturbation theory, 1998

North J. D., The measure of the universe. A history of modern cosmology, Oxford: Clarendon Press, 1965, 1965

Overduin J. M., Cooperstock F. I., Evolution of the scale factor with a variable cosmological term, Phys. Rev. D, 1998, vol. 58, p. 043506

Özer M., Taha M. O., A possible solution to the main cosmological problems, Physics Letters B, 1986, vol. 171, p. 363

Pace F., Waizmann J.-C., Bartelmann M., Spherical collapse model in dark energy cosmologies, 2010 
Padmanabhan T., Choudhury T. R., A theoretician's analysis of the supernova data and the limitations in determining the nature of dark energy, Mon. Not. Roy. Astron. Soc., 2003, vol. 344 , p. 823

Parker L., Particle Creation in Expanding Universes, Phys. Rev. Lett., 1968, vol. 21, p. 562

Parker L., Particle creation and particle number in an expanding universe, Journal of Physics A Mathematical General, 2012, vol. 45, p. K4023

Parker L., Raval A., A New Look at the Accelerating Universe, Physical Review Letters, 2001, vol. 86 , p. 749

Parker L., Vanzella D. A., Acceleration of the universe, vacuum metamorphosis, and the large-time asymptotic form of the heat kernel, Phys. Rev. D, 2004, vol. 69, p. 104009

Pavon D., The generalised second law and extended thermodynamics, Classical and Quantum Gravity, 1990, vol. 7, p. 487

Pavón D., Nonequilibrium fluctuations in cosmic vacuum decay, Phys. Rev. D, 1991, vol. 43, p. 375

Pavón D., Radicella N., Does the entropy of the Universe tend to a maximum?, General Relativity and Gravitation, 2013, vol. 45, p. 63

Peacock J. A., Cosmological Physics, by John A. Peacock, pp. 704. ISBN 052141072X. Cambridge, UK: Cambridge University Press, January 1999.. Cambridge University Press, 1999

Peebles P. J., Ratra B., The cosmological constant and dark energy, Reviews of Modern Physics, 2003, vol. 75, p. 559

Peebles P. J. E., The large-scale structure of the universe, Research supported by the National Science Foundation. Princeton, N.J., Princeton University Press, 1980. 435 p.. Research supported by the National Science Foundation. Princeton University Press Princeton USA, 1980

Peebles P. J. E., Principles of Physical Cosmology. Princeton University Press Princeton, USA, 1993 
Peebles P. J. E., Seeing Cosmology Grow, ARA\&A, 2012, vol. 50, p. 1

Penzias A. A., Wilson R. W., A Measurement of Excess Antenna Temperature at 4080 Mc/s., ApJ, 1965, vol. 142, p. 419

Percival W. J., Reid B. A., Eisenstein D. J., et al. Baryon acoustic oscillations in the Sloan Digital Sky Survey Data Release 7 galaxy sample, MNRAS, 2010, vol. 401, p. 2148

Pereira S. H., Bessa C. H. G., Lima J. A. S., Quantized fields and gravitational particle creation in $\mathrm{f}(\mathrm{R})$ expanding universes, ArXiv e-prints, 2009

Perico E. L. D., Lima J. A. S., Basilakos S., Solà J., Complete cosmic history with a dynamical $\Lambda=\Lambda(\mathrm{H})$ term, Phys. Rev. D, 2013, vol. 88, p. 063531

Perlmutter S., Turner M. S., White M. J., Constraining dark energy with SNe Ia and large-scale structure, Phys. Rev. Lett., 1999, vol. 83, p. 670

Pires N., Zhu Z.-H., Alcaniz J. S., Lookback time as a test for brane cosmology, Phys. Rev. D, 2006, vol. 73, p. 123530

Planck Collaboration XVI: Ade, P. A. R. Aghanim N., Armitage-Caplan C., Arnaud M., Ashdown M., Atrio-Barandela F., Aumont J., Baccigalupi C., Banday A. J., et al. Planck 2013 results. XVI. Cosmological parameters, ArXiv e-prints, 2013

Polchinski J., The Cosmological Constant and the String Landscape, 2006, pp 216-236

Press W. H., Teukolsky S. A., Vetterling W. T., Flannery B. P., Numerical recipes in FORTRAN. The art of scientific computing, 2nd ed.. Cambridge: University Press Cambridge UK, 1992

Prigogine I., Geheniau J., Gunzig E., Nardone P., Thermodynamics and cosmology, General Relativity and Gravitation, 1989, vol. 21, p. 767

Radicella N., Pavón D., A thermodynamic motivation for dark energy, General Relativity and Gravitation, 2012, vol. 44, p. 685

Ramos R. O., Vargas dos Santos M., Waga I., Matter creation and cosmic acceleration, Phys. Rev. D, 2014, vol. 89, p. 083524 
Randall L., Sundrum R., An Alternative to Compactification, Physical Review Letters, 1999a, vol. 83, p. 4690

Randall L., Sundrum R., Large Mass Hierarchy from a Small Extra Dimension, Physical Review Letters, 1999b, vol. 83, p. 3370

Rapetti D., Allen S. W., Mantz A., The prospects for constraining dark energy with future X-ray cluster gas mass fraction measurements, MNRAS, 2008, vol. 388, p. 1265

Reis R. R., Domain of validity of the evolution of perturbations in Newtonian cosmology with pressure, Phys. Rev. D, 2003a, vol. 67, p. 087301

Reis R. R., Erratum: Domain of validity of the evolution of perturbations in Newtonian cosmology with pressure [Phys. Rev. D 67, 087301 (2003)], Phys. Rev. D, 2003b, vol. 68, p. 089901

Riess A. G., Filippenko A. V., Challis P., et al. Observational Evidence from Supernovae for an Accelerating Universe and a Cosmological Constant, AJ, 1998, vol. 116, p. 1009

Robertson H. P., Kinematics and World-Structure II., ApJ, 1936, vol. 83, p. 187

Roos M., Dark Matter: The evidence from astronomy, astrophysics and cosmology, ArXiv e-prints, 2010

Ross N. P., da Angela J., Shanks T., Wake D. A., Cannon R. D., et al., The 2dF-SDSS LRG and QSO Survey: The 2-Point Correlation Function and Redshift-Space Distortions, Mon.Not.Roy.Astron.Soc., 2007, vol. 381, p. 573

Rubin V. C., Ford Jr. W. K., Rotation of the Andromeda Nebula from a Spectroscopic Survey of Emission Regions, Astrophys. J., 1970, vol. 159, p. 379

Sahni V., Shtanov Y., Braneworld models of dark energy, Journal of Cosmology and AstroParticle Physics, 2003, vol. 11, p. 14

Salvatelli V., Said N., Bruni M., Melchiorri A., Wands D., Indications of a Late-Time Interaction in the Dark Sector, Phys. Rev. Lett., 2014, vol. 113, p. 181301

Santos J., Alcaniz J. S., Rebouças M. J., Carvalho F. C., Energy conditions in f(R) gravity, Phys. Rev. D, 2007, vol. 76, p. 083513 
Schwarz G., Estimating the Dimension of a Model, The Annals of Statistics, 1978, vol. 6, p. 461

Sen A. A., Sethi S., Quintessence model with double exponential potential, Physics Letters B, 2002, vol. 532, p. 159

Shapiro I. L., Solà J., The scaling evolution of the cosmological constant, Journal of High Energy Physics, 2002, vol. 2, p. 6

Shapiro I. L., Solà J., On the possible running of the cosmological "constant", Physics Letters B, 2009, vol. 682, p. 105

Shapiro I. L., Solà J., Stefancic H., Running $G$ and $\Lambda$ at low energies from physics at $M_{X}$ : possible cosmological and astrophysical implications, J. Cosmology Astropart. Phys., 2005, vol. 1, p. 12

Simon J., Verde L., Jimenez R., Constraints on the redshift dependence of the dark energy potential, Phys. Rev. D, 2005, vol. 71, p. 123001

Smith S., The Mass of the Virgo Cluster, ApJ, 1936, vol. 83, p. 23

Smoot G. F., et al., Structure in the COBE differential microwave radiometer first year maps, Astrophys. J., 1992, vol. 396, p. L1

Soares D., Einsteinian blunders, ArXiv Physics e-prints, 2005

Solà J., Cosmologies with a time dependent vacuum, Journal of Physics Conference Series, 2011, vol. 283, p. 012033

Sollerman J., Mörtsell E., Davis T. M., et al. First-Year Sloan Digital Sky Survey-II (SDSSII) Supernova Results: Constraints on Nonstandard Cosmological Models, ApJ, 2009, vol. 703 , p. 1374

Sorge F., Casimir effect in a weak gravitational field, Classical and Quantum Gravity, 2005, vol. 22 , p. 5109

Steigman G., Neutrinos And Big Bang Nucleosynthesis, ArXiv e-prints, 2012

Steigman G., Santos R. C., Lima J. A. S., An accelerating cosmology without dark energy, Journal of Cosmology and Astro-Particle Physics, 2009, vol. 6, p. 33 
Stern D., Jimenez R., Verde L., et al. Cosmic chronometers: constraining the equation of state of dark energy. I: H(z) measurements, J. Cosmology Astropart. Phys., 2010, vol. 2, p. 8

Sun M., Voit G. M., Donahue M., Jones C., Forman W., Vikhlinin A., Chandra Studies of the X-Ray Gas Properties of Galaxy Groups, ApJ, 2009, vol. 693, p. 1142

Suzuki N., Rubin D., Lidman C., et al. The Hubble Space Telescope Cluster Supernova Survey. V. Improving the Dark-energy Constraints above $z>1$ and Building an Earlytype-hosted Supernova Sample, ApJ, 2012, vol. 746, p. 85

Tegmark M., Blanton M. R., Strauss M. A., Hoyle F., et al. The Three-Dimensional Power Spectrum of Galaxies from the Sloan Digital Sky Survey, ApJ, 2004, vol. 606, p. 702

Tegmark M., et al., Cosmological Constraints from the SDSS Luminous Red Galaxies, Phys.Rev., 2006, vol. D74, p. 123507

Trotta R., Bayes in the sky: Bayesian inference and model selection in cosmology, Contemporary Physics, 2008, vol. 49, p. 71

Tryon E. P., Is the Universe a Vacuum Fluctuation?, Nature, 1973, vol. 246, p. 396

Tucker W., Blanco P., Rappoport S., David L., Fabricant D., Falco E. E., Forman W., Dressler A., Ramella M., 1E 0657-56: A Contender for the Hottest Known Cluster of Galaxies, ApJ, 1998, vol. 496, p. L5+

Turyshev S. G., Experimental Tests of General Relativity, Ann. Rev. Nucl. Part. Sci., 2008, vol. 58, p. 207

Vandenberg D. A., Bolte M., Stetson P. B., The Age of the Galactic Globular Cluster System, ARA\&A, 1996, vol. 34, p. 461

Vargas dos Santos M., Waga I., Ramos R. O., Degeneracy between CCDM and $\$ \backslash$ Lambda $\$$ CDM cosmologies, ArXiv e-prints, 2014

Viel M., Haehnelt M. G., Springel V., Inferring the dark matter power spectrum from the Lyman-alpha forest in high-resolution QSO absorption spectra, Mon.Not.Roy.Astron.Soc., 2004, vol. 354, p. 684 
Vikhlinin A., et al., X-ray Cluster Cosmology, 2009

Waga I., Decaying vacuum flat cosmological models - Expressions for some observable quantities and their properties, apj, 1993, vol. 414, p. 436

Walker A. G., On Milne's theory of world structure, Proc. Lond. Math. Soc., 1936, vol. 42, p. 90

Wang P., Meng X.-H., Can vacuum decay in our universe?, Classical and Quantum Gravity, 2005, vol. 22 , p. 283

Wang Y., Wands D., Xu L., De-Santiago J., Hojjati A., Cosmological constraints on a decomposed Chaplygin gas, Phys. Rev. D, 2013, vol. 87, p. 083503

Weinberg S., Gravitation and Cosmology: Principles and Applications of the General Theory of Relativity. John Wiley \& Sons, pp. 688, 1972

Weinberg S., The cosmological constant problem, Reviews of Modern Physics, 1989, vol. 61

Weinberg S., Cosmology. Oxford University Press, 2008

White M., et al. Dark Matter From Cosmological Probes, http://www.cosmos.esa.int/documents/387566/387653/Ferrara_Dec5_09h00_White _DMCosmology.pdf, 2014

White S. D. M., Rees M. J., Core condensation in heavy halos - A two-stage theory for galaxy formation and clustering, MNRAS, 1978, vol. 183, p. 341

White S. M., Navarro J. F., Evrard A. E., Frenk C. S., The baryon content of galaxy clusters: a challenge to cosmological orthodoxy, Nature, 1993, vol. 366, p. 429

Zel'dovich Y. B., The Cosmological constant and the theory of elementary particles, Sov. Phys. Usp., 1968, vol. 11, p. 381

Zeldovich Y. B., The Birth of a Closed Universe and the Anthropogenic Principle, Soviet Astronomy Letters, 1981, vol. 7, p. 322

Zhang C., Zhang H., Yuan S., et al. Four new observational H(z) data from luminous red galaxies in the Sloan Digital Sky Survey data release seven, Research in Astronomy and Astrophysics, 2014, vol. 14, p. 1221 
Zimdahl W., Fabris J. C., Chaplygin gas with non-adiabatic pressure perturbations, Classical and Quantum Gravity, 2005, vol. 22, p. 4311

Zimdahl W., Pavón D., Cosmology with adiabatic matter creation, Physics Letters A, 1993, vol. 176 , p. 57

Zimdahl W., Schwarz D. J., Balakin A. B., Pavón D., Cosmic antifriction and accelerated expansion, Phys. Rev. D, 2001, vol. 64, p. 063501

Zwicky F., Die Rotverschiebung von extragalaktischen Nebeln, Helvetica Physica Acta, 1933, vol. 6, p. 110 
Apêndice 

Apêndice A

\section{Análise Estatística}

Parte fundamental da Cosmologia contemporânea está na comparação e nos testes de modelos de universo. O grande número de dados observacionais exige a adoção de uma metodologia estatística adequada. Além disso, a cosmologia observacional deve lidar com o chamado problema inverso, i.e., dado um conjunto de dados é necessário converter as medidas em informação objetiva.

A análise Bayesiana fornece métodos de estimativas de parâmetros, seleção de modelos e testes de hipóteses, sendo uma das principais ferramentas em cosmologia. Discussões aprofundadas das técnicas estatísticas podem ser encontradas em Press et al. (1992) e, com enfoque em cosmologia, em Liddle (2004): Trotta (2008): Heavens (2009): Hobson et al. (2010) entre outros.

Nesta tese, utilizamos por repetidas vezes o teste estatístico de $\chi^{2}$. Suponhamos que estejamos ajustando $\mathrm{N}$ medidas $\left(x_{i}, y_{i}\right)$, com $i=1, \ldots, N$ a um modelo que tenha um conjunto de $M$ parâmetros livres $\mathbf{s}=\left(q_{1}, q_{2}, \ldots, q_{M}\right)$. Tal modelo prevê uma relação funcional entre as medidas e as variáveis:

$$
y(x)=y(x \mid \mathbf{s})
$$

onde a dependência nos parâmetros é indicada na função do lado direito. O problema a ser resolvido é o de encontrar os valores dos parâmetros livres $q_{i}$ que melhor ajustam o modelo $y(x)$ ao conjunto de $\mathrm{N}$ dados $\left(x_{i}, y_{i}\right)$. Nesse contexto, utilizaremos os estimadores de máxima verossimilhança (Press et al., 1992).

Vamos supor que cada dado $y_{i}$ possua um erro de medida independentemente aleatório e distribuído normalmente em torno do modelo "real" $y(x)$, com desvio padrão $\sigma_{i}$. A probabilidade do conjunto de dados é o produto das probabilidades de cada ponto: 


\begin{tabular}{|c|cccccc|}
\hline \hline & \multicolumn{6}{|c|}{$M$} \\
$\mathrm{p}$ & 1 & 2 & 3 & 4 & 5 & 6 \\
\hline \hline $68.3 \%$ & 1.00 & 2.30 & 3.53 & 4.72 & 5.89 & 7.04 \\
\hline $95.4 \%$ & 4.00 & 6.17 & 8.02 & 9.70 & 11.3 & 12.8 \\
\hline $99.73 \%$ & 9.00 & 11.8 & 14.2 & 16.3 & 18.2 & 20.1 \\
\hline $99.99 \%$ & 15.1 & 18.4 & 21.1 & 23.5 & 25.7 & 27.8 \\
\hline
\end{tabular}

Tabela A.1 - Valor do $\Delta \chi^{2}$ em função do nível de confiança e do número de parâmetros livres $M$. Tabela extraída de (Press et al., 1992).

$$
P \propto \prod_{i=1}^{N}\left\{\exp \left[-\frac{1}{2}\left(\frac{y_{i}-y\left(x_{i} \mid \mathbf{s}\right)}{\sigma_{i}}\right)^{2}\right] \Delta y\right\}
$$

onde o termo $\Delta y$ foi incluído para contabilizar uma pequena variação em torno dos valores possíveis de $y_{i}$. A probabilidade máxima na expressão (A.2) é equivalente ao valor mínimo da expressão:

$$
\frac{1}{2} \sum_{i=1}^{N} \frac{\left[y_{i}-y\left(x_{i} \mid \mathbf{s}\right)\right]^{2}}{\sigma_{i}^{2}}-N \log \Delta y .
$$

Neste ponto, é útil definirmos a função $\chi^{2}$ :

$$
\chi^{2} \equiv \sum_{i=1}^{N} \frac{\left[y_{i}-y\left(x_{i} \mid \mathbf{s}\right)\right]^{2}}{\sigma_{i}^{2}} .
$$

Em termos da verossimilhança, a expressão (A.4) pode ser escrita como $\chi^{2}=-2 \ln (A \mathcal{L})$, onde $A$ é uma constante e $\mathcal{L}$ é a verossimilhança. Como $N$ e $\Delta y$ não dependem dos parâmetros s, maximizar a probabilidade (A.2) é equivalente a minimizar o valor do $\chi^{2}$.

Um método particularmente simples de encontrar o conjunto de parâmetros de melhor ajuste é através da busca em uma grade. Nesse método, toma-se pontos igualmente distanciados dentro de uma região espaço de parâmetros em que se espera que o mínimo esteja e dentro dos limites físicos dos parâmetros. Em cada ponto é calculado o valor do $\chi^{2}$ e então se busca pelo ponto em que o $\chi^{2}$ é mínimo. Este método possui a vantagem em relação a outros, uma vez que o mínimo global pode não pertencer ao intervalo físico e a grade gerada pode ser aproveitada na estimativa de erros. Entretanto, o método só é computacionalmente viável quando o modelo possui poucos parâmetros a ser ajustados $(M \leq 3)$. 
As estimativas de erros dos parâmetros do modelo ajustado podem ser realizadas utilizando os níveis de $\chi^{2}$, como uma boa aproximação. As chamadas regiões ou intervalos de confiança no espaço de parâmetros correspondem a uma certa fração da distribuição total. Pode-se mostrar que essas regiões podem ser obtidas através das curvas de nível de $\chi^{2}$. Portanto, cada nível $\Delta \chi^{2}=\chi^{2}-\chi_{\min }^{2}$ corresponde à probabilidade dos parâmetros estarem dentro da região delimitada pelo dado nível (Press et al., 1992). Alguns valores do $\Delta \chi^{2}$ em função dos níveis de confiança estatística são mostrados na tabela A.1.

Da inferência Bayesiana, obtemos o conceito de marginalização sobre um parâmetro adicional (nuisance parameter) (Gregory, 2005). Suponhamos que temos probabilidade $p\left(q_{1}, q_{2} \mid D\right)$ de dois parâmetros livres do modelo, $q_{1}$ e $q_{2}$, e um conjunto de medidas $D$. Se, por uma acaso, não estamos interessados no comportamento de um dos parâmetros, a estatística Bayesiana fornece um meio de inferir a probabilidade dependente apenas do parâmetro desejado. Como ilustração, no caso em que desejamos eliminar a dependência da densidade de probabilidade com o parâmetro adicional $q_{2}$, temos:

$$
p\left(q_{1}\right)=\int p\left(q_{1}, q_{2} \mid D\right) d q_{2}
$$

onde o resultado pode ser estendido para 3 ou mais variáveis.

Outro problema recorrente é a escolha de modelos. Um bom modelo físico deve ser capaz não apenas de ajustar bem as medidas, mas fazê-lo da forma mais econômica possível. Por exemplo, em um ajuste por regressão polinomial, o princípio da máxima verossimilhança tenderá a escolher a opção de maior dimensão possível. Critérios de informação têm como objetivo contornar esse tipo de problema de forma objetiva, de acordo com o princípio de parcimônia estatística.

\begin{tabular}{cl}
\hline \hline$\Delta$ BIC & Evidência Contra um Modelo \\
\hline $0-2$ & Desconsiderável \\
$2-6$ & Positiva \\
$6-10$ & Forte \\
$>10$ & Muito Forte \\
\hline
\end{tabular}

Tabela A.2 - Critérios quantitativos para a seleção de modelos via BIC (Kass e Raftery, 1995).

Dentro do formalismo de Bayes, a comparação entre dois modelos pode ser realizada através do critério de inferência Bayesiana (BIC), definido como (Schwarz, 1978; Liddle, 
2004; Hobson et al., 2010)

$$
B I C=-2 \ln \left(\mathcal{L}_{\max }\right)+M \ln (N)
$$

onde $\mathcal{L}_{\max }$ é o valor máximo da verossimilhança em dado modelo, $M$ é o número de parâmetros livres do modelo e $N$ é o número de dados observacionais. Um dado modelo $\mathcal{A}$ é mais favorecido quando o seu BIC é o menor possível. Assim, um método de comparar modelos é através da grandeza $\Delta B I C=B I C_{\mathcal{A}}-B I C_{\mathcal{B}}$. Em Kass e Raftery (1995), são fornecidos critérios quantitativos na seleção de modelos, baseados no fator de Bayes (Tabela A.2). 